\title{
The fourth spectrum of iridium (Ir IV)
}

\author{
Vladimir I. Azarov ${ }^{1}$ and Robert R. Gayasov ${ }^{2}$ \\ ${ }^{1}$ Department of Research and Development, Parametric Technology Corporation, \\ 140 Kendrick Street, Needham, MA 02494, USA. E-mail: vlad_azarov@yahoo.com \\ ${ }^{2}$ Institute of Spectroscopy, Russian Academy of Sciences, \\ Troitsk, Moscow region, Russian Federation 142092.
}

\begin{abstract}
The spectrum of three times ionized iridium, Ir IV, was investigated in the 650 - $2045 \AA$ wavelength region. The analysis has led to the determination of the $5 \mathrm{~d}^{6}, 5 \mathrm{~d}^{5} 6 \mathrm{~s}$ and $5 \mathrm{~d}^{5} 6 \mathrm{p}$ configurations. Twenty-nine of 34 theoretically possible $5 d^{6}$ levels, 44 of 74 possible $5 d^{5} 6$ s levels and 150 of 214 possible $5 d^{5} 6 p$ levels have been established. The levels are based on 1348 classified spectral lines. The level structure and transition probabilities were calculated using the orthogonal operators technique. The energy parameters have been determined by the least squares fit to the observed levels. Calculated energy values and $L S$-compositions obtained from the fitted parameter values are given. The level optimization procedure and the determination of uncertainties of the obtained energy level values are discussed.
\end{abstract}

PACS Ref: 32.30.-r, 32.30.Jc

Keywords: spectral line identification, energy levels, atomic spectra, vacuum ultraviolet, three times ionized iridium, $\mathrm{Ir}^{3+}$.

\section{Contents}

1. Introduction and experiment 1

2. Level optimization $\quad 2$

3. Analysis $\quad 5$

4. Calculations $\quad 7$

$\begin{array}{ll}\text { References } & 8\end{array}$

Explanation of Tables $\quad 9$

Tables

1. Classified lines in the $\left(5 d^{6}+5 d^{5} 6 s\right)-5 d^{5} 6 p$ transition array of Ir IV.

2. Experimental and calculated energy levels $\left(\mathrm{cm}^{-1}\right)$ in the $5 \mathrm{~d}^{6}$ and $5 \mathrm{~d}^{5} 6 \mathrm{~s}$ configurations of Ir IV.

3. Experimental and calculated energy levels $\left(\mathrm{cm}^{-1}\right)$ in the $5 d^{5} 6 p$ and $5 d^{4} 6 s 6 p$ configurations of Ir IV.

4. Fitted and calculated parameter values $\left(\mathrm{cm}^{-1}\right)$ in the $5 d^{6}, 5 d^{5} 6 \mathrm{~s}$ and $5 d^{4} 6 s^{2}$ configurations of Ir IV.

5. Fitted and calculated parameter values $\left(\mathrm{cm}^{-1}\right)$ in the $5 d^{5} 6 p, 5 d^{4} 6 s 6 p$ and $5 d^{3} 6 s^{2} 6 p$ configurations of Ir IV. 


\section{Introduction and experiment}

A 100-year period of investigation of iridium ions having rather simple ground-state configurations $5 \mathrm{~d}$, $5 d^{2}, 5 d^{8}$, and $5 d^{9}$ was started by Kayser [1] in 1898 and was finished in 1995-1998 when the spectra of Ir VIII and Ir IX [2,3] had been studied. During this long-lasting period, many authors have contributed to analyses of the spectra Ir I, Ir II, Ir VIII, and Ir IX [4-9]. Analyses of the more complicated iridium spectra of the ions with three to five electrons in the outer 5d-shell, such as Ir VII, Ir VI, and Ir V [10-13], have become possible due to refinement in theoretical calculations utilizing the orthogonal operators approach [14-17], development of the program suite IDEN [18,19], and successful analyses of the neighboring Os VI, Os V, and Os IV spectra [2022].

The objective of the present work is to study the spectrum of three times ionized iridium, Ir IV, which has not been investigated previously. The ion has the $5 \mathrm{~d}^{6}$ ground-state configuration. Due to the overlapping of the lowest three configurations in both parities, the Ir IV spectrum is extremely complex.

In the most recent investigations of iridium spectra $[11,12]$ we have utilized two sets of spectrograms photographed in the wavelength region of $300-2100 \AA$ at the Spectroscopic Laboratory of St. Francis Xavier University, Antigonish, Canada, and the Institute of Spectroscopy, Troitsk, Russian Federation. Although both sets of spectrograms were suitable for the analysis of Ir IV, we used the Troitsk set in our study because it has more spectral lines of lower ionization stages, while the Antigonish set was helpful in the line discrimination on ionization stages. The experimental details of this set are given in the Ir VI paper [12]. At the Troitsk laboratory a $6.65-\mathrm{m}$ normal incidence spectrograph was used, which has the plate factor of $1.25 \AA / \mathrm{mm}$ in the first order. The sources were vacuum sliding and triggered sparks with operating voltage of $4.0-4.5 \mathrm{kV}$. The sparks were fed from a low-inductance $10 \mu \mathrm{F}$ capacitor. In both laboratories the plates were measured on automatic comparators controlled by the system for automatic processing of photo-spectrograms [23] and initially reduced by using impurity lines of $\mathrm{O}, \mathrm{C}, \mathrm{N}, \mathrm{Al}$ and $\mathrm{Si}$ [24] present in the spectrograms. The initial uncertainty of wavelength measurements was about $0.007 \AA$ and $0.010 \AA$ for the wavelengths below and higher than about $1000 \AA$, respectively. It increased to about $0.020 \AA$ locally for some sub-regions due to the lack of standards, imperfection of the plate holder and deformation of photo-emulsion, most notably at the ends of the photographic plates. By using additional internally derived Ritz standards, as well as wavelengths measured in the second order of diffraction, we have improved the accuracy of our wavelength measurements in the course of the analysis and have the final standard deviation of isolated lines from the calculated positions within $0.005 \AA$ in the region of 650 - $2045 \AA$.

\section{Level optimization}

In the IDEN computer program [18,19], with which we perform spectra analyses, to optimize the energy level system having levels "connected" by wavenumbers of the identified spectral lines, an iterative procedure of refining values of the energy levels is utilized. The "weighted mean" technique is used for this purpose. The weighted mean of $n$ sample data elements with measured values $x_{i}$ having weights $w_{i}$ is determined as

$$
\bar{x}=\frac{\sum_{i=1}^{n} w_{i} x_{i}}{\sum_{i=1}^{n} w_{i}} .
$$

The biased weighted estimator of the sample variance can be shown as

$\sigma^{2}=\frac{\sum_{i=1}^{n} w_{i}\left(x_{i}-\bar{x}\right)^{2}}{\sum_{i=1}^{n} w_{i}}$

The unbiased weighted estimator of the sample variance can be computed as follows

$s^{2}=A \cdot \frac{\sum_{i=1}^{n} w_{i}\left(x_{i}-\bar{x}\right)^{2}}{\sum_{i=1}^{n} w_{i}}$,

where $A$ is a correction coefficient making the biased variance estimator unbiased

$A=\frac{\left(\sum_{i=1}^{n} w_{i}\right)^{2}}{\left(\sum_{i=1}^{n} w_{i}\right)^{2}-\sum_{i=1}^{n} w_{i}^{2}}$.

When all weights are equal, $w_{i}=w_{0}$, the coefficient becomes: $A=n /(n-1)$. In general, $A \rightarrow 1$ when $n \rightarrow \infty$. The most critical cases that need special consideration are: a) $n=1$ and b) when there is only one sample with a 
high weight and all other weights are negligible. In the latter case $A$ becomes very large. These cases are considered in Section 3.

For the weighted mean of a set of data, for which each element $x_{i}$ comes with known (or estimated) variance ${\sigma_{i}}^{2}$, and one possible choice for the weights is given by

$w_{i}=\frac{1}{\sigma_{i}^{2}}$.

The weighted mean in this case is

$\bar{x}=\frac{\sum_{i=1}^{n}\left(x_{i} \sigma_{i}^{-2}\right)}{\sum_{i=1}^{n} \sigma_{i}^{-2}}$,

and the variance of the weighted mean (WMV) is

$\sigma_{\bar{x}}^{2}=\frac{1}{\sum_{i=1}^{n} \sigma_{i}^{-2}}$,

which reduces to ${\sigma_{\bar{x}}}^{2}=\sigma_{0}^{2} / n$, when all $\sigma_{i}=\sigma_{0}$.

If, instead of variances ${\sigma_{i}}^{2}$ of the data elements, estimated uncertainties $u_{i}$ are specified, variances of the data elements can be obtained as $\sigma_{i}{ }^{2}=u_{i}{ }^{2}$. The only requirement for the estimated uncertainties $u_{i}$ of the data elements is the following. Regardless how the uncertainty of a data element is obtained, it must be evaluated as a standard uncertainty (standard deviation). We have to note that all uncertainties given in this paper are on the level of one standard deviation.

Typically, experimental data contain some inaccuracy in estimation of the variance/uncertainty attributed to each data point. Often the data points are obtained (and their uncertainties are estimated) by using references in interpolation or calibration procedures. Resulting data depend on the availability of references, their uncertainties and other factors. Thus, the obtained uncertainty estimations of data points may be inaccurate and need further justification. Therefore, a correction to account for the real data distribution could be used to adjust the above expression for variance (7). In unbiased form it is as follows

$s_{\bar{x}}^{2}=A \cdot \frac{1}{\sum_{i=1}^{n} \sigma_{i}^{-2}} \cdot \frac{\sum_{i=1}^{n}\left(\sigma_{i}^{-2} \cdot\left(x_{i}-\bar{x}\right)^{2}\right)}{n}$.

If all data variances are equal, $\sigma_{i}=\sigma_{0}$, then the weighted mean variance reduces to the standard deviation of the mean (squared): $s_{\bar{x}}^{2}=s^{2} / n$, where

$s^{2}=\frac{\sum_{i=1}^{n}\left(x_{i}-\bar{x}\right)^{2}}{n-1}$

is a simple standard deviation (squared) of the data points used in the calculation of the mean value.

We have to stress here that, if the uncertainties of the data points are correctly estimated, the variance given by the equation (7) may be considered as the uncertainty (squared) of the weighted mean for the given data set. The equation (8), in fact, re-estimates the data uncertainties based on the real deviations of the data points from the weighted mean values and uses these values for the WMV estimation. The variance given by the equation (8) does not include the value $\sigma_{0}$ if all input data variances are equal (e.g., $\sigma_{0}{ }^{2}$ ), and this equation may give a significantly lower value than the equation (7), or even a zero value. This indicates that the number of samples is too small, or the specified data uncertainties are overestimated (too large). If the equation (8) gives much larger value than the equation (7), it may indicate that the number of samples is too small, or the specified estimated uncertainties of the data points are underestimated (too small), or the data points are incorrectly measured.

In the case of the energy level optimization procedure (LOP) adopted in IDEN, the variables $\bar{x}$ and $x_{i}$ should be substituted as follows: $\bar{x}=\bar{E}$, where $\bar{E}$ is the weighted mean value of the given energy level, $x_{i}=E_{i} \pm v_{i}$, where $E_{i}$ is the value of the energy level connected to the given level by the transition with the wavenumber $v_{i}$ having uncertainty $\sigma_{i}$. The sign " $+"$ is used if the given level $E$ is the upper level and "-" is used if $E$ is the lower level for the transition. Thus, in each iteration cycle, we calculate $\bar{E}$ for the level $E$ using levels $E_{i}$ directly connected to the level $E$ by transitions. When we estimate the variance/uncertainty of $\bar{E}$ using the equation (7) or (8), we neglect dependency of the level $E_{i}$ on all wavenumbers taking part in the averaging, except for $v_{i}$. This is an insignificant limitation for our energy level system studied here.

In the spectra identification and level determination problem, the most accurate pieces of information (data elements) are intervals between pairs of levels. One group of such accurate intervals contains pairs of 
levels directly connected by identified transitions. Another group contains pairs of close same-parity levels, where splitting of each such pair is determined by several couples of identified transitions from/to the connecting levels of opposite parity. The spectral lines in each such couple are close and therefore the systematic uncertainty of their measurements is absorbed in the connecting level, thus giving the value of the two-level splitting with lower uncertainty. Usually, any sub-system or combination of the mentioned data elements has derived values with larger uncertainties. For instance, the derived distance between two oppositeparity levels, which are not directly connected by a transition, may have rather large uncertainty compared to the uncertainties of all wavenumbers in the system.

In publications, uncertainties of levels found in analyses are usually given with respect to the ground level having assigned uncertainty equal to zero. In the case when the ground level is not well determined, all levels may be presented as having a large estimated uncertainty. A better choice is to select some welldetermined level as a "base" level, give its uncertainty with respect to the ground level and present the uncertainties of all other levels with respect to the selected one. This approach is discussed in details in the publication describing the program for energy level optimization, LOPT [25].

For the comparison (e.g., along iso-electronic sequences) of obtained in analyses level uncertainties, the uncertainties given with respect to the ground level or some "base" level may not be convenient. First, the uncertainties may be too large, especially if they are given with respect to the ground level. Second, for the comparison of level uncertainties in different ions of an iso-electronic sequence, the uncertainties should be recalculated using the same "base" level, which may not be determined yet in all ions of the considered sequence.

For the purpose of the quality check of the analysis, we consider the uncertainty of determination of each given level with respect to the levels directly connected to it by identified transitions. Such values are usually smaller than values given with respect to the ground state or a "base" level, and they do not depend on the selection of the "base" level. In addition, such way of level uncertainty estimations comes naturally from the "old style" analyses when one checks levels in an even-odd level table along rows (even) and columns (odd) and calculates root-mean-square (rms) deviations of the experimental wavenumbers from the calculated (Ritz) values. We always perform this kind of level uncertainty estimations during the IDEN program runs with visualization, interactively adjusting the level position, and analyse the quality of the resulting level identification and determination. Such approach for estimation of the level uncertainty could be described as the following. In the program LOPT we may declare each of the $n$ existing levels to be a "base". Then, in the output file, each level we will contain $n-1$ uncertainty estimations with respect to $n-1$ "bases". Now for each level we can consider all these $n-1$ uncertainties and take the smallest one, or use the equation (7) to combine them. This is equivalent to a level declaration as a "base", analysis of uncertainty values given by LOPT for all other levels with respect to the given "base", and adoption of the smallest one or combination all of them using the equation (7).

The result of a spectrum analysis consists of a set of classified spectral lines, which are to be used for determination of the energy level structure. Ideally, if all identifications are correct, each level is connected with all other levels by transitions having wavenumbers with correctly estimated and comparable uncertainties; the resulting optimized (weighted mean) energy values will be the variances calculated from the equation (8) close to the values calculated from the equation (7). The values of the first and third factors in the equation (8) will be close to unity in this case.

However, the number of level connections is limited not only by selection rules, but also by experimental conditions. Many allowed transitions may not be observed or identified. It often happens that some spectral lines are masked, blended, or their identifications are doubtful. These lines may increase the third factor in the equation (8) significantly. In most of the cases, these lines can be detected and excluded from the level optimization. We also exclude from the LOP all levels determined by a single transition. From the other side, by raising the number of excluded and/or not observed spectral lines, one increases the first factor in the equation (8) and thus makes WMVs calculated by the equation (8) larger. It also makes the system less inter-connected and may even break it into unconnected fragments.

If the uncertainties of wavelengths of the spectral lines used in the optimization are estimated correctly, and all disturbed lines are removed from the optimization, we expect the values given for most levels by the expression (8) to be close to or smaller than those given by the expression (7). In our analyses we verify this relation for each level and use it as a quality check for the results of the LOP and the entire analysis. 


\section{Analysis}

The ground-state configuration of Ir IV is $5 \mathrm{~d}^{6}$ and the lowest two excited configurations of different parities are $5 \mathrm{~d}^{5} 6 \mathrm{~s}$ and $5 \mathrm{~d}^{5} 6 \mathrm{p}$. This ion belongs to the W I iso-electronic sequence. Only two members in the sequence have been investigated so far: W I and Re II [26-29]. In our publication of the Ir VI analysis [12], we described a procedure for estimation of parameters of Ir VI configurations through iso-electronic, iso-ionic and iso-nuclear comparison. It includes a record of all relevant known ions and references. Since then there no additional data on the neighboring ions have been published. These described data and extrapolation procedure are also suitable for obtaining initial parameters for the $\mathrm{Ir}^{3+}$ ion studied here. It should be noticed that a general procedure for inter- and extrapolation of energy parameters of ions in a larger (than a sequence) group of ions of neighboring elements (e.g., ions with open 5d-shell of the platinum group elements) was presented in Ref. [30]. This procedure is also designed for critical analyses of known experimental energy levels and parameters, for analyses of regularities in experimental parameters and deviations of experimental levels from their calculated positions, thus allowing systematic investigation of small magnetic and electrostatic effects. Unfortunately, the data on the W I and Re II spectra, which are in the same iso-electronic sequence as Ir IV, are not applicable in the extrapolation of parameters of Ir IV. Many studies show [14,31,32], although there is quite regular evolution of the experimental parameters or scaling factors of their ab initio values along the isoelectronic sequence, when the ionization stage lowers, the trends of parameters become more irregular. For instance, the mentioned factors for electrostatic parameters fall down sharply when we approach the third and second spectra.

The most useful in the estimation of Ir IV parameters were the published data on the neighboring Ir V, Ir VI, Os VI, Os V, and Os IV spectra [13,12,20-22] and our unpublished data on the neighboring Os III, Pt V, Pt IV, and Ir III spectra, analyses of which are currently being finalized and will be published soon. In fact, in the publication [12] it was mentioned that we were simultaneously making analyses of Ir VI, Ir IV and Ir III spectra utilizing the same sets of spectrograms having spectra photographed under the different source conditions. This allowed us to avoid line misidentifications in the very dense iridium spectra recorded, where arrays of spectral lines belonging to different ions are overlapping and it is difficult to distinguish lines belonging to ions of close ionization stages.

The process of finding levels, identification of lines and optimization of level energies (using the level optimization procedure, LOP) was carried out by utilizing the program suite IDEN [18,19] allowing visualization. The program LOPT [25] was used for verification of the optimized by IDEN energy values, and calculation of level and Ritz wavelengths uncertainties.

In the region of $650-2045 \AA$, we have identified 1348 spectral lines belonging to the $\left(5 d^{6}+5 d^{5} 6 s\right)$ $5 d^{5} 6 p$ transition array of Ir IV. Twenty-two spectral lines are doubly classified and two lines have triple classification. The list of identified lines is presented in Table 1. Transition probabilities $(g A)$ have been calculated from the final fitted parameter values using the orthogonal operators approach. Intensities of the spectral lines (Int) have been obtained by measuring the plate darkening and converting optical density to spectral intensity, and subtracting the spectral background approximated by a spline curve. The agreement between $g A$ and $I n t$ for the isolated unperturbed and unsaturated lines is quite good when we compare lines originating from the same upper level or from close upper levels. Taking into account the Boltzmann factor, describing a decrease of population for levels with an increasing energy, agreement between the presented $g \mathrm{~A}$ and Int values is good throughout the entire list. In each case when the intensity of an identified spectral line is notably higher than expected, we give the expected value in Table 1. The uncertainties of the Ritz wavelengths (Unc $\lambda_{\text {calc }}$ ) were calculated using the program LOPT [25]. The numbers $N_{\text {odd }}$ and $N_{\text {even }}$ of the observed transitions define the odd and even level involved in the particular transition and the difference between the experimental and Ritz wavelengths of the transition, $\Delta(\lambda)$, and they are helpful for detecting local systematic deviations (LSD, see Ref. [33]). Indeed, after the values of energy levels are optimized, one would expect the values of $\Delta(\lambda)$ to be randomly distributed in the linelist. For several reasons, such as ends of plates, defects in the plate holder, or lack of standards of wavelengths, $\Delta(\lambda)$ may exhibit the LSD effect which should only be considered for transitions having both levels derived from many significantly distant lines. Levels defined by a single or close multiple lines always result in $\Delta(\lambda)$ values with no systematic deviation, as the latter is absorbed in the level value. Therefore, such levels should not be accounted for in the LSD detection. Due to strong mixing of levels, labels in the $L S$-coupling scheme are omitted in Table 1. For each tabulated transition we also provide the following contents: a name of the linelist part ( $\mathrm{pa}-\mathrm{pg}$ ) to refer to the wavelength uncertainty of the 
given part as in Table A, the symbol "*" for transitions that were not used in the LOP, expected line intensity where appropriate, derived from the $g A$ and Int comparison, and comments for masked and blended lines, and lines that are also identified as belonging to another ion or impurity. We have excluded from LOP runs transitions corresponding to lines that are masked, much stronger than expected, have suspiciously large deviations, or have small $g A$ values for which chances of wrong identification are substantial. If the line is blended by a known line of the other ion or impurity, we specify the blending spectrum in parentheses following the "bl" symbol. If the line is also identified with a transition in another ion but does not appear as a blend in the spectrogram, we do not give the "bl" symbol, although blending is possible due to a near coincidence of wavelengths. In addition, if the line is also identified as another Ir ion (e.g., Ir III) and, when using the same linelist, the expected intensity of that identification (e.g., 400) is lower than the observed line intensity, we give "Ir III (400)".

For comparison of the $g A$ and Int values accounting for the Boltzmann factor, $\exp (-E / k T)$, we have used two sets of plates, which may have been obtained under somewhat different experimental conditions (and thus different $k T$ values). Linelist parts pa and pb - pg correspond to two different sets (exposures); parts pb $\mathrm{pd}$ and pe - pg correspond to two plates from the same set (exposure).

Experimental and calculated energy values of the even parity levels in the $5 \mathrm{~d}^{6}$ and $5 \mathrm{~d}^{5} 6$ s configurations and the odd parity levels in the $5 d^{5} 6 p$ and $5 d^{4} 6 s 6 p$ configurations of Ir IV are given in Table 2 and Table 3 , respectively. The uncertainties of the levels were calculated by the program LOPT [25] and given with respect to the "base" even level with $J=4$ at $71191.62 \mathrm{~cm}^{-1}$. A reasonable estimate of uncertainty of excitation energy for any level can be deduced from these data. As it was mentioned, we excluded from the LOP all levels determined by a single transition $\left(N_{\text {opt }}=1\right)$. For each such level, we set its value equal to the sum (if the level is odd) or the difference (if the level is even) of the opposite parity level value and the connecting wavenumber.

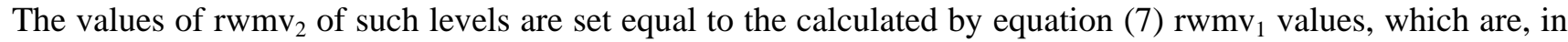
turn, equal to the estimated uncertainties of the corresponding wavenumbers (see, for instance, the level at $\left.152556.11 \mathrm{~cm}^{-1}, J=8\right)$.

In the present analysis, 29 of 34 theoretically possible $5 \mathrm{~d}^{6}$ levels, 44 of 74 possible $5 \mathrm{~d}^{5} 6 \mathrm{~s}$ levels and 150 of 214 possible $5 d^{5} 6 p$ levels have been established. Levels that have not been established either result in too weak transitions to be observed on our plates or are expected to give rise to only one or two relatively weak lines that cannot be distinguished without ambiguity.

From the beginning of the analysis, we have divided the linelist into seven parts, pa - pg (see Table 1, last column), and assigned to them initial estimated uncertainties. The assigned uncertainties are based on the available standards, distribution of the standards around the wavelength interpolation curve, and the expected increased uncertainty near ends of the plates. The root-mean-square (rms) deviation of wavelengths of the undisturbed experimental lines from their calculated (Ritz) positions were deduced after LSD corrections of the linelist in the course of the analysis and after the final LOP run, for each part. The linelist parts are presented in Table A below; each linelist part includes the number of lines used in the LOP.

\section{Table A}

Description of the linelist parts.

\begin{tabular}{llllllll}
\hline Linelist part & $\mathrm{pa}$ & $\mathrm{pb}$ & $\mathrm{pc}$ & $\mathrm{pd}$ & $\mathrm{pe}$ & $\mathrm{pf}$ & $\mathrm{pg}$ \\
Initial estimated uncertainty, $\mathrm{m} \AA$ & 10 & 10 & 15 & 20 & 15 & 10 & 15 \\
Resulting $r m s, \mathrm{~m} \AA$ & 4 & 4 & 5 & 6 & 5 & 3 & 6 \\
Final estimated uncertainty, $\mathrm{m} \AA$ & 5 & 5 & 7 & 10 & 7 & 5 & 7 \\
Number of lines used in LOP & 533 & 128 & 277 & 4 & 68 & 94 & 3
\end{tabular}

The rms deviation of all undisturbed identified lines from their Ritz positions, including lines not used in the LOP, is about $0.005 \AA$. Thus, our initial linelist has been significantly improved. The final estimated uncertainties of wavelength measurements that were used for the $\mathrm{rwmv}_{1}$ and $\mathrm{rwmv}_{2}$ calculations and in the program LOPT [25] are shown in Table A. These uncertainties are square root values of combinations of squares of the statistical and systematic uncertainties, where the latter were estimated from standard deviations of wavelengths of reference lines from their positions derived from the calibration curves. We have found that the optimized level values given by the IDEN's LOP and those given by the program LOPT are in a very good 
agreement: $95 \%$ of levels have differences within $0.03 \mathrm{~cm}^{-1}$, which is close to the rounding errors of wavelengths. The remaining $5 \%$ of the levels differ by less than $0.06 \mathrm{~cm}^{-1}$, which is a small fraction of their calculated uncertainties. The rounding error of the presented wavelengths is $<0.5 \mathrm{~m} \AA$. It corresponds to the wavenumber errors of $<0.01 \mathrm{~cm}^{-1}$ and $<0.11 \mathrm{~cm}^{-1}$ for the last and first lines presented in Table 1, respectively.

Comparison of the values of $\mathrm{rwmv}_{1}$ and $\mathrm{rwmv}_{2}$ in Tables 2 and 3 shows consistency: $\mathrm{rwmv}_{1} \geq \mathrm{rwmv}_{2}$ for most of the levels (83\%). Only 17 of 223 found levels $(8 \%)$ have rwmv 2 values $20 \%$ greater than rwmv values, and only two levels at $154852.06 \mathrm{~cm}^{-1}$ and $159629.42 \mathrm{~cm}^{-1}$ have the difference greater than $50 \%$ but less than $77 \%$. Both levels were represented in the LOP run by three transitions with relatively large but not critical deviations from the calculated values. These former and latter levels have nine and three transitions beyond the LOP supporting identified transitions, respectively. Therefore, the identification of these two levels is correct. We do not have cases when the value of the coefficient $A$ given by the equation (4) is large enough to treat the corresponding level in a special way, as we treated the levels determined by a single transition. The maximum value of 4.7 belongs to the level at $150748.58 \mathrm{~cm}^{-1}, J=1$. This level is determined by two transitions with consistent and relatively small $\mathrm{rwmv}_{1}$ and $\mathrm{rwmv}_{2}$.

The comparison of the shown in Tables 2 and 3 values $N, N_{o p t}, \mathrm{rwmv}_{1}$, and $\mathrm{rwmv}_{2}$ indicates the most accurately determined level at $71191.62 \mathrm{~cm}^{-1}$. It has the smallest $\operatorname{rwmv}_{1}\left(0.05 \mathrm{~cm}^{-1}\right), \operatorname{rwmv}_{1}>\operatorname{rwmv}_{2}\left(0.05 \mathrm{~cm}^{-1}\right.$ $>0.04 \mathrm{~cm}^{-1}$ ), and one of the largest $N_{\text {opt }}(22)$. This level was chosen to be the "base" in the current presentation of the analysis. The unpublished results on Ir III spectrum contributed to LSDs determination.

\section{Calculations}

We used the orthogonal operators approach in our calculations. Table 4 and 5 present energy parameters obtained (or kept fixed) for the even and odd systems, respectively. The parameter values were taken from the final least squares fit (LSF) of the investigated configurations. The parameter ab initio values were calculated using the Hartree-Fock method with relativistic correction (HFR) implemented in the code developed by Cowan [34]. The ab initio HFR values of the orthogonal electrostatic parameters were calculated as in Ref. [16] from the Slater parameters given by the Cowan code. The obtained or predetermined for fixed parameters scaling factors (LSF/HFR) are shown in Tables 4 and 5.

In both the even and odd parity systems, three configurations were included in the calculations: $\left(5 \mathrm{~d}^{6}+\right.$ $\left.5 d^{5} 6 s+5 d^{4} 6 s^{2}\right)$ and $\left(5 d^{5} 6 p+5 d^{4} 6 s 6 p+5 d^{3} 6 s^{2} 6 p\right)$, respectively. The interaction between these configurations was added explicitly. The interaction integrals, parameters of unknown configurations and some parameters of the studied configurations were kept fixed at the predetermined values.

As Tables 4 and 5 indicate, the scaling factors for the strong electrostatic core operators $\mathrm{O}_{2}, \mathrm{O}_{2}{ }^{\prime}$, and spin-orbit interaction parameter $\zeta_{d}$ for the $5 \mathrm{~d}^{6}, 5 \mathrm{~d}^{5} 6 \mathrm{~s}$ and $5 \mathrm{~d}^{5} 6 \mathrm{p}$ configurations are close to each other. The parameters $E_{\alpha}$ and $E_{\beta}$ are also consistent for the three configurations, and the parameters of the 2-particle magnetic interaction of the $d s$-type $\left(A_{m s o}\right)$ in $5 \mathrm{~d}^{5} 6 \mathrm{~s}$ and of the $d p$-type $\left(Z_{l l^{\prime}}^{k}\right)$ in $5 \mathrm{~d}^{5} 6 \mathrm{p}$ configuration are well defined with a small uncertainty. These values are in agreement with those obtained in Os V and Os IV spectra $[21,22]$. The mean deviations of the fit for the even and odd systems in Ir IV are 20 and $36 \mathrm{~cm}^{-1}$, respectively. The last values are close to those in Os IV [22]: $22 \mathrm{~cm}^{-1}$ and $46 \mathrm{~cm}^{-1}$.

\section{Acknowledgements}

This work was supported by the National Institute of Standards and Technology, U.S. Department of Commerce. The spectra were acquired by Dr. S.S. Churilov of the Institute of Spectroscopy, Troitsk, Russian Federation. The initial version of the code with the orthogonal operators is kindly provided by Dr. P.H.M. Uylings and Dr. A.J.J. Raassen of the van der Waals-Zeeman Laboratorium, Universiteit van Amsterdam, The Netherlands, which is greatly appreciated. Special thanks are to Dr. A.J.J. Raassen for his help with the code.

\section{References}

[1] H. Kayser, Astroph. J. 7 (1898) 190.

[2] G.J. van het Hof, Y.N. Joshi, J.-F. Wyart and J.J. Sugar, Res. NIST 100 (1995) 687.

[3] S.S. Churilov and Y.N. Joshi, Physica Scripta 57 (1998) 556. 
[4] W.E. Albertson, Phys. Rev. 42 (1932) 443.

[5] W.E. Albertson, Phys. Rev. 54 (1938) 183.

[6] Th.A.M. van Kleef, Physica 23 (1957) 843.

[7] W.J. Childs, M. Fred, E. Schrodl and Th.A.M. van Kleef, Phys. Rev. A 10 (1974) 1028.

[8] V. Kaufman and J. Sugar, J. Opt. Soc. Am. 66 (1976) 1019.

[9] Th.A.M. van Kleef and B.C. Metsch, Physica 95C (1978) 251.

[10] R.R. Kildiyarova, S.S. Churilov and Y.N. Joshi, Physica Scripta 58 (1998) 32.

[11] V.I. Azarov, S.S. Churilov and Y.N. Joshi, Physica Scripta 60 (1999) 506.

[12] V.I. Azarov, R.R. Gayasov, Y.N. Joshi and S.S. Churilov, Physica Scripta 64 (2001) 295.

[13] R.R. Gayasov, Y.N. Joshi, A.J.J. Raassen, V. Kaufman, Physica Scripta 61 (2000) 164.

[14] P.H.M. Uylings, A.J.J. Raassen and J.-F. Wyart, J. Phys. B: At. Mol. Opt. Phys. 26 (1993) 4683.

[15] A.J.J. Raassen and P.H.M. Uylings, Physica Scripta T65 (1996) 84.

[16] P.H.M. Uylings and A.J.J. Raassen, Physica Scripta 54 (1996) 505.

[17] J.E. Hansen, B.R. Judd, A.J.J. Raassen and P.H.M. Uylings, Phys. Rev. Lett. 78 (1997) 3078.

[18] V.I. Azarov, Physica Scripta 44 (1991) 528.

[19] V.I. Azarov, Physica Scripta 48 (1993) 656.

[20] A.J.J. Raassen, V.I. Azarov, P.H.M. Uylings, Y.N. Joshi, W.-U L. Tchang-Brillet and A.N. Ryabtsev, Physica Scripta 54 (1996) 56.

[21] V.I. Azarov, A.J.J. Raassen, Y.N. Joshi, P.H.M. Uylings and A.N. Ryabtsev, Physica Scripta 56 (1997) 325.

[22] A.N. Ryabtsev, A.J.J. Raassen, W.-U L. Tchang-Brillet, Y.N. Joshi, P.H.M. Uylings and V.I. Azarov, Physica Scripta 57 (1998) 82.

[23] V.I. Azarov, A system for the automatic processing of photospectrograms, Preprint of the Institute of Spectroscopy, Russian Academy of Sciences, Troitsk, 1991, 1 - 32.

[24] A. Kramida, Yu. Ralchenko, J. Reader and NIST ASD Team, NIST Atomic Spectra Database (version 5.2), 2014, Available: http://physics.nist.gov/asd. National Institute of Standards and Technology, Gaithersburg, MD.

[25] A.E. Kramida, The program LOPT for least-squares optimization of energy levels, Computer Physics Communications 182 (2011) 419.

[26] C.E. Moore, Atomic Energy Levels, vol. III, 1958.

[27] A.E. Kramida and T. Shirai, J. Phys. Chem. Ref. Data 35 (2006) 423.

[28] J.-F. Wyart, J. Phys. B: At. Mol. Opt. Phys. 43 (2010) 4683.

[29] W.F. Meggers, M.A. Catalan and M. Sales, J. Res. Natl. Bur. Stand 61 (1958) 441.

[30] W.-U L. Tchang-Brillet and V.I. Azarov, Physica Scripta T100 (2002) 104.

[31] V.I. Azarov, J. Opt. Soc. Am. B 18 (2001) 106.

[32] J.-F. Wyart, A.J.J. Raassen, P.H.M. Uylings and Y.N. Joshi, Physica Scripta T47 (1993) 59.

[33] V.I. Azarov, W.-U L. Tchang-Brillet, J.-F. Wyart and F.G. Meijer, Physica Scripta 67 (2003) 190.

[34] R.D. Cowan, The theory of Atomic Structure and Spectra, University of California Press, Berkeley, California, 1981.

Cowan Computer Code as modified by Yu.V. Ralchenko and A.E. Kramida of the Institute of Spectroscopy, Troitsk, Russia. http://das101.troitsk.ru/COWAN

\section{Explanation of Tables}

Table 1. Classified lines in the $\left(5 d^{6}+5 d^{5} 6 s\right)-5 d^{5} 6 p$ transition array of Ir IV

gA Transition probability $\left(10^{7} \mathrm{~s}^{-1}\right)$

Int Intensity of the experimental spectral line (relative)

$\lambda \quad$ Wavelength of the experimental spectral line $(\AA)$

$v \quad$ Wavenumber of the experimental spectral line $\left(\mathrm{cm}^{-1}\right)$ 


\begin{tabular}{|c|c|c|}
\hline$\lambda_{\text {calc }}$ & \multicolumn{2}{|c|}{ Calculated (Ritz) wavelength $(\AA)$} \\
\hline Unc $\lambda_{\text {calc }}$ & \multicolumn{2}{|c|}{ Uncertainty of the calculated (Ritz) wavelength $(\AA)$} \\
\hline$\Delta(v)$ & \multicolumn{2}{|c|}{ Difference between the experimental and Ritz wavenumbers $\left(\mathrm{cm}^{-1}\right)$} \\
\hline$\Delta(\lambda)$ & \multicolumn{2}{|c|}{ Difference between the experimental and Ritz wavelengths $(\AA)$} \\
\hline $\mathrm{N}_{\text {odd }}$ & \multicolumn{2}{|c|}{$\begin{array}{l}\text { Number of observed transitions used to determine the odd level involved } \\
\text { in the transition }\end{array}$} \\
\hline $\mathrm{N}_{\text {even }}$ & \multicolumn{2}{|c|}{$\begin{array}{l}\text { Number of observed transitions used to determine the even level involved } \\
\text { in the transition }\end{array}$} \\
\hline Even level & \multicolumn{2}{|c|}{$\begin{array}{l}\text { Experimental energy value }\left(\mathrm{cm}^{-1}\right), J \text {-value (in parentheses) and the } \\
\text { configuration of the even level involved in the transition }\end{array}$} \\
\hline Odd level & \multicolumn{2}{|c|}{$\begin{array}{l}\text { Experimental energy value }\left(\mathrm{cm}^{-1}\right) \text { and } J \text {-value (in parentheses) of the odd } \\
\text { level involved in the transition }\end{array}$} \\
\hline Remark & \multicolumn{2}{|c|}{ Remarks for the identified transitions: } \\
\hline & $\mathrm{m}$ & The Ir IV line is masked by some other line \\
\hline & 200 & Expected intensity value is 200 \\
\hline & bl & The line is blended by a close line \\
\hline & $*$ & The transition has been excluded from LOP \\
\hline & $\mathrm{pa}-\mathrm{pg}$ & $\begin{array}{l}\text { Parts of the linelist with assigned wavelength uncertainties } \\
\text { (see text, Table A) }\end{array}$ \\
\hline & Ir III & $\begin{array}{l}\text { (without "bl") The line is also identified as Ir III line. The line } \\
\text { does not appear as a blend in the spectrogram, but blending is } \\
\text { possible due to a near coincidence of wavelengths. }\end{array}$ \\
\hline & Ir III (400) & $\begin{array}{l}\text { The line is also identified as Ir III line with expected intensity } \\
400\end{array}$ \\
\hline $2 \mathrm{~d}$ ord & The line has & contribution from a line seen in the $2 \mathrm{~d}$ order \\
\hline \multicolumn{3}{|c|}{ Experimental and calculated energy levels $\left(\mathrm{cm}^{-1}\right)$ in the $5 d^{6}$ and $5 d^{5} 6 \mathrm{~s}$ configurations of Ir IV } \\
\hline $\mathrm{E}_{\mathrm{obs}}$ & \multicolumn{2}{|c|}{ Observed (experimental) energy value $\left(\mathrm{cm}^{-1}\right)$} \\
\hline $\mathrm{E}_{\text {calc }}$ & \multicolumn{2}{|c|}{ Calculated from the least squares fitting (LSF) energy value $\left(\mathrm{cm}^{-1}\right)$} \\
\hline$\Delta$ & \multicolumn{2}{|c|}{ Difference between the observed and calculated energy values, $E_{o b s}-E_{c a l c}\left(\mathrm{~cm}^{-1}\right)$} \\
\hline $\mathrm{N}$ & \multicolumn{2}{|c|}{ Number of transitions used to identify the energy level } \\
\hline $\mathrm{N}_{\text {opt }}$ & \multicolumn{2}{|c|}{$\begin{array}{l}\text { Number of transitions to the level used in the LOP and WMV calculations for } \\
\text { the level (see text) }\end{array}$} \\
\hline $\mathrm{rwmv}_{1}$ & \multicolumn{2}{|c|}{ Square root value of the weighted mean variance given by the equation (7) } \\
\hline $\mathrm{rwmv}_{2}$ & \multicolumn{2}{|c|}{$\begin{array}{l}\text { Square root value of the weighted mean variance given by the equation (8), } \\
\text { except for the levels determined by a single line (see text). }\end{array}$} \\
\hline Unc & \multicolumn{2}{|c|}{$\begin{array}{l}\text { Uncertainty of the level with respect to the "base" even level with } J=4 \text { at } \\
71191.62 \mathrm{~cm}^{-1} \text { (see text). }\end{array}$} \\
\hline Composition & \multicolumn{2}{|c|}{$\begin{array}{l}L S \text {-composition of the level. The number preceding the symbol "|" and the } \\
\text { term name has the following meaning: } 1 \text { stands for } 5 \mathrm{~d}^{6} \text { and } 2 \text { stands for } 5 \mathrm{~d}^{5} 6 \mathrm{~s} \text {. }\end{array}$} \\
\hline
\end{tabular}

Table 3. Experimental and calculated energy levels $\left(\mathrm{cm}^{-1}\right)$ in the $5 d^{5} 6 p$ and $5 d^{4} 6 s 6 p$ configurations of Ir IV

Calculated levels of the $5 d^{4} 6 s 6 p$ configuration that are inside the energy range of the $5 d^{5} 6 p$ configuration are given. Other $5 \mathrm{~d}^{4} 6 \mathrm{~s} 6 \mathrm{p}$ levels are omitted.

$\begin{array}{ll}\mathrm{E}_{\mathrm{obs}} & \text { Observed (experimental) energy value }\left(\mathrm{cm}^{-1}\right) \\ \mathrm{E}_{\text {calc }} & \text { Calculated from the least squares fitting (LSF) energy value }\left(\mathrm{cm}^{-1}\right) \\ \Delta & \text { Difference between the observed and calculated energy values, } \mathrm{E}_{\text {obs }}-\mathrm{E}_{\text {calc }}\left(\mathrm{cm}^{-1}\right) \\ \mathrm{N} & \text { Number of transitions used to identify the energy level } \\ \mathrm{N}_{\mathrm{opt}} & \begin{array}{l}\text { Number of transitions from the level used in the LOP and WMV calculations for } \\ \text { the level (see text). }\end{array} \\ \mathrm{rwmv}_{1} & \text { Square root value of the weighted mean variance given by the equation (7) } \\ \mathrm{rwmv}_{2} & \text { Square root value of the weighted mean variance given by the equation (8), }\end{array}$


except for the levels determined by a single line (see text).

Unc Uncertainty of the level with respect to the "base" even level with $J=4$ at $71191.62 \mathrm{~cm}^{-1}$ (see text).

Composition $\quad L S$-composition of the level. The number preceding the symbol "|" and the term name has the following meaning: 1 stands for $5 \mathrm{~d}^{5} 6 \mathrm{p}$ and 2 stands for $5 d^{4} 6 s 6 p$.

Table 4. Fitted and calculated parameter values $\left(\mathrm{cm}^{-1}\right)$ in the $5 d^{6}, 5 d^{5} 6 \mathrm{~s}$ and $5 d^{4} 6 s^{2}$ configurations of Ir IV

Parameter Parameter name

Fitted value Parameter value $\left(\mathrm{cm}^{-1}\right)$ obtained in the final least squares fitting (LSF). Uncertainty of the fitted parameter value is given in parentheses.

HFR Ab initio value of the parameter. It was calculated using the Hartree-Fock method with relativistic correction (HFR) implemented in the code developed by Cowan [34].

Fitted/HFR Obtained (or predetermined for fixed parameters) scaling factors

Table 5. Fitted and calculated parameter values $\left(\mathrm{cm}^{-1}\right)$ in the $5 d^{5} 6 p, 5 d^{4} 6 s 6 p$ and $5 d^{3} 6 s^{2} 6 p$ configurations of Ir IV

Parameter Parameter name

Fitted value Parameter value $\left(\mathrm{cm}^{-1}\right)$ obtained in the final least squares fitting (LSF). Uncertainty of the fitted parameter value is given in parentheses.

HFR Ab initio value of the parameter. It was calculated using the Hartree-Fock method with relativistic correction (HFR) implemented in the code developed by Cowan [34].

Fitted/HFR Obtained (or predetermined for fixed parameters) scaling factors 
Table 1

Classified lines in the $\left(5 d^{6}+5 d^{5} 6 s\right)-5 d^{5} 6 p$ transition array of Ir IV.

\begin{tabular}{|c|c|c|c|c|c|c|c|c|c|c|c|c|c|}
\hline$\underset{\left(10^{7} \mathrm{~s}^{-1}\right)}{\mathrm{gA}}$ & Int & $\begin{array}{c}\lambda \\
(\AA)\end{array}$ & $\begin{array}{c}v \\
\left(\mathrm{~cm}^{-1}\right)\end{array}$ & $\begin{array}{c}\lambda \text { calc } \\
(\AA)\end{array}$ & $\begin{array}{c}\text { Unc } \lambda \text { calc } \\
(\AA)\end{array}$ & $\begin{array}{l}\Delta(\mathrm{v}) \\
\left(\mathrm{cm}^{-1}\right)\end{array}$ & $\begin{array}{l}\Delta(\lambda) \\
(\AA)\end{array}$ & $\mathrm{N}_{\text {odd }}$ & $\mathrm{N}_{\text {ever }}$ & & a level & Odd level & Remark \\
\hline 10 & 15 & 651.836 & 153412.70 & 651.8389 & 0.0009 & 0.57 & -0.002 & 14 & 26 & $5 d^{6}$ & $0.00(4)$ & 153412.13(4) & pa* \\
\hline 181 & 77 & 657.898 & 151999.34 & 657.8899 & 0.0023 & -1.79 & 0.008 & 3 & 36 & $5 d^{6}$ & $27940.65(4)$ & 179941.78(3) & pa \\
\hline 26 & 20 & 660.754 & 151342.30 & 660.7501 & 0.0011 & -0.84 & 0.004 & 11 & 39 & $5 d^{6}$ & $5824.11(3)$ & $157167.25(4)$ & pa* \\
\hline 58 & 72 & 663.927 & 150619.08 & 663.9277 & 0.0011 & 0.26 & -0.001 & 7 & 23 & $5 d^{6}$ & 19096.89(6) & 169715.71(6) & pa \\
\hline 25 & 17 & 671.026 & 149025.61 & 671.0151 & 0.0019 & -2.34 & 0.011 & 12 & 39 & $5 d^{6}$ & $5824.11(3)$ & $154852.06(2)$ & pa* \\
\hline 101 & 52 & 672.680 & 148659.07 & 672.6791 & 0.0012 & -0.22 & 0.001 & 8 & 32 & $5 d^{6}$ & $14546.32(4)$ & 163205.61(3) & pa \\
\hline 76 & 90 & 675.607 & 148015.00 & 675.6078 & 0.0014 & 0.13 & -0.001 & 9 & 30 & $5 d^{6}$ & $5178.26(2)$ & $153193.13(2)$ & pa \\
\hline 80 & 85 & 677.571 & 147586.01 & 677.5618 & 0.0009 & -2.01 & 0.009 & 14 & 39 & $5 d^{6}$ & $5824.11(3)$ & $153412.13(4)$ & pa * \\
\hline 83 & 100 & 679.363 & 147196.63 & 679.3692 & 0.0012 & 1.26 & -0.006 & 13 & 30 & $5 d^{6}$ & $5178.26(2)$ & 152373.63(3) & pa \\
\hline 250 & 277 & 681.081 & 146825.50 & 681.0767 & 0.0015 & -0.84 & 0.004 & 4 & 23 & $5 d^{6}$ & 19096.89(6) & $165923.23(6)$ & $\mathrm{pa}$ \\
\hline 59 & 130 & 681.688 & 146694.57 & 681.6918 & 0.0020 & 0.72 & -0.003 & 3 & 30 & $5 d^{6}$ & $5178.26(2)$ & 151872.11(1) & $\mathrm{pa}$ \\
\hline 109 & 165 & 682.356 & 146551.00 & 682.3632 & 0.0011 & 1.48 & -0.007 & 13 & 39 & $5 d^{6}$ & $5824.11(3)$ & 152373.63(3) & $\mathrm{pa}$ \\
\hline 184 & 475 & 683.609 & 146282.43 & 683.6190 & 0.0013 & 2.12 & -0.010 & 8 & 37 & $5 d^{6}$ & $18463.42(5)$ & 164743.73(4) & pa \\
\hline 296 & 500 & 687.122 & 145534.63 & 687.1260 & 0.0011 & 0.91 & -0.004 & 8 & 37 & $5 d^{6}$ & 18463.42(5) & 163997.14(4) & $\mathrm{pa}$ \\
\hline 148 & 130 & 687.169 & 145524.65 & 687.1660 & 0.0010 & -0.60 & 0.003 & 17 & 26 & $5 d^{6}$ & $0.00(4)$ & $145525.25(5)$ & $\mathrm{pa}$ \\
\hline 105 & 295 & 689.634 & 145004.49 & 689.6372 & 0.0009 & 0.71 & -0.003 & 19 & 26 & $5 d^{6}$ & $0.00(4)$ & $145003.78(4)$ & pa \\
\hline 53 & 240 & 691.531 & 144606.68 & 691.5329 & 0.0018 & 0.41 & -0.002 & 8 & 25 & $5 d^{6}$ & $19128.73(2)$ & $163735.00(3)$ & $\mathrm{pa} * 100$ \\
\hline 49 & 105 & 692.468 & 144410.92 & 692.4681 & 0.0011 & -0.06 & 0.000 & 11 & 39 & $5 d^{6}$ & 5824.11(3) & $150235.09(3)$ & $\mathrm{pa} *$ \\
\hline 354 & 1050 & 696.587 & 143557.05 & 696.5872 & 0.0011 & 0.00 & 0.000 & 11 & 23 & $5 d^{6}$ & $19096.89(6)$ & 162653.94(5) & pa \\
\hline 106 & 277 & 697.159 & 143439.28 & 697.1625 & 0.0011 & 0.69 & -0.003 & 9 & 37 & $5 d^{6}$ & $18463.42(5)$ & 161902.01(4) & pa \\
\hline 43 & 57 & 698.421 & 143180.10 & 698.4312 & 0.0022 & 2.08 & -0.010 & 7 & 25 & $5 d^{6}$ & 22138.98(2) & $165317.00(2)$ & pa* \\
\hline 30 & 15 & 700.418 & 142771.95 & 700.4227 & 0.0019 & 1.03 & -0.005 & 7 & 25 & $5 d^{6}$ & $22138.98(2)$ & $164909.90(2)$ & pa* \\
\hline 145 & 1312 & 703.214 & 142204.30 & 703.2409 & 0.0010 & 5.52 & -0.027 & 13 & 26 & $5 d^{6}$ & $0.00(4)$ & $142198.78(4)$ & $\mathrm{pa} * 700, \mathrm{bl}$ \\
\hline 163 & 310 & 703.414 & 142163.85 & 703.4155 & 0.0011 & 0.37 & -0.002 & 10 & 30 & $5 d^{6}$ & $5178.26(2)$ & 147341.74(3) & pa \\
\hline 237 & 735 & 704.536 & 141937.47 & 704.5275 & 0.0010 & -1.64 & 0.008 & 11 & 39 & $5 d^{6}$ & $5824.11(3)$ & $147763.22(4)$ & $\mathrm{pa}$ \\
\hline 175 & 400 & 705.506 & 141742.30 & 705.5043 & 0.0015 & -0.29 & 0.001 & 9 & 36 & $5 d^{6}$ & $27940.65(4)$ & 169683.24(3) & pa \\
\hline 44 & 60 & 706.469 & 141548.95 & 706.4649 & 0.0012 & -0.89 & 0.004 & 8 & 36 & $5 d^{6}$ & 27940.65(4) & $169490.49(5)$ & pa* \\
\hline 277 & 1517 & 706.626 & 141517.54 & 706.6257 & 0.0011 & -0.09 & 0.000 & 10 & 39 & $5 d^{6}$ & $5824.11(3)$ & 147341.74(3) & pa \\
\hline 128 & 127 & 707.722 & 141298.46 & 707.7177 & 0.0013 & -0.83 & 0.004 & 8 & 37 & $5 d^{6}$ & $18463.42(5)$ & 159762.71(5) & $\mathrm{pa}$ \\
\hline 181 & 1320 & 708.508 & 141141.77 & 708.5105 & 0.0011 & 0.61 & -0.003 & 15 & 26 & $5 d^{6}$ & $0.00(4)$ & 141141.16(4) & $\mathrm{pa}$ \\
\hline 390 & 795 & 708.901 & 141063.50 & 708.8998 & 0.0011 & -0.16 & 0.001 & 7 & 32 & $5 d^{6}$ & $28652.05(5)$ & 169715.71(6) & $\mathrm{pa}$ \\
\hline 96 & 125 & 708.965 & 141050.75 & 708.9605 & 0.0011 & -0.84 & 0.004 & 10 & 32 & $5 d^{6}$ & $14546.32(4)$ & 155597.91(4) & pa \\
\hline 46 & 305 & 709.494 & 140945.62 & 709.4969 & 0.0023 & 0.68 & -0.003 & 5 & 39 & $5 d^{6}$ & 21190.03(3) & $162134.97(2)$ & 100 \\
\hline 251 & 1257 & 711.549 & 140538.40 & 711.5478 & 0.0013 & -0.30 & 0.002 & 13 & 32 & $5 d^{6}$ & $14546.32(4)$ & $155085.02(3)$ & pa 400 \\
\hline 8 & 25 & 712.451 & 140360.61 & 712.4432 & 0.0011 & -1.46 & 0.007 & 17 & 39 & $5 d^{6}$ & $5824.11(3)$ & 146186.18(3) & pa* \\
\hline 49 & 40 & 713.384 & 140177.00 & 713.3753 & 0.0031 & -1.66 & 0.008 & 6 & 39 & $5 d^{6}$ & 21190.03(3) & $161368.69(3)$ & pa* \\
\hline 59 & 42 & 714.124 & 140031.80 & 714.1221 & 0.0017 & -0.28 & 0.001 & 8 & 25 & $5 d^{6}$ & $19128.73(2)$ & $159160.81(2)$ & pa \\
\hline 131 & 1550 & 714.298 & 139997.69 & 714.2940 & 0.0010 & -0.69 & 0.004 & 15 & 26 & $5 d^{6}$ & $0.00(4)$ & 139998.38(4) & $\mathrm{pa}$ \\
\hline
\end{tabular}




\begin{tabular}{|c|c|c|c|c|c|c|c|c|c|c|c|c|c|c|}
\hline 91 & 255 & 714.934 & 139873.15 & 714.9346 & 0.0018 & 0.21 & -0.001 & 9 & 39 & $5 d^{6}$ & 21190.03(3) & $161062.97(2)$ & pa & \\
\hline 306 & 1575 & 715.617 & 139739.48 & 715.6157 & 0.0010 & -0.33 & 0.002 & 16 & 26 & $5 d^{6}$ & $0.00(4)$ & $139739.81(5)$ & pa & \\
\hline 45 & 107 & 715.692 & 139725.01 & 715.6920 & 0.0011 & 0.10 & -0.001 & 12 & 37 & $5 d^{6}$ & $18463.42(5)$ & $158188.33(4)$ & pa & \\
\hline 142 & 600 & 716.609 & 139546.07 & 716.6144 & 0.0017 & 1.01 & -0.005 & 5 & 25 & $5 d^{6}$ & $22138.98(2)$ & $161684.04(1)$ & $\mathrm{pa}$ & 300 \\
\hline 164 & 1377 & 717.972 & 139281.10 & 717.9670 & 0.0011 & -1.06 & 0.005 & 12 & 32 & $5 d^{6}$ & $14546.32(4)$ & $153828.48(4)$ & pa & 400 \\
\hline 156 & 1780 & 719.018 & 139078.48 & 719.0173 & 0.0023 & -0.24 & 0.001 & 7 & 17 & $5 d^{6}$ & $26238.28(1)$ & $165317.00(2)$ & pa & 500 \\
\hline 174 & 262 & 719.262 & 139031.32 & 719.2638 & 0.0010 & 0.28 & -0.001 & 15 & 39 & $5 d^{6}$ & $5824.11(3)$ & $144855.15(3)$ & pa & \\
\hline 77 & 57 & 719.820 & 138923.56 & 719.8181 & 0.0019 & -0.43 & 0.002 & 9 & 25 & $5 d^{6}$ & $22138.98(2)$ & $161062.97(2)$ & pa & \\
\hline 248 & 880 & 720.135 & 138862.76 & 720.1271 & 0.0011 & -1.61 & 0.008 & 11 & 37 & $5 d^{6}$ & $18463.42(5)$ & $157327.79(6)$ & pa & \\
\hline 26 & 37 & 720.452 & 138801.77 & 720.4505 & 0.0011 & -0.27 & 0.001 & 9 & 39 & $5 d^{6}$ & $5824.11(3)$ & $144626.15(2)$ & pa* & \\
\hline 563 & 1352 & 720.875 & 138720.30 & 720.8766 & 0.0016 & 0.30 & -0.002 & 5 & 30 & $5 d^{6}$ & $25969.65(4)$ & $164689.65(3)$ & pa & \\
\hline 57 & 20 & 721.484 & 138603.17 & 721.4920 & 0.0017 & 1.50 & -0.008 & 8 & 39 & $5 d^{6}$ & $21190.03(3)$ & $159791.70(4)$ & pa* & \\
\hline 388 & 1705 & 721.864 & 138530.33 & 721.8663 & 0.0011 & 0.52 & -0.003 & 10 & 26 & $5 d^{6}$ & $0.00(4)$ & $138529.81(3)$ & pa & \\
\hline 232 & 405 & 722.259 & 138454.57 & 722.2549 & 0.0012 & -0.71 & 0.004 & 7 & 32 & $5 d^{6}$ & $28652.05(5)$ & $167107.33(4)$ & pa & \\
\hline 42 & 42 & 722.339 & 138439.14 & 722.3378 & 0.0019 & -0.26 & 0.001 & 6 & 39 & $5 d^{6}$ & $21190.03(3)$ & $159629.42(3)$ & pa* & \\
\hline 1680 & 1875 & 722.625 & 138384.27 & 722.6261 & 0.0011 & 0.11 & -0.001 & 12 & 23 & $5 d^{6}$ & $19096.89(6)$ & $157481.05(5)$ & pa & \\
\hline 179 & 492 & 722.845 & 138342.19 & 722.8459 & 0.0018 & 0.12 & -0.001 & 5 & 36 & $5 d^{6}$ & $27940.65(4)$ & $166282.72(5)$ & pa & \\
\hline 330 & 385 & 723.464 & 138223.86 & 723.4619 & 0.0020 & -0.42 & 0.002 & 3 & 32 & $5 d^{6}$ & $28652.05(5)$ & $166876.33(4)$ & pa & Ir V \\
\hline 131 & 100 & 724.502 & 138025.94 & 724.4934 & 0.0013 & -1.55 & 0.008 & 8 & 30 & $5 d^{6}$ & $25969.65(4)$ & $163997.14(4)$ & pa* & \\
\hline 216 & 242 & 724.794 & 137970.33 & 724.7911 & 0.0016 & -0.45 & 0.002 & 8 & 39 & $5 d^{6}$ & $21190.03(3)$ & $159160.81(2)$ & pa & \\
\hline 240 & 747 & 725.876 & 137764.56 & 725.8719 & 0.0019 & -0.79 & 0.004 & 8 & 30 & $5 d^{6}$ & $25969.65(4)$ & $163735.00(3)$ & pa* & 200 \\
\hline 156 & 725 & 725.918 & 137756.55 & 725.9100 & 0.0010 & -1.58 & 0.008 & 15 & 32 & $5 d^{6}$ & $14546.32(4)$ & $152304.45(5)$ & pa & \\
\hline 84 & 322 & 726.289 & 137686.22 & 726.2858 & 0.0012 & -0.62 & 0.003 & 13 & 30 & $5 d^{6}$ & $5178.26(2)$ & $142865.10(2)$ & pa & \\
\hline 36 & 27 & 727.332 & 137488.72 & 727.3233 & 0.0021 & -1.72 & 0.009 & 6 & 25 & $5 d^{6}$ & $22138.98(2)$ & $159629.42(3)$ & pa $*$ & \\
\hline 386 & 560 & 728.257 & 137314.12 & 728.2533 & 0.0017 & -0.73 & 0.004 & 6 & 12 & $5 d^{6}$ & $33303.67(6)$ & $170618.53(5)$ & pa & \\
\hline 237 & 1737 & 728.455 & 137276.80 & 728.4522 & 0.0011 & -0.56 & 0.003 & 9 & 26 & $5 d^{6}$ & $0.00(4)$ & $137277.36(4)$ & pa & \\
\hline 267 & 592 & 728.676 & 137235.17 & 728.6720 & 0.0014 & -0.79 & 0.004 & 8 & 30 & $5 d^{6}$ & $25969.65(4)$ & $163205.61(3)$ & pa & \\
\hline 372 & 1237 & 729.211 & 137134.54 & 729.2112 & 0.0011 & 0.05 & 0.000 & 10 & 37 & $5 d^{6}$ & $18463.42(5)$ & $155597.91(4)$ & pa & \\
\hline 101 & 550 & 729.711 & 137040.55 & 729.7087 & 0.0012 & -0.44 & 0.002 & 13 & 39 & $5 d^{6}$ & $5824.11(3)$ & $142865.10(2)$ & pa & \\
\hline 119 & 397 & 729.814 & 137021.23 & 729.8107 & 0.0016 & -0.60 & 0.003 & 8 & 25 & $5 d^{6}$ & $22138.98(2)$ & $159160.81(2)$ & pa & \\
\hline 37 & 25 & 729.934 & 136998.59 & 729.9361 & 0.0011 & 0.29 & -0.002 & 12 & 39 & $5 d^{6}$ & $21190.03(3)$ & $158188.33(4)$ & pa* & \\
\hline 73 & 37 & 730.980 & 136802.70 & 730.9777 & 0.0014 & -0.38 & 0.002 & 8 & 36 & $5 d^{6}$ & $27940.65(4)$ & $164743.73(4)$ & pa* & \\
\hline 142 & 315 & 731.273 & 136747.78 & 731.2668 & 0.0016 & -1.22 & 0.007 & 5 & 36 & $5 d^{6}$ & $27940.65(4)$ & $164689.65(3)$ & pa & \\
\hline 1253 & 1622 & 731.545 & 136697.01 & 731.5440 & 0.0011 & -0.18 & 0.001 & 12 & 37 & $5 d^{6}$ & $18463.42(5)$ & $155160.61(5)$ & pa & \\
\hline 228 & 1230 & 732.133 & 136587.11 & 732.1311 & 0.0013 & -0.44 & 0.002 & 6 & 32 & $5 d^{6}$ & $28652.05(5)$ & $165239.60(5)$ & $\mathrm{pa}$ & \\
\hline 341 & 1502 & 732.979 & 136429.50 & 732.9804 & 0.0011 & 0.21 & -0.001 & 12 & 26 & $5 d^{6}$ & $0.00(4)$ & $136429.29(5)$ & pa & \\
\hline 33 & 75 & 733.277 & 136374.17 & 733.2740 & 0.0010 & -0.50 & 0.003 & 13 & 39 & $5 d^{6}$ & $5824.11(3)$ & $142198.78(4)$ & pa & \\
\hline 74 & 125 & 733.371 & 136356.56 & 733.3676 & 0.0023 & -0.71 & 0.004 & 7 & 43 & $5 d^{6}$ & $28959.73(3)$ & $165317.00(2)$ & pa & \\
\hline 34 & 332 & 733.734 & 136289.23 & 733.7376 & 0.0015 & 0.72 & -0.004 & 5 & 30 & $5 d^{6}$ & $5178.26(2)$ & $141466.77(2)$ & pa & \\
\hline 1328 & 1855 & 734.283 & 136187.22 & 734.2854 & 0.0017 & 0.40 & -0.002 & 8 & 12 & $5 d^{6}$ & $33303.67(6)$ & $169490.49(5)$ & pa & \\
\hline 657 & 922 & 734.799 & 136091.71 & 734.7988 & 0.0014 & 0.03 & 0.000 & 8 & 32 & $5 d^{6}$ & $28652.05(5)$ & $164743.73(4)$ & $\mathrm{pa}$ & \\
\hline 1336 & 1985 & 734.951 & 136063.57 & 734.9498 & 0.0012 & -0.15 & 0.001 & 12 & 23 & $5 d^{6}$ & $19096.89(6)$ & $155160.61(5)$ & pa & \\
\hline 104 & 210 & 735.094 & 136037.06 & 735.0990 & 0.0011 & 0.96 & -0.005 & 14 & 30 & $5 d^{6}$ & $5178.26(2)$ & $141214.36(3)$ & pa & \\
\hline 27 & 62 & 735.416 & 135977.44 & 735.4173 & 0.0014 & 0.22 & -0.001 & 11 & 39 & $5 d^{6}$ & $21190.03(3)$ & $157167.25(4)$ & pa $*$ & \\
\hline
\end{tabular}




\begin{tabular}{|c|c|c|c|c|c|c|c|c|c|c|c|c|c|c|}
\hline 143 & 60 & 735.563 & 135950.36 & 735.5636 & 0.0020 & 0.19 & -0.001 & 7 & 43 & $5 d^{6}$ & 28959.73(3) & $164909.90(2)$ & $\mathrm{pa}$ & \\
\hline 818 & 1805 & 735.658 & 135932.67 & 735.6600 & 0.0013 & 0.31 & -0.002 & 9 & 30 & $5 d^{6}$ & $25969.65(4)$ & $161902.01(4)$ & pa & \\
\hline 51 & 60 & 736.260 & 135821.66 & 736.2640 & 0.0016 & 0.82 & -0.004 & 7 & 14 & $5 d^{6}$ & $8526.56(1)$ & $144347.40(1)$ & pa & \\
\hline 451 & 1050 & 736.406 & 135794.66 & 736.4077 & 0.0019 & 0.31 & -0.002 & 8 & 36 & $5 d^{6}$ & $27940.65(4)$ & $163735.00(3)$ & pa & \\
\hline 373 & 1147 & 736.748 & 135731.64 & 736.7572 & 0.0016 & 1.72 & -0.009 & 5 & 43 & $5 d^{6}$ & 28959.73(3) & $164689.65(3)$ & pa & \\
\hline 108 & 465 & 737.136 & 135660.21 & 737.1351 & 0.0019 & -0.14 & 0.001 & 7 & 7 & $5 d^{6}$ & $8687.05(0)$ & $144347.40(1)$ & pa & \\
\hline 212 & 1152 & 737.216 & 135645.55 & 737.2312 & 0.0016 & 2.89 & -0.016 & 5 & 39 & $5 d^{6}$ & $5824.11(3)$ & $141466.77(2)$ & pa * & $900, \mathrm{bl}$ \\
\hline 1242 & 1942 & 737.486 & 135595.83 & 737.4879 & 0.0011 & 0.39 & -0.002 & 17 & 26 & $5 d^{6}$ & $0.00(4)$ & $135595.44(4)$ & pa & \\
\hline 222 & 1147 & 738.001 & 135501.10 & 738.0041 & 0.0012 & 0.50 & -0.003 & 8 & 30 & $5 d^{6}$ & $5178.26(2)$ & $140678.86(1)$ & pa & \\
\hline 300 & 730 & 738.301 & 135446.18 & 738.3029 & 0.0018 & 0.42 & -0.002 & 5 & 17 & $5 d^{6}$ & $26238.28(1)$ & $161684.04(1)$ & pa & \\
\hline 185 & 1237 & 738.444 & 135419.92 & 738.4432 & 0.0011 & -0.11 & 0.001 & 15 & 26 & $5 d^{6}$ & $0.00(4)$ & $135420.03(3)$ & pa & \\
\hline 477 & 992 & 738.562 & 135398.32 & 738.5577 & 0.0030 & -0.72 & 0.004 & 6 & 30 & $5 d^{6}$ & $25969.65(4)$ & $161368.69(3)$ & pa & \\
\hline 79 & 240 & 738.611 & 135389.17 & 738.6056 & 0.0011 & -1.08 & 0.006 & 14 & 39 & $5 d^{6}$ & $5824.11(3)$ & $141214.36(3)$ & pa & \\
\hline 24 & 95 & 738.841 & 135347.10 & 738.8326 & 0.0010 & -1.56 & 0.009 & 15 & 30 & $5 d^{6}$ & $5178.26(2)$ & $140526.92(3)$ & pa & \\
\hline 176 & 1105 & 739.006 & 135316.92 & 739.0052 & 0.0010 & -0.13 & 0.001 & 15 & 39 & $5 d^{6}$ & $5824.11(3)$ & $141141.16(4)$ & pa & \\
\hline 91 & 460 & 739.436 & 135238.14 & 739.4306 & 0.0013 & -1.06 & 0.006 & 11 & 39 & $5 d^{6}$ & $21190.03(3)$ & $156429.22(2)$ & pa & \\
\hline 364 & 1137 & 739.668 & 135195.86 & 739.6693 & 0.0011 & 0.31 & -0.002 & 12 & 37 & $5 d^{6}$ & $18463.42(5)$ & $153658.98(5)$ & pa & \\
\hline 462 & 1702 & 740.511 & 135041.97 & 740.5355 & 0.0013 & 4.56 & -0.025 & 8 & 43 & $5 d^{6}$ & 28959.73(3) & $163997.14(4)$ & pa * & 800, bl \\
\hline 185 & 470 & 740.612 & 135023.56 & 740.6101 & 0.0014 & -0.26 & 0.001 & 13 & 25 & $5 d^{6}$ & $19128.73(2)$ & $154152.55(3)$ & pa & \\
\hline 129 & 400 & 741.028 & 134947.76 & 741.0223 & 0.0011 & -0.95 & 0.005 & 14 & 37 & $5 d^{6}$ & $18463.42(5)$ & $153412.13(4)$ & pa & \\
\hline 68 & 60 & 741.706 & 134824.40 & 741.7039 & 0.0021 & -0.29 & 0.002 & 9 & 17 & $5 d^{6}$ & $26238.28(1)$ & $161062.97(2)$ & pa & \\
\hline 24 & 42 & 741.966 & 134777.12 & 741.9759 & 0.0020 & 1.85 & -0.010 & 8 & 43 & $5 d^{6}$ & $28959.73(3)$ & $163735.00(3)$ & pa * & \\
\hline 235 & 422 & 742.318 & 134713.21 & 742.3173 & 0.0011 & -0.08 & 0.000 & 11 & 36 & $5 d^{6}$ & $27940.65(4)$ & $162653.94(5)$ & pa & \\
\hline 146 & 537 & 742.358 & 134705.88 & 742.3689 & 0.0015 & 1.96 & -0.011 & 8 & 30 & $5 d^{6}$ & $27670.52(2)$ & $162374.44(3)$ & pa * & 200 \\
\hline 48 & 90 & 742.808 & 134624.24 & 742.8115 & 0.0024 & 0.57 & -0.003 & 7 & 31 & $5 d^{6}$ & $30693.33(3)$ & $165317.00(2)$ & pa & \\
\hline 467 & 1002 & 743.000 & 134589.45 & 743.0044 & 0.0014 & 0.75 & -0.004 & 7 & 32 & $5 d^{6}$ & $28652.05(5)$ & $163240.75(5)$ & pa & \\
\hline 433 & 1117 & 743.152 & 134561.92 & 743.1514 & 0.0012 & -0.17 & 0.001 & 12 & 23 & $5 d^{6}$ & $19096.89(6)$ & $153658.98(5)$ & pa & \\
\hline 568 & 880 & 743.685 & 134465.55 & 743.6910 & 0.0030 & 1.10 & -0.006 & 5 & 30 & $5 d^{6}$ & $27670.52(2)$ & $162134.97(2)$ & pa & \\
\hline 119 & 127 & 743.861 & 134433.81 & 743.8606 & 0.0015 & 0.02 & 0.000 & 8 & 36 & $5 d^{6}$ & $27940.65(4)$ & $162374.44(3)$ & pa & \\
\hline 154 & 357 & 744.001 & 134408.39 & 744.0040 & 0.0011 & 0.51 & -0.003 & 10 & 39 & $5 d^{6}$ & 21190.03(3) & $155597.91(4)$ & pa & \\
\hline 110 & 415 & 744.077 & 134394.64 & 744.0952 & 0.0012 & 3.24 & -0.018 & 12 & 32 & $5 d^{6}$ & $14546.32(4)$ & $148937.72(5)$ & pa * & 200 \\
\hline 463 & 1707 & 744.383 & 134339.41 & 744.3880 & 0.0015 & 0.87 & -0.005 & 13 & 14 & $5 d^{6}$ & $8526.56(1)$ & $142865.10(2)$ & pa & \\
\hline 170 & 105 & 744.916 & 134243.32 & 744.9169 & 0.0017 & 0.15 & -0.001 & 9 & 29 & $5 d^{6}$ & $35440.07(3)$ & $169683.24(3)$ & pa & \\
\hline 81 & 290 & 744.988 & 134230.37 & 744.9875 & 0.0010 & -0.07 & 0.000 & 11 & 39 & $5 d^{6}$ & $5824.11(3)$ & $140054.55(3)$ & pa & \\
\hline 807 & 985 & 745.061 & 134217.14 & 745.0645 & 0.0021 & 0.58 & -0.003 & 7 & 31 & $5 d^{6}$ & $30693.33(3)$ & $164909.90(2)$ & pa & \\
\hline 320 & 325 & 745.185 & 134194.85 & 745.1874 & 0.0020 & 0.41 & -0.002 & 3 & 12 & $5 d^{6}$ & $33303.67(6)$ & $167498.11(7)$ & pa & \\
\hline 783 & 1465 & 745.739 & 134095.23 & 745.7372 & 0.0015 & -0.27 & 0.002 & 6 & 23 & $5 d^{6}$ & $19096.89(6)$ & $153192.39(7)$ & pa & \\
\hline 35 & 15 & 745.905 & 134065.28 & 745.9102 & 0.0017 & 0.88 & -0.005 & 9 & 25 & $5 d^{6}$ & $19128.73(2)$ & $153193.13(2)$ & pa * & \\
\hline 280 & 400 & 745.988 & 134050.34 & 745.9881 & 0.0015 & -0.05 & 0.000 & 8 & 31 & $5 d^{6}$ & $30693.33(3)$ & $164743.73(4)$ & pa & \\
\hline 530 & 1872 & 746.129 & 134025.15 & 746.1277 & 0.0011 & -0.16 & 0.001 & 16 & 26 & $5 d^{6}$ & $0.00(4)$ & $134025.31(4)$ & pa & \\
\hline 157 & 175 & 746.262 & 134001.11 & 746.2581 & 0.0012 & -0.78 & 0.004 & 11 & 32 & $5 d^{6}$ & $28652.05(5)$ & $162653.94(5)$ & pa & \\
\hline 677 & 1197 & 746.485 & 133961.20 & 746.4839 & 0.0013 & -0.16 & 0.001 & 9 & 36 & $5 d^{6}$ & $27940.65(4)$ & $161902.01(4)$ & pa & \\
\hline 441 & 1170 & 746.852 & 133895.30 & 746.8539 & 0.0014 & 0.31 & -0.002 & 13 & 39 & $5 d^{6}$ & $21190.03(3)$ & $155085.02(3)$ & pa & \\
\hline 116 & 677 & 747.158 & 133840.46 & 747.1551 & 0.0010 & -0.56 & 0.003 & 15 & 37 & $5 d^{6}$ & $18463.42(5)$ & $152304.45(5)$ & pa & 300 \\
\hline
\end{tabular}




\begin{tabular}{|c|c|c|c|c|c|c|c|c|c|c|c|c|c|c|}
\hline 485 & 1660 & 747.313 & 133812.76 & 747.3146 & 0.0013 & 0.30 & -0.002 & 8 & 32 & $5 d^{6}$ & $14546.32(4)$ & $148358.78(3)$ & pa & \\
\hline 11 & 55 & 748.151 & 133662.77 & 748.1556 & 0.0023 & 0.74 & -0.004 & 12 & 39 & $5 d^{6}$ & $21190.03(3)$ & $154852.06(2)$ & pa $*$ & \\
\hline 168 & 870 & 749.424 & 133435.79 & 749.4325 & 0.0011 & 1.49 & -0.008 & 8 & 30 & $5 d^{6}$ & $5178.26(2)$ & $138612.56(2)$ & pa & \\
\hline 222 & 1707 & 749.554 & 133412.60 & 749.5534 & 0.0011 & -0.18 & 0.001 & 8 & 26 & $5 d^{6}$ & $0.00(4)$ & $133412.78(5)$ & pa & \\
\hline 60 & 120 & 749.656 & 133394.45 & 749.6676 & 0.0020 & 2.00 & -0.011 & 9 & 30 & $5 d^{6}$ & $27670.52(2)$ & $161062.97(2)$ & $\mathrm{pa} *$ & \\
\hline 125 & 925 & 749.896 & 133351.86 & 749.8975 & 0.0011 & 0.31 & -0.002 & 10 & 30 & $5 d^{6}$ & $5178.26(2)$ & $138529.81(3)$ & pa & \\
\hline 77 & 50 & 749.979 & 133337.08 & 749.9717 & 0.0012 & -1.27 & 0.007 & 11 & 32 & $5 d^{6}$ & $14546.32(4)$ & $147884.68(4)$ & pa & \\
\hline 158 & 290 & 750.491 & 133246.01 & 750.4978 & 0.0014 & 1.11 & -0.006 & 13 & 25 & $5 d^{6}$ & $19128.73(2)$ & $152373.63(3)$ & pa & \\
\hline 756 & 1455 & 750.659 & 133216.37 & 750.6555 & 0.0011 & -0.53 & 0.003 & 11 & 32 & $5 d^{6}$ & $14546.32(4)$ & $147763.22(4)$ & pa & \\
\hline 388 & 697 & 750.709 & 133207.44 & 750.7081 & 0.0012 & -0.12 & 0.001 & 15 & 23 & $5 d^{6}$ & $19096.89(6)$ & $152304.45(5)$ & pa & \\
\hline 503 & 1762 & 751.664 & 133038.13 & 751.6659 & 0.0011 & 0.30 & -0.002 & 14 & 26 & $5 d^{6}$ & $0.00(4)$ & $133037.83(3)$ & pa & \\
\hline 2996 & 1810 & 751.998 & 132978.99 & 751.9982 & 0.0022 & -0.06 & 0.000 & 5 & 12 & $5 d^{6}$ & $33303.67(6)$ & $166282.72(5)$ & pa & \\
\hline 53 & 232 & 752.318 & 132922.44 & 752.3179 & 0.0019 & -0.09 & 0.000 & 8 & 17 & $5 d^{6}$ & $26238.28(1)$ & $159160.81(2)$ & pa & \\
\hline 160 & 387 & 752.441 & 132900.84 & 752.4378 & 0.0011 & -0.52 & 0.003 & 9 & 37 & $5 d^{6}$ & $18463.42(5)$ & $151364.78(6)$ & pa & \\
\hline 716 & 1865 & 753.074 & 132789.09 & 753.0775 & 0.0011 & 0.64 & -0.004 & 8 & 39 & $5 d^{6}$ & $5824.11(3)$ & $138612.56(2)$ & pa & \\
\hline 97 & 400 & 753.543 & 132706.38 & 753.5471 & 0.0011 & 0.67 & -0.004 & 10 & 39 & $5 d^{6}$ & $5824.11(3)$ & $138529.81(3)$ & pa & \\
\hline 790 & 1305 & 753.564 & 132702.75 & 753.5639 & 0.0050 & 0.00 & 0.000 & 1 & 43 & $5 d^{6}$ & $28959.73(3)$ & $161662.48(2)$ & pa & \\
\hline 133 & 185 & 753.929 & 132638.43 & 753.9292 & 0.0012 & -0.02 & 0.000 & 12 & 39 & $5 d^{6}$ & $21190.03(3)$ & $153828.48(4)$ & pa & \\
\hline 1445 & 1870 & 754.034 & 132619.93 & 754.0366 & 0.0020 & 0.37 & -0.002 & 4 & 12 & $5 d^{6}$ & $33303.67(6)$ & $165923.23(6)$ & pa & \\
\hline 107 & 932 & 754.594 & 132521.58 & 754.5895 & 0.0012 & -0.80 & 0.005 & 12 & 30 & $5 d^{6}$ & $5178.26(2)$ & $137700.64(3)$ & pa & \\
\hline 256 & 525 & 755.232 & 132409.63 & 755.2359 & 0.0030 & 0.67 & -0.004 & 6 & 43 & $5 d^{6}$ & $28959.73(3)$ & $161368.69(3)$ & pa & \\
\hline 342 & 382 & 755.881 & 132295.92 & 755.8838 & 0.0018 & 0.46 & -0.003 & 9 & 23 & $5 d^{6}$ & $37387.78(4)$ & $169683.24(3)$ & pa & \\
\hline 346 & 1777 & 756.045 & 132267.33 & 756.0414 & 0.0013 & -0.56 & 0.003 & 9 & 23 & $5 d^{6}$ & $19096.89(6)$ & $151364.78(6)$ & pa & \\
\hline 749 & 1777 & 756.045 & 132267.33 & 756.0492 & 0.0014 & 0.80 & -0.005 & 11 & 25 & $5 d^{6}$ & $19128.73(2)$ & $151395.26(3)$ & pa & \\
\hline 168 & 1777 & 756.045 & 132267.33 & 756.0450 & 0.0013 & 0.07 & 0.000 & 7 & 36 & $5 d^{6}$ & $27940.65(4)$ & $160207.91(4)$ & pa & 300 \\
\hline 478 & 1290 & 756.185 & 132242.82 & 756.1858 & 0.0012 & 0.20 & -0.001 & 11 & 37 & $5 d^{6}$ & $18463.42(5)$ & $150706.05(4)$ & pa & \\
\hline 99 & 165 & 756.327 & 132217.96 & 756.3228 & 0.0012 & -0.72 & 0.004 & 12 & 30 & $5 d^{6}$ & $25969.65(4)$ & $158188.33(4)$ & pa & \\
\hline 63 & 200 & 756.986 & 132102.89 & 756.9837 & 0.0021 & -0.35 & 0.002 & 9 & 43 & $5 d^{6}$ & $28959.73(3)$ & $161062.97(2)$ & pa & 100 \\
\hline 217 & 200 & 756.986 & 132102.89 & 756.9867 & 0.0016 & 0.18 & -0.001 & 8 & 23 & $5 d^{6}$ & $37387.78(4)$ & $169490.49(5)$ & pa* & \\
\hline 51 & 1150 & 757.094 & 132083.98 & 757.0700 & 0.0011 & -4.20 & 0.024 & 15 & 26 & $5 d^{6}$ & $0.00(4)$ & $132088.18(4)$ & pa* & $300, \mathrm{bl}$ \\
\hline 20 & 95 & 757.277 & 132052.01 & 757.2843 & 0.0011 & 1.21 & -0.007 & 12 & 30 & $5 d^{6}$ & $5178.26(2)$ & $137229.06(2)$ & pa & \\
\hline 111 & 132 & 757.498 & 132013.50 & 757.4979 & 0.0015 & -0.07 & 0.000 & 13 & 25 & $5 d^{6}$ & $22138.98(2)$ & $154152.55(3)$ & pa & \\
\hline 597 & 1442 & 757.561 & 132002.50 & 757.5580 & 0.0017 & -0.60 & 0.003 & 9 & 39 & $5 d^{6}$ & $21190.03(3)$ & $153193.13(2)$ & pa & \\
\hline 58 & 57 & 757.628 & 131990.92 & 757.6228 & 0.0019 & -0.89 & 0.005 & 8 & 7 & $5 d^{6}$ & $8687.05(0)$ & $140678.86(1)$ & pa & \\
\hline 502 & 1630 & 757.881 & 131946.77 & 757.8820 & 0.0016 & 0.10 & -0.001 & 10 & 14 & $5 d^{6}$ & $8526.56(1)$ & $140473.23(2)$ & pa & \\
\hline 757 & 1947 & 758.282 & 131877.02 & 758.2850 & 0.0011 & 0.49 & -0.003 & 12 & 39 & $5 d^{6}$ & $5824.11(3)$ & $137700.64(3)$ & pa & \\
\hline 272 & 245 & 758.426 & 131851.95 & 758.4316 & 0.0019 & 0.90 & -0.005 & 8 & 36 & $5 d^{6}$ & $27940.65(4)$ & $159791.70(4)$ & pa & \\
\hline 835 & 1970 & 759.418 & 131679.77 & 759.4104 & 0.0017 & -1.34 & 0.008 & 8 & 31 & $5 d^{6}$ & $30693.33(3)$ & $162374.44(3)$ & pa $*$ & $\mathrm{bl}(\mathrm{O} \mathrm{V})$ \\
\hline 98 & 140 & 759.648 & 131639.95 & 759.6483 & 0.0013 & 0.09 & -0.001 & 17 & 32 & $5 d^{6}$ & $14546.32(4)$ & $146186.18(3)$ & pa & \\
\hline 502 & 1042 & 759.765 & 131619.65 & 759.7638 & 0.0020 & -0.20 & 0.001 & 2 & 25 & $5 d^{6}$ & $19128.73(2)$ & $150748.58(1)$ & pa & \\
\hline 684 & 557 & 760.132 & 131556.05 & 760.1334 & 0.0013 & 0.19 & -0.001 & 7 & 32 & $5 d^{6}$ & $28652.05(5)$ & $160207.91(4)$ & pa & \\
\hline 263 & 687 & 760.510 & 131490.64 & 760.5124 & 0.0018 & 0.35 & -0.002 & 8 & 30 & $5 d^{6}$ & $27670.52(2)$ & $159160.81(2)$ & pa & \\
\hline 2175 & 1552 & 760.725 & 131453.58 & 760.7267 & 0.0011 & 0.33 & -0.002 & 9 & 39 & $5 d^{6}$ & $5824.11(3)$ & $137277.36(4)$ & pa & \\
\hline 431 & 120 & 760.796 & 131441.19 & 760.7939 & 0.0030 & -0.44 & 0.003 & 5 & 31 & $5 d^{6}$ & $30693.33(3)$ & $162134.97(2)$ & pa & \\
\hline
\end{tabular}




\begin{tabular}{|c|c|c|c|c|c|c|c|c|c|c|c|c|c|c|}
\hline 608 & 977 & 761.001 & 131405.80 & 761.0063 & 0.0011 & 0.85 & -0.005 & 12 & 39 & $5 d^{6}$ & 5824.11(3) & $137229.06(2)$ & pa & \\
\hline 235 & 272 & 762.210 & 131197.37 & 762.2091 & 0.0015 & -0.23 & 0.001 & 11 & 30 & $5 d^{6}$ & $25969.65(4)$ & $157167.25(4)$ & pa & \\
\hline 31 & 12 & 762.299 & 131182.19 & 762.2904 & 0.0014 & -1.41 & 0.008 & 13 & 39 & $5 d^{6}$ & $21190.03(3)$ & $152373.63(3)$ & pa* & \\
\hline 559 & 1350 & 762.546 & 131139.63 & 762.5459 & 0.0019 & -0.02 & 0.000 & 8 & 32 & $5 d^{6}$ & $28652.05(5)$ & $159791.70(4)$ & pa & \\
\hline 485 & 1182 & 762.719 & 131109.95 & 762.7145 & 0.0015 & -0.71 & 0.004 & 8 & 32 & $5 d^{6}$ & $28652.05(5)$ & $159762.71(5)$ & pa & \\
\hline 180 & 260 & 763.040 & 131054.76 & 763.0434 & 0.0018 & 0.61 & -0.004 & 9 & 25 & $5 d^{6}$ & $22138.98(2)$ & $153193.13(2)$ & pa & \\
\hline 588 & 1485 & 763.415 & 130990.44 & 763.4149 & 0.0013 & 0.07 & 0.000 & 7 & 32 & $5 d^{6}$ & $14546.32(4)$ & $145536.69(3)$ & pa & \\
\hline 245 & 455 & 764.231 & 130850.42 & 764.2311 & 0.0030 & -0.05 & 0.000 & 7 & 8 & $5 d^{6}$ & $34466.53(1)$ & $165317.00(2)$ & pa & \\
\hline 277 & 467 & 764.345 & 130831.04 & 764.3392 & 0.0019 & -0.93 & 0.005 & 8 & 43 & $5 d^{6}$ & $28959.73(3)$ & $159791.70(4)$ & pa & \\
\hline 320 & 985 & 764.598 & 130787.68 & 764.5967 & 0.0013 & -0.23 & 0.001 & 11 & 30 & $5 d^{6}$ & $25969.65(4)$ & $156757.56(3)$ & pa & \\
\hline 39 & 107 & 764.691 & 130771.74 & 764.6886 & 0.0012 & -0.44 & 0.003 & 10 & 30 & $5 d^{6}$ & $5178.26(2)$ & $135950.44(1)$ & $\mathrm{pa}$ & \\
\hline 203 & 772 & 765.269 & 130672.95 & 765.2884 & 0.0022 & 3.26 & -0.019 & 6 & 43 & $5 d^{6}$ & $28959.73(3)$ & $159629.42(3)$ & pa* & * 400 \\
\hline 212 & 772 & 765.269 & 130672.95 & 765.2552 & 0.0041 & -2.40 & 0.014 & 6 & 31 & $5 d^{6}$ & $30693.33(3)$ & $161368.69(3)$ & pa* & 400 \\
\hline 298 & 1302 & 765.900 & 130565.37 & 765.9020 & 0.0016 & 0.36 & -0.002 & 5 & 23 & $5 d^{6}$ & $19096.89(6)$ & $149661.90(7)$ & pa & \\
\hline 656 & 1390 & 766.432 & 130474.79 & 766.4345 & 0.0013 & 0.49 & -0.003 & 12 & 37 & $5 d^{6}$ & $18463.42(5)$ & $148937.72(5)$ & pa & \\
\hline 676 & 1590 & 766.535 & 130457.20 & 766.5334 & 0.0010 & -0.26 & 0.002 & 19 & 32 & $5 d^{6}$ & $14546.32(4)$ & $145003.78(4)$ & pa & \\
\hline 344 & 495 & 767.042 & 130370.89 & 767.0498 & 0.0021 & 1.25 & -0.007 & 9 & 31 & $5 d^{6}$ & $30693.33(3)$ & $161062.97(2)$ & pa & \\
\hline 225 & 1457 & 767.422 & 130306.40 & 767.4226 & 0.0014 & 0.10 & -0.001 & 6 & 30 & $5 d^{6}$ & $5178.26(2)$ & $135484.56(1)$ & pa & \\
\hline 192 & 1457 & 767.422 & 130306.40 & 767.4077 & 0.0011 & -2.43 & 0.014 & 15 & 32 & $5 d^{6}$ & $14546.32(4)$ & $144855.15(3)$ & pa $*$ & * 300 \\
\hline 1000 & 1910 & 767.800 & 130242.32 & 767.8028 & 0.0011 & 0.55 & -0.003 & 15 & 30 & $5 d^{6}$ & $5178.26(2)$ & $135420.03(3)$ & pa & \\
\hline 1756 & 2012 & 767.947 & 130217.24 & 767.9531 & 0.0012 & 0.95 & -0.006 & 9 & 23 & $5 d^{6}$ & $19096.89(6)$ & $149313.18(6)$ & pa & \\
\hline 72 & 120 & 768.043 & 130200.99 & 768.0428 & 0.0018 & -0.09 & 0.001 & 8 & 43 & $5 d^{6}$ & $28959.73(3)$ & $159160.81(2)$ & pa & \\
\hline 55 & 62 & 768.106 & 130190.45 & 768.1026 & 0.0016 & -0.49 & 0.003 & 11 & 17 & $5 d^{6}$ & $26238.28(1)$ & $156429.22(2)$ & pa & \\
\hline 217 & 717 & 768.883 & 130058.87 & 768.8822 & 0.0020 & -0.07 & 0.000 & 2 & 39 & $5 d^{6}$ & $21190.03(3)$ & $151248.97(2)$ & pa & \\
\hline 137 & 1455 & 769.434 & 129965.66 & 769.4312 & 0.0010 & -0.47 & 0.003 & 14 & 32 & $5 d^{6}$ & $14546.32(4)$ & $144512.45(5)$ & pa & 700 \\
\hline 294 & 565 & 769.958 & 129877.21 & 769.9597 & 0.0021 & 0.29 & -0.002 & 7 & 29 & $5 d^{6}$ & $35440.07(3)$ & $165317.00(2)$ & pa & \\
\hline 428 & 1540 & 770.174 & 129840.84 & 770.1738 & 0.0014 & 0.01 & 0.000 & 12 & 23 & $5 d^{6}$ & $19096.89(6)$ & $148937.72(5)$ & pa & 1000 \\
\hline 8 & 35 & 770.589 & 129770.93 & 770.5862 & 0.0011 & -0.40 & 0.002 & 17 & 39 & $5 d^{6}$ & 5824.11(3) & $135595.44(4)$ & pa* & \\
\hline 221 & 230 & 770.809 & 129733.88 & 770.8131 & 0.0030 & 0.75 & -0.004 & 3 & 25 & $5 d^{6}$ & $22138.98(2)$ & $151872.11(1)$ & pa & \\
\hline 1146 & 1675 & 770.892 & 129719.86 & 770.8938 & 0.0016 & 0.31 & -0.002 & 7 & 23 & $5 d^{6}$ & $37387.78(4)$ & $167107.33(4)$ & pa & \\
\hline 1349 & 2000 & 771.433 & 129628.85 & 771.4367 & 0.0013 & 0.59 & -0.004 & 10 & 30 & $5 d^{6}$ & $25969.65(4)$ & $155597.91(4)$ & pa & \\
\hline 72 & 1082 & 771.635 & 129594.98 & 771.6292 & 0.0011 & -0.93 & 0.006 & 15 & 39 & $5 d^{6}$ & $5824.11(3)$ & $135420.03(3)$ & pa* & * 300 \\
\hline 99 & 220 & 771.859 & 129557.31 & 771.8643 & 0.0020 & 0.85 & -0.005 & 6 & 25 & $5 d^{6}$ & $19128.73(2)$ & $148685.19(2)$ & pa & Ir V \\
\hline 306 & 725 & 771.988 & 129535.74 & 771.9845 & 0.0012 & -0.54 & 0.003 & 12 & 32 & $5 d^{6}$ & $28652.05(5)$ & $158188.33(4)$ & pa & \\
\hline 84 & 280 & 772.026 & 129529.35 & 772.0256 & 0.0012 & -0.03 & 0.000 & 13 & 30 & $5 d^{6}$ & $5178.26(2)$ & $134707.64(2)$ & pa & \\
\hline 234 & 600 & 772.152 & 129508.18 & 772.1507 & 0.0020 & -0.23 & 0.001 & 4 & 25 & $5 d^{6}$ & $19128.73(2)$ & $148637.14(1)$ & pa & \\
\hline 250 & 412 & 772.261 & 129489.90 & 772.2691 & 0.0023 & 1.35 & -0.008 & 3 & 23 & $5 d^{6}$ & $37387.78(4)$ & $166876.33(4)$ & pa & \\
\hline 109 & 192 & 772.392 & 129468.02 & 772.3807 & 0.0023 & -1.81 & 0.011 & 7 & 29 & $5 d^{6}$ & $35440.07(3)$ & $164909.90(2)$ & pa $*$ & \\
\hline 421 & 1290 & 772.665 & 129422.23 & 772.6706 & 0.0012 & 0.97 & -0.006 & 11 & 37 & $5 d^{6}$ & $18463.42(5)$ & $147884.68(4)$ & pa & \\
\hline 2249 & 1505 & 773.090 & 129350.98 & 773.0946 & 0.0017 & 0.71 & -0.004 & 11 & 12 & $5 d^{6}$ & $33303.67(6)$ & $162653.94(5)$ & pa & \\
\hline 1947 & 1572 & 773.394 & 129300.13 & 773.3964 & 0.0012 & 0.34 & -0.002 & 11 & 37 & $5 d^{6}$ & $18463.42(5)$ & $147763.22(4)$ & pa & \\
\hline 363 & 1072 & 773.819 & 129229.17 & 773.8225 & 0.0012 & 0.57 & -0.003 & 12 & 43 & $5 d^{6}$ & $28959.73(3)$ & $158188.33(4)$ & pa & \\
\hline 237 & 755 & 774.042 & 129191.94 & 774.0480 & 0.0013 & 0.98 & -0.006 & 12 & 30 & $5 d^{6}$ & $25969.65(4)$ & $155160.61(5)$ & pa & \\
\hline 152 & 1895 & 774.518 & 129112.59 & 774.5011 & 0.0016 & -2.78 & 0.017 & 13 & 30 & $5 d^{6}$ & $25969.65(4)$ & $155085.02(3)$ & pa* & * 200, \\
\hline
\end{tabular}




\begin{tabular}{|c|c|c|c|c|c|c|c|c|c|c|c|c|c|}
\hline 97 & 112 & 774.668 & 129087.57 & 774.6711 & 0.0012 & 0.53 & -0.003 & 11 & 30 & $5 d^{6}$ & $27670.52(2)$ & $156757.56(3)$ & pa \\
\hline 997 & 2070 & 774.773 & 129070.06 & 774.7735 & 0.0012 & 0.08 & 0.000 & 10 & 26 & $5 d^{6}$ & $0.00(4)$ & $129069.98(3)$ & pa \\
\hline 353 & 587 & 774.924 & 129044.85 & 774.9231 & 0.0013 & -0.21 & 0.001 & 11 & 39 & $5 d^{6}$ & $21190.03(3)$ & $150235.09(3)$ & pa \\
\hline 786 & 1835 & 775.150 & 129007.34 & 775.1519 & 0.0015 & 0.37 & -0.002 & 8 & 25 & $5 d^{6}$ & $19128.73(2)$ & $148135.70(2)$ & pa \\
\hline 710 & 1292 & 775.571 & 128937.23 & 775.5781 & 0.0022 & 1.14 & -0.007 & 6 & 31 & $5 d^{6}$ & $30693.33(3)$ & $159629.42(3)$ & pa \\
\hline 217 & 1090 & 775.899 & 128882.79 & 775.8943 & 0.0012 & -0.74 & 0.004 & 13 & 39 & $5 d^{6}$ & 5824.11(3) & $134707.64(2)$ & pa \\
\hline 522 & 1010 & 776.225 & 128828.64 & 776.2227 & 0.0012 & -0.36 & 0.002 & 12 & 32 & $5 d^{6}$ & $28652.05(5)$ & $157481.05(5)$ & pa \\
\hline 521 & 95 & 776.293 & 128817.27 & 776.2956 & 0.0012 & 0.36 & -0.002 & 11 & 36 & $5 d^{6}$ & $27940.65(4)$ & $156757.56(3)$ & pa \\
\hline 47 & 167 & 776.987 & 128702.23 & 776.9857 & 0.0014 & -0.27 & 0.002 & 12 & 14 & $5 d^{6}$ & $8526.56(1)$ & $137229.06(2)$ & pa \\
\hline 30 & 200 & 777.141 & 128676.84 & 777.1473 & 0.0013 & 1.10 & -0.007 & 11 & 32 & $5 d^{6}$ & $28652.05(5)$ & $157327.79(6)$ & pa $*$ \\
\hline 175 & 217 & 777.166 & 128672.59 & 777.1647 & 0.0018 & -0.27 & 0.002 & 4 & 20 & $5 d^{6}$ & $46354.20(4)$ & $175027.06(4)$ & pa \\
\hline 396 & 450 & 777.302 & 128650.18 & 777.3017 & 0.0050 & 0.00 & 0.000 & 1 & 8 & $5 d^{6}$ & $34466.53(1)$ & $163116.70(1)$ & pa \\
\hline 574 & 1575 & 777.514 & 128615.11 & 777.5076 & 0.0016 & -1.00 & 0.006 & 8 & 14 & $5 d^{6}$ & $8526.56(1)$ & $137142.67(1)$ & pa \\
\hline 189 & 1575 & 777.514 & 128615.11 & 777.5217 & 0.0030 & 1.33 & -0.008 & 12 & 17 & $5 d^{6}$ & $26238.28(1)$ & $154852.06(2)$ & pa $* 200$ \\
\hline 273 & 275 & 777.872 & 128555.84 & 777.8646 & 0.0015 & -1.23 & 0.007 & 8 & 29 & $5 d^{6}$ & $35440.07(3)$ & $163997.14(4)$ & pa \\
\hline 1387 & 1527 & 778.118 & 128515.16 & 778.1181 & 0.0015 & -0.04 & 0.000 & 11 & 32 & $5 d^{6}$ & $28652.05(5)$ & $157167.25(4)$ & pa \\
\hline 146 & 1552 & 778.242 & 128494.74 & 778.2512 & 0.0011 & 1.53 & -0.009 & 14 & 26 & $5 d^{6}$ & $0.00(4)$ & $128493.21(4)$ & pa \\
\hline 236 & 1370 & 778.477 & 128455.93 & 778.4790 & 0.0020 & 0.31 & -0.002 & 8 & 7 & $5 d^{6}$ & $8687.05(0)$ & $137142.67(1)$ & pa \\
\hline 315 & 287 & 779.450 & 128295.58 & 779.4540 & 0.0022 & 0.65 & -0.004 & 8 & 29 & $5 d^{6}$ & $35440.07(3)$ & $163735.00(3)$ & pa \\
\hline 176 & 500 & 779.953 & 128212.94 & 779.9521 & 0.0014 & -0.07 & 0.000 & 10 & 25 & $5 d^{6}$ & $19128.73(2)$ & $147341.74(3)$ & pa \\
\hline 93 & 310 & 780.029 & 128200.33 & 780.0239 & 0.0010 & -0.87 & 0.005 & 16 & 39 & $5 d^{6}$ & $5824.11(3)$ & $134025.31(4)$ & pa \\
\hline 950 & 1532 & 780.134 & 128183.14 & 780.1353 & 0.0014 & 0.24 & -0.001 & 13 & 30 & $5 d^{6}$ & $25969.65(4)$ & $154152.55(3)$ & pa \\
\hline 148 & 100 & 780.394 & 128140.47 & 780.3973 & 0.0021 & 0.61 & -0.004 & 5 & 43 & $5 d^{6}$ & $28959.73(3)$ & $157099.59(2)$ & pa \\
\hline 100 & 377 & 780.671 & 128094.87 & 780.6638 & 0.0015 & -1.24 & 0.008 & 11 & 25 & $5 d^{6}$ & $22138.98(2)$ & $150235.09(3)$ & pa \\
\hline 64 & 80 & 780.799 & 128073.87 & 780.7956 & 0.0016 & -0.63 & 0.004 & 8 & 39 & $5 d^{6}$ & $21190.03(3)$ & $149264.53(2)$ & pa \\
\hline 194 & 1187 & 781.093 & 128025.68 & 781.0923 & 0.0013 & -0.16 & 0.001 & 11 & 30 & $5 d^{6}$ & $5178.26(2)$ & $133204.10(2)$ & pa \\
\hline 570 & 2057 & 782.108 & 127859.65 & 782.1081 & 0.0011 & 0.08 & 0.000 & 14 & 30 & $5 d^{6}$ & $5178.26(2)$ & $133037.83(3)$ & pa \\
\hline 701 & 2057 & 782.108 & 127859.65 & 782.1126 & 0.0014 & 0.81 & -0.005 & 12 & 30 & $5 d^{6}$ & $25969.65(4)$ & $153828.48(4)$ & pa \\
\hline 82 & 285 & 783.151 & 127689.21 & 783.1508 & 0.0013 & -0.12 & 0.001 & 12 & 30 & $5 d^{6}$ & $25969.65(4)$ & $153658.98(5)$ & pa \\
\hline 1263 & 345 & 783.192 & 127682.63 & 783.1964 & 0.0031 & 0.73 & -0.004 & 3 & 16 & $5 d^{6}$ & $52259.88(3)$ & $179941.78(3)$ & pa \\
\hline 597 & 1672 & 783.347 & 127657.27 & 783.3475 & 0.0013 & 0.01 & 0.000 & 10 & 36 & $5 d^{6}$ & $27940.65(4)$ & $155597.91(4)$ & pa \\
\hline 899 & 1840 & 783.376 & 127652.65 & 783.3770 & 0.0011 & 0.19 & -0.001 & 13 & 32 & $5 d^{6}$ & $14546.32(4)$ & $142198.78(4)$ & pa \\
\hline 95 & 382 & 784.343 & 127495.19 & 784.3435 & 0.0020 & 0.03 & 0.000 & 6 & 39 & $5 d^{6}$ & $21190.03(3)$ & $148685.19(2)$ & pa \\
\hline 121 & 462 & 784.503 & 127469.27 & 784.5014 & 0.0014 & -0.22 & 0.001 & 11 & 43 & $5 d^{6}$ & $28959.73(3)$ & $156429.22(2)$ & pa \\
\hline 381 & 1612 & 784.783 & 127423.84 & 784.7823 & 0.0016 & -0.04 & 0.000 & 10 & 14 & $5 d^{6}$ & $8526.56(1)$ & $135950.44(1)$ & pa \\
\hline 127 & 152 & 784.843 & 127414.07 & 784.8400 & 0.0016 & -0.43 & 0.003 & 13 & 30 & $5 d^{6}$ & $27670.52(2)$ & $155085.02(3)$ & pa \\
\hline 70 & 247 & 785.054 & 127379.82 & 785.0527 & 0.0013 & -0.17 & 0.001 & 11 & 39 & $5 d^{6}$ & $5824.11(3)$ & $133204.10(2)$ & pa \\
\hline 702 & 967 & 785.202 & 127355.81 & 785.2008 & 0.0018 & -0.14 & 0.001 & 8 & 23 & $5 d^{6}$ & $37387.78(4)$ & $164743.73(4)$ & pa \\
\hline 312 & 1332 & 785.605 & 127290.37 & 785.6074 & 0.0012 & 0.32 & -0.002 & 8 & 30 & $5 d^{6}$ & $5178.26(2)$ & $132468.31(2)$ & pa \\
\hline 135 & 212 & 785.769 & 127263.83 & 785.7719 & 0.0019 & 0.44 & -0.003 & 10 & 7 & $5 d^{6}$ & $8687.05(0)$ & $135950.44(1)$ & pa \\
\hline 421 & 1647 & 786.077 & 127214.05 & 786.0787 & 0.0011 & 0.33 & -0.002 & 14 & 39 & $5 d^{6}$ & $5824.11(3)$ & $133037.83(3)$ & pa \\
\hline 148 & 150 & 786.267 & 127183.20 & 786.2776 & 0.0031 & 1.66 & -0.010 & 12 & 30 & $5 d^{6}$ & $27670.52(2)$ & $154852.06(2)$ & pa \\
\hline 470 & 885 & 786.357 & 127168.65 & 786.3567 & 0.0014 & -0.10 & 0.001 & 8 & 39 & $5 d^{6}$ & $21190.03(3)$ & $148358.78(3)$ & pa \\
\hline 495 & 1327 & 786.619 & 127126.36 & 786.6239 & 0.0018 & 0.81 & -0.005 & 8 & 25 & $5 d^{6}$ & $22138.98(2)$ & $149264.53(2)$ & pa \\
\hline
\end{tabular}




\begin{tabular}{|c|c|c|c|c|c|c|c|c|c|c|c|c|c|c|}
\hline 769 & 1637 & 787.019 & 127061.77 & 787.0184 & 0.0011 & -0.06 & 0.000 & 17 & 37 & $5 d^{6}$ & $18463.42(5)$ & $145525.25(5)$ & pa & \\
\hline 115 & 397 & 787.540 & 126977.66 & 787.5471 & 0.0011 & 1.13 & -0.007 & 16 & 37 & $5 d^{6}$ & $18463.42(5)$ & $145439.95(4)$ & pa & \\
\hline 293 & 310 & 787.801 & 126935.58 & 787.8087 & 0.0018 & 1.21 & -0.007 & 8 & 29 & $5 d^{6}$ & $35440.07(3)$ & $162374.44(3)$ & pa & \\
\hline 325 & 1425 & 788.657 & 126797.80 & 788.6590 & 0.0021 & 0.29 & -0.002 & 6 & 7 & $5 d^{6}$ & $8687.05(0)$ & $135484.56(1)$ & pa & \\
\hline 667 & 1732 & 789.294 & 126695.54 & 789.2993 & 0.0013 & 0.89 & -0.006 & 11 & 39 & $5 d^{6}$ & $21190.03(3)$ & $147884.68(4)$ & pa & \\
\hline 130 & 1732 & 789.294 & 126695.54 & 789.2978 & 0.0030 & 0.64 & -0.004 & 5 & 29 & $5 d^{6}$ & $35440.07(3)$ & $162134.97(2)$ & pa* & 200 \\
\hline 1481 & 2100 & 789.462 & 126668.59 & 789.4651 & 0.0012 & 0.55 & -0.003 & 14 & 32 & $5 d^{6}$ & $14546.32(4)$ & $141214.36(3)$ & pa & \\
\hline 107 & 82 & 789.651 & 126638.22 & 789.6513 & 0.0013 & 0.04 & 0.000 & 10 & 43 & $5 d^{6}$ & $28959.73(3)$ & $155597.91(4)$ & pa & \\
\hline 620 & 985 & 789.829 & 126609.70 & 789.8310 & 0.0017 & 0.34 & -0.002 & 8 & 23 & $5 d^{6}$ & $37387.78(4)$ & $163997.14(4)$ & pa & \\
\hline 136 & 342 & 789.915 & 126595.85 & 789.9216 & 0.0012 & 1.01 & -0.006 & 15 & 32 & $5 d^{6}$ & $14546.32(4)$ & $141141.16(4)$ & pa & \\
\hline 226 & 342 & 789.915 & 126595.85 & 789.9116 & 0.0030 & -0.59 & 0.004 & 9 & 8 & $5 d^{6}$ & $34466.53(1)$ & $161062.97(2)$ & pa & \\
\hline 276 & 1737 & 790.202 & 126550.00 & 790.2252 & 0.0022 & 3.79 & -0.024 & 6 & 25 & $5 d^{6}$ & $22138.98(2)$ & $148685.19(2)$ & pa* & $600, \mathrm{bl}(\mathrm{O}$ IV $)$ \\
\hline 1048 & 1242 & 790.259 & 126540.86 & 790.2617 & 0.0010 & 0.50 & -0.003 & 19 & 37 & $5 d^{6}$ & $18463.42(5)$ & $145003.78(4)$ & pa & \\
\hline 1894 & 1525 & 790.460 & 126508.66 & 790.4603 & 0.0013 & 0.10 & -0.001 & 12 & 32 & $5 d^{6}$ & $28652.05(5)$ & $155160.61(5)$ & pa & \\
\hline 219 & 210 & 790.525 & 126498.16 & 790.5254 & 0.0021 & 0.01 & 0.000 & 4 & 25 & $5 d^{6}$ & $22138.98(2)$ & $148637.14(1)$ & pa & \\
\hline 297 & 365 & 790.677 & 126473.98 & 790.6769 & 0.0016 & 0.06 & 0.000 & 11 & 31 & $5 d^{6}$ & $30693.33(3)$ & $157167.25(4)$ & pa & \\
\hline 43 & 25 & 790.752 & 126461.87 & 790.7517 & 0.0016 & -0.07 & 0.000 & 9 & 29 & $5 d^{6}$ & $35440.07(3)$ & $161902.01(4)$ & pa* & \\
\hline 2386 & 2117 & 790.960 & 126428.63 & 790.9618 & 0.0013 & 0.27 & -0.002 & 17 & 23 & $5 d^{6}$ & $19096.89(6)$ & $145525.25(5)$ & pa & \\
\hline 94 & 175 & 791.099 & 126406.41 & 791.1001 & 0.0031 & 0.16 & -0.001 & 5 & 31 & $5 d^{6}$ & $30693.33(3)$ & $157099.59(2)$ & pa & \\
\hline 222 & 165 & 791.450 & 126350.42 & 791.4697 & 0.0024 & 3.20 & -0.020 & 8 & 23 & $5 d^{6}$ & $37387.78(4)$ & $163735.00(3)$ & pa* & 100 \\
\hline 817 & 1545 & 791.545 & 126335.19 & 791.5475 & 0.0012 & 0.39 & -0.002 & 15 & 30 & $5 d^{6}$ & $25969.65(4)$ & $152304.45(5)$ & pa & \\
\hline 44 & 150 & 791.991 & 126264.03 & 791.9909 & 0.0011 & -0.04 & 0.000 & 15 & 39 & $5 d^{6}$ & $5824.11(3)$ & $132088.18(4)$ & pa & \\
\hline 487 & 947 & 792.318 & 126211.97 & 792.3183 & 0.0015 & 0.07 & 0.000 & 13 & 36 & $5 d^{6}$ & $27940.65(4)$ & $154152.55(3)$ & pa & \\
\hline 301 & 1067 & 792.510 & 126181.34 & 792.5118 & 0.0015 & 0.26 & -0.002 & 13 & 14 & $5 d^{6}$ & $8526.56(1)$ & $134707.64(2)$ & pa & \\
\hline 746 & 780 & 792.695 & 126152.00 & 792.6963 & 0.0014 & 0.29 & -0.002 & 10 & 39 & $5 d^{6}$ & $21190.03(3)$ & $147341.74(3)$ & pa & \\
\hline 255 & 125 & 792.860 & 126125.64 & 792.8624 & 0.0016 & 0.35 & -0.002 & 13 & 43 & $5 d^{6}$ & $28959.73(3)$ & $155085.02(3)$ & pa & \\
\hline 1364 & 1302 & 792.942 & 126112.69 & 792.9422 & 0.0020 & 0.09 & -0.001 & 4 & 12 & $5 d^{6}$ & $33303.67(6)$ & $159416.27(6)$ & pa & \\
\hline 892 & 952 & 793.265 & 126061.34 & 793.2680 & 0.0022 & 0.54 & -0.003 & 2 & 30 & $5 d^{6}$ & $27670.52(2)$ & $153731.32(1)$ & pa & \\
\hline 830 & 1290 & 793.346 & 126048.46 & 793.3421 & 0.0011 & -0.57 & 0.004 & 14 & 37 & $5 d^{6}$ & $18463.42(5)$ & $144512.45(5)$ & pa & \\
\hline 2194 & 2135 & 793.761 & 125982.59 & 793.7633 & 0.0012 & 0.45 & -0.003 & 11 & 26 & $5 d^{6}$ & $0.00(4)$ & $125982.14(5)$ & pa & \\
\hline 1643 & 720 & 793.903 & 125959.94 & 793.9042 & 0.0031 & 0.16 & -0.001 & 3 & 10 & $5 d^{6}$ & $53982.00(4)$ & $179941.78(3)$ & pa & \\
\hline 437 & 1167 & 794.350 & 125889.04 & 794.3580 & 0.0013 & 1.21 & -0.008 & 12 & 36 & $5 d^{6}$ & $27940.65(4)$ & $153828.48(4)$ & pa & \\
\hline 213 & 1167 & 794.350 & 125889.04 & 794.3296 & 0.0031 & -3.29 & 0.021 & 12 & 43 & $5 d^{6}$ & $28959.73(3)$ & $154852.06(2)$ & pa* & 200 \\
\hline 72 & 35 & 794.577 & 125853.05 & 794.5780 & 0.0018 & 0.08 & 0.000 & 7 & 23 & $5 d^{6}$ & $37387.78(4)$ & $163240.75(5)$ & pa* & \\
\hline 73 & 85 & 794.792 & 125819.16 & 794.7999 & 0.0019 & 1.33 & -0.008 & 8 & 23 & $5 d^{6}$ & $37387.78(4)$ & $163205.61(3)$ & pa* & \\
\hline 167 & 87 & 794.983 & 125788.88 & 794.9794 & 0.0030 & -0.53 & 0.003 & 7 & 7 & $5 d^{6}$ & $39120.48(2)$ & $164909.90(2)$ & pa & \\
\hline 348 & 455 & 795.316 & 125736.12 & 795.3179 & 0.0015 & 0.24 & -0.001 & 11 & 31 & $5 d^{6}$ & $30693.33(3)$ & $156429.22(2)$ & pa & \\
\hline 190 & 367 & 795.371 & 125727.54 & 795.3778 & 0.0013 & 1.12 & -0.007 & 15 & 25 & $5 d^{6}$ & $19128.73(2)$ & $144855.15(3)$ & pa & \\
\hline 84 & 102 & 795.439 & 125716.71 & 795.4290 & 0.0012 & -1.62 & 0.010 & 12 & 36 & $5 d^{6}$ & $27940.65(4)$ & $153658.98(5)$ & pa* & \\
\hline 847 & 1967 & 795.564 & 125696.97 & 795.5641 & 0.0012 & -0.01 & 0.000 & 10 & 30 & $5 d^{6}$ & $5178.26(2)$ & $130875.24(2)$ & pa & \\
\hline 1193 & 1600 & 795.917 & 125641.27 & 795.9143 & 0.0012 & -0.39 & 0.002 & 7 & 32 & $5 d^{6}$ & $28652.05(5)$ & $154293.71(6)$ & pa & \\
\hline 118 & 127 & 795.971 & 125632.78 & 795.9640 & 0.0030 & -1.05 & 0.007 & 3 & 17 & $5 d^{6}$ & $26238.28(1)$ & $151872.11(1)$ & pa & \\
\hline 50 & 320 & 796.664 & 125523.45 & 796.6692 & 0.0019 & 0.84 & -0.005 & 9 & 30 & $5 d^{6}$ & $27670.52(2)$ & $153193.13(2)$ & pa & O II \\
\hline 631 & 1605 & 796.828 & 125497.66 & 796.8291 & 0.0014 & 0.24 & -0.002 & 9 & 25 & $5 d^{6}$ & $19128.73(2)$ & $144626.15(2)$ & pa & \\
\hline
\end{tabular}




$\begin{array}{rrrr}153 & 452 & 796.994 & 125471.51 \\ 86 & 212 & 797.118 & 125451.86 \\ 69 & 232 & 797.345 & 125416.27 \\ 174 & 1467 & 798.293 & 125267.21 \\ 430 & 1467 & 798.293 & 125267.21 \\ 866 & 2030 & 798.564 & 125224.83 \\ 194 & 200 & 798.703 & 125202.97 \\ 615 & 1670 & 798.762 & 125193.77 \\ 179 & 1670 & 798.762 & 125193.77 \\ 165 & 245 & 798.870 & 125176.84 \\ 86 & 310 & 799.091 & 125142.19 \\ 74 & 280 & 799.668 & 125051.90 \\ 953 & 1387 & 799.730 & 125042.14 \\ 302 & 352 & 799.807 & 125030.21 \\ 918 & 1650 & 799.951 & 125007.58 \\ 39 & 80 & 800.032 & 124994.97 \\ 360 & 795 & 800.839 & 124869.11 \\ 22 & 15 & 801.527 & 124761.88 \\ 155 & 277 & 801.910 & 124702.27 \\ 39 & 397 & 801.953 & 124695.52 \\ 209 & 397 & 801.953 & 124695.52 \\ 197 & 1272 & 802.070 & 124677.35 \\ 280 & 1375 & 802.342 & 124635.18 \\ 255 & 422 & 803.132 & 124512.53 \\ 399 & 1110 & 803.645 & 124433.07 \\ 299 & 967 & 803.911 & 124391.90 \\ 83 & 105 & 804.094 & 124363.55 \\ 72 & 65 & 804.180 & 124350.25 \\ 652 & 1802 & 804.736 & 124264.43 \\ 1027 & 1802 & 804.736 & 124264.43 \\ 107 & 340 & 804.831 & 124249.72 \\ 393 & 650 & 805.159 & 124199.10 \\ 333 & 342 & 805.208 & 124191.50 \\ 477 & 275 & 805.297 & 124177.71 \\ 863 & 992 & 805.356 & 124168.66 \\ 353 & 442 & 805.427 & 124157.73 \\ 239 & 130 & 805.892 & 124086.03 \\ 539 & 1252 & 806.144 & 124047.24 \\ 3209 & 1892 & 806.295 & 124024.15 \\ 135 & 775 & 806.560 & 123983.31 \\ 24 & 315 & 806.835 & 123941.09 \\ 39 & 167 & 807.151 & 123892.57 \\ 273 & 747 & 808.244 & 123725.03 \\ 179 & 312 & 808.720 & 123652.21\end{array}$

$\begin{array}{lll}796.9939 & 0.0012 & 0.03\end{array}$ $\begin{array}{lll}797.1172 & 0.0011 & -0.20\end{array}$ $\begin{array}{lll}797.3492 & 0.0013 & 0.71\end{array}$ $\begin{array}{llll}798.2858 & 0.0012 & -1.22\end{array}$ $\begin{array}{lll}798.3002 & 0.0016 & 1.05\end{array}$ $\begin{array}{llll}798.5638 & 0.0012 & 0.02\end{array}$ $\begin{array}{lll}798.7044 & 0.0016 & 0.21\end{array}$ $\begin{array}{llll}798.7636 & 0.0011 & 0.28\end{array}$ $\begin{array}{lll}798.7679 & 0.0015 & 0.95\end{array}$ $\begin{array}{lll}798.8724 & 0.0014 & 0.41\end{array}$ $\begin{array}{lll}799.1026 & 0.0015 & 1.81\end{array}$ $\begin{array}{lll}799.6729 & 0.0012 & 0.77\end{array}$ $\begin{array}{lll}799.7337 & 0.0013 & 0.52\end{array}$ $\begin{array}{lll}799.8049 & 0.0014 & -0.28\end{array}$ $\begin{array}{lll}799.9557 & 0.0013 & 0.65\end{array}$ $\begin{array}{llll}800.0246 & 0.0014 & -1.18\end{array}$ $\begin{array}{llll}800.8409 & 0.0014 & 0.36\end{array}$ $\begin{array}{lll}801.5384 & 0.0013 & 1.80\end{array}$ $\begin{array}{llll}801.9046 & 0.0015 & -0.84\end{array}$ $\begin{array}{lll}801.9663 & 0.0011 & 2.00\end{array}$ $\begin{array}{lll}801.9614 & 0.0034 & 1.24\end{array}$ $\begin{array}{lll}802.0691 & 0.0016 & -0.19\end{array}$ $\begin{array}{lll}802.3431 & 0.0013 & 0.22\end{array}$ $\begin{array}{llll}803.1211 & 0.0017 & -1.70\end{array}$ $\begin{array}{lll}803.6455 & 0.0016 & 0.09\end{array}$ $\begin{array}{llll}803.9123 & 0.0017 & 0.21\end{array}$ $\begin{array}{llll}804.0925 & 0.0012 & -0.25\end{array}$ $\begin{array}{llll}804.1712 & 0.0023 & -1.38\end{array}$ $\begin{array}{lll}804.7290 & 0.0015 & -1.01\end{array}$ $\begin{array}{lll}804.7362 & 0.0018 & 0.10\end{array}$ $\begin{array}{llll}804.8295 & 0.0011 & -0.20\end{array}$ $\begin{array}{lll}805.1608 & 0.0012 & 0.31\end{array}$ $\begin{array}{llll}805.2085 & 0.0017 & 0.07\end{array}$ $\begin{array}{llll}805.2996 & 0.0018 & 0.33\end{array}$ $\begin{array}{llll}805.3539 & 0.0014 & -0.36\end{array}$ $\begin{array}{llll}805.4206 & 0.0031 & -1.00\end{array}$ $\begin{array}{lll}805.8983 & 0.0030 & 0.90\end{array}$ $\begin{array}{llll}806.1448 & 0.0015 & 0.04\end{array}$ $\begin{array}{lll}806.2948 & 0.0020 & 0.03\end{array}$ $\begin{array}{llll}806.5590 & 0.0013 & -0.18\end{array}$ $\begin{array}{llll}806.8306 & 0.0016 & -0.66\end{array}$ $\begin{array}{llll}807.1564 & 0.0012 & 0.85\end{array}$ $\begin{array}{lll}808.2458 & 0.0013 & 0.29\end{array}$ $\begin{array}{llll}808.7186 & 0.0012 & -0.19\end{array}$ $\begin{array}{llll}0.000 & 14 & 36 & 5 \mathrm{~d}^{6}\end{array}$ $\begin{array}{llll}0.001 & 15 & 32 & 5 \mathrm{~d}^{6}\end{array}$

$\begin{array}{llll}-0.005 & 14 & 23 & 5 \mathrm{~d}^{6}\end{array}$

$\begin{array}{llll}0.008 & 14 & 37 & 5 \mathrm{~d}^{6}\end{array}$

$\begin{array}{llll}-0.007 & 11 & 23 & 5 d^{6}\end{array}$

$\begin{array}{llll}0.000 & 15 & 26 & 5 \mathrm{~d}^{6}\end{array}$

$\begin{array}{llll}-0.001 & 10 & 25 & 5 d^{6}\end{array}$

$\begin{array}{llll}-0.002 & 16 & 32 & 5 d^{6}\end{array}$

$\begin{array}{llll}-0.006 & 13 & 43 & 5 d^{6}\end{array}$

$\begin{array}{llll}-0.003 & 12 & 32 & 5 d^{6}\end{array}$

$\begin{array}{llll}-0.012 & 6 & 39 & 5 d^{6}\end{array}$

$\begin{array}{llll}-0.005 & 10 & 39 & 5 d^{6}\end{array}$

$\begin{array}{llll}-0.003 & 8 & 36 & 5 d^{6}\end{array}$

$\begin{array}{llll}0.002 & 10 & 30 & 5 d^{6}\end{array}$

$\begin{array}{llll}-0.004 & 12 & 32 & 5 d^{6}\end{array}$

$\begin{array}{llll}0.008 & 17 & 39 & 5 \mathrm{~d}^{6}\end{array}$

$\begin{array}{llll}-0.002 & 12 & 43 & 5 d^{6}\end{array}$

$\begin{array}{llll}-0.012 & 14 & 32 & 5 d^{6}\end{array}$

$\begin{array}{llll}0.005 & 13 & 30 & 5 \mathrm{~d}^{6}\end{array}$

$\begin{array}{llll}-0.013 & 16 & 37 & 5 d^{6}\end{array}$

$\begin{array}{llll}-0.008 & 8 & 8 & 5 d^{6}\end{array}$

$\begin{array}{llll}0.001 & 11 & 14 & 5 d^{6}\end{array}$

$\begin{array}{llll}-0.001 & 14 & 23 & 5 \mathrm{~d}^{6}\end{array}$

$\begin{array}{llll}0.011 & 9 & 23 & 5 \mathrm{~d}^{6}\end{array}$

$\begin{array}{llll}-0.001 & 13 & 36 & 5 d^{6}\end{array}$

$\begin{array}{llll}-0.001 & 13 & 31 & 5 \mathrm{~d}^{6}\end{array}$

$\begin{array}{llll}0.002 & 15 & 36 & 5 d^{6}\end{array}$

$\begin{array}{llll}0.009 & 8 & 29 & 5 \mathrm{~d}^{6}\end{array}$

$\begin{array}{llll}0.007 & 11 & 30 & 5 d^{6}\end{array}$

$\begin{array}{llll}-0.001 & 6 & 20 & 5 \mathrm{~d}^{6}\end{array}$

$\begin{array}{llll}0.001 & 16 & 39 & 5 \mathrm{~d}^{6}\end{array}$

$\begin{array}{llll}-0.002 & 10 & 37 & 5 d^{6}\end{array}$

$\begin{array}{llll}0.000 & 6 & 25 & 5 \mathrm{~d}^{6}\end{array}$

$\begin{array}{llll}-0.002 & 12 & 12 & 5 d^{6} \\ 0\end{array}$

$\begin{array}{llll}0.002 & 9 & 39 & 5 \mathrm{~d}^{6}\end{array}$

$\begin{array}{llll}0.006 & 12 & 31 & 5 \mathrm{~d}^{6}\end{array}$

$\begin{array}{llll}-0.006 & 8 & 7 & 5 d^{6}\end{array}$

$\begin{array}{llll}0.000 & 17 & 25 & 5 \mathrm{~d}^{6}\end{array}$

$\begin{array}{llll}0.000 & 11 & 12 & 5 d^{6}\end{array}$

$\begin{array}{llll}0.001 & 10 & 32 & 5 \mathrm{~d}^{6}\end{array}$

$\begin{array}{llll}0.004 & 8 & 14 & 5 d^{6}\end{array}$

$\begin{array}{llll}-0.006 & 10 & 30 & 5 \mathrm{~d}^{6}\end{array}$

$\begin{array}{llll}-0.002 & 11 & 30 & 5 d^{6}\end{array}$

$\begin{array}{llll}0.001 & 15 & 32 & 5 d^{6}\end{array}$
$27940.65(4) \quad 153412.13(4) \quad$ pa

$\begin{array}{lll}14546.32(4) & 139998.38(4) \quad \text { pa }\end{array}$

$19096.89(6) \quad 144512.45(5) \quad$ pa

$18463.42(5) \quad 143731.85(5) \quad$ pa $* 1000$

$37387.78(4) \quad 162653.94(5)$ pa 1000

$0.00(4) \quad 125224.81(4) \quad$ pa

$22138.98(2) \quad 147341.74(3) \quad$ pa

$14546.32(4) \quad 139739.81(5) \quad$ pa

$28959.73(3) \quad 154152.55(3)$ pa 300

$28652.05(5) \quad 153828.48(4) \quad$ pa

21190.03(3) 146330.41(2) pa

5824.11(3) 130875.24(2) pa C II

27940.65(4) 152982.27(4) pa

$25969.65(4) \quad 151000.14(5) \quad$ pa

$28652.05(5) \quad 153658.98(5) \quad$ pa

$21190.03(3) \quad 146186.18(3) \quad$ pa

28959.73(3) $153828.48(4) \quad$ pa

$28652.05(5) \quad 153412.13(4) \quad$ pa *

$27670.52(2) \quad 152373.63(3) \quad \mathrm{pa}$

$18463.42(5) \quad 143156.95(4) \quad$ pa $* 100$

$34466.53(1) \quad 159160.81(2) \quad$ pa

8526.56(1) 133204.10(2) pa

19096.89(6) $143731.85(5) \quad$ pa

37387.78(4) 161902.01(4) pa

27940.65(4) $152373.63(3) \quad$ pa

$30693.33(3) \quad 155085.02(3) \quad$ pa

$27940.65(4) \quad 152304.45(5) \quad$ pa

$35440.07(3) \quad 159791.70(4) \quad$ pa *

25969.65(4) 150235.09(3) pa

46354.20(4) $170618.53(5) \quad$ pa

$21190.03(3) \quad 145439.95(4) \quad$ pa

18463.42(5) $142662.22(6) \quad$ pa

$22138.98(2) \quad 146330.41(2) \quad$ pa

$33303.67(6) \quad 157481.05(5) \quad$ pa

$21190.03(3) \quad 145359.05(2) \quad \mathrm{pa}$

30693.33(3) $\quad 154852.06(2) \quad$ pa

$39120.48(2) \quad 163205.61(3) \quad$ pa

$22138.98(2) \quad 146186.18(3) \quad$ pa

33303.67(6) 157327.79(6) pa

$14546.32(4) \quad 138529.81(3) \quad$ pa $\quad$ C II

8526.56(1) 132468.31(2) $\quad$ pa *

$5178.26(2) \quad 129069.98(3) \quad$ pa

27670.52(2) 151395.26(3) pa

$28652.05(5) \quad 152304.45(5) \quad$ pa 


\begin{tabular}{|c|c|c|c|c|c|c|c|c|c|c|c|c|c|}
\hline 3623 & 1820 & 809.290 & 123565.05 & 809.2885 & 0.0014 & -0.28 & 0.002 & 10 & 23 & $5 d^{6}$ & $19096.89(6)$ & $142662.22(6)$ & pa \\
\hline 57 & 82 & 809.995 & 123457.51 & 809.9841 & 0.0016 & -1.71 & 0.011 & 13 & 31 & $5 d^{6}$ & $30693.33(3)$ & $154152.55(3)$ & pa* \\
\hline 268 & 1242 & 810.136 & 123436.00 & 810.1356 & 0.0013 & -0.12 & 0.001 & 9 & 39 & $5 d^{6}$ & $21190.03(3)$ & $144626.15(2)$ & pa \\
\hline 183 & 430 & 810.284 & 123413.53 & 810.2815 & 0.0016 & -0.37 & 0.002 & 13 & 43 & $5 d^{6}$ & $28959.73(3)$ & $152373.63(3)$ & pa \\
\hline 127 & 72 & 810.849 & 123327.48 & 810.8390 & 0.0021 & -1.56 & 0.010 & 9 & 20 & $5 d^{6}$ & $46354.20(4)$ & $169683.24(3)$ & pa* \\
\hline 762 & 697 & 811.327 & 123254.91 & 811.3330 & 0.0030 & 0.95 & -0.006 & 8 & 7 & $5 d^{6}$ & $39120.48(2)$ & $162374.44(3)$ & pa \\
\hline 599 & 1782 & 811.383 & 123246.31 & 811.3862 & 0.0011 & 0.44 & -0.003 & 10 & 39 & $5 d^{6}$ & $5824.11(3)$ & $129069.98(3)$ & pa \\
\hline 143 & 160 & 811.556 & 123220.01 & 811.5561 & 0.0016 & -0.06 & 0.000 & 9 & 25 & $5 d^{6}$ & $22138.98(2)$ & $145359.05(2)$ & pa \\
\hline 254 & 1345 & 811.679 & 123201.46 & 811.6747 & 0.0012 & -0.60 & 0.004 & 13 & 26 & $5 d^{6}$ & $0.00(4)$ & $123202.06(3)$ & pa \\
\hline 561 & 1625 & 811.988 & 123154.50 & 811.9894 & 0.0013 & 0.18 & -0.001 & 12 & 32 & $5 d^{6}$ & $14546.32(4)$ & $137700.64(3)$ & pa \\
\hline 167 & 835 & 812.103 & 123137.12 & 812.0867 & 0.0012 & -2.44 & 0.016 & 11 & 32 & $5 d^{6}$ & $14546.32(4)$ & $137685.88(5)$ & pa* \\
\hline 194 & 835 & 812.103 & 123137.12 & 812.1158 & 0.0015 & 1.97 & -0.013 & 12 & 31 & $5 d^{6}$ & $30693.33(3)$ & $153828.48(4)$ & pa* \\
\hline 307 & 92 & 812.226 & 123118.50 & 812.2246 & 0.0023 & -0.15 & 0.001 & 9 & 7 & $5 d^{6}$ & $46564.59(2)$ & $169683.24(3)$ & pa \\
\hline 196 & 505 & 812.615 & 123059.57 & 812.6151 & 0.0014 & 0.08 & -0.001 & 10 & 36 & $5 d^{6}$ & $27940.65(4)$ & $151000.14(5)$ & pa \\
\hline 546 & 455 & 812.918 & 123013.67 & 812.9124 & 0.0030 & -0.82 & 0.005 & 5 & 7 & $5 d^{6}$ & $39120.48(2)$ & $162134.97(2)$ & pa \\
\hline 381 & 872 & 813.216 & 122968.62 & 813.2192 & 0.0015 & 0.55 & -0.004 & 12 & 30 & $5 d^{6}$ & $25969.65(4)$ & $148937.72(5)$ & pa \\
\hline 86 & 242 & 814.571 & 122763.99 & 814.5618 & 0.0014 & -1.41 & 0.009 & 11 & 36 & $5 d^{6}$ & $27940.65(4)$ & $150706.05(4)$ & pa $* 100$ \\
\hline 417 & 1605 & 814.789 & 122731.16 & 814.7898 & 0.0013 & 0.12 & -0.001 & 9 & 32 & $5 d^{6}$ & $14546.32(4)$ & $137277.36(4)$ & pa \\
\hline 70 & 302 & 814.903 & 122713.95 & 814.9114 & 0.0014 & 1.22 & -0.008 & 9 & 32 & $5 d^{6}$ & $28652.05(5)$ & $151364.78(6)$ & pa \\
\hline 436 & 1190 & 815.145 & 122677.51 & 815.1438 & 0.0012 & -0.22 & 0.001 & 15 & 37 & $5 d^{6}$ & $18463.42(5)$ & $141141.16(4)$ & pa \\
\hline 426 & 1312 & 815.356 & 122645.80 & 815.3544 & 0.0013 & -0.25 & 0.002 & 8 & 30 & $5 d^{6}$ & $5178.26(2)$ & $127824.31(1)$ & pa \\
\hline 379 & 1250 & 815.903 & 122563.66 & 815.8965 & 0.0015 & -0.91 & 0.006 & 11 & 30 & $5 d^{6}$ & $27670.52(2)$ & $150235.09(3)$ & pa \\
\hline 49 & 55 & 816.331 & 122499.30 & 816.3279 & 0.0020 & -0.50 & 0.003 & 9 & 31 & $5 d^{6}$ & $30693.33(3)$ & $153193.13(2)$ & pa \\
\hline 42 & 132 & 816.415 & 122486.76 & 816.4120 & 0.0016 & -0.41 & 0.003 & 9 & 25 & $5 d^{6}$ & $22138.98(2)$ & $144626.15(2)$ & pa \\
\hline 117 & 635 & 816.675 & 122447.73 & 816.6805 & 0.0023 & 0.82 & -0.005 & 6 & 17 & $5 d^{6}$ & $26238.28(1)$ & $148685.19(2)$ & pa \\
\hline 41 & 112 & 816.752 & 122436.13 & 816.7564 & 0.0014 & 0.60 & -0.004 & 11 & 43 & $5 d^{6}$ & $28959.73(3)$ & $151395.26(3)$ & pa* \\
\hline 49 & 132 & 816.997 & 122399.51 & 817.0011 & 0.0022 & 0.66 & -0.004 & 4 & 17 & $5 d^{6}$ & $26238.28(1)$ & $148637.14(1)$ & pa \\
\hline 98 & 225 & 817.061 & 122389.93 & 817.0660 & 0.0016 & 0.80 & -0.005 & 8 & 30 & $5 d^{6}$ & $25969.65(4)$ & $148358.78(3)$ & pa \\
\hline 54 & 82 & 817.152 & 122376.21 & 817.1608 & 0.0019 & 1.28 & -0.009 & 8 & 23 & $5 d^{6}$ & $37387.78(4)$ & $159762.71(5)$ & pa \\
\hline 196 & 1740 & 817.406 & 122338.21 & 817.4032 & 0.0014 & -0.44 & 0.003 & 7 & 26 & $5 d^{6}$ & $0.00(4)$ & $122338.65(5)$ & pa \\
\hline 369 & 912 & 817.735 & 122289.04 & 817.7355 & 0.0015 & 0.10 & -0.001 & 8 & 31 & $5 d^{6}$ & $30693.33(3)$ & $152982.27(4)$ & pa \\
\hline 151 & 157 & 818.059 & 122240.53 & 818.0519 & 0.0030 & -1.11 & 0.007 & 6 & 23 & $5 d^{6}$ & $37387.78(4)$ & $159629.42(3)$ & pa \\
\hline 167 & 1227 & 818.270 & 122209.04 & 818.2742 & 0.0018 & 0.62 & -0.004 & 7 & 25 & $5 d^{6}$ & $22138.98(2)$ & $144347.40(1)$ & pa \\
\hline 151 & 580 & 819.103 & 122084.81 & 819.0972 & 0.0014 & -0.82 & 0.005 & 14 & 25 & $5 d^{6}$ & $19128.73(2)$ & $141214.36(3)$ & pa \\
\hline 212 & 980 & 819.310 & 122053.98 & 819.3095 & 0.0014 & -0.02 & 0.000 & 11 & 32 & $5 d^{6}$ & $28652.05(5)$ & $150706.05(4)$ & pa \\
\hline 511 & 1440 & 819.895 & 121966.84 & 819.8944 & 0.0011 & -0.08 & 0.001 & 16 & 39 & $5 d^{6}$ & $21190.03(3)$ & $143156.95(4)$ & pa \\
\hline 132 & 500 & 819.924 & 121962.46 & 819.9229 & 0.0025 & -0.23 & 0.002 & 11 & 8 & $5 d^{6}$ & $34466.53(1)$ & $156429.22(2)$ & pa \\
\hline 229 & 172 & 820.244 & 121914.91 & 820.2434 & 0.0015 & -0.12 & 0.001 & 11 & 30 & $5 d^{6}$ & $25969.65(4)$ & $147884.68(4)$ & pa \\
\hline 914 & 1922 & 820.334 & 121901.52 & 820.3291 & 0.0013 & -0.77 & 0.005 & 14 & 26 & $5 d^{6}$ & $0.00(4)$ & $121902.29(3)$ & pa \\
\hline 136 & 310 & 820.462 & 121882.48 & 820.4592 & 0.0012 & -0.49 & 0.003 & 12 & 32 & $5 d^{6}$ & $14546.32(4)$ & $136429.29(5)$ & pa \\
\hline 350 & 1172 & 821.061 & 121793.63 & 821.0614 & 0.0014 & 0.06 & 0.000 & 11 & 30 & $5 d^{6}$ & $25969.65(4)$ & $147763.22(4)$ & pa \\
\hline 59 & 77 & 821.508 & 121727.29 & 821.5092 & 0.0018 & 0.11 & -0.001 & 11 & 29 & $5 d^{6}$ & $35440.07(3)$ & $157167.25(4)$ & pa \\
\hline 49 & 142 & 821.836 & 121678.76 & 821.8258 & 0.0017 & -1.53 & 0.010 & 13 & 31 & $5 d^{6}$ & $30693.33(3)$ & $152373.63(3)$ & pa* \\
\hline 228 & 585 & 821.967 & 121659.36 & 821.9661 & 0.0031 & -0.16 & 0.001 & 5 & 29 & $5 d^{6}$ & $35440.07(3)$ & $157099.59(2)$ & pa \\
\hline
\end{tabular}




$\begin{array}{rrrr}87 & 210 & 822.414 & 121593.25 \\ 14 & 20 & 822.712 & 121549.28 \\ 31 & 52 & 822.817 & 121533.71 \\ 272 & 967 & 824.104 & 121343.95 \\ 1092 & 1230 & 824.288 & 121316.76 \\ 97 & 1252 & 824.571 & 121275.24 \\ 458 & 1252 & 824.571 & 121275.24 \\ 1016 & 1177 & 826.139 & 121045.04 \\ 103 & 137 & 826.472 & 120996.28 \\ 171 & 892 & 826.958 & 120925.15 \\ 169 & 432 & 827.812 & 120800.43 \\ 327 & 280 & 828.143 & 120752.11 \\ 167 & 782 & 828.328 & 120725.08 \\ 107 & 207 & 828.586 & 120687.47 \\ 109 & 180 & 828.770 & 120660.78 \\ 156 & 577 & 828.892 & 120642.99 \\ 137 & 615 & 828.975 & 120630.94 \\ 98 & 527 & 830.118 & 120464.83 \\ 13 & 35 & 830.655 & 120386.88 \\ 80 & 1960 & 831.197 & 120308.44 \\ 99 & 285 & 831.828 & 120217.22 \\ 45 & 40 & 833.161 & 120024.89 \\ 126 & 87 & 833.243 & 120013.08 \\ 154 & 77 & 833.830 & 119928.45 \\ 577 & 1137 & 834.108 & 119888.48 \\ 115 & 205 & 834.576 & 119821.34 \\ 171 & 225 & 834.871 & 119778.93 \\ 284 & 1472 & 835.294 & 119718.37 \\ 1039 & 1870 & 835.721 & 119657.14 \\ 54 & 122 & 836.433 & 119555.35 \\ 69 & 195 & 836.531 & 119541.28 \\ 182 & 1465 & 836.969 & 119478.79 \\ 338 & 827 & 837.440 & 119411.54 \\ 243 & 1522 & 837.517 & 119400.53 \\ 493 & 1777 & 837.756 & 119366.55 \\ 61 & 397 & 837.963 & 119336.95 \\ 31 & 395 & 838.234 & 119298.40 \\ 44 & 42 & 838.464 & 119265.69 \\ 145 & 422 & 838.703 & 119231.69 \\ 231 & 1525 & 838.770 & 119222.21 \\ 77 & 12 & 839.181 & 119163.85 \\ 322 & 1612 & 839.493 & 119119.53 \\ 151 & 970 & 839.804 & 119075.46 \\ 805 & 2180 & 841.280 & 118866.48\end{array}$

$\begin{array}{llll}822.4089 & 0.0018 & -0.76\end{array}$ $\begin{array}{llll}822.7058 & 0.0016 & -0.85\end{array}$ $\begin{array}{llll}822.8085 & 0.0012 & -1.25\end{array}$ $\begin{array}{lll}824.1000 & 0.0015 & -0.55\end{array}$ $\begin{array}{llll}824.2835 & 0.0015 & -0.73\end{array}$ $\begin{array}{llll}824.5628 & 0.0011 & -1.14\end{array}$ $\begin{array}{lll}824.5698 & 0.0015 & -0.12\end{array}$ $\begin{array}{lll}826.1386 & 0.0022 & -0.02\end{array}$ $\begin{array}{llll}826.4663 & 0.0015 & -0.79\end{array}$ $\begin{array}{llll}826.9533 & 0.0013 & -0.66\end{array}$ $\begin{array}{llll}827.8108 & 0.0017 & -0.12\end{array}$ $\begin{array}{llll}828.1359 & 0.0018 & -1.02\end{array}$ $\begin{array}{llll}828.3212 & 0.0017 & -1.04\end{array}$ $\begin{array}{lll}828.5810 & 0.0016 & -0.79\end{array}$ $\begin{array}{lll}828.7673 & 0.0013 & -0.35\end{array}$ $\begin{array}{llll}828.8924 & 0.0013 & 0.07\end{array}$ $\begin{array}{llll}828.9721 & 0.0014 & -0.37\end{array}$ $\begin{array}{llll}830.1154 & 0.0016 & -0.35\end{array}$ $\begin{array}{lll}830.6646 & 0.0040 & 1.35\end{array}$ $\begin{array}{lll}831.2220 & 0.0019 & 3.64\end{array}$ $\begin{array}{lll}831.8324 & 0.0016 & 0.69\end{array}$ $\begin{array}{lll}833.1644 & 0.0013 & 0.56\end{array}$ $\begin{array}{lll}833.2450 & 0.0015 & 0.37\end{array}$ $\begin{array}{llll}833.8300 & 0.0020 & -0.07\end{array}$ $\begin{array}{llll}834.1068 & 0.0021 & -0.24\end{array}$ $\begin{array}{llll}834.5673 & 0.0014 & -1.23\end{array}$ $\begin{array}{llll}834.8676 & 0.0019 & -0.54\end{array}$ $\begin{array}{llll}835.2442 & 0.0023 & -7.09\end{array}$ $\begin{array}{llll}835.7166 & 0.0012 & -0.64\end{array}$ $\begin{array}{llll}836.4309 & 0.0013 & -0.26\end{array}$ $\begin{array}{llll}836.5278 & 0.0017 & -0.47\end{array}$ $\begin{array}{lll}836.9672 & 0.0012 & -0.19\end{array}$ $\begin{array}{llll}837.4368 & 0.0031 & -0.45\end{array}$ $\begin{array}{lll}837.5160 & 0.0011 & -0.17\end{array}$ $\begin{array}{lll}837.7527 & 0.0015 & -0.41\end{array}$ $\begin{array}{lll}837.9639 & 0.0012 & 0.06\end{array}$ $\begin{array}{llll}838.2388 & 0.0017 & 0.65\end{array}$ $\begin{array}{llll}838.4667 & 0.0020 & 0.37\end{array}$ $\begin{array}{lll}838.6966 & 0.0015 & -0.94\end{array}$ $\begin{array}{lll}838.7682 & 0.0012 & -0.25\end{array}$ $\begin{array}{lll}839.1765 & 0.0040 & -0.60\end{array}$ $\begin{array}{lll}839.4842 & 0.0017 & -1.24\end{array}$ $\begin{array}{lll}839.8042 & 0.0016 & 0.08\end{array}$ $\begin{array}{lll}841.2802 & 0.0012 & 0.02\end{array}$ $\begin{array}{llll}0.005 & 8 & 30 & 5 \mathrm{~d}^{6}\end{array}$ $\begin{array}{llll}0.006 & 8 & 25 & 5 \mathrm{~d}^{6}\end{array}$

$\begin{array}{llll}0.008 & 15 & 37 & 5 d^{6}\end{array}$

$\begin{array}{llll}0.004 & 10 & 25 & 5 \mathrm{~d}^{6}\end{array}$

$\begin{array}{llll}0.005 & 11 & 29 & 5 \mathrm{~d}^{6}\end{array}$

$\begin{array}{llll}0.008 & 16 & 37 & 5 d^{6}\end{array}$

$\begin{array}{lllll}0.001 & 11 & 43 & 5 \mathrm{~d}^{6}\end{array}$

$\begin{array}{llll}0.000 & 4 & 10 & 5 \mathrm{~d}^{6}\end{array}$

$\begin{array}{llll}0.005 & 12 & 36 & 5 d^{6}\end{array}$

$\begin{array}{llll}0.005 & 11 & 25 & 5 \mathrm{~d}^{6}\end{array}$

$\begin{array}{llll}0.001 & 12 & 23 & 5 d^{6}\end{array}$

$\begin{array}{llll}0.007 & 7 & 20 & 5 \mathrm{~d}^{6}\end{array}$

$\begin{array}{llll}0.007 & 13 & 25 & 5 d^{6}\end{array}$

$\begin{array}{lllll}0.005 & 8 & 30 & 5 \mathrm{~d}^{6}\end{array}$

$\begin{array}{llll}0.002 & 9 & 32 & 5 d^{6}\end{array}$

$\begin{array}{llll}-0.001 & 16 & 23 & 5 d^{6}\end{array}$

$\begin{array}{llll}0.003 & 5 & 37 & 5 \mathrm{~d}^{6}\end{array}$

$\begin{array}{llll}0.002 & 8 & 30 & 5 \mathrm{~d}^{6}\end{array}$

$\begin{array}{llll}-0.009 & 12 & 8 & 5 d^{6}\end{array}$

$\begin{array}{llll}-0.025 & 8 & 43 & 5 d^{6}\end{array}$

$\begin{array}{llll}-0.005 & 17 & 30 & 5 d^{6}\end{array}$

$\begin{array}{llll}-0.004 & 14 & 39 & 5 d^{6}\end{array}$

$\begin{array}{llll}-0.003 & 11 & 31 & 5 \mathrm{~d}^{6}\end{array}$

$\begin{array}{llll}0.000 & 5 & 20 & 5 \mathrm{~d}^{6}\end{array}$

$\begin{array}{llll}0.002 & 6 & 12 & 5 d^{6}\end{array}$

$\begin{array}{lllll}0.009 & 11 & 36 & 5 d^{6}\end{array}$

$\begin{array}{llll}0.004 & 11 & 23 & 5 \mathrm{~d}^{6} \\ 0.049 & 6 & 43 & 5 \mathrm{~d}^{6}\end{array}$

$\begin{array}{llll}0.049 & 6 & 43 & 5 \mathrm{~d}^{6}\end{array}$

$\begin{array}{llll}0.004 & 10 & 37 & 5 d^{6}\end{array}$

$\begin{array}{llll}0.002 & 17 & 30 & 5 \mathrm{~d}^{6}\end{array}$

$\begin{array}{llll}0.003 & 11 & 31 & 5 \mathrm{~d}^{6}\end{array}$

$\begin{array}{llll}0.001 & 16 & 32 & 5 \mathrm{~d}^{6}\end{array}$

$\begin{array}{llll}0.003 & 12 & 29 & 5 \mathrm{~d}^{6}\end{array}$

$\begin{array}{llll}0.001 & 15 & 39 & 5 d^{6}\end{array}$

$\begin{array}{llll}0.003 & 5 & 23 & 5 \mathrm{~d}^{6}\end{array}$

$\begin{array}{llll}0.000 & 15 & 39 & 5 d^{6}\end{array}$

$\begin{array}{llll}-0.005 & 8 & 14 & 5 d^{6}\end{array}$

$\begin{array}{llll}-0.003 & 9 & 14 & 5 d^{5} 6 \mathrm{~s}\end{array}$

$\begin{array}{llll}0.007 & 11 & 32 & 5 d^{6}\end{array}$

$\begin{array}{llll}0.002 & 11 & 37 & 5 \mathrm{~d}^{6}\end{array}$

$\begin{array}{llll}0.004 & 5 & 3 & 5 \mathrm{~d}^{6}\end{array}$

$\begin{array}{llll}0.009 & 9 & 17 & 5 \mathrm{~d}^{6}\end{array}$

$\begin{array}{llll}-0.001 & 14 & 25 & 5 \mathrm{~d}^{6}\end{array}$

$\begin{array}{llll}0.000 & 8 & 32 & 5 \mathrm{~d}^{6}\end{array}$

20
27670.52(2) $\quad 149264.53(2) \quad$ pa

19128.73(2) 140678.86(1) pa *

18463.42(5) $139998.38(4) \quad$ pa

19128.73(2) $140473.23(2) \quad$ pa

$35440.07(3) \quad 156757.56(3) \quad$ pa

$18463.42(5) \quad 139739.81(5) \quad$ pa * 200

28959.73(3) 150235.09(3) pa

$\begin{array}{lll}53982.00(4) & 175027.06(4) & \text { pa }\end{array}$

$27940.65(4) \quad 148937.72(5) \quad$ pa

19128.73(2) $140054.55(3) \quad$ pa

$37387.78(4) \quad 158188.33(4) \quad$ pa

46354.20(4) 167107.33(4) pa

22138.98(2) $142865.10(2) \quad$ pa

$27670.52(2) \quad 148358.78(3) \quad$ pa

28652.05(5) $149313.18(6) \quad \mathrm{pa}$

$19096.89(6) \quad 139739.81(5) \quad$ pa

18463.42(5) $139094.74(6) \quad$ pa

$27670.52(2) \quad 148135.70(2) \quad$ pa

$34466.53(1) \quad 154852.06(2) \quad$ pa *

$28959.73(3) \quad 149264.53(2) \quad$ pa $* 400, m$

$25969.65(4) \quad 146186.18(3) \quad$ pa

$21190.03(3) \quad 141214.36(3) \quad$ pa

$30693.33(3) \quad 150706.05(4) \quad$ pa

46354.20(4) $166282.72(5) \quad$ pa

$33303.67(6) \quad 153192.39(7) \quad$ pa

$27940.65(4) \quad 147763.22(4) \quad$ pa

$37387.78(4) \quad 157167.25(4) \quad$ pa

28959.73(3) $148685.19(2) \quad$ pa * 600, m(O III)

$18463.42(5) \quad 138121.21(6) \quad$ pa

$25969.65(4) \quad 145525.25(5) \quad$ pa

30693.33(3) $\quad 150235.09(3) \quad$ pa

14546.32(4) 134025.31(4) $\quad$ pa

$35440.07(3) \quad 154852.06(2) \quad$ pa

5824.11(3) 125224.81(4) $\quad$ pa

19096.89(6) 138463.86(7) pa

$21190.03(3) \quad 140526.92(3) \quad$ pa

8526.56(1) 127824.31(1) pa

50417.92(2) 169683.24(3) pa *

$28652.05(5) \quad 147884.68(4)$ pa 170

$18463.42(5) \quad 137685.88(5) \quad$ pa

42519.60(1) 161684.04(1) pa *

$26238.28(1) \quad 145359.05(2)$ pa 900

22138.98(2) 141214.36(3) pa

$14546.32(4) \quad 133412.78(5) \quad$ pa 


$\begin{array}{rrrr}110 & 770 & 841.696 & 118807.71 \\ 94 & 132 & 842.372 & 118712.44 \\ 63 & 272 & 842.747 & 118659.64 \\ 39 & 327 & 843.373 & 118571.53 \\ 88 & 42 & 843.576 & 118542.91 \\ 77 & 117 & 843.777 & 118514.68 \\ 257 & 1477 & 843.945 & 118491.18 \\ 102 & 1485 & 844.679 & 118388.17 \\ 290 & 1485 & 844.679 & 118388.17 \\ 625 & 1485 & 844.679 & 118388.17 \\ 295 & 220 & 844.992 & 118344.30 \\ 434 & 2197 & 845.093 & 118330.13 \\ 112 & 812 & 845.705 & 118244.61 \\ 140 & 942 & 846.549 & 118126.67 \\ 134 & 475 & 846.680 & 118108.33 \\ 19 & 95 & 846.737 & 118100.49 \\ 102 & 162 & 847.611 & 117978.69 \\ 304 & 1017 & 847.659 & 117972.04 \\ 126 & 1060 & 847.699 & 117966.46 \\ 143 & 390 & 848.427 & 117865.25 \\ 39 & 125 & 849.241 & 117752.21 \\ 54 & 110 & 849.641 & 117696.80 \\ 228 & 1087 & 850.457 & 117583.83 \\ 64 & 572 & 850.578 & 117567.17 \\ 184 & 690 & 850.760 & 117541.98 \\ 77 & 205 & 851.481 & 117442.47 \\ 75 & 792 & 851.634 & 117421.26 \\ 37 & 82 & 852.003 & 117370.42 \\ 48 & 195 & 852.281 & 117332.25 \\ 25 & 125 & 853.052 & 117226.13 \\ 107 & 767 & 853.361 & 117183.76 \\ 69 & 642 & 853.736 & 117132.26 \\ 24 & 80 & 855.190 & 116933.02 \\ 98 & 865 & 855.272 & 116921.88 \\ 142 & 152 & 855.537 & 116885.64 \\ 200 & 242 & 855.786 & 116851.64 \\ 136 & 1895 & 856.742 & 116721.21 \\ 229 & 200 & 857.328 & 116641.45 \\ 502 & 817 & 857.361 & 116637.02 \\ 69 & 400 & 857.812 & 116575.63 \\ 62 & 370 & 858.293 & 116510.36 \\ 157 & 1257 & 858.543 & 116476.44 \\ 123 & 802 & 859.171 & 116391.20 \\ 276 & 950 & 859.421 & 116357.35\end{array}$

$\begin{array}{llll}841.6917 & 0.0012 & -0.64\end{array}$ $\begin{array}{llll}842.3714 & 0.0017 & -0.04\end{array}$ $\begin{array}{llll}842.7448 & 0.0017 & -0.25\end{array}$ $\begin{array}{llll}843.3752 & 0.0020 & 0.33\end{array}$ $\begin{array}{llll}843.5731 & 0.0040 & -0.46\end{array}$ $\begin{array}{lll}843.7704 & 0.0016 & -0.98\end{array}$ $\begin{array}{llll}843.9423 & 0.0013 & -0.33\end{array}$ $\begin{array}{llll}844.6806 & 0.0016 & 0.23\end{array}$ $\begin{array}{llll}844.6773 & 0.0017 & -0.24\end{array}$ $\begin{array}{llll}844.6693 & 0.0021 & -1.36\end{array}$ $\begin{array}{llll}844.9849 & 0.0030 & -1.01\end{array}$ $\begin{array}{llll}845.0910 & 0.0013 & -0.32\end{array}$ $\begin{array}{lll}845.6979 & 0.0017 & -0.92\end{array}$ $\begin{array}{lll}846.5404 & 0.0016 & -1.18\end{array}$ $\begin{array}{llll}846.6747 & 0.0021 & -0.79\end{array}$ $\begin{array}{llll}846.7377 & 0.0014 & 0.16\end{array}$ $\begin{array}{llll}847.6077 & 0.0030 & -0.42\end{array}$ $\begin{array}{llll}847.6583 & 0.0015 & -0.02\end{array}$ $\begin{array}{lll}847.7028 & 0.0013 & 0.59\end{array}$ $\begin{array}{lll}848.4199 & 0.0015 & -0.92\end{array}$ $\begin{array}{lll}849.2348 & 0.0022 & -0.85\end{array}$ $\begin{array}{lll}849.6376 & 0.0021 & -0.44\end{array}$ $\begin{array}{llll}850.4515 & 0.0013 & -0.77\end{array}$ $\begin{array}{lll}850.5714 & 0.0013 & -0.86\end{array}$ $\begin{array}{lll}850.7583 & 0.0016 & -0.22\end{array}$ $\begin{array}{lll}851.4815 & 0.0018 & 0.11\end{array}$ $\begin{array}{lll}851.6253 & 0.0013 & -1.26\end{array}$ $\begin{array}{lll}852.0015 & 0.0017 & -0.26\end{array}$ $\begin{array}{llll}852.2795 & 0.0015 & -0.15\end{array}$ $\begin{array}{lll}853.0498 & 0.0016 & -0.32\end{array}$ $\begin{array}{lll}853.3542 & 0.0013 & -0.87\end{array}$ $\begin{array}{lll}853.7375 & 0.0012 & 0.24\end{array}$ $\begin{array}{llll}855.1865 & 0.0019 & -0.54\end{array}$ $\begin{array}{llll}855.2697 & 0.0012 & -0.30\end{array}$ $\begin{array}{lll}855.5304 & 0.0019 & -0.91\end{array}$ $\begin{array}{rrr}855.7877 & 0.0021 & 0.23\end{array}$ $\begin{array}{lll}856.7216 & 0.0013 & -2.82\end{array}$ $\begin{array}{lll}857.3313 & 0.0024 & 0.42\end{array}$ $\begin{array}{lll}857.3643 & 0.0022 & 0.49\end{array}$ $\begin{array}{lll}857.8024 & 0.0016 & -1.33\end{array}$ $\begin{array}{lll}858.2909 & 0.0014 & -0.25\end{array}$ $\begin{array}{llll}858.5149 & 0.0013 & -3.78\end{array}$ $\begin{array}{lll}859.1742 & 0.0017 & 0.37\end{array}$ $\begin{array}{llll}859.4149 & 0.0024 & -0.88\end{array}$ $\begin{array}{llll}0.005 & 15 & 39 & 5 d^{6}\end{array}$ $\begin{array}{llll}0.000 & 13 & 29 & 5 \mathrm{~d}^{6}\end{array}$

$\begin{array}{llll}0.002 & 6 & 30 & 5 d^{6}\end{array}$

$\begin{array}{llll}-0.002 & 8 & 31 & 5 \mathrm{~d}^{6}\end{array}$

$\begin{array}{llll}0.003 & 9 & 3 & 5 \mathrm{~d}^{6}\end{array}$

$\begin{array}{llll}0.007 & 17 & 30 & 5 d^{6}\end{array}$

$\begin{array}{llll}0.002 & 14 & 32 & 5 \mathrm{~d}^{6}\end{array}$

$\begin{array}{llll}-0.002 & 15 & 25 & 5 \mathrm{~d}^{6} \\ 0.002 & 12 & 29 & 5 \mathrm{~d}^{6}\end{array}$

$\begin{array}{llll}0.002 & 12 & 29 & 5 \mathrm{~d}^{6}\end{array}$

$\begin{array}{llll}0.010 & 8 & 20 & 5 \mathrm{~d}^{6}\end{array}$

$\begin{array}{llll}0.007 & 7 & 7 & 5 \mathrm{~d}^{6}\end{array}$

$\begin{array}{llll}0.002 & 13 & 26 & 5 \mathrm{~d}^{6}\end{array}$

$\begin{array}{lllll}0.007 & 17 & 36 & 5 d^{6}\end{array}$

$\begin{array}{llll}0.008 & 5 & 23 & 5 \mathrm{~d}^{6}\end{array}$

$\begin{array}{llll}0.006 & 7 & 17 & 5 d^{6}\end{array}$

$\begin{array}{llll}-0.001 & 12 & 25 & 5 d^{6}\end{array}$

$\begin{array}{llll}0.003 & 5 & 7 & 5 \mathrm{~d}^{6}\end{array}$

$\begin{array}{llll}0.000 & 14 & 29 & 5 \mathrm{~d}^{6}\end{array}$

$\begin{array}{llll}-0.004 & 12 & 37 & 5 d^{6}\end{array}$

$\begin{array}{llll}0.007 & 7 & 30 & 5 d^{6}\end{array}$

$\begin{array}{llll}0.006 & 9 & 29 & 5 \mathrm{~d}^{6}\end{array}$

$\begin{array}{llll}0.003 & 13 & 23 & 5 d^{6}\end{array}$

$\begin{array}{lllll}0.006 & 17 & 36 & 5 \mathrm{~d}^{6}\end{array}$

$\begin{array}{lllll}0.006 & 9 & 30 & 5 \mathrm{~d}^{6}\end{array}$

$\begin{array}{llll}0.002 & 8 & 29 & 5 d^{6}\end{array}$

$\begin{array}{llll}-0.001 & 8 & 31 & 5 d^{6}\end{array}$

$\begin{array}{llll}0.009 & 8 & 39 & 5 \mathrm{~d}^{6}\end{array}$

$\begin{array}{llll}0.002 & 6 & 43 & 5 \mathrm{~d}^{6}\end{array}$

$\begin{array}{llll}0.001 & 12 & 23 & 5 d^{6}\end{array}$

$\begin{array}{llll}0.002 & 17 & 43 & 5 \mathrm{~d}^{6}\end{array}$

$\begin{array}{llll}0.006 & 15 & 30 & 5 \mathrm{~d}^{6}\end{array}$

$\begin{array}{llll}-0.002 & 17 & 37 & 5 \mathrm{~d}^{6}\end{array}$

$\begin{array}{llll}0.004 & 13 & 29 & 5 \mathrm{~d}^{6}\end{array}$

$\begin{array}{llll}0.002 & 9 & 39 & 5 d^{6}\end{array}$

$\begin{array}{llll}0.007 & 7 & 20 & 5 \mathrm{~d}^{6}\end{array}$

$\begin{array}{llll}-0.002 & 8 & 20 & 5 d^{6}\end{array}$

$\begin{array}{llll}0.021 & 14 & 30 & 5 \mathrm{~d}^{6}\end{array}$

$\begin{array}{llll}-0.003 & 8 & 7 & 5 d^{6}\end{array}$

$\begin{array}{llll}-0.004 & 6 & 10 & 5 d^{6}\end{array}$

$\begin{array}{llll}0.010 & 7 & 43 & 5 \mathrm{~d}^{6}\end{array}$

$\begin{array}{llll}0.002 & 12 & 39 & 5 \mathrm{~d}^{6}\end{array}$

$\begin{array}{llll}0.028 & 16 & 43 & 5 \mathrm{~d}^{6}\end{array}$

$\begin{array}{llll}-0.003 & 10 & 25 & 5 \mathrm{~d}^{6}\end{array}$

$\begin{array}{llll}0.006 & 5 & 12 & 5 \mathrm{~d}^{6}\end{array}$
21190.03(3) $139998.38(4) \quad$ pa

$35440.07(3) \quad 154152.55(3) \quad$ pa

$27670.52(2) \quad 146330.41(2) \quad$ pa

$30693.33(3) \quad 149264.53(2)$ pa 200

42519.60(1) $161062.97(2) \quad$ pa

27670.52(2) 146186.18(3) pa

14546.32(4) $133037.83(3)$ pa

22138.98(2) $140526.92(3) \quad$ pa

$35440.07(3) \quad 153828.48(4) \quad$ pa

46354.20(4) $164743.73(4) \quad$ pa $* 900$

$46564.59(2) \quad 164909.90(2) \quad$ pa

$0.00(4) \quad 118330.45(3) \quad$ pa

27940.65(4) 146186.18(3) pa

19096.89(6) 137224.74(7) pa

26238.28(1) $144347.40(1) \quad$ pa

$19128.73(2) \quad 137229.06(2) \quad$ pa

39120.48(2) $\quad 157099.59(2) \quad$ pa

$35440.07(3) \quad 153412.13(4)$ pa 800

$18463.42(5) \quad 136429.29(5) \quad$ pa $* 500$

$27670.52(2) \quad 145536.69(3) \quad$ pa

$35440.07(3) \quad 153193.13(2) \quad$ pa

37387.78(4) 155085.02(3) pa*

$27940.65(4) \quad 145525.25(5) \quad$ pa

5178.26(2) $\quad 122746.29(2) \quad$ pa

$35440.07(3) \quad 152982.27(4) \quad$ pa

$30693.33(3) \quad 148135.70(2) \quad$ pa

$21190.03(3) \quad 138612.56(2) \quad$ pa

$28959.73(3) \quad 146330.41(2) \quad$ pa

19096.89(6) $136429.29(5) \quad$ pa

$28959.73(3) \quad 146186.18(3) \quad$ pa

$27670.52(2) \quad 144855.15(3) \quad$ pa

$18463.42(5) \quad 135595.44(4) \quad$ pa

$35440.07(3) \quad 152373.63(3) \quad$ pa *

5824.11(3) $\quad 122746.29(2) \quad$ pa

46354.20(4) $163240.75(5) \quad$ pa

46354.20(4) 163205.61(3) pa

$5178.26(2) \quad 121902.29(3) \quad$ pa $* 1200, \mathrm{bl}(\mathrm{Al}$ III $)$

$46564.59(2) \quad 163205.61(3) \quad$ pa

53982.00(4) $170618.53(5) \quad$ pa

$28959.73(3) \quad 145536.69(3) \quad$ pa

21190.03(3) $137700.64(3) \quad$ pa

28959.73(3) 145439.95(4) pa * 600, m(C II)

22138.98(2) 138529.81(3) pa

$33303.67(6) \quad 149661.90(7) \quad$ pa 


\begin{tabular}{|c|c|c|c|c|c|c|c|c|c|c|c|c|c|}
\hline 97 & 397 & 859.468 & 116351.02 & 859.4629 & 0.0013 & -0.71 & 0.005 & 19 & 32 & $5 d^{6}$ & $28652.05(5)$ & $145003.78(4)$ & pa \\
\hline 31 & 87 & 859.903 & 116292.12 & 859.9096 & 0.0015 & 0.82 & -0.006 & 15 & 25 & $5 d^{6}$ & $19128.73(2)$ & $135420.03(3)$ & pa \\
\hline 97 & 1360 & 860.728 & 116180.76 & 860.7222 & 0.0014 & -0.75 & 0.006 & 8 & 30 & $5 d^{6}$ & $5178.26(2)$ & $121359.77(1)$ & pa \\
\hline 29 & 657 & 861.466 & 116081.19 & 861.4884 & 0.0013 & 3.01 & -0.022 & 14 & 39 & $5 d^{6}$ & 5824.11(3) & $121902.29(3)$ & pa $* 300$ \\
\hline 74 & 100 & 861.757 & 116041.98 & 861.7417 & 0.0013 & -2.07 & 0.015 & 19 & 43 & $5 d^{6}$ & $28959.73(3)$ & $145003.78(4)$ & pa $*$ \\
\hline 146 & 417 & 861.905 & 116022.11 & 861.9186 & 0.0023 & 1.87 & -0.014 & 8 & 20 & $5 d^{6}$ & $46354.20(4)$ & $162374.44(3)$ & $\mathrm{pa} * 200$ \\
\hline 40 & 50 & 862.392 & 115956.55 & 862.4021 & 0.0017 & 1.36 & -0.010 & 11 & 29 & $5 d^{6}$ & $35440.07(3)$ & $151395.26(3)$ & pa $*$ \\
\hline 368 & 1235 & 862.845 & 115895.68 & 862.8469 & 0.0014 & 0.26 & -0.002 & 15 & 43 & $5 d^{6}$ & $28959.73(3)$ & $144855.15(3)$ & pa \\
\hline 599 & 1642 & 863.626 & 115790.82 & 863.6235 & 0.0013 & -0.38 & 0.003 & 14 & 36 & $5 d^{6}$ & $27940.65(4)$ & $143731.85(5)$ & pa \\
\hline 22 & 315 & 864.208 & 115712.82 & 864.2038 & 0.0013 & -0.62 & 0.005 & 11 & 30 & $5 d^{6}$ & $5178.26(2)$ & $120891.70(2)$ & pa \\
\hline 168 & 130 & 864.298 & 115700.82 & 864.2950 & 0.0020 & -0.42 & 0.003 & 9 & 10 & $5 d^{6}$ & $53982.00(4)$ & $169683.24(3)$ & pa \\
\hline 35 & 232 & 864.556 & 115666.32 & 864.5552 & 0.0015 & -0.10 & 0.001 & 9 & 43 & $5 d^{6}$ & $28959.73(3)$ & $144626.15(2)$ & pa \\
\hline 91 & 255 & 865.095 & 115594.28 & 865.0931 & 0.0018 & -0.21 & 0.002 & 8 & 23 & $5 d^{6}$ & $37387.78(4)$ & $152982.27(4)$ & pa \\
\hline 62 & 285 & 865.215 & 115578.18 & 865.2098 & 0.0016 & -0.73 & 0.005 & 13 & 25 & $5 d^{6}$ & $19128.73(2)$ & $134707.64(2)$ & pa \\
\hline 92 & 702 & 865.339 & 115561.61 & 865.3372 & 0.0012 & -0.28 & 0.002 & 16 & 37 & $5 d^{6}$ & $18463.42(5)$ & $134025.31(4)$ & pa \\
\hline 55 & 32 & 865.448 & 115547.03 & 865.4426 & 0.0020 & -0.78 & 0.006 & 9 & 20 & $5 d^{6}$ & $46354.20(4)$ & $161902.01(4)$ & $\mathrm{pa} *$ \\
\hline 80 & 32 & 865.734 & 115508.93 & 865.7372 & 0.0022 & 0.44 & -0.003 & 8 & 10 & $5 d^{6}$ & $53982.00(4)$ & $169490.49(5)$ & pa \\
\hline 119 & 1132 & 866.188 & 115448.45 & 866.1885 & 0.0012 & 0.14 & -0.001 & 8 & 37 & $5 d^{6}$ & $18463.42(5)$ & $133911.73(6)$ & pa \\
\hline 132 & 647 & 867.560 & 115265.87 & 867.5587 & 0.0017 & -0.11 & 0.001 & 11 & 29 & $5 d^{6}$ & $35440.07(3)$ & $150706.05(4)$ & pa \\
\hline 157 & 750 & 867.846 & 115227.86 & 867.8409 & 0.0024 & -0.63 & 0.005 & 5 & 17 & $5 d^{6}$ & $26238.28(1)$ & $141466.77(2)$ & pa \\
\hline 25 & 195 & 867.944 & 115214.79 & 867.9327 & 0.0013 & -1.51 & 0.011 & 16 & 36 & $5 d^{6}$ & $27940.65(4)$ & $143156.95(4)$ & pa* \\
\hline 94 & 1150 & 868.096 & 115194.59 & 868.0964 & 0.0016 & 0.01 & 0.000 & 13 & 30 & $5 d^{6}$ & $27670.52(2)$ & $142865.10(2)$ & pa \\
\hline 137 & 262 & 868.665 & 115119.24 & 868.6629 & 0.0030 & -0.21 & 0.002 & 5 & 7 & $5 d^{6}$ & $46564.59(2)$ & $161684.04(1)$ & pa \\
\hline 137 & 1377 & 869.056 & 115067.44 & 869.0544 & 0.0013 & -0.15 & 0.001 & 11 & 39 & $5 d^{6}$ & $5824.11(3)$ & $120891.70(2)$ & pa \\
\hline 27 & 47 & 869.460 & 115013.96 & 869.4557 & 0.0050 & -0.53 & 0.004 & 6 & 20 & $5 d^{6}$ & $46354.20(4)$ & $161368.69(3)$ & pa $*$ \\
\hline 20 & 32 & 870.200 & 114916.16 & 870.1958 & 0.0017 & -0.51 & 0.004 & 15 & 23 & $5 d^{6}$ & $37387.78(4)$ & $152304.45(5)$ & $\mathrm{pa} *$ \\
\hline 122 & 120 & 870.729 & 114846.34 & 870.7202 & 0.0017 & -1.11 & 0.008 & 7 & 16 & $5 d^{6}$ & $52259.88(3)$ & $167107.33(4)$ & pa \\
\hline 25 & 117 & 870.972 & 114814.27 & 870.9676 & 0.0015 & -0.57 & 0.004 & 8 & 23 & $5 d^{6}$ & $19096.89(6)$ & $133911.73(6)$ & pa \\
\hline 75 & 395 & 871.102 & 114797.16 & 871.0953 & 0.0031 & -0.84 & 0.006 & 8 & 8 & $5 d^{6}$ & $34466.53(1)$ & $149264.53(2)$ & pa \\
\hline 23 & 57 & 872.103 & 114665.41 & 872.1003 & 0.0017 & -0.31 & 0.002 & 9 & 31 & $5 d^{6}$ & $30693.33(3)$ & $145359.05(2)$ & pa * \\
\hline 80 & 805 & 872.932 & 114556.41 & 872.9258 & 0.0015 & -0.86 & 0.007 & 15 & 30 & $5 d^{6}$ & $25969.65(4)$ & $140526.92(3)$ & pa \\
\hline 71 & 362 & 873.329 & 114504.37 & 873.3251 & 0.0013 & -0.53 & 0.004 & 16 & 32 & $5 d^{6}$ & $28652.05(5)$ & $143156.95(4)$ & pa \\
\hline 83 & 137 & 873.371 & 114498.87 & 873.3748 & 0.0030 & 0.48 & -0.004 & 9 & 7 & $5 d^{6}$ & $46564.59(2)$ & $161062.97(2)$ & pa \\
\hline 46 & 197 & 873.816 & 114440.58 & 873.8159 & 0.0019 & 0.00 & 0.000 & 8 & 17 & $5 d^{6}$ & $26238.28(1)$ & $140678.86(1)$ & pa \\
\hline 45 & 220 & 874.084 & 114405.48 & 874.0845 & 0.0013 & 0.07 & -0.001 & 17 & 39 & $5 d^{6}$ & $21190.03(3)$ & $135595.44(4)$ & pa \\
\hline 57 & 342 & 874.812 & 114310.30 & 874.8107 & 0.0014 & -0.14 & 0.001 & 19 & 31 & $5 d^{6}$ & $30693.33(3)$ & $145003.78(4)$ & pa 150 \\
\hline 30 & 130 & 875.414 & 114231.68 & 875.4268 & 0.0014 & 1.68 & -0.013 & 15 & 39 & $5 d^{6}$ & $21190.03(3)$ & $135420.03(3)$ & pa $*$ \\
\hline 25 & 52 & 875.675 & 114197.59 & 875.6781 & 0.0013 & 0.37 & -0.003 & 16 & 43 & $5 d^{6}$ & $28959.73(3)$ & $143156.95(4)$ & pa * \\
\hline 13 & 12 & 875.942 & 114162.81 & 875.9496 & 0.0016 & 0.99 & -0.008 & 15 & 31 & $5 d^{6}$ & $30693.33(3)$ & $144855.15(3)$ & pa * \\
\hline 82 & 1145 & 876.614 & 114075.31 & 876.6134 & 0.0016 & -0.06 & 0.000 & 11 & 25 & $5 d^{6}$ & $19128.73(2)$ & $133204.10(2)$ & pa \\
\hline 44 & 182 & 876.981 & 114027.50 & 876.9720 & 0.0014 & -1.23 & 0.009 & 15 & 30 & $5 d^{6}$ & $25969.65(4)$ & $139998.38(4)$ & pa \\
\hline 66 & 410 & 877.118 & 114009.69 & 877.1147 & 0.0014 & -0.48 & 0.004 & 10 & 32 & $5 d^{6}$ & $28652.05(5)$ & $142662.22(6)$ & pa \\
\hline 55 & 630 & 877.602 & 113946.84 & 877.6018 & 0.0013 & -0.05 & 0.000 & 14 & 32 & $5 d^{6}$ & $14546.32(4)$ & $128493.21(4)$ & pa \\
\hline 261 & 957 & 877.886 & 113910.02 & 877.8890 & 0.0040 & 0.40 & -0.003 & 11 & 3 & $5 d^{6}$ & $42519.60(1)$ & $156429.22(2)$ & pa \\
\hline
\end{tabular}




\begin{tabular}{|c|c|c|c|c|c|c|c|c|c|c|c|c|c|}
\hline 236 & 470 & 878.326 & 113852.93 & 878.3201 & 0.0019 & -0.78 & 0.006 & 7 & 20 & $5 d^{6}$ & $46354.20(4)$ & $160207.91(4)$ & pa \\
\hline 33 & 45 & 878.540 & 113825.17 & 878.5458 & 0.0022 & 0.71 & -0.005 & 8 & 29 & $5 d^{6}$ & $35440.07(3)$ & $149264.53(2)$ & pa \\
\hline 8 & 87 & 878.959 & 113770.90 & 878.9651 & 0.0014 & 0.74 & -0.006 & 16 & 30 & $5 d^{6}$ & $25969.65(4)$ & $139739.81(5)$ & pa \\
\hline 25 & 117 & 880.096 & 113623.98 & 880.0899 & 0.0013 & -0.77 & 0.006 & 15 & 37 & $5 d^{6}$ & $18463.42(5)$ & $132088.18(4)$ & pa \\
\hline 309 & 1285 & 880.187 & 113612.21 & 880.1859 & 0.0019 & -0.15 & 0.001 & 10 & 23 & $5 d^{6}$ & $37387.78(4)$ & $151000.14(5)$ & pa \\
\hline 57 & 235 & 880.715 & 113544.04 & 880.7171 & 0.0015 & 0.21 & -0.002 & 14 & 30 & $5 d^{6}$ & $27670.52(2)$ & $141214.36(3)$ & pa \\
\hline 16 & 47 & 880.922 & 113517.36 & 880.9206 & 0.0015 & -0.25 & 0.002 & 13 & 39 & $5 d^{6}$ & $21190.03(3)$ & $134707.64(2)$ & pa \\
\hline 10 & 645 & 881.354 & 113461.79 & 881.3496 & 0.0021 & -0.56 & 0.004 & 4 & 26 & $5 d^{6}$ & $0.00(4)$ & $113462.35(4)$ & pa \\
\hline 252 & 1835 & 882.228 & 113349.33 & 882.2260 & 0.0015 & -0.31 & 0.002 & 9 & 30 & $5 d^{6}$ & $5178.26(2)$ & $118527.90(2)$ & pa \\
\hline 40 & 145 & 882.319 & 113337.71 & 882.3043 & 0.0016 & -1.87 & 0.015 & 8 & 25 & $5 d^{6}$ & $19128.73(2)$ & $132468.31(2)$ & $\mathrm{pa} *$ \\
\hline 34 & 110 & 882.765 & 113280.48 & 882.7602 & 0.0017 & -0.57 & 0.004 & 15 & 25 & $5 d^{6}$ & $22138.98(2)$ & $135420.03(3)$ & pa \\
\hline 98 & 382 & 883.087 & 113239.13 & 883.0876 & 0.0014 & 0.08 & -0.001 & 13 & 43 & $5 d^{6}$ & $28959.73(3)$ & $142198.78(4)$ & pa \\
\hline 20 & 247 & 884.894 & 113007.94 & 884.8904 & 0.0017 & -0.40 & 0.003 & 8 & 30 & $5 d^{6}$ & $27670.52(2)$ & $140678.86(1)$ & pa \\
\hline 17 & 452 & 886.268 & 112832.62 & 886.2639 & 0.0018 & -0.59 & 0.005 & 8 & 14 & $5 d^{6}$ & $8526.56(1)$ & $121359.77(1)$ & pa \\
\hline 57 & 127 & 886.506 & 112802.34 & 886.5035 & 0.0016 & -0.37 & 0.003 & 10 & 30 & $5 d^{6}$ & $27670.52(2)$ & $140473.23(2)$ & pa \\
\hline 202 & 1600 & 887.281 & 112703.85 & 887.2816 & 0.0014 & 0.07 & -0.001 & 9 & 39 & $5 d^{6}$ & $5824.11(3)$ & $118527.90(2)$ & pa \\
\hline 37 & 622 & 887.521 & 112673.40 & 887.5263 & 0.0024 & 0.68 & -0.005 & 8 & 7 & $5 d^{6}$ & $8687.05(0)$ & $121359.77(1)$ & pa \\
\hline 106 & 550 & 888.204 & 112586.70 & 888.2078 & 0.0014 & 0.43 & -0.003 & 15 & 36 & $5 d^{6}$ & $27940.65(4)$ & $140526.92(3)$ & pa \\
\hline 56 & 817 & 888.839 & 112506.37 & 888.8388 & 0.0013 & 0.03 & 0.000 & 13 & 39 & $5 d^{6}$ & $5824.11(3)$ & $118330.45(3)$ & pa \\
\hline 37 & 212 & 888.966 & 112490.24 & 888.9749 & 0.0015 & 1.13 & -0.009 & 15 & 32 & $5 d^{6}$ & $28652.05(5)$ & $141141.16(4)$ & pa \\
\hline 77 & 417 & 889.181 & 112463.02 & 889.1765 & 0.0015 & -0.60 & 0.005 & 16 & 31 & $5 d^{6}$ & $30693.33(3)$ & $143156.95(4)$ & pa \\
\hline 45 & 687 & 889.957 & 112364.98 & 889.9557 & 0.0018 & -0.16 & 0.001 & 11 & 14 & $5 d^{6}$ & $8526.56(1)$ & $120891.70(2)$ & pa \\
\hline 136 & 392 & 890.288 & 112323.15 & 890.2884 & 0.0017 & 0.00 & 0.000 & 11 & 29 & $5 d^{6}$ & $35440.07(3)$ & $147763.22(4)$ & pa \\
\hline 34 & 62 & 890.670 & 112274.97 & 890.6720 & 0.0030 & 0.19 & -0.001 & 11 & 7 & $5 d^{6}$ & $39120.48(2)$ & $151395.26(3)$ & pa * \\
\hline 92 & 240 & 890.840 & 112253.65 & 890.8319 & 0.0015 & -0.98 & 0.008 & 14 & 43 & $5 d^{6}$ & $28959.73(3)$ & $141214.36(3)$ & pa \\
\hline 9 & 85 & 891.082 & 112223.14 & 891.0942 & 0.0023 & 1.56 & -0.012 & 17 & 12 & $5 d^{6}$ & $33303.67(6)$ & $145525.25(5)$ & pa * \\
\hline 135 & 445 & 891.418 & 112180.83 & 891.4131 & 0.0015 & -0.60 & 0.005 & 15 & 43 & $5 d^{6}$ & $28959.73(3)$ & $141141.16(4)$ & pa \\
\hline 34 & 50 & 891.489 & 112171.95 & 891.4899 & 0.0018 & 0.18 & -0.001 & 13 & 31 & $5 d^{6}$ & $30693.33(3)$ & $142865.10(2)$ & pa \\
\hline 18 & 75 & 891.945 & 112114.60 & 891.9501 & 0.0014 & 0.70 & -0.006 & 11 & 36 & $5 d^{6}$ & $27940.65(4)$ & $140054.55(3)$ & pa* \\
\hline 43 & 35 & 893.639 & 111901.97 & 893.6417 & 0.0020 & 0.30 & -0.002 & 10 & 29 & $5 d^{6}$ & $35440.07(3)$ & $147341.74(3)$ & $\mathrm{pa} *$ \\
\hline 40 & 25 & 894.189 & 111833.14 & 894.1814 & 0.0019 & -0.99 & 0.008 & 12 & 20 & $5 d^{6}$ & $46354.20(4)$ & $158188.33(4)$ & pa * \\
\hline 8 & 77 & 894.466 & 111798.50 & 894.4611 & 0.0013 & -0.66 & 0.005 & 16 & 36 & $5 d^{6}$ & $27940.65(4)$ & $139739.81(5)$ & pa \\
\hline 39 & 122 & 895.003 & 111731.47 & 895.0068 & 0.0016 & 0.48 & -0.004 & 12 & 30 & $5 d^{6}$ & $25969.65(4)$ & $137700.64(3)$ & pa \\
\hline 61 & 330 & 895.127 & 111715.93 & 895.1251 & 0.0015 & -0.30 & 0.002 & 11 & 30 & $5 d^{6}$ & $25969.65(4)$ & $137685.88(5)$ & pa \\
\hline 166 & 332 & 896.464 & 111549.41 & 896.4595 & 0.0020 & -0.53 & 0.004 & 12 & 23 & $5 d^{6}$ & $37387.78(4)$ & $148937.72(5)$ & pa \\
\hline 73 & 677 & 897.374 & 111436.28 & 897.3775 & 0.0014 & 0.46 & -0.004 & 11 & 32 & $5 d^{6}$ & $14546.32(4)$ & $125982.14(5)$ & pa \\
\hline 148 & 170 & 898.817 & 111257.35 & 898.8150 & 0.0023 & -0.25 & 0.002 & 6 & 10 & $5 d^{6}$ & $53982.00(4)$ & $165239.60(5)$ & pa \\
\hline 54 & 237 & 900.968 & 110991.75 & 900.9757 & 0.0018 & 0.97 & -0.008 & 12 & 17 & $5 d^{6}$ & $26238.28(1)$ & $137229.06(2)$ & pa \\
\hline 116 & 910 & 901.728 & 110898.17 & 901.7283 & 0.0013 & 0.02 & 0.000 & 15 & 39 & $5 d^{6}$ & $21190.03(3)$ & $132088.18(4)$ & pa \\
\hline 68 & 120 & 902.963 & 110746.46 & 902.9663 & 0.0019 & 0.35 & -0.003 & 17 & 29 & $5 d^{6}$ & $35440.07(3)$ & $146186.18(3)$ & pa \\
\hline 120 & 1585 & 906.947 & 110260.03 & 906.9496 & 0.0019 & 0.32 & -0.003 & 7 & 14 & $5 d^{6}$ & $8526.56(1)$ & $118786.27(1)$ & pa \\
\hline 48 & 1152 & 908.277 & 110098.52 & 908.2716 & 0.0031 & -0.70 & 0.006 & 7 & 7 & $5 d^{6}$ & $8687.05(0)$ & $118786.27(1)$ & pa \\
\hline 83 & 480 & 908.839 & 110030.43 & 908.8448 & 0.0013 & 0.64 & -0.005 & 14 & 37 & $5 d^{6}$ & $18463.42(5)$ & $128493.21(4)$ & pa \\
\hline 17 & 232 & 909.082 & 110001.10 & 909.0798 & 0.0020 & -0.24 & 0.002 & 9 & 14 & $5 d^{6}$ & $8526.56(1)$ & $118527.90(2)$ & pa \\
\hline
\end{tabular}




\begin{tabular}{|c|c|c|c|c|c|c|c|c|c|c|c|c|c|c|}
\hline 36 & 292 & 909.099 & 109999.06 & 909.0919 & 0.0017 & -0.82 & 0.007 & 16 & 29 & $5 d^{6}$ & $35440.07(3)$ & $145439.95(4)$ & pa * & 100 \\
\hline 11 & 157 & 909.580 & 109940.88 & 909.5767 & 0.0016 & -0.37 & 0.003 & 10 & 25 & $5 d^{6}$ & $19128.73(2)$ & $129069.98(3)$ & pa & \\
\hline 29 & 810 & 910.646 & 109812.19 & 910.6466 & 0.0014 & 0.11 & -0.001 & 8 & 39 & $5 d^{6}$ & $5824.11(3)$ & $115636.19(3)$ & pa & \\
\hline 109 & 580 & 911.194 & 109746.08 & 911.2013 & 0.0014 & 0.85 & -0.007 & 11 & 36 & $5 d^{6}$ & $27940.65(4)$ & $137685.88(5)$ & pa & \\
\hline 66 & 260 & 911.474 & 109712.45 & 911.4760 & 0.0019 & 0.29 & -0.002 & 10 & 17 & $5 d^{6}$ & $26238.28(1)$ & $135950.44(1)$ & pa & \\
\hline 11 & 67 & 912.653 & 109570.71 & 912.6579 & 0.0016 & 0.62 & -0.005 & 10 & 43 & $5 d^{6}$ & $28959.73(3)$ & $138529.81(3)$ & pa * & \\
\hline 131 & 777 & 913.492 & 109470.00 & 913.4993 & 0.0015 & 0.84 & -0.007 & 10 & 32 & $5 d^{6}$ & $28652.05(5)$ & $138121.21(6)$ & pa & \\
\hline 49 & 305 & 913.961 & 109413.91 & 913.9508 & 0.0018 & -1.17 & 0.010 & 15 & 29 & $5 d^{6}$ & $35440.07(3)$ & $144855.15(3)$ & pa * & 100 \\
\hline 47 & 105 & 914.389 & 109362.59 & 914.4010 & 0.0016 & 1.37 & -0.011 & 11 & 31 & $5 d^{6}$ & $30693.33(3)$ & $140054.55(3)$ & pa* & \\
\hline 130 & 790 & 914.854 & 109307.11 & 914.8709 & 0.0016 & 2.06 & -0.017 & 15 & 31 & $5 d^{6}$ & $30693.33(3)$ & $139998.38(4)$ & pa* & bl \\
\hline 119 & 437 & 919.072 & 108805.36 & 919.0635 & 0.0020 & -1.05 & 0.009 & 12 & 20 & $5 d^{6}$ & $46354.20(4)$ & $155160.61(5)$ & $\mathrm{pa} *$ & \\
\hline 16 & 17 & 919.617 & 108740.91 & 919.6171 & 0.0017 & 0.00 & 0.000 & 12 & 43 & $5 d^{6}$ & $28959.73(3)$ & $137700.64(3)$ & pa * & \\
\hline 152 & 205 & 920.205 & 108671.46 & 920.2007 & 0.0023 & -0.48 & 0.004 & 11 & 10 & $5 d^{6}$ & $53982.00(4)$ & $162653.94(5)$ & pa & \\
\hline 47 & 397 & 920.338 & 108655.67 & 920.3379 & 0.0014 & -0.07 & 0.001 & 13 & 32 & $5 d^{6}$ & $14546.32(4)$ & $123202.06(3)$ & pa & \\
\hline 18 & 550 & 920.562 & 108629.28 & 920.5632 & 0.0015 & 0.13 & -0.001 & 8 & 30 & $5 d^{6}$ & $5178.26(2)$ & $113807.41(2)$ & pa & \\
\hline 32 & 125 & 922.518 & 108399.00 & 922.5214 & 0.0034 & 0.43 & -0.004 & 13 & 8 & $5 d^{6}$ & $34466.53(1)$ & $142865.10(2)$ & pa & \\
\hline 46 & 112 & 923.535 & 108279.62 & 923.5323 & 0.0016 & -0.30 & 0.003 & 10 & 30 & $5 d^{6}$ & $27670.52(2)$ & $135950.44(1)$ & pa & \\
\hline 15 & 82 & 923.610 & 108270.85 & 923.6226 & 0.0016 & 1.52 & -0.013 & 12 & 43 & $5 d^{6}$ & $28959.73(3)$ & $137229.06(2)$ & pa * & \\
\hline 57 & 252 & 924.745 & 108137.86 & 924.7488 & 0.0019 & 0.39 & -0.003 & 17 & 23 & $5 d^{6}$ & $37387.78(4)$ & $145525.25(5)$ & pa & \\
\hline 18 & 32 & 927.522 & 107814.13 & 927.5230 & 0.0019 & 0.09 & -0.001 & 6 & 30 & $5 d^{6}$ & $27670.52(2)$ & $135484.56(1)$ & pa & \\
\hline 95 & 150 & 927.653 & 107798.99 & 927.6580 & 0.0022 & 0.64 & -0.005 & 13 & 20 & $5 d^{6}$ & $46354.20(4)$ & $154152.55(3)$ & pa & \\
\hline 76 & 460 & 928.357 & 107717.24 & 928.3596 & 0.0017 & 0.36 & -0.003 & 16 & 29 & $5 d^{6}$ & $35440.07(3)$ & $143156.95(4)$ & pa & \\
\hline 7 & 375 & 929.034 & 107638.66 & 929.0379 & 0.0022 & 0.42 & -0.004 & 4 & 39 & $5 d^{6}$ & $5824.11(3)$ & $113462.35(4)$ & pa & \\
\hline 22 & 115 & 930.890 & 107424.11 & 930.8818 & 0.0021 & -0.92 & 0.008 & 13 & 29 & $5 d^{6}$ & $35440.07(3)$ & $142865.10(2)$ & pa * & \\
\hline 25 & 255 & 931.471 & 107357.03 & 931.4806 & 0.0015 & 1.06 & -0.009 & 14 & 32 & $5 d^{6}$ & $14546.32(4)$ & $121902.29(3)$ & pa & \\
\hline 61 & 1160 & 931.937 & 107303.38 & 931.9388 & 0.0013 & 0.20 & -0.002 & 14 & 39 & $5 d^{6}$ & $21190.03(3)$ & $128493.21(4)$ & pa & \\
\hline 23 & 205 & 933.499 & 107123.83 & 933.4918 & 0.0019 & -0.84 & 0.007 & 14 & 23 & $5 d^{6}$ & $37387.78(4)$ & $144512.45(5)$ & pa* & \\
\hline 52 & 275 & 934.246 & 107038.24 & 934.2553 & 0.0017 & 1.11 & -0.010 & 13 & 30 & $5 d^{6}$ & $27670.52(2)$ & $134707.64(2)$ & pa & \\
\hline 55 & 387 & 935.573 & 106886.43 & 935.5828 & 0.0017 & 1.18 & -0.010 & 11 & 23 & $5 d^{6}$ & $19096.89(6)$ & $125982.14(5)$ & pa & \\
\hline 76 & 647 & 936.664 & 106761.89 & 936.6682 & 0.0014 & 0.51 & -0.004 & 15 & 37 & $5 d^{6}$ & $18463.42(5)$ & $125224.81(4)$ & pa & \\
\hline 25 & 10 & 937.835 & 106628.52 & 937.8352 & 0.0031 & -0.02 & 0.000 & 9 & 7 & $5 d^{6}$ & $46564.59(2)$ & $153193.13(2)$ & pa* & \\
\hline 12 & 22 & 942.645 & 106084.44 & 942.6434 & 0.0015 & -0.22 & 0.002 & 16 & 36 & $5 d^{6}$ & $27940.65(4)$ & $134025.31(4)$ & pa* & \\
\hline 66 & 167 & 943.834 & 105950.80 & 943.8392 & 0.0019 & 0.55 & -0.005 & 15 & 20 & $5 d^{6}$ & $46354.20(4)$ & $152304.45(5)$ & pa & \\
\hline 14 & 32 & 945.449 & 105769.82 & 945.4551 & 0.0020 & 0.65 & -0.006 & 16 & 23 & $5 d^{6}$ & $37387.78(4)$ & $143156.95(4)$ & $\mathrm{pa} *$ & \\
\hline 28 & 117 & 949.051 & 105368.46 & 949.0610 & 0.0016 & 1.15 & -0.010 & 14 & 30 & $5 d^{6}$ & $27670.52(2)$ & $133037.83(3)$ & pa* & \\
\hline 29 & 747 & 954.219 & 104797.76 & 954.2186 & 0.0017 & -0.03 & 0.000 & 8 & 30 & $5 d^{6}$ & $27670.52(2)$ & $132468.31(2)$ & pa * & 200, Ir III \\
\hline 20 & 32 & 955.694 & 104635.95 & 955.6853 & 0.0022 & -1.01 & 0.009 & 10 & 17 & $5 d^{6}$ & $26238.28(1)$ & $130875.24(2)$ & $\mathrm{pa} *$ & \\
\hline 33 & 37 & 959.974 & 104169.53 & 959.9754 & 0.0020 & 0.19 & -0.002 & 11 & 16 & $5 d^{6}$ & $52259.88(3)$ & $156429.22(2)$ & pa* & \\
\hline 30 & 915 & 960.158 & 104149.55 & 960.1740 & 0.0017 & 1.76 & -0.016 & 6 & 37 & $5 d^{6}$ & $18463.42(5)$ & $122611.21(6)$ & pa * & 300 \\
\hline 20 & 140 & 963.533 & 103784.68 & 963.5385 & 0.0015 & 0.55 & -0.005 & 13 & 32 & $5 d^{6}$ & $14546.32(4)$ & $118330.45(3)$ & pa & \\
\hline 40 & 332 & 965.084 & 103617.92 & 965.0874 & 0.0017 & 0.36 & -0.003 & 9 & 25 & $5 d^{6}$ & $19128.73(2)$ & $122746.29(2)$ & pa & \\
\hline 25 & 625 & 966.044 & 103515.00 & 966.0499 & 0.0020 & 0.68 & -0.006 & 6 & 23 & $5 d^{6}$ & $19096.89(6)$ & $122611.21(6)$ & pa * & 300 \\
\hline 78 & 65 & 966.190 & 103499.27 & 966.1924 & 0.0024 & 0.22 & -0.002 & 12 & 10 & $5 d^{6}$ & $53982.00(4)$ & $157481.05(5)$ & pa & \\
\hline 28 & 110 & 966.774 & 103436.81 & 966.7802 & 0.0016 & 0.68 & -0.006 & 15 & 32 & $5 d^{6}$ & $28652.05(5)$ & $132088.18(4)$ & pa & \\
\hline
\end{tabular}




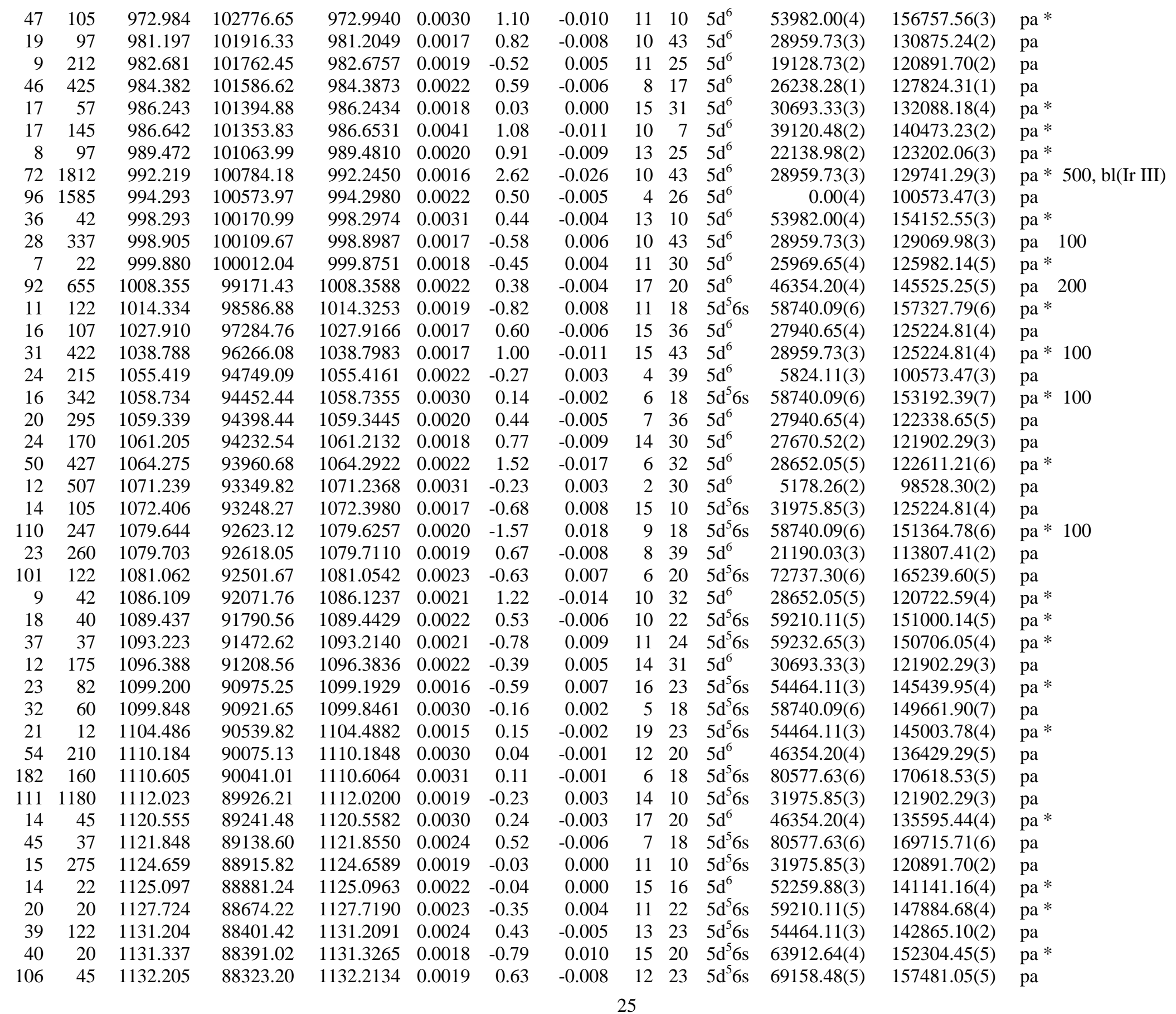




$\begin{array}{rrr}16 & 7 & 1133.625 \\ 25 & 27 & 1134.506 \\ 166 & 297 & 1134.939 \\ 19 & 412 & 1136.801 \\ 19 & 77 & 1139.460 \\ 11 & 30 & 1139.795 \\ 95 & 345 & 1143.080 \\ 42 & 10 & 1145.448 \\ 97 & 237 & 1147.572 \\ 42 & 35 & 1148.261 \\ 48 & 112 & 1149.092 \\ 19 & 15 & 1149.708 \\ 47 & 67 & 1149.930 \\ 20 & 25 & 1150.045 \\ 78 & 40 & 1150.155 \\ 90 & 22 & 1150.479 \\ 242 & 695 & 1152.275 \\ 11 & 30 & 1153.077 \\ 10 & 37 & 1153.713 \\ 19 & 27 & 1154.784 \\ 129 & 435 & 1155.374 \\ 18 & 20 & 1156.469 \\ 299 & 997 & 1158.017 \\ 56 & 70 & 1158.543 \\ 25 & 45 & 1158.692 \\ 129 & 160 & 1159.695 \\ 50 & 74 & 1161.036 \\ 85 & 82 & 1165.594 \\ 25 & 50 & 1165.869 \\ 74 & 93 & 1165.896 \\ 58 & 42 & 1167.004 \\ 41 & 40 & 1167.257 \\ 66 & 35 & 1167.571 \\ 43 & 40 & 1168.414 \\ 145 & 261 & 1169.124 \\ 19 & 63 & 1170.698 \\ 41 & 78 & 1176.599 \\ 85 & 69 & 1177.183 \\ 156 & 34 & 1177.802 \\ 69 & 63 & 1177.874 \\ 95 & 130 & 1181.065 \\ 172 & 113 & 1181.410 \\ 22 & 30 & 1182.693 \\ 134 & 879 & 1183.128\end{array}$

$\begin{array}{lllll}88212.63 & 1133.6153 & 0.0033 & -0.72\end{array}$ $\begin{array}{lllll}88144.08 & 1134.5046 & 0.0025 & -0.12\end{array}$ $\begin{array}{llll}88110.45 & 1134.9446 & 0.0024 & 0.42\end{array}$ $\begin{array}{llll}87966.14 & 1136.8071 & 0.0023 & 0.47\end{array}$ $\begin{array}{lllll}87760.90 & 1139.4685 & 0.0060 & 0.69\end{array}$ $\begin{array}{lllll}87735.08 & 1139.8003 & 0.0017 & 0.41\end{array}$ $\begin{array}{lllll}87482.92 & 1143.0757 & 0.0018 & -0.35\end{array}$ $\begin{array}{lllll}87302.12 & 1145.4495 & 0.0020 & 0.15\end{array}$ $\begin{array}{lllll}87140.48 & 1147.5783 & 0.0030 & 0.45\end{array}$ $\begin{array}{llll}87088.21 & 1148.2704 & 0.0023 & 0.71\end{array}$ $\begin{array}{lllll}87025.25 & 1149.0897 & 0.0030 & -0.16\end{array}$ $\begin{array}{lllll}86978.61 & 1149.7003 & 0.0025 & -0.58\end{array}$ $\begin{array}{lllll}86961.85 & 1149.9302 & 0.0016 & 0.05\end{array}$ $\begin{array}{lllll}86953.11 & 1150.0396 & 0.0024 & -0.42\end{array}$ $\begin{array}{lllll}86944.83 & 1150.1468 & 0.0031 & -0.59\end{array}$ $\begin{array}{llll}86920.35 & 1150.4768 & 0.0040 & -0.13\end{array}$ $\begin{array}{lllll}86784.86 & 1152.2707 & 0.0019 & -0.30\end{array}$ $\begin{array}{lllll}86724.47 & 1153.0734 & 0.0031 & -0.28\end{array}$ $\begin{array}{lllll}86676.66 & 1153.7079 & 0.0019 & -0.39\end{array}$ $\begin{array}{lllll}86596.25 & 1154.7780 & 0.0051 & -0.48\end{array}$ $\begin{array}{llll}86552.02 & 1155.3741 & 0.0022 & -0.03\end{array}$ $\begin{array}{lllll}86470.08 & 1156.4642 & 0.0018 & -0.39\end{array}$ $\begin{array}{lllll}86354.49 & 1158.0159 & 0.0019 & -0.11\end{array}$ $\begin{array}{lllll}86315.29 & 1158.5453 & 0.0018 & 0.15\end{array}$ $\begin{array}{lllll}86304.21 & 1158.6943 & 0.0023 & 0.17\end{array}$ $\begin{array}{lllll}86229.57 & 1159.6914 & 0.0018 & -0.27\end{array}$ $\begin{array}{lllll}86129.97 & 1161.0294 & 0.0031 & -0.49\end{array}$ $\begin{array}{lllll}85793.13 & 1165.5872 & 0.0017 & -0.54\end{array}$ $\begin{array}{llll}85772.96 & 1165.8767 & 0.0022 & 0.60\end{array}$ $\begin{array}{lllll}85770.98 & 1165.8935 & 0.0016 & -0.15\end{array}$ $\begin{array}{lllll}85689.53 & 1166.9991 & 0.0019 & -0.34\end{array}$ $\begin{array}{lllll}85670.95 & 1167.2507 & 0.0024 & -0.45\end{array}$ $\begin{array}{lllll}85647.93 & 1167.5674 & 0.0019 & -0.23\end{array}$ $\begin{array}{lllll}85586.10 & 1168.4064 & 0.0021 & -0.56\end{array}$ $\begin{array}{lllll}85534.11 & 1169.1220 & 0.0017 & -0.16\end{array}$ $\begin{array}{lllll}85419.13 & 1170.6975 & 0.0024 & -0.03\end{array}$ $\begin{array}{lllll}84990.75 & 1176.5846 & 0.0021 & -1.01\end{array}$ $\begin{array}{llll}84948.54 & 1177.1847 & 0.0040 & 0.10\end{array}$ $\begin{array}{lllll}84903.91 & 1177.8030 & 0.0030 & 0.07\end{array}$ $\begin{array}{lllll}84898.73 & 1177.8671 & 0.0019 & -0.49\end{array}$ $\begin{array}{lllll}84669.35 & 1181.0559 & 0.0023 & -0.65\end{array}$ $\begin{array}{llll}84644.62 & 1181.4110 & 0.0040 & 0.07\end{array}$ $\begin{array}{lllll}84552.82 & 1182.6860 & 0.0061 & -0.48\end{array}$ $\begin{array}{lllll}84521.67 & 1183.1276 & 0.0019 & -0.07\end{array}$ $\begin{array}{llllll}0.009 & 10 & 16 & 5 \mathrm{~d}^{6} & 52259.88(3) & 140473.23(2)\end{array}$ ра * $\begin{array}{lllllll}0.002 & 17 & 22 & 5 \mathrm{~d}^{5} 6 \mathrm{~s} & 58041.98(4) & 146186.18(3) & \text { pa } *\end{array}$ $\begin{array}{lllllll}-0.005 & 7 & 20 & 5 d^{5} 6 s & 81605.68(5) & 169715.71(6) & \text { pa }\end{array}$ $\begin{array}{lllllll}-0.006 & 8 & 30 & 5 d^{6} & 27670.52(2) & 115636.19(3) & \text { pa } * \text { bl(Al IV) }\end{array}$ $\begin{array}{lllllll}-0.009 & 12 & 6 & 5 d^{5} 6 s & 67091.85(1) & 154852.06(2) & \text { pa* }\end{array}$ $\begin{array}{llllll}-0.005 & 13 & 23 & 5 d^{5} 6 s & 54464.11(3) & 142198.78(4)\end{array}$ ра* $\begin{array}{lllllll}0.005 & 17 & 22 & 5 \mathrm{~d}^{5} 6 \mathrm{~s} & 58041.98(4) & 145525.25(5) & \text { pa }\end{array}$ $\begin{array}{lllllll}-0.002 & 14 & 25 & 5 d^{5} 6 s & 66110.16(3) & 153412.13(4) & \text { pa* }\end{array}$ $\begin{array}{lllllll}-0.006 & 7 & 19 & 5 d^{5} 6 s & 73067.88(5) & 160207.91(4) & \text { pa }\end{array}$ $\begin{array}{lllllll}-0.009 & 10 & 20 & 5 d^{5} 6 s & 63912.64(4) & 151000.14(5) & \text { pa }\end{array}$ $\begin{array}{lllllll}0.002 & 8 & 20 & 5 d^{5} 6 s & 72737.30(6) & 159762.71(5) & \text { pa } *\end{array}$ $\begin{array}{lllllll}0.008 & 17 & 21 & 5 d^{5} 6 s & 59206.99(2) & 146186.18(3) & \text { ра } *\end{array}$ $\begin{array}{llllll}-0.001 & 19 & 22 & 5 d^{5} 6 s & 58041.98(4) & 145003.78(4)\end{array}$ $\begin{array}{llllll}0.006 & 17 & 24 & 5 d^{5} 6 s & 59232.65(3) & 146186.18(3)\end{array}$ pa* $\begin{array}{lllllll}0.008 & 7 & 22 & 5 d^{5} 6 s & 76295.33(5) & 163240.75(5) & \text { pa }\end{array}$ $\begin{array}{lllllll}0.002 & 3 & 18 & 5 \mathrm{~d}^{5} 6 \mathrm{~s} & 80577.63(6) & 167498.11(7) & \text { pa }\end{array}$ $\begin{array}{lllllll}0.004 & 17 & 18 & 5 \mathrm{~d}^{5} 6 \mathrm{~s} & 58740.09(6) & 145525.25(5) & \text { pa }\end{array}$ $0.004 \quad 8 \quad 14 \quad 5 d^{5} 6 s \quad 50417.92(2) \quad 137142.67(1) \quad$ pa* $\begin{array}{llllll}0.005 & 15 & 23 & 5 \mathrm{~d}^{5} 6 \mathrm{~s} & 54464.11(3) & 141141.16(4)\end{array}$ ра $*$ $\begin{array}{lllllll}0.006 & 12 & 19 & 5 \mathrm{~d}^{5} 6 \mathrm{~s} & 68255.33(2) & 154852.06(2) & \text { pa* }\end{array}$ $\begin{array}{lllllll}0.000 & 9 & 10 & 5 d^{5} 6 s & 31975.85(3) & 118527.90(2) & \text { рa }\end{array}$ $\begin{array}{lllllll}0.005 & 14 & 22 & 5 \mathrm{~d}^{5} 6 \mathrm{~s} & 58041.98(4) & 144512.45(5) & \text { pa* }\end{array}$ $\begin{array}{lllllll}0.002 & 13 & 10 & 5 d^{5} 6 s & 31975.85(3) & 118330.45(3) & \text { pa }\end{array}$ $\begin{array}{lllllll}-0.002 & 17 & 22 & 5 d^{5} 6 s & 59210.11(5) & 145525.25(5) & \text { pa }\end{array}$ $\begin{array}{lllllll}-0.002 & 7 & 24 & 5 d^{5} 6 s & 59232.65(3) & 145536.69(3) & \text { pa }\end{array}$ $\begin{array}{lllllll}0.004 & 16 & 22 & 5 \mathrm{~d}^{5} 6 \mathrm{~s} & 59210.11(5) & 145439.95(4) & \mathrm{pb}\end{array}$ $\begin{array}{lllllll}0.007 & 11 & 18 & 5 \mathrm{~d}^{5} 6 \mathrm{~s} & 70298.76(2) & 156429.22(2) & \mathrm{pb}\end{array}$ $\begin{array}{lllllll}0.007 & 19 & 22 & 5 \mathrm{~d}^{5} 6 \mathrm{~s} & 59210.11(5) & 145003.78(4) & \mathrm{pb}\end{array}$ $\begin{array}{lllllll}-0.008 & 14 & 18 & 5 \mathrm{~d}^{5} 6 \mathrm{~s} & 58740.09(6) & 144512.45(5) & \mathrm{pb} *\end{array}$ $\begin{array}{lllllll}0.002 & 19 & 24 & 5 \mathrm{~d}^{5} 6 \mathrm{~s} & 59232.65(3) & 145003.78(4) & \mathrm{pb}\end{array}$ $\begin{array}{lllllll}0.005 & 14 & 22 & 5 \mathrm{~d}^{5} 6 \mathrm{~s} & 58041.98(4) & 143731.85(5) & \mathrm{pb}\end{array}$ $\begin{array}{llllllll}0.006 & 10 & 7 & 5 d^{6} & 52858.41(2) & 138529.81(3) & \text { pb }\end{array}$ $\begin{array}{lllllll}0.003 & 15 & 21 & 5 \mathrm{~d}^{5} 6 \mathrm{~s} & 59206.99(2) & 144855.15(3) & \mathrm{pb}\end{array}$ $\begin{array}{lllllll}0.008 & 14 & 23 & 5 \mathrm{~d}^{5} 6 \mathrm{~s} & 55627.70(2) & 141214.36(3) & \mathrm{pb}\end{array}$ $\begin{array}{lllllll}0.002 & 15 & 23 & 5 \mathrm{~d}^{5} 6 \mathrm{~s} & 54464.11(3) & 139998.38(4) & \mathrm{pb}\end{array}$ $\begin{array}{lllllll}0.000 & 9 & 21 & 5 \mathrm{~d}^{5} 6 \mathrm{~s} & 59206.99(2) & 144626.15(2) & \mathrm{pb} *\end{array}$ $\begin{array}{lllllll}0.014 & 14 & 18 & 5 \mathrm{~d}^{5} 6 \mathrm{~s} & 58740.09(6) & 143731.85(5) & \mathrm{pb} *\end{array}$ $\begin{array}{lllllll}-0.001 & 9 & 16 & 5 d^{5} 6 \mathrm{~s} & 84734.80(4) & 169683.24(3) & \mathrm{pb}\end{array}$ $\begin{array}{lllllll}-0.001 & 4 & 13 & 5 d^{5} 6 s & 90123.22(5) & 175027.06(4) & \text { pb }\end{array}$ $\begin{array}{lllllll}0.007 & 15 & 23 & 5 d^{5} 6 s & 55627.70(2) & 140526.92(3) & \text { pb }\end{array}$ $\begin{array}{lllllll}0.009 & 12 & 23 & 5 d^{5} 6 s & 69158.48(5) & 153828.48(4) & \text { pb }\end{array}$ $\begin{array}{lllllll}-0.001 & 2 & 19 & 5 d^{5} 6 s & 74545.24(3) & 159189.79(3) & p b\end{array}$ $\begin{array}{lllllll}0.007 & 12 & 18 & 5 \mathrm{~d}^{5} 6 \mathrm{~s} & 70298.76(2) & 154852.06(2) & \mathrm{pb} *\end{array}$

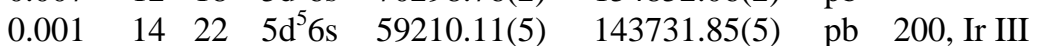




$\begin{array}{rrr}33 & 373 & 1183.390 \\ 142 & 77 & 1184.653 \\ 53 & 40 & 1188.253 \\ 67 & 65 & 1190.526 \\ 174 & 214 & 1191.583 \\ 125 & 54 & 1192.980 \\ 118 & 465 & 1194.695 \\ 175 & 1176 & 1195.304 \\ 79 & 40 & 1195.556 \\ 122 & 94 & 1197.199 \\ 123 & 109 & 1198.291 \\ 48 & 88 & 1199.964 \\ 279 & 387 & 1202.314 \\ 140 & 82 & 1202.793 \\ 154 & 129 & 1204.912 \\ 130 & 24 & 1206.868 \\ 66 & 65 & 1210.356 \\ 64 & 21 & 1211.325 \\ 27 & 134 & 1212.895 \\ 166 & 61 & 1213.588 \\ 161 & 104 & 1213.717 \\ 69 & 31 & 1213.826 \\ 161 & 428 & 1216.240 \\ 146 & 114 & 1216.364 \\ 163 & 66 & 1218.132 \\ 273 & 160 & 1218.388 \\ 112 & 139 & 1218.423 \\ 174 & 295 & 1219.325 \\ 174 & 144 & 1219.782 \\ 59 & 77 & 1220.162 \\ 100 & 149 & 1220.543 \\ 211 & 296 & 1220.877 \\ 73 & 26 & 1220.988 \\ 70 & 29 & 1221.245 \\ 155 & 112 & 1221.798 \\ 319 & 322 & 1221.991 \\ 29 & 330 & 1222.018 \\ 61 & 53 & 1222.872 \\ 51 & 244 & 1223.009 \\ 107 & 28 & 1223.859 \\ 308 & 623 & 1224.024 \\ 126 & 112 & 1224.686 \\ 311 & 226 & 1225.300 \\ 153 & 150 & 1225.466\end{array}$

$\begin{array}{llll}84503.00 & 1183.3953 & 0.0031 & 0.38\end{array}$ $\begin{array}{lllll}84412.88 & 1184.6493 & 0.0020 & -0.29\end{array}$ $\begin{array}{llll}84157.19 & 1188.2581 & 0.0018 & 0.39\end{array}$ $\begin{array}{lllll}83996.49 & 1190.5245 & 0.0043 & -0.10\end{array}$ $\begin{array}{lllll}83922.00 & 1191.5808 & 0.0021 & -0.13\end{array}$ $\begin{array}{lllll}83823.70 & 1192.9787 & 0.0024 & -0.09\end{array}$ $\begin{array}{lllll}83703.37 & 1194.6916 & 0.0031 & -0.24\end{array}$ $\begin{array}{llll}83660.70 & 1195.3095 & 0.0022 & 0.36\end{array}$ $\begin{array}{llll}83643.09 & 1195.5560 & 0.0024 & 0.00\end{array}$ $\begin{array}{lllll}83528.32 & 1197.1969 & 0.0040 & -0.12\end{array}$ $\begin{array}{llll}83452.18 & 1198.2921 & 0.0020 & 0.07\end{array}$ $\begin{array}{llll}83335.81 & 1199.9679 & 0.0022 & 0.25\end{array}$ $\begin{array}{lllll}83172.96 & 1202.3222 & 0.0022 & 0.58\end{array}$ $\begin{array}{llll}83139.82 & 1202.7915 & 0.0024 & -0.11\end{array}$ $\begin{array}{lllll}82993.65 & 1204.9116 & 0.0050 & 0.01\end{array}$ $\begin{array}{llll}82859.08 & 1206.8741 & 0.0064 & 0.39\end{array}$ $\begin{array}{lllll}82620.31 & 1210.3620 & 0.0020 & 0.40\end{array}$ $\begin{array}{llll}82554.26 & 1211.3232 & 0.0061 & -0.09\end{array}$ $\begin{array}{llll}82447.38 & 1212.8892 & 0.0024 & -0.38\end{array}$ $\begin{array}{lllll}82400.31 & 1213.5921 & 0.0030 & 0.30\end{array}$ $\begin{array}{llll}82391.52 & 1213.7180 & 0.0030 & 0.06\end{array}$ $\begin{array}{lllll}82384.15 & 1213.8337 & 0.0050 & 0.54\end{array}$ $\begin{array}{lllll}82220.59 & 1216.2415 & 0.0021 & 0.08\end{array}$ $\begin{array}{lllll}82212.21 & 1216.3627 & 0.0024 & -0.11\end{array}$ $\begin{array}{lllll}82092.89 & 1218.1347 & 0.0023 & 0.16\end{array}$ $\begin{array}{lllll}82075.66 & 1218.3784 & 0.0024 & -0.65\end{array}$ $\begin{array}{lllll}82073.31 & 1218.4284 & 0.0022 & 0.37\end{array}$ $\begin{array}{llll}82012.58 & 1219.3253 & 0.0019 & 0.01\end{array}$ $\begin{array}{llll}81981.85 & 1219.7843 & 0.0022 & 0.14\end{array}$ $\begin{array}{lllll}81956.36 & 1220.1610 & 0.0019 & -0.04\end{array}$ $\begin{array}{lllll}81930.75 & 1220.5385 & 0.0021 & -0.30\end{array}$ $\begin{array}{lllll}81908.30 & 1220.8744 & 0.0020 & -0.21\end{array}$ $\begin{array}{llll}81900.86 & 1220.9923 & 0.0041 & 0.26\end{array}$ $\begin{array}{lllll}81883.62 & 1221.2401 & 0.0030 & -0.36\end{array}$ $\begin{array}{lllll}81846.57 & 1221.7967 & 0.0050 & -0.10\end{array}$ $\begin{array}{lllll}81833.66 & 1221.9913 & 0.0030 & 0.02\end{array}$ $\begin{array}{llll}81831.84 & 1222.0224 & 0.0022 & 0.28\end{array}$ $\begin{array}{llll}81774.71 & 1222.8748 & 0.0025 & 0.19\end{array}$ $\begin{array}{lllll}81765.55 & 1223.0108 & 0.0021 & 0.12\end{array}$ $\begin{array}{llll}81708.75 & 1223.8726 & 0.0030 & 0.90\end{array}$ $\begin{array}{lllll}81697.73 & 1224.0227 & 0.0018 & -0.10\end{array}$ $\begin{array}{llll}81653.55 & 1224.6938 & 0.0023 & 0.49\end{array}$ $\begin{array}{lllll}81612.69 & 1225.3008 & 0.0020 & 0.08\end{array}$ $\begin{array}{lllll}81601.59 & 1225.4698 & 0.0020 & 0.23\end{array}$

\begin{tabular}{|c|c|c|c|c|c|}
\hline 0.005 & 4 & 43 & $5 d^{6}$ & 28959.73(3) & $113462.35(4)$ \\
\hline 0.004 & 12 & 19 & $5 d^{5} 6 s$ & $73067.88(5)$ & $157481.05(5)$ \\
\hline-0.006 & 13 & 22 & $5 d^{5} 6 s$ & $58041.98(4)$ & $142198.78(4)$ \\
\hline 0.001 & 8 & 15 & $5 d^{5} 6 s$ & $75164.22(2)$ & $159160.81(2)$ \\
\hline 0.002 & 10 & 18 & $5 d^{5} 6 s$ & $58740.09(6)$ & $142662.22(6)$ \\
\hline 0.001 & 8 & 23 & $5 d^{5} 6 s$ & $69158.48(5)$ & $152982.27(4)$ \\
\hline 0.003 & 8 & 19 & $5 d^{5} 6 s$ & $79502.00(4)$ & $163205.61(3)$ \\
\hline-0.005 & 8 & 10 & $5 d^{5} 6 s$ & $31975.85(3)$ & $115636.19(3)$ \\
\hline 0.000 & 12 & 19 & $5 d^{5} 6 s$ & $74545.24(3)$ & $158188.33(4)$ \\
\hline 0.002 & 4 & 5 & $5 d^{5} 6 s$ & $65108.69(0)$ & $148637.14(1)$ \\
\hline-0.001 & 10 & 22 & $5 d^{5} 6 s$ & $59210.11(5)$ & $142662.22(6)$ \\
\hline-0.004 & 17 & 16 & $5 d^{6}$ & $52259.88(3)$ & $135595.44(4)$ \\
\hline-0.008 & 14 & 22 & $5 d^{5} 6 s$ & $58041.98(4)$ & $141214.36(3)$ \\
\hline 0.002 & 11 & 19 & $5 d^{5} 6 s$ & $68255.33(2)$ & $151395.26(3)$ \\
\hline 0.000 & 2 & 19 & $5 d^{5} 6 s$ & $68255.33(2)$ & $151248.97(2)$ \\
\hline-0.006 & 7 & 14 & $5 d^{5} 6 s$ & $82458.31(3)$ & $165317.00(2)$ \\
\hline-0.006 & 14 & 14 & $5 d^{5} 6 s$ & $50417.92(2)$ & $133037.83(3)$ \\
\hline 0.001 & 5 & 19 & $5 d^{5} 6 s$ & $74545.24(3)$ & $157099.59(2)$ \\
\hline 0.006 & 13 & 16 & $5 d^{6}$ & $52259.88(3)$ & $134707.64(2)$ \\
\hline-0.004 & 9 & 19 & $5 d^{5} 6 s$ & $79502.00(4)$ & $161902.01(4)$ \\
\hline-0.001 & 8 & 20 & $5 d^{5} 6 s$ & $81605.68(5)$ & $163997.14(4)$ \\
\hline-0.008 & 8 & 9 & $5 d^{5} 6 s$ & $81351.39(2)$ & $163735.00(3)$ \\
\hline-0.001 & 14 & 26 & $5 d^{5} 6 s$ & $71191.62(4)$ & $153412.13(4)$ \\
\hline 0.002 & 11 & 19 & $5 d^{5} 6 s$ & $74545.24(3)$ & $156757.56(3)$ \\
\hline-0.002 & 12 & 19 & $5 d^{5} 6 s$ & $73067.88(5)$ & $155160.61(5)$ \\
\hline 0.010 & 11 & 18 & $5 d^{5} 6 s$ & $80577.63(6)$ & $162653.94(5)$ \\
\hline-0.005 & 12 & 23 & $5 d^{5} 6 s$ & $55627.70(2)$ & $137700.64(3)$ \\
\hline 0.000 & 11 & 22 & $5 d^{5} 6 s$ & $58041.98(4)$ & $140054.55(3)$ \\
\hline-0.002 & 14 & 24 & $5 d^{5} 6 s$ & $59232.65(3)$ & $141214.36(3)$ \\
\hline 0.001 & 15 & 22 & $5 d^{5} 6 s$ & $58041.98(4)$ & $139998.38(4)$ \\
\hline 0.004 & 15 & 22 & $5 d^{5} 6 s$ & $59210.11(5)$ & $141141.16(4)$ \\
\hline 0.003 & 15 & 24 & $5 d^{5} 6 s$ & $59232.65(3)$ & $141141.16(4)$ \\
\hline-0.004 & 9 & 17 & $5 d^{5} 6 s$ & $87782.64(4)$ & $169683.24(3)$ \\
\hline 0.005 & 11 & 19 & $5 d^{5} 6 s$ & $74545.24(3)$ & $156429.22(2)$ \\
\hline 0.002 & 2 & 6 & $5 d^{5} 6 s$ & $71884.65(1)$ & $153731.32(1)$ \\
\hline 0.000 & 11 & 11 & $5 d^{5} 6 s$ & $75494.15(7)$ & $157327.79(6)$ \\
\hline-0.004 & 8 & 10 & $5 d^{5} 6 s$ & $31975.85(3)$ & $113807.41(2)$ \\
\hline-0.003 & 11 & 25 & $5 d^{5} 6 s$ & $66110.16(3)$ & $147884.68(4)$ \\
\hline-0.002 & 16 & 16 & $5 d^{6}$ & $52259.88(3)$ & $134025.31(4)$ \\
\hline-0.014 & 8 & 17 & $5 d^{5} 6 s$ & $87782.64(4)$ & $169490.49(5)$ \\
\hline 0.001 & 16 & 22 & $5 d^{5} 6 s$ & $58041.98(4)$ & $139739.81(5)$ \\
\hline-0.007 & 11 & 25 & $5 d^{5} 6 s$ & $66110.16(3)$ & $147763.22(4)$ \\
\hline-0.001 & 17 & 20 & $5 d^{5} 6 s$ & $63912.64(4)$ & $145525.25(5)$ \\
\hline-0.003 & 12 & 23 & $5 d^{5} 6 s$ & $55627.70(2)$ & $137229.06(2)$ \\
\hline
\end{tabular}




$\begin{array}{rrr}121 & 770 & 1225.562 \\ 166 & 62 & 1226.143 \\ 297 & 553 & 1226.582 \\ 62 & 49 & 1226.769 \\ 1887 & 1349 & 1227.202 \\ 65 & 455 & 1227.420 \\ 108 & 138 & 1230.100 \\ 45 & 64 & 1230.525 \\ 295 & 598 & 1230.914 \\ 234 & 166 & 1231.044 \\ 141 & 37 & 1231.136 \\ 348 & 741 & 1231.619 \\ 181 & 44 & 1231.748 \\ 330 & 884 & 1232.573 \\ 175 & 64 & 1232.849 \\ 193 & 48 & 1232.997 \\ 247 & 141 & 1233.830 \\ 124 & 44 & 1233.898 \\ 68 & 51 & 1234.081 \\ 52 & 74 & 1234.151 \\ 106 & 134 & 1234.570 \\ 207 & 162 & 1235.440 \\ 97 & 32 & 1235.749 \\ 158 & 72 & 1236.523 \\ 48 & 37 & 1236.888 \\ 50 & 82 & 1237.961 \\ 396 & 761 & 1238.146 \\ 284 & 136 & 1238.217 \\ 208 & 231 & 1240.696 \\ 348 & 904 & 1241.775 \\ 142 & 33 & 1242.161 \\ 147 & 111 & 1242.326 \\ 300 & 244 & 1242.929 \\ 94 & 56 & 1243.010 \\ 195 & 803 & 1243.687 \\ 86 & 40 & 1244.483 \\ 125 & 24 & 1244.826 \\ 43 & 24 & 1244.983 \\ 61 & 55 & 1245.889 \\ 113 & 41 & 1245.940 \\ 203 & 359 & 1246.204 \\ 28 & 29 & 1246.820 \\ 387 & 175 & 1247.586 \\ 102 & 241 & 1248.809 \\ & & \end{array}$

$\begin{array}{llll}81595.25 & 1225.5902 & 0.0032 & 1.91\end{array}$ $\begin{array}{lllll}81556.54 & 1226.1452 & 0.0021 & 0.13\end{array}$ $\begin{array}{llll}81527.35 & 1226.5828 & 0.0019 & 0.04\end{array}$ $\begin{array}{lllll}81514.95 & 1226.7685 & 0.0030 & -0.02\end{array}$ $\begin{array}{lllll}81486.18 & 1227.1971 & 0.0041 & -0.32\end{array}$ $\begin{array}{llll}81471.70 & 1227.4175 & 0.0030 & -0.17\end{array}$ $\begin{array}{lllll}81294.19 & 1230.0990 & 0.0020 & -0.08\end{array}$ $\begin{array}{lllll}81266.16 & 1230.5233 & 0.0030 & -0.08\end{array}$ $\begin{array}{llll}81240.42 & 1230.9119 & 0.0030 & -0.16\end{array}$ $\begin{array}{llll}81231.88 & 1231.0483 & 0.0030 & 0.30\end{array}$ $\begin{array}{llll}81225.78 & 1231.1355 & 0.0021 & -0.04\end{array}$ $\begin{array}{lllll}81193.95 & 1231.6211 & 0.0041 & 0.15\end{array}$ $\begin{array}{llll}81185.44 & 1231.7437 & 0.0022 & -0.28\end{array}$ $\begin{array}{lllll}81131.09 & 1232.5695 & 0.0019 & -0.24\end{array}$ $\begin{array}{llll}81112.91 & 1232.8506 & 0.0020 & 0.08\end{array}$ $\begin{array}{rrrrr}81103.18 & 1232.9961 & 0.0024 & -0.08\end{array}$ $\begin{array}{lllll}81048.44 & 1233.8328 & 0.0020 & 0.18\end{array}$ $\begin{array}{llll}81043.98 & 1233.8999 & 0.0041 & 0.12\end{array}$ $\begin{array}{lllll}81031.95 & 1234.0734 & 0.0030 & -0.51\end{array}$ $\begin{array}{lllll}81027.35 & 1234.1463 & 0.0040 & -0.32\end{array}$ $\begin{array}{llll}80999.84 & 1234.5722 & 0.0020 & 0.12\end{array}$ $\begin{array}{llll}80942.82 & 1235.4448 & 0.0021 & 0.31\end{array}$ $\begin{array}{lllll}80922.57 & 1235.7628 & 0.0024 & 0.89\end{array}$ $\begin{array}{lllll}80871.94 & 1236.5231 & 0.0030 & 0.02\end{array}$ $\begin{array}{lllll}80848.04 & 1236.8957 & 0.0019 & 0.48\end{array}$ $\begin{array}{lllll}80777.97 & 1237.9616 & 0.0023 & 0.02\end{array}$ $\begin{array}{lllll}80765.94 & 1238.1489 & 0.0019 & 0.21\end{array}$ $\begin{array}{lllll}80761.27 & 1238.2276 & 0.0030 & 0.67\end{array}$ $\begin{array}{lllll}80599.95 & 1240.6977 & 0.0020 & 0.14\end{array}$ $\begin{array}{lllll}80529.89 & 1241.7779 & 0.0018 & 0.19\end{array}$ $\begin{array}{llll}80504.84 & 1242.1620 & 0.0032 & 0.04\end{array}$ $\begin{array}{llll}80494.18 & 1242.3331 & 0.0030 & 0.47\end{array}$ $\begin{array}{lllll}80455.14 & 1242.9294 & 0.0030 & 0.05\end{array}$ $\begin{array}{llll}80449.85 & 1243.0108 & 0.0050 & 0.03\end{array}$ $\begin{array}{lllll}80406.05 & 1243.6900 & 0.0030 & 0.16\end{array}$ $\begin{array}{llll}80354.68 & 1244.4831 & 0.0031 & 0.03\end{array}$ $\begin{array}{lllll}80332.53 & 1244.8239 & 0.0040 & -0.12\end{array}$ $\begin{array}{lllll}80322.36 & 1244.9774 & 0.0024 & -0.38\end{array}$ $\begin{array}{lllll}80263.95 & 1245.8906 & 0.0021 & 0.08\end{array}$ $\begin{array}{lllll}80260.65 & 1245.9396 & 0.0040 & -0.05\end{array}$ $\begin{array}{llll}80243.66 & 1246.2064 & 0.0023 & 0.13\end{array}$ $\begin{array}{lllll}80204.04 & 1246.8262 & 0.0031 & 0.40\end{array}$ $\begin{array}{lllll}80154.76 & 1247.5875 & 0.0020 & 0.06\end{array}$ $\begin{array}{llll}80076.32 & 1248.8133 & 0.0034 & 0.30\end{array}$ $\begin{array}{lllll}-0.029 & 11 & 15 & 5 \mathrm{~d}^{5} 6 \mathrm{~s} & 75164.22(2)\end{array}$ - 11 - $15=5 d^{5} 6 s \quad 75164.22(2)$ 0.000 0.005 0.003 0.001

0.001

0.002

$-0.005$

0.001

$-0.002$

0.004

0.004

$-0.001$

0.001

$-0.003$

$-0.002$

0.008

0.005

$-0.005$

$-0.014$

0.000

$-0.007$

0.000

$-0.003$

$-0.010$

$-0.002$

$-0.003$

$-0.001$

$-0.007$

$-0.001$

0.000

$-0.002$

0.000

0.002

0.006

$-0.001$

$-0.002$

$-0.006$

$-0.001$ $-0.005$
$156757.56(3) \quad \mathrm{pb} * 100, \mathrm{~m}$

$154293.71(6) \quad \mathrm{pb}$

145439.95(4) $\mathrm{pb}$

137142.67(1) pb

$113462.35(4) \quad \mathrm{pb}$

$140678.86(1) \quad \mathrm{pb} \quad$ Ir III

140526.92(3) pb

140473.23(2) $\mathrm{pb}$

140473.23(2) $\quad$ pb $\quad$ Ir III (150)

$147341.74(3) \quad \mathrm{pb}$

154293.71(6) pb

141466.77(2) pb

$157481.05(5) \quad \mathrm{pb}$

135595.44(4) pb

$152304.45(5) \quad \mathrm{pb}$

$155160.61(5) \quad \mathrm{pb}$

162653.94(5) pb

148135.70(2) pb O IV 2d ord

157327.79(6) pb* O IV 2d ord

155085.02(3) $\mathrm{pb} *$

139739.81(5) pb

144855.15(3) $\mathrm{pb}$

153658.98(5) pb*

$157167.25(4) \quad \mathrm{pb}$

$140054.55(3) \mathrm{pb}$

133037.83(3) pb

139998.38(4) pb

$153828.48(4) \quad \mathrm{pb}$

$144512.45(5) \quad \mathrm{pb}$

139739.81(5) pb 700

$165239.60(5) \quad \mathrm{pb}$

150235.09(3) pb

$153192.39(7) \quad \mathrm{pb}$

$150748.58(1) \quad \mathrm{pb}$

140678.86(1) pb

139094.74(6) pb

161684.04(1) pb

135950.44(1) pb*

156757.56(3) pb

$159762.71(5) \quad \mathrm{pb}$

134707.64(2) pb

151395.26(3) pb*

149313.18(6) pb

146186.18(3) pb 


\begin{tabular}{|c|c|c|c|c|c|c|}
\hline 176 & 44 & 1249.857 & & 1249.8605 & & \\
\hline 2105 & 1616 & 1251.806 & & 1251.8053 & 0.0030 & -0.05 \\
\hline 281 & 735 & 1252.243 & 79856.72 & 1252.2406 & 0.0030 & -0.14 \\
\hline 65 & 345 & 1252.699 & 79827.64 & 1252.6886 & 0.0023 & -0.66 \\
\hline 587 & 911 & 1252.829 & 9819.34 & 1252.8312 & 0.0021 & 0.13 \\
\hline 21 & 29 & 1253.245 & & 253.2533 & & 0.55 \\
\hline 267 & 1290 & 254.327 & 79724.00 & .3310 & 0024 & 0.23 \\
\hline 66 & 34 & 1254.891 & & 966 & 0060 & 0.37 \\
\hline 419 & 1067 & 1255 & & & & 0.19 \\
\hline 458 & 118 & 1256 & 06 & 99 & & -0.43 \\
\hline 241 & 99 & & & & & -0.01 \\
\hline 304 & 839 & 90 & 45 & & & 0.25 \\
\hline 146 & 468 & & & & & \\
\hline 350 & 144 & 1258 & & & & 0.36 \\
\hline 165 & 37 & 1258.8 & & & & 0.10 \\
\hline 69 & 58 & & & & & 0.62 \\
\hline 263 & 266 & 125 & & & & -0.61 \\
\hline 595 & 749 & & & & & 0.02 \\
\hline 727 & 1074 & 125 & & & & -0.03 \\
\hline 231 & 160 & & & & & 0.13 \\
\hline 487 & 101 & 125 & & & & -0.11 \\
\hline 349 & 255 & 126 & & & & -0.19 \\
\hline 252 & 342 & & & & & -0.16 \\
\hline 268 & 630 & 126 & & & & 0.02 \\
\hline 735 & 1168 & 126 & & & & 0.53 \\
\hline 44 & 31 & & & & & 0.65 \\
\hline 80 & 71 & & & & & 0.02 \\
\hline 416 & 433 & & & & & 0.32 \\
\hline 97 & 199 & 126 & & & & -0.45 \\
\hline 58 & 72 & 126 & & & & 0.36 \\
\hline 113 & 37 & 126 & & & & 0.12 \\
\hline 134 & 291 & $126^{\circ}$ & & & & 0.18 \\
\hline 198 & 114 & 126 & & & & -0.15 \\
\hline 93 & 50 & & & & & 0.01 \\
\hline 137 & 99 & 1269 & & & & -0.27 \\
\hline 196 & 35 & & & & & 0.10 \\
\hline 331 & 359 & 1270.225 & 78726.23 & 1270.2252 & & 0.03 \\
\hline 113 & 195 & 1271.424 & & 127 & & -0.09 \\
\hline 227 & 219 & 1271 & & 127 & & 0.01 \\
\hline 298 & 731 & 1272.694 & & 6902 & 0.0020 & -0.21 \\
\hline 66 & 58 & & & & & 0.86 \\
\hline 563 & 97 & 1273.884 & & 1273.8841 & & 0.02 \\
\hline 1791 & 1091 & 1274.142 & & 1274.1345 & 0.0030 & -0.47 \\
\hline 112 & 286 & 1274.295 & 78474.74 & 1274.2787 & 0.0023 & -1.03 \\
\hline
\end{tabular}

$\begin{array}{lllll}-0.003 & 8 & 16 & 5 d^{5} 6 s & 84734.80(4)\end{array}$ $\begin{array}{lllll}0.001 & 5 & 22 & 5 d^{5} 6 s & 59210.11(5)\end{array}$

0.002

0.010

$-0.002$

$\begin{array}{lllll}-0.009 & 15 & 23 & 5 d^{5} 6 \mathrm{~s} & 55627.70(2)\end{array}$

$\begin{array}{lllll}-0.004 & 5 & 18 & 5 d^{5} 6 s & 58740.09(6)\end{array}$

$\begin{array}{lllll}-0.006 & 12 & 15 & 5 d^{5} 6 s & 75164.22(2)\end{array}$

$\begin{array}{lllll}-0.003 & 11 & 22 & 5 d^{5} 6 s & 58041.98(4)\end{array}$

$\begin{array}{lllll}0.007 & 7 & 13 & 5 d^{5} 6 s & 90123.22(5)\end{array}$

$\begin{array}{lllll}0.000 & 15 & 20 & 5 d^{5} 6 s & 72737.30(6)\end{array}$

$\begin{array}{lllll}-0.004 & 16 & 23 & 5 d^{5} 6 s & 54464.11(3)\end{array}$

$\begin{array}{lllll}-0.006 & 11 & 26 & 5 d^{5} 6 s & 71191.62(4)\end{array}$

$\begin{array}{lllll}-0.006 & 9 & 14 & 5 d^{5} 6 s & 82458.31(3)\end{array}$

$-0.002$

$-0.010$

0.010

0.000

0.000

$-0.002$

0.002

0.003

0.003

0.000

$-0.008$

$-0.010$

0.000

$-0.005$

0.007

$-0.006$

$-0.002$

$-0.003$

0.002

0.000

0.004

$-0.002$

0.000

0.001

0.000

0.003

$-0.014$

0.000

0.008
0.017

\section{$5 \quad 9 \mathrm{~d}^{5} 6 \mathrm{~s}-85249$}

$\begin{array}{llll}8 & 21 & 5 \mathrm{~d}^{5} 6 \mathrm{~s} & 59206.99(2)\end{array}$

$\begin{array}{llll}4 & 9 & 5 d^{5} 6 s & 95628.69(5)\end{array}$

$\begin{array}{llll}10 & 18 & 5 d^{5} 6 s & 58740.09(6)\end{array}$

$\begin{array}{lllll}6 & 14 & 5 d^{5} 6 \mathrm{~s} & 91240.96(6)\end{array}$

$\begin{array}{llll}8 & 13 & 5 \mathrm{~d}^{5} 6 \mathrm{~s} & 90123.22(5)\end{array}$

$\begin{array}{llll}14 & 21 & 5 \mathrm{~d}^{5} 6 \mathrm{~s} & 74057.35(4)\end{array}$

$\begin{array}{llll}16 & 25 & 5 d^{5} 6 s & 66110.16(3)\end{array}$

$\begin{array}{llll}7 & 17 & 5 d^{5} 6 s & 87782.64(4)\end{array}$

$\begin{array}{llll}10 & 21 & 5 d^{5} 6 s & 59206.99(2)\end{array}$

$\begin{array}{llll}16 & 20 & 5 d^{5} 6 s & 63912.64(4)\end{array}$

$\begin{array}{llll}7 & 5 & 5 d^{5} 6 s & 65108.69(0)\end{array}$

$\begin{array}{llll}7 & 12 & 5 d^{5} 6 s & 84005.15(4)\end{array}$

$\begin{array}{llll}10 & 20 & 5 d^{5} 6 s & 76493.69(3)\end{array}$

$\begin{array}{llll}13 & 23 & 5 \mathrm{~d}^{5} 6 \mathrm{~s} & 55627.70(2)\end{array}$

$\begin{array}{llll}8 & 13 & 5 d^{5} 6 s & 90497.75(4)\end{array}$

$\begin{array}{llll}10 & 22 & 5 d^{5} 6 s & 59210.11(5)\end{array}$

$\begin{array}{llll}14 & 19 & 5 d^{5} 6 s & 74545.24(3)\end{array}$

$\begin{array}{llll}4 & 18 & 5 d^{5} 6 s & 80577.63(6)\end{array}$

$\begin{array}{llll}7 & 11 & 5 d^{5} 6 s & 75494.15(7)\end{array}$

$\begin{array}{llll}8 & 9 & 5 d^{5} 6 s & 85249.67(3)\end{array}$

$\begin{array}{llll}11 & 23 & 5 d^{5} 6 s & 69158.48(5)\end{array}$

$\begin{array}{llll}10 & 14 & 5 \mathrm{~d}^{5} 6 \mathrm{~s} & 50417.92(2)\end{array}$

$\begin{array}{llll}9 & 20 & 5 d^{5} 6 s & 72737.30(6)\end{array}$

$\begin{array}{llll}14 & 23 & 5 d^{5} 6 s & 54464.11(3)\end{array}$

$\begin{array}{llll}9 & 25 & 5 d^{5} 6 s & 66110.16(3)\end{array}$

$\begin{array}{llll}5 & 17 & 5 d^{5} 6 s & 87782.64(4)\end{array}$

$\begin{array}{llll}5 & 18 & 5 d^{5} 6 \mathrm{~s} & 58740.09(6)\end{array}$ $\begin{array}{llll}7 & 25 & 5 \mathrm{~d}^{5} 6 \mathrm{~s} & 85249.67(3) \\ & 25 & & 6110.16(3)\end{array}$
164743.73(4) pb

139094.74(6) pb

135484.56(1) pb

132088.18(4) pb

143731.85(5) pb

135420.03(3) pb*

$138463.86(7) \quad \mathrm{pb}$

$154852.06(2) \quad \mathrm{pb} *$

137685.88(5) pb

169715.71(6) pb

152304.45(5) pb

$134025.31(4) \quad \mathrm{pb}$

$150706.05(4) \quad \mathrm{pb} \quad \mathrm{bl}(\mathrm{Al} \mathrm{IV})$

61902.01(4) pb

164689.65(3) pb

145536.69(3) pb

$138612.56(2) \quad \mathrm{pb}$

175027.06(4) pb O V 2d ord

138121.21(6) pb

170618.53(5) pb

169490.49(5) pb

$153412.13(4) \quad \mathrm{pb}$

145439.95(4) pb

167107.33(4) pb

138529.81(3) $\mathrm{pb}$

143156.95(4) pb*

$144347.40(1) \mathrm{pb}$

$163240.75(5) \quad \mathrm{pb}$

$155597.91(4) \quad \mathrm{pb}$

134707.64(2) pb

169490.49(5) pb

138121.21(6) pb

$153412.13(4) \quad \mathrm{pb}$

159416.27(6) pb

154293.71(6) pb

163997.14(4) pb

147884.68(4) pb

129069.98(3) pb

$151364.78(6) \quad \mathrm{pb}$

133037.83(3) pb

$144626.15(2) \quad \mathrm{pc} *$

$166282.72(5) \quad \mathrm{pc}$

137224.74(7) pc

$137685.88(5) \quad$ pc $*$ 


$\begin{array}{rrr}777 & 286 & 1274.295 \\ 456 & 158 & 1274.359 \\ 194 & 354 & 1274.401 \\ 875 & 1131 & 1275.711 \\ 200 & 208 & 1276.495 \\ 1663 & 959 & 1277.186 \\ 124 & 188 & 1277.676 \\ 433 & 499 & 1277.998 \\ 412 & 181 & 1279.475 \\ 296 & 549 & 1280.947 \\ 209 & 191 & 1281.064 \\ 120 & 106 & 1281.312 \\ 251 & 114 & 1281.572 \\ 233 & 137 & 1281.687 \\ 256 & 91 & 1282.396 \\ 445 & 167 & 1282.727 \\ 629 & 697 & 1283.169 \\ 460 & 109 & 1283.379 \\ 58 & 44 & 1284.732 \\ 67 & 49 & 1284.881 \\ 1761 & 1035 & 1285.172 \\ 448 & 528 & 1286.226 \\ 1552 & 1048 & 1287.029 \\ 143 & 164 & 1287.554 \\ 415 & 987 & 1288.262 \\ 175 & 396 & 1289.052 \\ 386 & 730 & 1289.431 \\ 168 & 90 & 1289.499 \\ 429 & 228 & 1289.767 \\ 83 & 28 & 1291.031 \\ 89 & 108 & 1291.821 \\ 13 & 22 & 1292.360 \\ 140 & 74 & 1292.595 \\ 195 & 365 & 1293.072 \\ 173 & 97 & 1293.973 \\ 85 & 90 & 1294.403 \\ 160 & 376 & 1294.861 \\ 257 & 505 & 1295.016 \\ 160 & 47 & 1295.821 \\ 3306 & 1627 & 1297.657 \\ 212 & 86 & 1297.980 \\ 111 & 73 & 1299.371 \\ 139 & 98 & 1299.439 \\ 2148 & 1224 & 1299.964\end{array}$

$\begin{array}{lllll}78474.74 & 1274.2952 & 0.0030 & -0.01\end{array}$ $\begin{array}{lllll}78470.84 & 1274.3592 & 0.0040 & 0.03\end{array}$ $\begin{array}{llll}78468.23 & 1274.4050 & 0.0020 & 0.23\end{array}$ $\begin{array}{lllll}78387.67 & 1275.7167 & 0.0023 & 0.36\end{array}$ $\begin{array}{lllll}78339.49 & 1276.4938 & 0.0031 & -0.10\end{array}$ $\begin{array}{llll}78297.13 & 1277.1898 & 0.0031 & 0.23\end{array}$ $\begin{array}{lllll}78267.12 & 1277.6744 & 0.0040 & -0.08\end{array}$ $\begin{array}{llll}78247.38 & 1278.0026 & 0.0022 & 0.28\end{array}$ $\begin{array}{lllll}78157.06 & 1279.4754 & 0.0040 & 0.03\end{array}$ $\begin{array}{lllll}78067.27 & 1280.9469 & 0.0020 & 0.02\end{array}$ $\begin{array}{lllll}78060.12 & 1281.0655 & 0.0030 & 0.10\end{array}$ $\begin{array}{lllll}78044.98 & 1281.3168 & 0.0024 & 0.27\end{array}$ $\begin{array}{llll}78029.14 & 1281.5763 & 0.0040 & 0.23\end{array}$ $\begin{array}{lllll}78022.17 & 1281.6924 & 0.0030 & 0.33\end{array}$ $\begin{array}{llll}77979.02 & 1282.3957 & 0.0031 & -0.03\end{array}$ $\begin{array}{rrrrr}77958.93 & 1282.7298 & 0.0041 & 0.19\end{array}$ $\begin{array}{lllll}77932.05 & 1283.1657 & 0.0031 & -0.21\end{array}$ $\begin{array}{lllll}77919.27 & 1283.3817 & 0.0030 & 0.13\end{array}$ $\begin{array}{lllll}77837.27 & 1284.7370 & 0.0032 & 0.33\end{array}$ $\begin{array}{llll}77828.20 & 1284.8782 & 0.0040 & -0.19\end{array}$ $\begin{array}{llll}77810.60 & 1285.1721 & 0.0030 & 0.01\end{array}$ $\begin{array}{llll}77746.85 & 1286.2382 & 0.0030 & 0.75\end{array}$ $\begin{array}{lllll}77698.33 & 1287.0304 & 0.0040 & 0.09\end{array}$ $\begin{array}{lllll}77666.66 & 1287.5771 & 0.0041 & 1.41\end{array}$ $\begin{array}{llll}77623.97 & 1288.2602 & 0.0020 & -0.10\end{array}$ $\begin{array}{lllll}77576.41 & 1289.0518 & 0.0030 & 0.01\end{array}$ $\begin{array}{lllll}77553.61 & 1289.4331 & 0.0021 & 0.15\end{array}$ $\begin{array}{lllll}77549.47 & 1289.5064 & 0.0030 & 0.42\end{array}$ $\begin{array}{llll}77533.35 & 1289.7709 & 0.0032 & 0.20\end{array}$ $\begin{array}{lllll}77457.47 & 1291.0396 & 0.0031 & 0.51\end{array}$ $\begin{array}{lllll}77410.11 & 1291.8206 & 0.0020 & -0.02\end{array}$ $\begin{array}{llll}77377.83 & 1292.3562 & 0.0024 & -0.22\end{array}$ $\begin{array}{lllll}77363.75 & 1292.5967 & 0.0030 & 0.10\end{array}$ $\begin{array}{lllll}77335.23 & 1293.1025 & 0.0050 & 1.84\end{array}$ $\begin{array}{llll}77281.39 & 1293.9731 & 0.0030 & 0.03\end{array}$ $\begin{array}{llll}77255.68 & 1294.4052 & 0.0030 & 0.12\end{array}$ $\begin{array}{lllll}77228.35 & 1294.8584 & 0.0023 & -0.17\end{array}$ $\begin{array}{lllll}77219.14 & 1295.0150 & 0.0023 & -0.04\end{array}$ $\begin{array}{lllll}77171.18 & 1295.8217 & 0.0060 & 0.06\end{array}$ $\begin{array}{rrrrr}77061.96 & 1297.6571 & 0.0070 & 0.00\end{array}$ $\begin{array}{lllll}77042.80 & 1297.9768 & 0.0030 & -0.18\end{array}$ $\begin{array}{lllll}76960.34 & 1299.3579 & 0.0040 & -0.75\end{array}$ $\begin{array}{llll}76956.30 & 1299.4423 & 0.0030 & 0.21\end{array}$ $\begin{array}{llll}76925.20 & 1299.9743 & 0.0040 & 0.60\end{array}$ $\begin{array}{lllll}0.000 & 7 & 14 & 5 d^{5} 6 s & 91240.96(6)\end{array}$ $\begin{array}{lllll}8 & 16 & 5 \mathrm{~d}^{5} 6 \mathrm{~s} & 84734.80(4)\end{array}$ $\begin{array}{lllll}-0.004 & 12 & 24 & 5 d^{5} 6 s & 59232.65(3)\end{array}$ $\begin{array}{lllll}-0.006 & 12 & 22 & 5 d^{5} 6 s & 58041.98(4)\end{array}$

$\begin{array}{llllll}0.002 & 8 & 9 & 5 \mathrm{~d}^{5} 6 \mathrm{~s} & 60272.97(1)\end{array}$

$\begin{array}{lllll}-0.004 & 9 & 19 & 5 d^{5} 6 s & 73067.88(5)\end{array}$

$\begin{array}{llllll}0.001 & 9 & 6 & 5 d^{5} 6 \mathrm{~s} & 67091.85(1)\end{array}$

$-0.005$

$-0.001$

0.000

$-0.002$

$-0.004$

$-0.004$

$-0.005$

0.000

$-0.003$

0.003

$-0.002$

$-0.005$

0.003

0.000

$-0.012$

$-0.001$

$-0.023$

0.002

0.000

$-0.002$

$-0.007$

$-0.003$

$-0.009$

0.000

0.004

$-0.002$

$-0.031$

0.000

$-0.002$

0.003

0.001

$-0.001$

0.000

0.003

0.013

$-0.004$

$-0.010$
169715.71(6) pc

63205.61(3) pc

137700.64(3) pc

$136429.29(5) \quad \mathrm{pc}$

138612.56(2) pc

$151364.78(6)$ pc

$145359.05(2) \quad \mathrm{pc}$

$152304.45(5) \quad \mathrm{pc}$

159762.71(5) pc

137277.36(4) pc

$148358.78(3) \quad \mathrm{pc}$

137277.36(4) pc

$153193.13(2) \quad \mathrm{pc}$

$147763.22(4) \quad \mathrm{pc}$

$157481.05(5) \quad \mathrm{pc}$

$157167.25(4) \quad$ pc

$151000.14(5) \quad \mathrm{pc}$

162653.94(5) pc

148135.70(2) pc

152373.63(3) pc

159416.27(6) pc

148937.72(5) pc

153192.39(7) pc

157167.25(4) pc* bl(Ir V)

132088.18(4) pc

$133204.10(2) \quad \mathrm{pc}$

135595.44(4) pc

$156757.56(3) \quad \mathrm{pc}$

153828.48(4) pc

$165239.60(5) \quad \mathrm{pc}$

133037.83(3) pc

$135420.03(3) \quad \mathrm{pc} *$

$153658.98(5) \quad \mathrm{pc}$

$159791.70(4) \quad \mathrm{pc} * 100, \mathrm{bl}$

145536.69(3) pc

156757.56(3) pc

141141.16(4) pc

136429.29(5) pc

$159629.42(3) \quad$ pc

$152556.11(8) \quad \mathrm{pc}$

147341.74(3) pc

164743.73(4) pc

137229.06(2) pc

149661.90(7) pc Ir III 


$\begin{array}{rrr}283 & 107 & 1300.070 \\ 137 & 60 & 1300.334 \\ 21 & 57 & 1302.859 \\ 1000 & 555 & 1302.931 \\ 13 & 25 & 1303.029 \\ 179 & 130 & 1303.248 \\ 22 & 69 & 1304.842 \\ 140 & 38 & 1305.313 \\ 10 & 16 & 1305.476 \\ 988 & 912 & 1305.899 \\ 14 & 30 & 1308.708 \\ 290 & 90 & 1309.080 \\ 23 & 59 & 1309.143 \\ 331 & 132 & 1309.272 \\ 415 & 354 & 1309.474 \\ 136 & 122 & 1309.537 \\ 46 & 35 & 1310.939 \\ 24 & 26 & 1310.997 \\ 2631 & 976 & 1311.351 \\ 19 & 39 & 1311.768 \\ 47 & 50 & 1312.125 \\ 182 & 550 & 1312.330 \\ 128 & 172 & 1312.552 \\ 148 & 55 & 1313.038 \\ 115 & 18 & 1313.725 \\ 490 & 733 & 1314.257 \\ 416 & 264 & 1315.633 \\ 224 & 34 & 1316.003 \\ 24 & 21 & 1316.085 \\ 134 & 58 & 1317.929 \\ 196 & 145 & 1318.048 \\ 1447 & 695 & 1319.260 \\ 290 & 165 & 1319.495 \\ 925 & 767 & 1320.001 \\ 200 & 54 & 1320.481 \\ 208 & 76 & 1320.560 \\ 273 & 118 & 1320.614 \\ 142 & 120 & 1321.034 \\ 82 & 22 & 1321.736 \\ 72 & 23 & 1322.437 \\ 44 & 50 & 1324.489 \\ 30 & 40 & 1324.945 \\ 241 & 63 & 1324.974 \\ 622 & 278 & 1325.232\end{array}$

$\begin{array}{lllll}76918.93 & 1300.0784 & 0.0030 & 0.49\end{array}$ $\begin{array}{lllll}76903.34 & 1300.3323 & 0.0030 & -0.08\end{array}$ $\begin{array}{lllll}76754.29 & 1302.8477 & 0.0033 & -0.65\end{array}$ $\begin{array}{lllll}76750.03 & 1302.9289 & 0.0030 & -0.13\end{array}$ $\begin{array}{lllll}76744.28 & 1303.0428 & 0.0030 & 0.83\end{array}$ $\begin{array}{lllll}76731.38 & 1303.2461 & 0.0051 & -0.10\end{array}$ $\begin{array}{lllll}76637.62 & 1304.8447 & 0.0050 & 0.15\end{array}$ $\begin{array}{lllll}76609.97 & 1305.3198 & 0.0031 & 0.39\end{array}$ $\begin{array}{llll}76600.39 & 1305.4861 & 0.0020 & 0.57\end{array}$ $\begin{array}{llll}76575.61 & 1305.8942 & 0.0023 & -0.27\end{array}$ $\begin{array}{llll}76411.25 & 1308.7099 & 0.0024 & 0.12\end{array}$ $\begin{array}{lllll}76389.52 & 1309.0822 & 0.0030 & 0.12\end{array}$ $\begin{array}{llll}76385.87 & 1309.1519 & 0.0022 & 0.54\end{array}$ $\begin{array}{lllll}76378.31 & 1309.2676 & 0.0060 & -0.27\end{array}$ $\begin{array}{llll}76366.52 & 1309.4701 & 0.0022 & -0.25\end{array}$ $\begin{array}{lllll}76362.85 & 1309.5383 & 0.0021 & 0.06\end{array}$ $\begin{array}{llll}76281.19 & 1310.9344 & 0.0022 & -0.28\end{array}$ $\begin{array}{lllll}76277.85 & 1311.0014 & 0.0031 & 0.28\end{array}$ $\begin{array}{lllll}76257.23 & 1311.3524 & 0.0050 & 0.08\end{array}$ $\begin{array}{llll}76232.98 & 1311.7622 & 0.0024 & -0.36\end{array}$ $\begin{array}{lllll}76212.24 & 1312.1114 & 0.0023 & -0.80\end{array}$ $\begin{array}{lllll}76200.35 & 1312.3287 & 0.0030 & -0.07\end{array}$ $\begin{array}{lllll}76187.43 & 1312.5533 & 0.0023 & 0.05\end{array}$ $\begin{array}{lllll}76159.24 & 1313.0338 & 0.0060 & -0.26\end{array}$ $\begin{array}{lllll}76119.46 & 1313.7321 & 0.0100 & 0.44\end{array}$ $\begin{array}{lllll}76088.64 & 1314.2570 & 0.0021 & 0.02\end{array}$ $\begin{array}{lllll}76009.03 & 1315.6316 & 0.0023 & -0.09\end{array}$ $\begin{array}{lllll}75987.66 & 1315.9999 & 0.0040 & -0.19\end{array}$ $\begin{array}{lllll}75982.92 & 1316.0781 & 0.0021 & -0.41\end{array}$ $\begin{array}{lllll}75876.65 & 1317.9309 & 0.0040 & 0.14\end{array}$ $\begin{array}{lllll}75869.78 & 1318.0469 & 0.0030 & -0.06\end{array}$ $\begin{array}{llll}75800.10 & 1319.2610 & 0.0051 & 0.09\end{array}$ $\begin{array}{lllll}75786.57 & 1319.4953 & 0.0050 & 0.02\end{array}$ $\begin{array}{llll}75757.49 & 1320.0003 & 0.0040 & -0.07\end{array}$ $\begin{array}{lllll}75729.99 & 1320.4803 & 0.0030 & -0.03\end{array}$ $\begin{array}{lllll}75725.47 & 1320.5555 & 0.0060 & -0.24\end{array}$ $\begin{array}{rrrrr}75722.38 & 1320.6182 & 0.0030 & 0.27\end{array}$ $\begin{array}{lllll}75698.27 & 1321.0289 & 0.0022 & -0.30\end{array}$ $\begin{array}{lllll}75658.07 & 1321.7266 & 0.0031 & -0.54\end{array}$ $\begin{array}{lllll}75617.99 & 1322.4422 & 0.0030 & 0.32\end{array}$ $\begin{array}{llll}75500.79 & 1324.4919 & 0.0030 & 0.14\end{array}$ $\begin{array}{rrrrr}75474.82 & 1324.9422 & 0.0031 & -0.17\end{array}$ $\begin{array}{lllll}75473.18 & 1324.9752 & 0.0040 & 0.07\end{array}$ $\begin{array}{llll}75458.47 & 1325.2386 & 0.0040 & 0.36\end{array}$ $\begin{array}{lllll}-0.008 & 14 & 20 & 5 \mathrm{~d}^{5} 6 \mathrm{~s} & 76493.69(3)\end{array}$ $\begin{array}{llllll}0.001 & 12 & 18 & 5 \mathrm{~d}^{5} 6 \mathrm{~s} & 80577.63(6)\end{array}$

$\begin{array}{lllll}0.011 & 13 & 25 & 5 \mathrm{~d}^{5} 6 \mathrm{~s} & 66110.16(3)\end{array}$

$\begin{array}{lllll}0.002 & 11 & 18 & 5 d^{5} 6 \mathrm{~s} & 80577.63(6)\end{array}$

$\begin{array}{llllll}-0.014 & 10 & 21 & 5 \mathrm{~d}^{5} 6 \mathrm{~s} & 59206.99(2)\end{array}$

$\begin{array}{lllll}0.002 & 2 & 14 & 5 \mathrm{~d}^{5} 6 \mathrm{~s} & 82458.31(3)\end{array}$

$\begin{array}{lllll}-0.003 & 13 & 7 & 5 d^{6} & 46564.59(2)\end{array}$

$\begin{array}{lllll}-0.007 & 7 & 13 & 5 d^{5} 6 s & 90497.75(4)\end{array}$

\begin{tabular}{lllll}
-0.010 & 15 & 19 & $5 \mathrm{~d}^{5} 6 \mathrm{~s}$ & $68255.33(2)$ \\
\hline
\end{tabular}

$\begin{array}{lllll}0.005 & 9 & 20 & 5 \mathrm{~d}^{5} 6 \mathrm{~s} & 72737.30(6)\end{array}$

$\begin{array}{llllll}-0.002 & 10 & 23 & 5 d^{5} 6 s & 54464.11(3)\end{array}$

$\begin{array}{lllll}-0.002 & 10 & 19 & 5 \mathrm{~d}^{5} 6 \mathrm{~s} & 79208.51(3)\end{array}$

$\begin{array}{lllll}-0.009 & 17 & 22 & 5 \mathrm{~d}^{5} 6 \mathrm{~s} & 59210.11(5)\end{array}$

$\begin{array}{lllll}0.005 & 3 & 13 & 5 \mathrm{~d}^{5} 6 \mathrm{~s} & 90497.75(4)\end{array}$

$\begin{array}{lllll}0.004 & 17 & 23 & 5 \mathrm{~d}^{5} 6 \mathrm{~s} & 69158.48(5)\end{array}$

$\begin{array}{llllll}-0.001 & 17 & 24 & 5 \mathrm{~d}^{5} 6 \mathrm{~s} & 59232.65(3)\end{array}$

$\begin{array}{lllll}0.005 & 16 & 23 & 5 \mathrm{~d}^{5} 6 \mathrm{~s} & 69158.48(5)\end{array}$

$\begin{array}{llllll}-0.005 & 6 & 21 & 5 d^{5} 6 s & 59206.99(2)\end{array}$

$\begin{array}{lllll}-0.001 & 3 & 14 & 5 d^{5} 6 s & 91240.96(6)\end{array}$

$\begin{array}{lllll}0.006 & 14 & 16 & 5 \mathrm{~d}^{6} & 52259.88(3)\end{array}$

$\begin{array}{lllll}0.014 & 15 & 21 & 5 \mathrm{~d}^{5} 6 \mathrm{~s} & 59206.99(2)\end{array}$

$\begin{array}{lllll}0.001 & 12 & 20 & 5 \mathrm{~d}^{5} 6 \mathrm{~s} & 72737.30(6)\end{array}$

$\begin{array}{llllll}-0.001 & 15 & 24 & 5 d^{5} 6 \mathrm{~s} & 59232.65(3)\end{array}$

$\begin{array}{lllll}0.004 & 5 & 13 & 5 \mathrm{~d}^{5} 6 \mathrm{~s} & 90123.22(5)\end{array}$

$\begin{array}{lllll}-0.008 & 6 & 9 & 5 \mathrm{~d}^{5} 6 \mathrm{~s} & 85249.67(3)\end{array}$

$\begin{array}{lllll}0.000 & 13 & 25 & 5 d^{5} 6 \mathrm{~s} & 66110.16(3)\end{array}$

$\begin{array}{lllll}0.002 & 15 & 22 & 5 \mathrm{~d}^{5} 6 \mathrm{~s} & 76295.33(5)\end{array}$

$\begin{array}{llllll}0.003 & 8 & 10 & 5 d^{5} 6 \mathrm{~s} & 88009.29(3) \\ 0.007 & 16 & 22 & 5 d^{5} 6 \mathrm{~s} & 5804.98(4)\end{array}$

$\begin{array}{lllll}0.007 & 16 & 22 & 5 \mathrm{~d}^{5} 6 \mathrm{~s} & 58041.98(4)\end{array}$

$\begin{array}{llllll}-0.002 & 13 & 19 & 5 d^{5} 6 s & 79208.51(3)\end{array}$

$\begin{array}{llllll}0.001 & 12 & 19 & 5 d^{5} 6 s & 73067.88(5)\end{array}$

$\begin{array}{lllll}-0.002 & 4 & 13 & 5 \mathrm{~d}^{5} 6 \mathrm{~s} & 90123.22(5)\end{array}$

$\begin{array}{lllll}0.000 & 8 & 12 & 5 d^{5} 6 \mathrm{~s} & 84005.15(4)\end{array}$

$\begin{array}{lllll}0.001 & 8 & 12 & 5 d^{5} 6 s & 84005.15(4)\end{array}$

$\begin{array}{llllll}0.001 & 12 & 14 & 5 \mathrm{~d}^{5} 6 \mathrm{~s} & 82458.31(3) \\ 0.004 & 8 & 10 & 5 \mathrm{~d}^{5} 6 \mathrm{~s} & 88009.29(3)\end{array}$

$\begin{array}{lllll}0.004 & 8 & 10 & 5 \mathrm{~d}^{5} 6 \mathrm{~s} & 88009.29(3)\end{array}$

$\begin{array}{lllll}-0.005 & 11 & 20 & 5 \mathrm{~d}^{5} 6 \mathrm{~s} & 81605.68(5)\end{array}$

$\begin{array}{lllll}0.005 & 16 & 21 & 5 \mathrm{~d}^{5} 6 \mathrm{~s} & 69741.38(3)\end{array}$

$\begin{array}{lllll}0.009 & 12 & 19 & 5 \mathrm{~d}^{5} 6 \mathrm{~s} & 79502.00(4)\end{array}$

$\begin{array}{lllll}-0.006 & 9 & 21 & 5 d^{5} 6 \mathrm{~s} & 69741.38(3)\end{array}$

$\begin{array}{lllll}-0.003 & 13 & 21 & 5 d^{5} 6 s & 59206.99(2)\end{array}$

$\begin{array}{llllll}0.003 & 13 & 24 & 5 d^{5} 6 \mathrm{~s} & 59232.65(3)\end{array}$

$\begin{array}{lllll}-0.001 & 7 & 16 & 5 d^{5} 6 \mathrm{~s} & 84734.80(4)\end{array}$

$-0.006$
$153412.13(4) \quad \mathrm{pc}$

157481.05(5) pc

$142865.10(2) \quad \mathrm{pc} *$

$157327.79(6) \quad \mathrm{pc}$

$135950.44(1) \quad \mathrm{pc} *$ $159189.79(3) \quad \mathrm{pc}$ $123202.06(3) \quad \mathrm{pc}$ 167107.33(4) pc $144855.15(3) \quad \mathrm{pc} *$ 149313.18(6) pc 130875.24(2) pc * 155597.91(4) pc 135595.44(4) pc $166876.33(4) \quad \mathrm{pc}$ $145525.25(5) \quad \mathrm{pc}$ $135595.44(4) \quad \mathrm{pc}$ 145439.95(4) pc 135484.56(1) pc $167498.11(7) \quad \mathrm{pc}$ 128493.21(4) pc $135420.03(3) \quad \mathrm{pc}$ $148937.72(5) \quad$ pc $\quad$ Ir III (400) $135420.03(3) \quad \mathrm{pc}$ 166282.72(5) pc * 161368.69(3) pc* $142198.78(4) \quad \mathrm{pc}$ $152304.45(5) \quad$ pc 163997.14(4) pc 134025.31(4) pc* $155085.02(3) \quad \mathrm{pc}$ 148937.72(5) pc 165923.23(6) pc $159791.70(4) \quad \mathrm{pc}$ 159762.71(5) pc* $158188.33(4) \quad \mathrm{pc}$ 163735.00(3) pc $157327.79(6) \quad \mathrm{pc}$ $145439.95(4) \quad \mathrm{pc}$ 155160.61(5) pc* 145359.05(2) pc* 134707.64(2) pc $134707.64(2) \quad \mathrm{pc}$ 160207.91(4) pc $163240.75(5) \quad \mathrm{pc}$ 


$\begin{array}{rrr}272 & 431 & 1327.070 \\ 21 & 137 & 1328.416 \\ 60 & 56 & 1328.683 \\ 126 & 399 & 1330.293 \\ 67 & 57 & 1331.486 \\ 123 & 85 & 1331.940 \\ 334 & 364 & 1332.094 \\ 21 & 35 & 1332.279 \\ 29 & 77 & 1332.833 \\ 159 & 302 & 1333.410 \\ 129 & 302 & 1333.410 \\ 899 & 239 & 1333.522 \\ 505 & 203 & 1334.078 \\ 130 & 125 & 1334.334 \\ 31 & 31 & 1335.086 \\ 522 & 272 & 1335.468 \\ 53 & 67 & 1336.620 \\ 105 & 106 & 1337.028 \\ 736 & 494 & 1337.933 \\ 102 & 21 & 1338.533 \\ 52 & 82 & 1338.617 \\ 648 & 238 & 1339.006 \\ 76 & 243 & 1339.181 \\ 28 & 32 & 1340.321 \\ 62 & 61 & 1340.372 \\ 291 & 88 & 1340.786 \\ 178 & 124 & 1340.962 \\ 46 & 24 & 1341.270 \\ 123 & 82 & 1341.535 \\ 20 & 36 & 1343.476 \\ 35 & 46 & 1343.796 \\ 131 & 53 & 1344.542 \\ 9 & 91 & 1344.644 \\ 326 & 61 & 1344.726 \\ 111 & 129 & 1345.282 \\ 85 & 149 & 1348.017 \\ 269 & 149 & 1348.500 \\ 106 & 89 & 1348.809 \\ 166 & 121 & 1349.188 \\ 68 & 303 & 1349.269 \\ 848 & 305 & 1349.769 \\ 57 & 88 & 1351.397 \\ 68 & 124 & 1351.878 \\ 99 & 48 & 1352.989\end{array}$

$\begin{array}{lllll}75353.97 & 1327.0701 & 0.0021 & 0.00\end{array}$ $\begin{array}{lllll}75277.61 & 1328.4238 & 0.0020 & 0.43\end{array}$ $\begin{array}{lllll}75262.51 & 1328.6847 & 0.0020 & 0.11\end{array}$ $\begin{array}{lllll}75171.43 & 1330.2889 & 0.0024 & -0.21\end{array}$ $\begin{array}{lllll}75104.04 & 1331.4835 & 0.0030 & -0.16\end{array}$ $\begin{array}{lllll}75078.43 & 1331.9511 & 0.0040 & 0.60\end{array}$ $\begin{array}{lllll}75069.79 & 1332.0998 & 0.0031 & 0.34\end{array}$ $\begin{array}{lllll}75059.36 & 1332.2624 & 0.0031 & -0.93\end{array}$ $\begin{array}{llll}75028.17 & 1332.8373 & 0.0050 & 0.26\end{array}$ $\begin{array}{llll}74995.69 & 1333.4071 & 0.0023 & -0.16\end{array}$ $\begin{array}{lllll}74995.69 & 1333.4301 & 0.0031 & 1.13\end{array}$ $\begin{array}{lllll}74989.41 & 1333.5140 & 0.0041 & -0.43\end{array}$ $\begin{array}{llll}74958.12 & 1334.0761 & 0.0040 & -0.12\end{array}$ $\begin{array}{lllll}74943.74 & 1334.3289 & 0.0032 & -0.30\end{array}$ $\begin{array}{llll}74901.52 & 1335.0855 & 0.0030 & -0.05\end{array}$ $\begin{array}{lllll}74880.10 & 1335.4635 & 0.0030 & -0.27\end{array}$ $\begin{array}{lllll}74815.58 & 1336.6268 & 0.0021 & 0.38\end{array}$ $\begin{array}{lllll}74792.74 & 1337.0296 & 0.0020 & 0.08\end{array}$ $\begin{array}{lllll}74742.16 & 1337.9385 & 0.0040 & 0.31\end{array}$ $\begin{array}{lllll}74708.66 & 1338.5279 & 0.0041 & -0.28\end{array}$ $\begin{array}{lllll}74703.98 & 1338.6019 & 0.0030 & -0.83\end{array}$ $\begin{array}{lllll}74682.24 & 1339.0059 & 0.0050 & -0.03\end{array}$ $\begin{array}{lllll}74672.52 & 1339.1777 & 0.0024 & -0.17\end{array}$ $\begin{array}{lllll}74608.99 & 1340.3070 & 0.0032 & -0.78\end{array}$ $\begin{array}{llll}74606.16 & 1340.3771 & 0.0022 & 0.29\end{array}$ $\begin{array}{llll}74583.10 & 1340.7885 & 0.0030 & 0.12\end{array}$ $\begin{array}{llll}74573.30 & 1340.9613 & 0.0023 & -0.07\end{array}$ $\begin{array}{lllll}74556.23 & 1341.2667 & 0.0030 & -0.16\end{array}$ $\begin{array}{lllll}74541.47 & 1341.5250 & 0.0060 & -0.56\end{array}$ $\begin{array}{lllll}74433.76 & 1343.4600 & 0.0030 & -0.91\end{array}$ $\begin{array}{lllll}74416.07 & 1343.7833 & 0.0024 & -0.69\end{array}$ $\begin{array}{lllll}74374.77 & 1344.5392 & 0.0051 & -0.15\end{array}$ $\begin{array}{lllll}74369.12 & 1344.6573 & 0.0040 & 0.73\end{array}$ $\begin{array}{llll}74364.59 & 1344.7159 & 0.0040 & -0.56\end{array}$ $\begin{array}{llll}74333.83 & 1345.2861 & 0.0024 & 0.20\end{array}$ $\begin{array}{lllll}74183.02 & 1348.0145 & 0.0030 & -0.16\end{array}$ $\begin{array}{lllll}74156.48 & 1348.4907 & 0.0031 & -0.50\end{array}$ $\begin{array}{lllll}74139.49 & 1348.8005 & 0.0050 & -0.46\end{array}$ $\begin{array}{lllll}74118.68 & 1349.1750 & 0.0040 & -0.69\end{array}$ $\begin{array}{llll}74114.19 & 1349.2802 & 0.0020 & 0.60\end{array}$ $\begin{array}{llll}74086.72 & 1349.7641 & 0.0040 & -0.30\end{array}$ $\begin{array}{lllll}73997.48 & 1351.4041 & 0.0030 & 0.37\end{array}$ $\begin{array}{lllll}73971.18 & 1351.8828 & 0.0040 & 0.26\end{array}$ $\begin{array}{lllll}73910.45 & 1352.9945 & 0.0030 & 0.32\end{array}$ $\begin{array}{lllllll}0.000 & 14 & 23 & 5 \mathrm{~d}^{5} 6 \mathrm{~s} & 69158.48(5) & 144512.45(5) & \mathrm{pc}\end{array}$ $\begin{array}{llll}10 & 23 & 5 \mathrm{~d}^{5} 6 \mathrm{~s} & 54464.11(3)\end{array}$ $\begin{array}{lllllll}-0.002 & 19 & 21 & 5 \mathrm{~d}^{5} 6 \mathrm{~s} & 69741.38(3) & 145003.78(4) & \mathrm{pc}\end{array}$ $\begin{array}{llllllll}0.004 & 8 & 18 & 5 \mathrm{~d}^{5} 6 \mathrm{~s} & 58740.09(6) & 133911.73(6) & \mathrm{pc}\end{array}$ $\begin{array}{lllllll}0.003 & 14 & 25 & 5 \mathrm{~d}^{5} 6 \mathrm{~s} & 66110.16(3) & 141214.36(3) & \mathrm{pc}\end{array}$ $\begin{array}{lllllll}-0.011 & 11 & 9 & 5 \mathrm{~d}^{5} 6 \mathrm{~s} & 81351.39(2) & 156429.22(2) & \mathrm{pc}\end{array}$ $\begin{array}{llllllll}-0.006 & 9 & 22 & 5 \mathrm{~d}^{5} 6 \mathrm{~s} & 76295.33(5) & 151364.78(6) & \mathrm{pc}\end{array}$ $\begin{array}{lllllll}0.016 & 9 & 18 & 5 \mathrm{~d}^{5} 6 \mathrm{~s} & 70298.76(2) & 145359.05(2) & \mathrm{pc} *\end{array}$ $\begin{array}{rrrrrrrr}-0.005 & 8 & 16 & 5 \mathrm{~d}^{5} 6 \mathrm{~s} & 84734.80(4) & 159762.71(5) & \mathrm{pc} *\end{array}$ $\begin{array}{lllllll}0.003 & 14 & 22 & 5 \mathrm{~d}^{5} 6 \mathrm{~s} & 58041.98(4) & 133037.83(3) & \mathrm{pc}\end{array}$ $\begin{array}{rrrrrrr}-0.020 & 17 & 26 & 5 \mathrm{~d}^{5} 6 \mathrm{~s} & 71191.62(4) & 146186.18(3) & \mathrm{pc} *\end{array}$ $\begin{array}{lllllll}0.008 & 6 & 9 & 5 \mathrm{~d}^{5} 6 \mathrm{~s} & 95628.69(5) & 170618.53(5) & \mathrm{pc}\end{array}$ $\begin{array}{lllllll}0.002 & 7 & 9 & 5 \mathrm{~d}^{5} 6 \mathrm{~s} & 85249.67(3) & 160207.91(4) & \mathrm{pc}\end{array}$ $\begin{array}{lllllll}0.005 & 13 & 19 & 5 \mathrm{~d}^{5} 6 \mathrm{~s} & 79208.51(3) & 154152.55(3) & \mathrm{pc}\end{array}$ $\begin{array}{llllllll}0.001 & 11 & 20 & 5 \mathrm{~d}^{5} 6 \mathrm{~s} & 76493.69(3) & 151395.26(3) & \mathrm{pc} *\end{array}$ $\begin{array}{lllllll}0.005 & 12 & 21 & 5 \mathrm{~d}^{5} 6 \mathrm{~s} & 74057.35(4) & 148937.72(5) & \mathrm{pc}\end{array}$ $\begin{array}{lllllll}-0.007 & 16 & 22 & 5 \mathrm{~d}^{5} 6 \mathrm{~s} & 59210.11(5) & 134025.31(4) & \mathrm{pc}\end{array}$ $\begin{array}{lllllll}-0.001 & 16 & 24 & 5 \mathrm{~d}^{5} 6 \mathrm{~s} & 59232.65(3) & 134025.31(4) & \mathrm{pc}\end{array}$ $\begin{array}{lllllll}-0.006 & 6 & 13 & 5 \mathrm{~d}^{5} 6 \mathrm{~s} & 90497.75(4) & 165239.60(5) & \mathrm{pc}\end{array}$ $\begin{array}{llllllll}0.005 & 11 & 14 & 5 \mathrm{~d}^{5} 6 \mathrm{~s} & 82458.31(3) & 157167.25(4) & \mathrm{pc} *\end{array}$ $\begin{array}{lllllll}0.015 & 10 & 22 & 5 \mathrm{~d}^{5} 6 \mathrm{~s} & 76295.33(5) & 151000.14(5) & \mathrm{pc}\end{array}$ $\begin{array}{lllllll}0.001 & 4 & 14 & 5 \mathrm{~d}^{5} 6 \mathrm{~s} & 91240.96(6) & 165923.23(6) & \mathrm{pc}\end{array}$ $\begin{array}{lllllll}0.003 & 8 & 18 & 5 \mathrm{~d}^{5} 6 \mathrm{~s} & 58740.09(6) & 133412.78(5) & \mathrm{pc}\end{array}$ $\begin{array}{lllllll}0.014 & 13 & 19 & 5 \mathrm{~d}^{5} 6 \mathrm{~s} & 68255.33(2) & 142865.10(2) & \mathrm{pc} *\end{array}$ $\begin{array}{lllllll}-0.005 & 10 & 23 & 5 \mathrm{~d}^{5} 6 \mathrm{~s} & 54464.11(3) & 129069.98(3) & \mathrm{pc}\end{array}$ $\begin{array}{lllllll}-0.002 & 12 & 18 & 5 \mathrm{~d}^{5} 6 \mathrm{~s} & 80577.63(6) & 155160.61(5) & \mathrm{pc}\end{array}$ $\begin{array}{lllllll}0.001 & 14 & 23 & 5 \mathrm{~d}^{5} 6 \mathrm{~s} & 69158.48(5) & 143731.85(5) & \mathrm{pc}\end{array}$ $\begin{array}{lllllll}0.003 & 15 & 18 & 5 \mathrm{~d}^{5} 6 \mathrm{~s} & 70298.76(2) & 144855.15(3) & \mathrm{pc} *\end{array}$ $\begin{array}{lllllll}0.010 & 8 & 9 & 5 \mathrm{~d}^{5} 6 \mathrm{~s} & 85249.67(3) & 159791.70(4) & \mathrm{pc} *\end{array}$ $\begin{array}{lllllll}0.016 & 13 & 9 & 5 \mathrm{~d}^{5} 6 \mathrm{~s} & 60272.97(1) & 134707.64(2) & \mathrm{pc} *\end{array}$ $\begin{array}{lllllll}0.012 & 15 & 25 & 5 \mathrm{~d}^{5} 6 \mathrm{~s} & 66110.16(3) & 140526.92(3) & \text { pc }\end{array}$ $\begin{array}{lllllll}0.003 & 5 & 6 & 5 \mathrm{~d}^{5} 6 \mathrm{~s} & 67091.85(1) & 141466.77(2) & \mathrm{pc}\end{array}$ $\begin{array}{rrrrrrr}-0.013 & 10 & 20 & 5 \mathrm{~d}^{6} & 46354.20(4) & 120722.59(4) & \mathrm{pc} *\end{array}$ $\begin{array}{lrlllll}0.010 & 8 & 10 & 5 \mathrm{~d}^{5} 6 \mathrm{~s} & 88009.29(3) & 162374.44(3) & \mathrm{pc}\end{array}$ $\begin{array}{lllllll}-0.004 & 17 & 26 & 5 \mathrm{~d}^{5} 6 \mathrm{~s} & 71191.62(4) & 145525.25(5) & \mathrm{pc}\end{array}$ $\begin{array}{lllllll}0.003 & 12 & 12 & 5 \mathrm{~d}^{5} 6 \mathrm{~s} & 84005.15(4) & 158188.33(4) & \mathrm{pc}\end{array}$ $\begin{array}{lllllll}0.009 & 12 & 19 & 5 \mathrm{~d}^{5} 6 \mathrm{~s} & 79502.00(4) & 153658.98(5) & \mathrm{pc}\end{array}$ $\begin{array}{lllllll}0.008 & 6 & 19 & 5 \mathrm{~d}^{5} 6 \mathrm{~s} & 74545.24(3) & 148685.19(2) & \text { pc }\end{array}$ $\begin{array}{lllllll}0.013 & 9 & 17 & 5 \mathrm{~d}^{5} 6 \mathrm{~s} & 87782.64(4) & 161902.01(4) & \mathrm{pc}\end{array}$ $\begin{array}{lllllll}-0.011 & 10 & 23 & 5 \mathrm{~d}^{5} 6 \mathrm{~s} & 55627.70(2) & 129741.29(3) & \mathrm{pc}\end{array}$ $\begin{array}{lllllll}0.005 & 7 & 9 & 5 \mathrm{~d}^{5} 6 \mathrm{~s} & 95628.69(5) & 169715.71(6) & \mathrm{pc}\end{array}$ $\begin{array}{lllllll}-0.007 & 11 & 21 & 5 \mathrm{~d}^{5} 6 \mathrm{~s} & 59206.99(2) & 133204.10(2) & \text { pc }\end{array}$ $\begin{array}{lllllll}-0.005 & 11 & 14 & 5 d^{5} 6 s & 82458.31(3) & 156429.22(2) & \text { pc }\end{array}$ $\begin{array}{lllllll}-0.006 & 14 & 19 & 5 \mathrm{~d}^{5} 6 \mathrm{~s} & 79502.00(4) & 153412.13(4) & \mathrm{pc}\end{array}$ 


\begin{tabular}{|c|c|c|c|c|c|c|c|c|c|c|c|c|c|c|}
\hline 89 & 88 & 1353.409 & 73887.47 & 1353.3957 & 0.0022 & -0.75 & 0.014 & 15 & 25 & $5 d^{5} 6 s$ & $66110.16(3)$ & $139998.38(4)$ & $\mathrm{pc} *$ & \\
\hline 311 & 39 & 1353.884 & 73861.58 & 1353.8798 & 0.0040 & -0.22 & 0.004 & 8 & 9 & $5 d^{5} 6 s$ & $95628.69(5)$ & $169490.49(5)$ & pc & \\
\hline 55 & 56 & 1354.443 & 73831.07 & 1354.4475 & 0.0023 & 0.23 & -0.004 & 14 & 21 & $5 d^{5} 6 s$ & $59206.99(2)$ & $133037.83(3)$ & pc & \\
\hline 79 & 58 & 1354.667 & 73818.88 & 1354.6642 & 0.0030 & -0.15 & 0.003 & 9 & 11 & $5 d^{5} 6 s$ & $75494.15(7)$ & $149313.18(6)$ & pc & \\
\hline 346 & 333 & 1354.791 & 73812.13 & 1354.7903 & 0.0020 & -0.03 & 0.000 & 19 & 26 & $5 d^{5} 6 s$ & $71191.62(4)$ & $145003.78(4)$ & $\mathrm{pc}$ & \\
\hline 8 & 34 & 1355.241 & 73787.64 & 1355.2339 & 0.0030 & -0.36 & 0.007 & 12 & 20 & $5 d^{5} 6 s$ & $63912.64(4)$ & $137700.64(3)$ & pc & \\
\hline 526 & 885 & 1355.508 & 73773.09 & 1355.5051 & 0.0024 & -0.15 & 0.003 & 11 & 20 & $5 d^{5} 6 s$ & $63912.64(4)$ & $137685.88(5)$ & $\mathrm{pc}$ & \\
\hline 93 & 61 & 1356.236 & 73733.45 & 1356.2332 & 0.0040 & -0.18 & 0.003 & 13 & 9 & $5 d^{5} 6 s$ & $81351.39(2)$ & $155085.02(3)$ & pc & \\
\hline 449 & 324 & 1356.557 & 73716.04 & 1356.5561 & 0.0030 & -0.04 & 0.001 & 7 & 18 & $5 d^{5} 6 s$ & $80577.63(6)$ & $154293.71(6)$ & pc & \\
\hline 137 & 70 & 1356.746 & 73705.77 & 1356.7440 & 0.0030 & -0.10 & 0.002 & 11 & 21 & $5 d^{5} 6 s$ & $74057.35(4)$ & $147763.22(4)$ & pc & \\
\hline 232 & 46 & 1357.135 & 73684.66 & 1357.1405 & 0.0060 & 0.32 & -0.006 & 7 & 2 & $5 d^{5} 6 s$ & $91225.56(2)$ & $164909.90(2)$ & pc & \\
\hline 95 & 99 & 1357.522 & 73663.65 & 1357.5239 & 0.0024 & 0.12 & -0.002 & 15 & 26 & $5 d^{5} 6 s$ & $71191.62(4)$ & $144855.15(3)$ & pc & \\
\hline 121 & 34 & 1359.526 & 73555.03 & 1359.5282 & 0.0030 & 0.10 & -0.002 & 12 & 20 & $5 d^{5} 6 s$ & $81605.68(5)$ & $155160.61(5)$ & $\mathrm{pc}$ & \\
\hline 176 & 206 & 1360.469 & 73504.07 & 1360.4750 & 0.0030 & 0.33 & -0.006 & 10 & 23 & $5 d^{5} 6 s$ & $69158.48(5)$ & $142662.22(6)$ & $\mathrm{pc}$ & \\
\hline 264 & 135 & 1360.908 & 73480.33 & 1360.9095 & 0.0030 & 0.06 & -0.001 & 8 & 19 & $5 d^{5} 6 s$ & $79502.00(4)$ & $152982.27(4)$ & pc & \\
\hline 445 & 159 & 1361.215 & 73463.77 & 1361.2092 & 0.0063 & -0.32 & 0.006 & 5 & 2 & $5 d^{5} 6 s$ & $91225.56(2)$ & $164689.65(3)$ & pc & \\
\hline 12 & 26 & 1362.733 & 73381.92 & 1362.7435 & 0.0050 & 0.54 & -0.010 & 10 & 6 & $5 d^{5} 6 s$ & $67091.85(1)$ & $140473.23(2)$ & $\mathrm{pc} *$ & \\
\hline 116 & 82 & 1363.059 & 73364.38 & 1363.0530 & 0.0030 & -0.34 & 0.006 & 9 & 20 & $5 d^{5} 6 s$ & $63912.64(4)$ & $137277.36(4)$ & $\mathrm{pc}$ & \\
\hline 299 & 271 & 1363.526 & 73339.25 & 1363.5228 & 0.0030 & -0.19 & 0.003 & 11 & 19 & $5 d^{5} 6 s$ & $74545.24(3)$ & $147884.68(4)$ & pc & \\
\hline 144 & 56 & 1366.223 & 73194.48 & 1366.2218 & 0.0040 & -0.08 & 0.002 & 8 & 15 & $5 d^{5} 6 s$ & $75164.22(2)$ & $148358.78(3)$ & pc & \\
\hline 69 & 35 & 1366.773 & 73165.02 & 1366.7715 & 0.0040 & -0.10 & 0.002 & 13 & 19 & $5 d^{5} 6 s$ & $79208.51(3)$ & $152373.63(3)$ & pc & \\
\hline 53 & 620 & 1367.549 & 73123.51 & 1367.5453 & 0.0031 & -0.21 & 0.004 & 13 & 21 & $5 d^{5} 6 s$ & $69741.38(3)$ & $142865.10(2)$ & pc & 100, Ir III \\
\hline 367 & 336 & 1369.530 & 73017.72 & 1369.5281 & 0.0020 & -0.13 & 0.002 & 9 & 22 & $5 d^{5} 6 s$ & $76295.33(5)$ & $149313.18(6)$ & pc & \\
\hline 38 & 41 & 1370.397 & 72971.54 & 1370.3984 & 0.0042 & 0.06 & -0.001 & 8 & 15 & $5 d^{5} 6 s$ & $75164.22(2)$ & $148135.70(2)$ & pc & \\
\hline 18 & 27 & 1370.517 & 72965.18 & 1370.5214 & 0.0020 & 0.25 & -0.005 & 15 & 16 & $5 d^{6}$ & $52259.88(3)$ & $125224.81(4)$ & pc & \\
\hline 27 & 54 & 1372.144 & 72878.66 & 1372.1549 & 0.0023 & 0.59 & -0.011 & 15 & 22 & $5 d^{5} 6 s$ & $59210.11(5)$ & $132088.18(4)$ & pc & \\
\hline 64 & 68 & 1373.847 & 72788.32 & 1373.8538 & 0.0031 & 0.37 & -0.007 & 17 & 20 & $5 d^{5} 6 s$ & $72737.30(6)$ & $145525.25(5)$ & pc & \\
\hline 21 & 208 & 1373.931 & 72783.88 & 1373.9257 & 0.0023 & -0.26 & 0.005 & 13 & 14 & $5 d^{5} 6 s$ & $50417.92(2)$ & $123202.06(3)$ & pc & \\
\hline 150 & 180 & 1374.175 & 72770.93 & 1374.1768 & 0.0050 & 0.10 & -0.002 & 8 & 20 & $5 d^{5} 6 s$ & $76493.69(3)$ & $149264.53(2)$ & $\mathrm{pc}$ & \\
\hline 455 & 1149 & 1374.691 & 72743.60 & 1374.6413 & 0.0030 & -2.65 & 0.050 & 12 & 16 & $5 d^{5} 6 s$ & $84734.80(4)$ & $157481.05(5)$ & $\mathrm{pc} *$ & $200, \mathrm{~m}(\mathrm{C}$ II $)$ \\
\hline 223 & 1149 & 1374.691 & 72743.60 & 1374.7027 & 0.0043 & 0.60 & -0.011 & 7 & 13 & $5 d^{5} 6 s$ & $90497.75(4)$ & $163240.75(5)$ & $\mathrm{pc} *$ & $300, \mathrm{~m}(\mathrm{C}$ II $)$ \\
\hline 706 & 847 & 1377.131 & 72614.74 & 1377.1305 & 0.0040 & -0.02 & 0.000 & 6 & 18 & $5 d^{5} 6 s$ & $80577.63(6)$ & $153192.39(7)$ & $\mathrm{pc}$ & \\
\hline 211 & 434 & 1378.544 & 72540.31 & 1378.5454 & 0.0025 & 0.08 & -0.002 & 14 & 26 & $5 d^{5} 6 s$ & $71191.62(4)$ & $143731.85(5)$ & pc & \\
\hline 210 & 33 & 1378.723 & 72530.88 & 1378.7261 & 0.0030 & 0.16 & -0.003 & 11 & 13 & $5 d^{5} 6 s$ & $90123.22(5)$ & $162653.94(5)$ & pc & \\
\hline 185 & 261 & 1378.995 & 72516.59 & 1378.9937 & 0.0030 & -0.06 & 0.001 & 12 & 20 & $5 d^{5} 6 s$ & 63912.64(4) & $136429.29(5)$ & $\mathrm{pc}$ & \\
\hline 38 & 94 & 1379.263 & 72502.47 & 1379.2647 & 0.0031 & 0.07 & -0.001 & 8 & 25 & $5 d^{5} 6 s$ & $66110.16(3)$ & $138612.56(2)$ & $\mathrm{pc}$ & \\
\hline 31 & 28 & 1380.030 & 72462.21 & 1380.0194 & 0.0040 & -0.54 & 0.010 & 7 & 6 & $5 d^{5} 6 s$ & $71884.65(1)$ & $144347.40(1)$ & $\mathrm{pc} *$ & \\
\hline 21 & 86 & 1380.119 & 72457.49 & 1380.1213 & 0.0023 & 0.09 & -0.002 & 13 & 21 & $5 d^{5} 6 s$ & $69741.38(3)$ & $142198.78(4)$ & $\mathrm{pc}$ & \\
\hline 15 & 151 & 1382.583 & 72328.38 & 1382.5833 & 0.0020 & 0.01 & 0.000 & 9 & 14 & $5 d^{5} 6 s$ & $50417.92(2)$ & $122746.29(2)$ & pc & \\
\hline 102 & 62 & 1383.668 & 72271.65 & 1383.6696 & 0.0031 & 0.06 & -0.001 & 15 & 19 & $5 d^{5} 6 s$ & $68255.33(2)$ & $140526.92(3)$ & pc & \\
\hline 78 & 92 & 1384.699 & 72217.85 & 1384.6983 & 0.0030 & -0.05 & 0.001 & 10 & 19 & $5 d^{5} 6 s$ & $68255.33(2)$ & $140473.23(2)$ & pc & \\
\hline 29 & 63 & 1385.107 & 72196.61 & 1385.1066 & 0.0030 & 0.00 & 0.000 & 8 & 23 & $5 d^{5} 6 s$ & $55627.70(2)$ & 127824.31(1) & $\mathrm{pc}$ & \\
\hline 288 & 118 & 1387.868 & 72052.94 & 1387.8615 & 0.0030 & -0.36 & 0.007 & 12 & 20 & $5 d^{5} 6 s$ & $81605.68(5)$ & $153658.98(5)$ & $\mathrm{pc}$ & \\
\hline 16 & 29 & 1388.245 & 72033.40 & 1388.2337 & 0.0040 & -0.59 & 0.011 & 8 & 5 & $5 d^{5} 6 s$ & $65108.69(0)$ & $137142.67(1)$ & $\mathrm{pc} *$ & \\
\hline
\end{tabular}




$\begin{array}{rrr}156 & 150 & 1389.224 \\ 53 & 44 & 1390.128 \\ 206 & 51 & 1391.273 \\ 56 & 75 & 1393.243 \\ 122 & 153 & 1395.839 \\ 101 & 153 & 1395.839 \\ 137 & 190 & 1398.643 \\ 128 & 190 & 1398.643 \\ 88 & 220 & 1398.909 \\ 224 & 49 & 1399.011 \\ 385 & 533 & 1399.128 \\ 177 & 128 & 1399.224 \\ 146 & 40 & 1400.300 \\ 94 & 41 & 1400.737 \\ 53 & 70 & 1400.899 \\ 125 & 34 & 1403.127 \\ 62 & 43 & 1406.860 \\ 89 & 21 & 1407.917 \\ 80 & 119 & 1408.011 \\ 160 & 119 & 1408.011 \\ 177 & 81 & 1409.513 \\ 24 & 26 & 1409.607 \\ 29 & 15 & 1412.467 \\ 35 & 19 & 1412.715 \\ 97 & 469 & 1413.217 \\ 119 & 108 & 1416.808 \\ 40 & 58 & 1418.264 \\ 64 & 42 & 1419.269 \\ 20 & 22 & 1419.353 \\ 25 & 32 & 1419.412 \\ 287 & 960 & 1419.992 \\ 44 & 97 & 1421.449 \\ 8 & 20 & 1421.601 \\ 28 & 42 & 1422.200 \\ 80 & 50 & 1422.994 \\ 412 & 844 & 1423.342 \\ 208 & 58 & 1424.928 \\ 55 & 40 & 1425.008 \\ 28 & 31 & 1426.286 \\ 103 & 70 & 1429.601 \\ 95 & 187 & 1429.865 \\ 60 & 75 & 1431.923 \\ 22 & 29 & 1433.509 \\ 74 & 119 & 1434.514\end{array}$

$\begin{array}{lllll}71982.64 & 1389.2231 & 0.0030 & -0.04\end{array}$ $\begin{array}{lllll}71935.81 & 1390.1265 & 0.0023 & -0.09\end{array}$ $\begin{array}{lllll}71876.63 & 1391.2716 & 0.0050 & -0.06\end{array}$ $\begin{array}{lllll}71775.00 & 1393.2399 & 0.0030 & -0.15\end{array}$ $\begin{array}{llll}71641.50 & 1395.8499 & 0.0032 & 0.56\end{array}$ $\begin{array}{llll}71641.50 & 1395.8291 & 0.0041 & -0.51\end{array}$ $\begin{array}{lllll}71497.89 & 1398.6495 & 0.0030 & 0.35\end{array}$ $\begin{array}{lllll}71497.89 & 1398.6378 & 0.0041 & -0.25\end{array}$ $\begin{array}{llll}71484.29 & 1398.9072 & 0.0030 & -0.08\end{array}$ $\begin{array}{llll}71479.06 & 1399.0193 & 0.0040 & 0.42\end{array}$ $\begin{array}{lllll}71473.07 & 1399.1301 & 0.0030 & 0.09\end{array}$ $\begin{array}{lllll}71468.16 & 1399.2296 & 0.0031 & 0.26\end{array}$ $\begin{array}{lllll}71413.27 & 1400.3057 & 0.0030 & 0.29\end{array}$ $\begin{array}{llll}71391.01 & 1400.7370 & 0.0030 & 0.02\end{array}$ $\begin{array}{llll}71382.75 & 1400.9016 & 0.0030 & 0.15\end{array}$ $\begin{array}{rrrr}71269.40 & 1403.1242 & 0.0030 & -0.13\end{array}$ $\begin{array}{lllll}71080.30 & 1406.8681 & 0.0050 & 0.43\end{array}$ $\begin{array}{lllll}71026.93 & 1407.9236 & 0.0040 & 0.35\end{array}$ $\begin{array}{lllll}71022.18 & 1408.0152 & 0.0041 & 0.22\end{array}$ $\begin{array}{lllll}71022.18 & 1408.0096 & 0.0040 & -0.06\end{array}$ $\begin{array}{llll}70946.47 & 1409.5142 & 0.0023 & 0.04\end{array}$ $\begin{array}{lllll}70941.77 & 1409.6052 & 0.0030 & -0.08\end{array}$ $\begin{array}{llll}70798.11 & 1412.4733 & 0.0030 & 0.31\end{array}$ $\begin{array}{lllll}70785.67 & 1412.7179 & 0.0030 & 0.13\end{array}$ $\begin{array}{lllll}70760.53 & 1413.2138 & 0.0021 & -0.17\end{array}$ $\begin{array}{lllll}70581.18 & 1416.8053 & 0.0021 & -0.15\end{array}$ $\begin{array}{llll}70508.71 & 1418.2659 & 0.0021 & 0.08\end{array}$ $\begin{array}{lllll}70458.80 & 1419.2744 & 0.0024 & 0.26\end{array}$ $\begin{array}{lllll}70454.64 & 1419.3437 & 0.0030 & -0.46\end{array}$ $\begin{array}{lllll}70451.71 & 1419.4216 & 0.0023 & 0.48\end{array}$ $\begin{array}{lllll}70422.92 & 1420.0005 & 0.0040 & 0.41\end{array}$ $\begin{array}{lllll}70350.73 & 1421.4597 & 0.0050 & 0.51\end{array}$ $\begin{array}{lllll}70343.24 & 1421.5924 & 0.0030 & -0.41\end{array}$ $\begin{array}{llll}70313.60 & 1422.2087 & 0.0031 & 0.43\end{array}$ $\begin{array}{lllll}70274.35 & 1422.9917 & 0.0030 & -0.12\end{array}$ $\begin{array}{lllll}70257.16 & 1423.3457 & 0.0024 & 0.16\end{array}$ $\begin{array}{lllll}70178.98 & 1424.9269 & 0.0030 & -0.06\end{array}$ $\begin{array}{rrrrr}70175.05 & 1425.0197 & 0.0041 & 0.58\end{array}$ $\begin{array}{lllll}70112.19 & 1426.2757 & 0.0023 & -0.48\end{array}$ $\begin{array}{lllll}69949.57 & 1429.6020 & 0.0030 & 0.03\end{array}$ $\begin{array}{lllll}69936.66 & 1429.8734 & 0.0030 & 0.40\end{array}$ $\begin{array}{lllll}69836.17 & 1431.9115 & 0.0040 & -0.55\end{array}$ $\begin{array}{lllll}69758.87 & 1433.5047 & 0.0031 & -0.23\end{array}$ $\begin{array}{lllll}69710.00 & 1434.5111 & 0.0040 & -0.16\end{array}$ $\begin{array}{lllllll}0.001 & 15 & 23 & 5 \mathrm{~d}^{5} 6 \mathrm{~s} & 69158.48(5) & 141141.16(4) & \mathrm{pc}\end{array}$ $\begin{array}{lllllll}0.002 & 19 & 19 & 5 \mathrm{~d}^{5} 6 \mathrm{~s} & 73067.88(5) & 145003.78(4) & \mathrm{pc}\end{array}$ $\begin{array}{lllllll}0.001 & 8 & 13 & 5 \mathrm{~d}^{5} 6 \mathrm{~s} & 90497.75(4) & 162374.44(3) & \mathrm{pc}\end{array}$ $\begin{array}{lllllll}0.003 & 14 & 20 & 5 \mathrm{~d}^{5} 6 \mathrm{~s} & 72737.30(6) & 144512.45(5) & \mathrm{pc}\end{array}$ $\begin{array}{lllllll}-0.011 & 17 & 19 & 5 \mathrm{~d}^{5} 6 \mathrm{~s} & 74545.24(3) & 146186.18(3) & \mathrm{pc}\end{array}$ $\begin{array}{lllllll}0.010 & 8 & 20 & 5 \mathrm{~d}^{5} 6 \mathrm{~s} & 76493.69(3) & 148135.70(2) & \mathrm{pc}\end{array}$ $\begin{array}{llllllll}-0.007 & 11 & 19 & 5 \mathrm{~d}^{5} 6 \mathrm{~s} & 79208.51(3) & 150706.05(4) & \mathrm{pc}\end{array}$ $\begin{array}{lllllll}0.005 & 10 & 19 & 5 \mathrm{~d}^{5} 6 \mathrm{~s} & 79502.00(4) & 151000.14(5) & \mathrm{pc}\end{array}$ $\begin{array}{lllllll}0.002 & 14 & 14 & 5 \mathrm{~d}^{5} 6 \mathrm{~s} & 50417.92(2) & 121902.29(3) & \mathrm{pc}\end{array}$ $\begin{array}{lllllll}-0.008 & 7 & 9 & 5 \mathrm{~d}^{5} 6 \mathrm{~s} & 95628.69(5) & 167107.33(4) & \mathrm{pc}\end{array}$ $\begin{array}{lllllll}-0.002 & 14 & 21 & 5 \mathrm{~d}^{5} 6 \mathrm{~s} & 69741.38(3) & 141214.36(3) & \mathrm{pc}\end{array}$ $\begin{array}{lllllll}-0.005 & 17 & 21 & 5 \mathrm{~d}^{5} 6 \mathrm{~s} & 74057.35(4) & 145525.25(5) & \text { pc }\end{array}$ $\begin{array}{lllllll}-0.006 & 11 & 14 & 5 \mathrm{~d}^{5} 6 \mathrm{~s} & 91240.96(6) & 162653.94(5) & \mathrm{pc}\end{array}$ $\begin{array}{llllllll}0.000 & 11 & 20 & 5 \mathrm{~d}^{5} 6 \mathrm{~s} & 76493.69(3) & 147884.68(4) & \mathrm{pc}\end{array}$ $\begin{array}{lllllll}-0.003 & 16 & 21 & 5 \mathrm{~d}^{5} 6 \mathrm{~s} & 74057.35(4) & 145439.95(4) & \mathrm{pc}\end{array}$ $\begin{array}{llllllll}0.003 & 11 & 20 & 5 \mathrm{~d}^{5} 6 \mathrm{~s} & 76493.69(3) & 147763.22(4) & \mathrm{pc}\end{array}$ $\begin{array}{lllllll}-0.009 & 13 & 12 & 5 \mathrm{~d}^{5} 6 \mathrm{~s} & 84005.15(4) & 155085.02(3) & \mathrm{pc} *\end{array}$ $\begin{array}{lllllll}-0.007 & 11 & 19 & 5 \mathrm{~d}^{5} 6 \mathrm{~s} & 79208.51(3) & 150235.09(3) & \mathrm{pc} *\end{array}$ $\begin{array}{lllllll}-0.004 & 17 & 15 & 5 \mathrm{~d}^{5} 6 \mathrm{~s} & 75164.22(2) & 146186.18(3) & \mathrm{pc}\end{array}$ $\begin{array}{lllllll}0.001 & 13 & 9 & 5 d^{5} 6 \mathrm{~s} & 81351.39(2) & 152373.63(3) & \mathrm{pc}\end{array}$ $\begin{array}{lllllll}-0.001 & 19 & 21 & 5 \mathrm{~d}^{5} 6 \mathrm{~s} & 74057.35(4) & 145003.78(4) & \mathrm{pc}\end{array}$ $\begin{array}{lllllll}0.002 & 8 & 14 & 5 \mathrm{~d}^{5} 6 \mathrm{~s} & 50417.92(2) & 121359.77(1) & \mathrm{pc}\end{array}$ $\begin{array}{lllllll}-0.006 & 15 & 21 & 5 \mathrm{~d}^{5} 6 \mathrm{~s} & 74057.35(4) & 144855.15(3) & \mathrm{pc} *\end{array}$ $\begin{array}{lllllll}-0.003 & 15 & 21 & 5 \mathrm{~d}^{5} 6 \mathrm{~s} & 69741.38(3) & 140526.92(3) & \mathrm{pc} *\end{array}$ $\begin{array}{lllllll}0.003 & 15 & 23 & 5 \mathrm{~d}^{5} 6 \mathrm{~s} & 54464.11(3) & 125224.81(4) & \text { pc }\end{array}$ $\begin{array}{lllllll}0.003 & 16 & 23 & 5 \mathrm{~d}^{5} 6 \mathrm{~s} & 69158.48(5) & 139739.81(5) & \text { pc }\end{array}$ $\begin{array}{lllllll}-0.002 & 10 & 24 & 5 \mathrm{~d}^{5} 6 \mathrm{~s} & 59232.65(3) & 129741.29(3) & \mathrm{pc}\end{array}$ $\begin{array}{lllllll}-0.005 & 19 & 19 & 5 \mathrm{~d}^{5} 6 \mathrm{~s} & 74545.24(3) & 145003.78(4) & \mathrm{pc}\end{array}$ $\begin{array}{lllllll}0.009 & 14 & 21 & 5 \mathrm{~d}^{5} 6 \mathrm{~s} & 74057.35(4) & 144512.45(5) & \mathrm{pc} *\end{array}$ $\begin{array}{llllllll}-0.010 & 14 & 22 & 5 \mathrm{~d}^{5} 6 \mathrm{~s} & 58041.98(4) & 128493.21(4) & \mathrm{pc} *\end{array}$ $\begin{array}{lllllll}-0.008 & 10 & 18 & 5 d^{5} 6 s & 80577.63(6) & 151000.14(5) & \text { pc }\end{array}$ $\begin{array}{lllllll}-0.010 & 13 & 16 & 5 \mathrm{~d}^{5} 6 \mathrm{~s} & 84734.80(4) & 155085.02(3) & \mathrm{pc} *\end{array}$ $\begin{array}{lllllll}0.008 & 13 & 7 & 5 \mathrm{~d}^{6} & 52858.41(2) & 123202.06(3) & \mathrm{pc} *\end{array}$ $\begin{array}{llllllll}-0.009 & 11 & 21 & 5 \mathrm{~d}^{5} 6 \mathrm{~s} & 69741.38(3) & 140054.55(3) & \mathrm{pc} *\end{array}$ $\begin{array}{llllllll}0.003 & 10 & 19 & 5 \mathrm{~d}^{5} 6 \mathrm{~s} & 68255.33(2) & 138529.81(3) & \mathrm{pc}\end{array}$ $\begin{array}{lllllll}-0.003 & 15 & 21 & 5 \mathrm{~d}^{5} 6 \mathrm{~s} & 69741.38(3) & 139998.38(4) & \mathrm{pc}\end{array}$ $\begin{array}{lllllll}0.001 & 12 & 10 & 5 \mathrm{~d}^{5} 6 \mathrm{~s} & 88009.29(3) & 158188.33(4) & \mathrm{pc}\end{array}$ $\begin{array}{lllllll}-0.012 & 10 & 18 & 5 \mathrm{~d}^{5} 6 \mathrm{~s} & 70298.76(2) & 140473.23(2) & \mathrm{pc}\end{array}$ $\begin{array}{lllllll}0.010 & 16 & 20 & 5 \mathrm{~d}^{5} 6 \mathrm{~s} & 63912.64(4) & 134025.31(4) & \mathrm{pc} *\end{array}$ $\begin{array}{llllllll}-0.001 & 15 & 26 & 5 \mathrm{~d}^{5} 6 \mathrm{~s} & 71191.62(4) & 141141.16(4) & \mathrm{pc}\end{array}$ $\begin{array}{lllllll}-0.008 & 5 & 23 & 5 \mathrm{~d}^{5} 6 \mathrm{~s} & 69158.48(5) & 139094.74(6) & \mathrm{pc}\end{array}$ $\begin{array}{lllllll}0.011 & 6 & 20 & 5 \mathrm{~d}^{5} 6 \mathrm{~s} & 76493.69(3) & 146330.41(2) & \mathrm{pc}\end{array}$ $\begin{array}{lllllll}0.005 & 9 & 20 & 5 \mathrm{~d}^{5} 6 \mathrm{~s} & 81605.68(5) & 151364.78(6) & \mathrm{pc} *\end{array}$ $\begin{array}{lllllll}0.003 & 7 & 13 & 5 \mathrm{~d}^{5} 6 \mathrm{~s} & 90497.75(4) & 160207.91(4) & \text { pc }\end{array}$ 


$\begin{array}{rrr}148 & 40 & 1434.752 \\ 100 & 81 & 1435.244 \\ 255 & 143 & 1435.673 \\ 182 & 287 & 1436.911 \\ 28 & 47 & 1439.158 \\ 20 & 12 & 1442.256 \\ 58 & 83 & 1442.793 \\ 315 & 1601 & 1443.821 \\ 312 & 748 & 1444.466 \\ 62 & 38 & 1445.440 \\ 87 & 50 & 1446.242 \\ 49 & 39 & 1447.157 \\ 443 & 1050 & 1447.509 \\ 49 & 72 & 1448.380 \\ 275 & 858 & 1450.060 \\ 36 & 201 & 1450.409 \\ 37 & 43 & 1451.313 \\ 67 & 78 & 1451.651 \\ 161 & 516 & 1452.159 \\ 38 & 33 & 1452.291 \\ 47 & 36 & 1453.344 \\ 82 & 86 & 1454.587 \\ 81 & 558 & 1454.804 \\ 77 & 139 & 1454.842 \\ 32 & 45 & 1455.424 \\ 95 & 134 & 1456.085 \\ 56 & 159 & 1457.357 \\ 588 & 1811 & 1457.771 \\ 50 & 58 & 1458.837 \\ 42 & 41 & 1459.273 \\ 18 & 368 & 1460.630 \\ 202 & 1247 & 1462.662 \\ 227 & 307 & 1462.806 \\ 189 & 311 & 1463.707 \\ 26 & 61 & 1463.832 \\ 39 & 154 & 1464.509 \\ 63 & 68 & 1465.262 \\ 62 & 28 & 1467.530 \\ 47 & 186 & 1467.729 \\ 124 & 168 & 1468.025 \\ 289 & 1356 & 1468.212 \\ 39 & 33 & 1469.008 \\ 80 & 592 & 1471.479 \\ 140 & 799 & 1471.886\end{array}$

$\begin{array}{llll}69698.45 & 1434.7530 & 0.0030 & 0.04\end{array}$ $\begin{array}{llll}69674.57 & 1435.2453 & 0.0030 & 0.07\end{array}$ $\begin{array}{lllll}69653.75 & 1435.6712 & 0.0030 & -0.08\end{array}$ $\begin{array}{lllll}69593.75 & 1436.8985 & 0.0030 & -0.59\end{array}$ $\begin{array}{lllll}69485.09 & 1439.1537 & 0.0020 & -0.19\end{array}$ $\begin{array}{lllll}69335.81 & 1442.2668 & 0.0030 & 0.51\end{array}$ $\begin{array}{llll}69309.99 & 1442.7960 & 0.0030 & 0.12\end{array}$ $\begin{array}{lllll}69260.66 & 1443.8232 & 0.0021 & 0.10\end{array}$ $\begin{array}{lllll}69229.75 & 1444.4622 & 0.0030 & -0.17\end{array}$ $\begin{array}{lllll}69183.10 & 1445.4380 & 0.0050 & -0.08\end{array}$ $\begin{array}{lllll}69144.72 & 1446.2441 & 0.0030 & 0.10\end{array}$ $\begin{array}{lllll}69101.02 & 1447.1703 & 0.0041 & 0.65\end{array}$ $\begin{array}{lllll}69084.20 & 1447.5075 & 0.0040 & -0.07\end{array}$ $\begin{array}{lllll}69042.65 & 1448.3728 & 0.0040 & -0.35\end{array}$ $\begin{array}{lllll}68962.67 & 1450.0586 & 0.0025 & -0.06\end{array}$ $\begin{array}{lllll}68946.07 & 1450.4050 & 0.0030 & -0.20\end{array}$ $\begin{array}{lllll}68903.14 & 1451.3181 & 0.0051 & 0.26\end{array}$ $\begin{array}{lllll}68887.10 & 1451.6455 & 0.0040 & -0.24\end{array}$ $\begin{array}{lllll}68862.96 & 1452.1601 & 0.0024 & 0.03\end{array}$ $\begin{array}{lllll}68856.73 & 1452.2898 & 0.0050 & -0.05\end{array}$ $\begin{array}{llll}68806.83 & 1453.3456 & 0.0021 & 0.07\end{array}$ $\begin{array}{lllll}68748.03 & 1454.5821 & 0.0041 & -0.24\end{array}$ $\begin{array}{llll}68737.79 & 1454.8004 & 0.0023 & -0.16\end{array}$ $\begin{array}{lllll}68735.97 & 1454.8512 & 0.0030 & 0.42\end{array}$ $\begin{array}{lllll}68708.51 & 1455.4251 & 0.0024 & 0.06\end{array}$ $\begin{array}{lllll}68677.30 & 1456.0846 & 0.0040 & -0.03\end{array}$ $\begin{array}{lllll}68617.36 & 1457.3580 & 0.0030 & 0.04\end{array}$ $\begin{array}{llll}68597.90 & 1457.7765 & 0.0040 & 0.28\end{array}$ $\begin{array}{lllll}68547.76 & 1458.8277 & 0.0022 & -0.43\end{array}$ $\begin{array}{lllll}68527.29 & 1459.2703 & 0.0031 & -0.11\end{array}$ $\begin{array}{llll}68463.62 & 1460.6492 & 0.0032 & 0.91\end{array}$ $\begin{array}{lllll}68368.48 & 1462.6651 & 0.0030 & 0.13\end{array}$ $\begin{array}{lllll}68361.74 & 1462.8125 & 0.0030 & 0.28\end{array}$ $\begin{array}{lllll}68319.70 & 1463.7032 & 0.0041 & -0.16\end{array}$ $\begin{array}{llll}68313.83 & 1463.8331 & 0.0030 & 0.03\end{array}$ $\begin{array}{lllll}68282.27 & 1464.5109 & 0.0020 & 0.09\end{array}$ $\begin{array}{lllll}68247.19 & 1465.2558 & 0.0041 & -0.28\end{array}$ $\begin{array}{llll}68141.70 & 1467.5360 & 0.0030 & 0.27\end{array}$ $\begin{array}{lllll}68132.48 & 1467.7292 & 0.0030 & 0.02\end{array}$ $\begin{array}{lllll}68118.72 & 1468.0218 & 0.0030 & -0.16\end{array}$ $\begin{array}{lllll}68110.04 & 1468.2136 & 0.0031 & 0.06\end{array}$ $\begin{array}{lllll}68073.13 & 1469.0052 & 0.0030 & -0.15\end{array}$ $\begin{array}{lllll}67958.83 & 1471.4698 & 0.0030 & -0.43\end{array}$ $\begin{array}{lllll}67940.05 & 1471.8835 & 0.0030 & -0.11\end{array}$ $\begin{array}{lllllll}-0.001 & 12 & 17 & 5 d^{5} 6 s & 87782.64(4) & 157481.05(5) & \text { pc }\end{array}$ $\begin{array}{lllllll}-0.002 & 14 & 21 & 5 d^{5} 6 \mathrm{~s} & 74057.35(4) & 143731.85(5) & \mathrm{pc}\end{array}$ $\begin{array}{lllllll}0.002 & 12 & 12 & 5 \mathrm{~d}^{5} 6 \mathrm{~s} & 84005.15(4) & 153658.98(5) & \text { pc }\end{array}$ $\begin{array}{lllllll}0.012 & 10 & 19 & 5 \mathrm{~d}^{5} 6 \mathrm{~s} & 73067.88(5) & 142662.22(6) & \mathrm{pc}\end{array}$ $\begin{array}{lllllll}0.004 & 17 & 25 & 5 \mathrm{~d}^{5} 6 \mathrm{~s} & 66110.16(3) & 135595.44(4) & \mathrm{pc}\end{array}$ $\begin{array}{lllllll}-0.011 & 15 & 26 & 5 d^{5} 6 s & 71191.62(4) & 140526.92(3) & \text { pc * }\end{array}$ $\begin{array}{lllllll}-0.002 & 15 & 25 & 5 d^{5} 6 s & 66110.16(3) & 135420.03(3) & \text { pc }\end{array}$ $\begin{array}{lllllll}-0.002 & 14 & 24 & 5 d^{5} 6 s & 59232.65(3) & 128493.21(4) & \text { pc }\end{array}$ $\begin{array}{lllllll}0.004 & 17 & 22 & 5 d^{5} 6 s & 76295.33(5) & 145525.25(5) & \text { pc }\end{array}$ $\begin{array}{lllllll}0.002 & 7 & 15 & 5 \mathrm{~d}^{5} 6 \mathrm{~s} & 75164.22(2) & 144347.40(1) & \mathrm{pc}\end{array}$ $\begin{array}{lllllll}-0.002 & 16 & 22 & 5 d^{5} 6 s & 76295.33(5) & 145439.95(4) & \text { pc }\end{array}$ $\begin{array}{lllllll}-0.014 & 11 & 20 & 5 \mathrm{~d}^{5} 6 \mathrm{~s} & 81605.68(5) & 150706.05(4) & \mathrm{pc} *\end{array}$ $\begin{array}{lllllll}0.001 & 5 & 18 & 5 \mathrm{~d}^{5} 6 \mathrm{~s} & 80577.63(6) & 149661.90(7) & \mathrm{pc}\end{array}$ $\begin{array}{lllllll}0.007 & 7 & 20 & 5 d^{5} 6 s & 76493.69(3) & 145536.69(3) & \text { pc }\end{array}$ $\begin{array}{lllllll}0.001 & 10 & 23 & 5 \mathrm{~d}^{5} 6 \mathrm{~s} & 69158.48(5) & 138121.21(6) & \text { pc }\end{array}$ $\begin{array}{lllllll}0.004 & 16 & 20 & 5 \mathrm{~d}^{5} 6 \mathrm{~s} & 76493.69(3) & 145439.95(4) & \mathrm{pc} *\end{array}$ $\begin{array}{lllllll}-0.005 & 13 & 9 & 5 d^{5} 6 s & 85249.67(3) & 154152.55(3) & \text { pc* }\end{array}$ $\begin{array}{lllllll}0.005 & 8 & 19 & 5 \mathrm{~d}^{5} 6 \mathrm{~s} & 68255.33(2) & 137142.67(1) & \mathrm{pc}\end{array}$ $\begin{array}{lllllll}0.001 & 11 & 26 & 5 d^{5} 6 s & 71191.62(4) & 140054.55(3) & \text { pc }\end{array}$ $\begin{array}{llllllll}0.001 & 8 & 19 & 5 \mathrm{~d}^{5} 6 \mathrm{~s} & 79502.00(4) & 148358.78(3) & \mathrm{pc} *\end{array}$ $\begin{array}{lllllll}-0.002 & 15 & 26 & 5 d^{5} 6 s & 71191.62(4) & 139998.38(4) & \text { pc }\end{array}$ $\begin{array}{lllllll}0.005 & 11 & 10 & 5 \mathrm{~d}^{5} 6 \mathrm{~s} & 88009.29(3) & 156757.56(3) & \mathrm{pc}\end{array}$ $\begin{array}{lllllll}0.003 & 13 & 23 & 5 \mathrm{~d}^{5} 6 \mathrm{~s} & 54464.11(3) & 123202.06(3) & \mathrm{pc}\end{array}$ $\begin{array}{lllllll}-0.009 & 9 & 18 & 5 d^{5} 6 s & 80577.63(6) & 149313.18(6) & \text { pc }\end{array}$ $\begin{array}{llllllll}-0.001 & 19 & 22 & 5 d^{5} 6 s & 76295.33(5) & 145003.78(4) & \text { pc }\end{array}$ $\begin{array}{lllllll}0.001 & 14 & 16 & 5 \mathrm{~d}^{5} 6 \mathrm{~s} & 84734.80(4) & 153412.13(4) & \mathrm{pc}\end{array}$ $\begin{array}{lllllll}-0.001 & 8 & 21 & 5 d^{5} 6 \mathrm{~s} & 59206.99(2) & 127824.31(1) & \text { pc }\end{array}$ $\begin{array}{lllllll}-0.006 & 4 & 10 & 5 d^{5} 6 s & 31975.85(3) & 100573.47(3) & \text { pc }\end{array}$ $\begin{array}{lllllll}0.009 & 16 & 26 & 5 d^{5} 6 s & 71191.62(4) & 139739.81(5) & \text { pc }\end{array}$ $\begin{array}{lllllll}0.002 & 11 & 23 & 5 \mathrm{~d}^{5} 6 \mathrm{~s} & 69158.48(5) & 137685.88(5) & \mathrm{pc}\end{array}$ $\begin{array}{lllllll}-0.019 & 10 & 16 & 5 \mathrm{~d}^{6} & 52259.88(3) & 120722.59(4) & \text { pc } * 150, \text { Ir V }\end{array}$ $\begin{array}{lllllll}-0.003 & 7 & 14 & 5 d^{5} 6 s & 50417.92(2) & 118786.27(1) & \text { pc }\end{array}$ $\begin{array}{lllllll}-0.006 & 15 & 20 & 5 d^{5} 6 s & 76493.69(3) & 144855.15(3) & \text { pc }\end{array}$ $\begin{array}{llllllll}0.003 & 13 & 19 & 5 \mathrm{~d}^{5} 6 \mathrm{~s} & 74545.24(3) & 142865.10(2) & \text { pc } & \text { Ir III }\end{array}$ $\begin{array}{lllllll}-0.001 & 8 & 18 & 5 \mathrm{~d}^{5} 6 \mathrm{~s} & 70298.76(2) & 138612.56(2) & \mathrm{pc}\end{array}$ $\begin{array}{lllllll}-0.002 & 9 & 23 & 5 d^{5} 6 s & 54464.11(3) & 122746.29(2) & \text { pc }\end{array}$ $\begin{array}{lllllll}0.006 & 8 & 16 & 5 \mathrm{~d}^{5} 6 \mathrm{~s} & 84734.80(4) & 152982.27(4) & \text { pc }\end{array}$ $\begin{array}{lllllll}-0.006 & 13 & 21 & 5 \mathrm{~d}^{5} 6 \mathrm{~s} & 74057.35(4) & 142198.78(4) & \mathrm{pc}\end{array}$ $\begin{array}{lllllll}0.000 & 9 & 20 & 5 \mathrm{~d}^{5} 6 \mathrm{~s} & 76493.69(3) & 144626.15(2) & \mathrm{pc}\end{array}$ $\begin{array}{lllllll}0.003 & 9 & 23 & 5 \mathrm{~d}^{5} 6 \mathrm{~s} & 69158.48(5) & 137277.36(4) & \mathrm{pc}\end{array}$ $\begin{array}{lllllll}-0.001 & 9 & 14 & 5 \mathrm{~d}^{5} 6 \mathrm{~s} & 50417.92(2) & 118527.90(2) & \mathrm{pc}\end{array}$ $\begin{array}{lllllll}0.003 & 15 & 19 & 5 d^{5} 6 s & 73067.88(5) & 141141.16(4) & \text { pc }\end{array}$ $\begin{array}{lllllll}0.009 & 12 & 21 & 5 \mathrm{~d}^{5} 6 \mathrm{~s} & 69741.38(3) & 137700.64(3) & \text { pc } * 300, \text { Ir III }\end{array}$ $\begin{array}{lllllll}0.002 & 11 & 22 & 5 \mathrm{~d}^{5} 6 \mathrm{~s} & 58041.98(4) & 125982.14(5) & \mathrm{pc}\end{array}$ 


\begin{tabular}{|c|c|c|c|c|c|c|}
\hline 137 & 991 & 1472.478 & 67912.74 & 1472.4823 & 0.0030 & \\
\hline 143 & 42 & 1474.588 & 67815.56 & 1474.5941 & 0.0040 & \\
\hline 59 & 19 & 1475.443 & 67776.26 & 1475.4315 & 0.0050 & -0.52 \\
\hline 105 & 72 & 1476.946 & 67707.27 & 1476.9413 & 0.0030 & -0.23 \\
\hline 40 & 88 & 1477.206 & 67695.38 & 1477.2116 & 0.0031 & \\
\hline 95 & 29 & 1477.304 & 67690.87 & 1477.3104 & 0.00 & \\
\hline 111 & 902 & 1479.849 & 67574.45 & 512 & 0023 & 0.09 \\
\hline 45 & 202 & 1480.355 & 51.38 & & & 0.04 \\
\hline 58 & 107 & 14 & 37.57 & & 30 & -0.11 \\
\hline 98 & 184 & 877 & 6.47 & 760 & 0.0030 & -0.05 \\
\hline 156 & 238 & & 0.07 & & & -0.04 \\
\hline 66 & 29 & 148 & 67302.63 & & 0.00 & 0.25 \\
\hline 59 & 75 & & & & & -0.12 \\
\hline 599 & 1748 & 148 & 67242.33 & & & 0.28 \\
\hline 52 & 136 & 148 & 9.12 & & & -0.11 \\
\hline 117 & 90 & & & & & -0.18 \\
\hline 492 & 1089 & $14 \varepsilon$ & 8.26 & & & 0.19 \\
\hline 51 & 79 & & & & & -0.30 \\
\hline 36 & 106 & 14 & 3.56 & & 050 & -0.40 \\
\hline 84 & 185 & & & & & \\
\hline 73 & 559 & & .61 & & & 0.02 \\
\hline 116 & 102 & 14 & & & & -0.01 \\
\hline 82 & 192 & & & & & 0.10 \\
\hline 40 & 40 & 145 & 7.17 & & & -0.50 \\
\hline 27 & 90 & & 0.32 & & & 0.02 \\
\hline 46 & 90 & 145 & 0.32 & & & -0.18 \\
\hline 103 & 1856 & & 2.19 & & & -1.72 \\
\hline 124 & 859 & & 72.20 & & & 0.17 \\
\hline 42 & 22 & 149 & 66700.74 & & & -0.16 \\
\hline 79 & 98 & 149 & 1.90 & 9.8817 & 0.0030 & -0.03 \\
\hline 20 & 22 & 149 & 9.43 & & & 0.31 \\
\hline 78 & 16 & 1500.146 & 0.16 & & & -0.30 \\
\hline 498 & 1864 & 150 & 2.57 & & & 0.12 \\
\hline 280 & 1048 & & 4.22 & & & -0.04 \\
\hline 9 & 24 & 1505.395 & 66427.74 & 1505.3986 & & 0.15 \\
\hline 38 & 23 & 1506.704 & 70.03 & & & 0.12 \\
\hline 367 & 912 & 1506.776 & 66366.85 & 1506.7754 & 0.0030 & -0.04 \\
\hline 65 & 176 & 1506.970 & 66358.30 & 1506.9739 & 0.0030 & 0.15 \\
\hline 145 & 1150 & 1508.878 & 66274.40 & & & -0.19 \\
\hline 18 & 42 & 1509.076 & 66265.72 & 1509.0845 & 0.0050 & 0.38 \\
\hline 102 & 1198 & & 66258.62 & & & 0.14 \\
\hline 151 & 83 & 1509.660 & 66240.08 & 1509.6598 & 0.0030 & -0.01 \\
\hline 14 & 33 & 1509.863 & 66231.16 & 1509.8570 & 0.0030 & -0.28 \\
\hline 195 & 231 & 1511.545 & 66157.49 & 1511.5435 & 0.0040 & -0.05 \\
\hline
\end{tabular}

\begin{tabular}{|c|c|c|c|c|c|c|c|}
\hline-0.005 & 13 & 14 & $5 d^{5} 6 s$ & $50417.92(2)$ & $118330.45(3)$ & $\mathrm{pc}$ & \\
\hline-0.006 & 10 & 17 & $5 d^{5} 6 s$ & $87782.64(4)$ & $155597.91(4)$ & $\mathrm{pc}$ & \\
\hline 0.011 & 11 & 14 & $5 d^{5} 6 s$ & $82458.31(3)$ & $150235.09(3)$ & $\mathrm{pc} *$ & \\
\hline 0.005 & 9 & 20 & $5 d^{5} 6 s$ & $81605.68(5)$ & $149313.18(6)$ & $\mathrm{pc}$ & \\
\hline-0.006 & 10 & 19 & $5 d^{5} 6 s$ & $68255.33(2)$ & $135950.44(1)$ & $\mathrm{pc}$ & \\
\hline-0.006 & 12 & 13 & $5 d^{5} 6 s$ & $90497.75(4)$ & $158188.33(4)$ & $\mathrm{pc}$ & \\
\hline-0.002 & 13 & 23 & $5 d^{5} 6 s$ & $55627.70(2)$ & $123202.06(3)$ & $\mathrm{pc}$ & \\
\hline-0.001 & 8 & 9 & $5 d^{5} 6 s$ & $60272.97(1)$ & $127824.31(1)$ & pc & \\
\hline 0.002 & 12 & 21 & $5 d^{5} 6 s$ & $69741.38(3)$ & $137229.06(2)$ & pc & \\
\hline 0.001 & 14 & 22 & $5 d^{5} 6 s$ & $76295.33(5)$ & $143731.85(5)$ & $\mathrm{pc}$ & \\
\hline 0.001 & 11 & 12 & $5 d^{5} 6 s$ & $84005.15(4)$ & $151395.26(3)$ & $\mathrm{pc}$ & \\
\hline-0.006 & 13 & 17 & $5 d^{5} 6 s$ & $87782.64(4)$ & $155085.02(3)$ & $\mathrm{pc} *$ & \\
\hline 0.003 & 12 & 23 & $5 d^{5} 6 s$ & $69158.48(5)$ & $136429.29(5)$ & $\mathrm{pc}$ & \\
\hline-0.006 & 11 & 18 & $5 d^{5} 6 s$ & $58740.09(6)$ & $125982.14(5)$ & $\mathrm{pc}$ & \\
\hline 0.002 & 6 & 19 & $5 d^{5} 6 s$ & $68255.33(2)$ & $135484.56(1)$ & $\mathrm{pc}$ & Ir III \\
\hline 0.004 & 11 & 13 & $5 d^{5} 6 s$ & $90123.22(5)$ & $157327.79(6)$ & $\mathrm{pc}$ & \\
\hline-0.004 & 10 & 11 & $5 d^{5} 6 s$ & $75494.15(7)$ & $142662.22(6)$ & $\mathrm{pc}$ & \\
\hline 0.007 & 15 & 19 & $5 d^{5} 6 s$ & $68255.33(2)$ & $135420.03(3)$ & $\mathrm{pc}$ & \\
\hline 0.009 & 13 & 9 & $5 d^{5} 6 s$ & $85249.67(3)$ & $152373.63(3)$ & pc * & \\
\hline-0.005 & 6 & 19 & $5 d^{5} 6 s$ & $79208.51(3)$ & $146330.41(2)$ & pc & \\
\hline 0.000 & 9 & 23 & $5 d^{5} 6 s$ & $55627.70(2)$ & $122746.29(2)$ & pc & \\
\hline 0.000 & 8 & 9 & $5 d^{5} 6 s$ & $81351.39(2)$ & $148358.78(3)$ & $\mathrm{pc}$ & \\
\hline-0.002 & 16 & 20 & $5 d^{5} 6 s$ & $72737.30(6)$ & $139739.81(5)$ & $\mathrm{pc}$ & \\
\hline 0.011 & 17 & 19 & $5 d^{5} 6 s$ & $79208.51(3)$ & $146186.18(3)$ & $\mathrm{pc}$ & \\
\hline 0.000 & 12 & 18 & $5 d^{5} 6 s$ & $70298.76(2)$ & $137229.06(2)$ & $\mathrm{pc}$ & \\
\hline 0.004 & 15 & 19 & $5 d^{5} 6 s$ & $73067.88(5)$ & 139998.38(4) & $\mathrm{pc}$ & \\
\hline 0.038 & 8 & 18 & $5 d^{5} 6 s$ & $70298.76(2)$ & $137142.67(1)$ & $\mathrm{pc} *$ & $400, \mathrm{~m}(\mathrm{Ir}$ III $)$ \\
\hline-0.004 & 11 & 22 & $5 d^{5} 6 s$ & $59210.11(5)$ & $125982.14(5)$ & $\mathrm{pc}$ & \\
\hline 0.004 & 11 & 12 & $5 d^{5} 6 s$ & $84005.15(4)$ & $150706.05(4)$ & $\mathrm{pc} *$ & \\
\hline 0.001 & 16 & 19 & $5 d^{5} 6 s$ & $73067.88(5)$ & $139739.81(5)$ & $\mathrm{pc}$ & \\
\hline-0.007 & 14 & 19 & $5 d^{5} 6 s$ & $74545.24(3)$ & $141214.36(3)$ & $\mathrm{pc} *$ & \\
\hline 0.007 & 11 & 16 & $5 d^{5} 6 s$ & $84734.80(4)$ & $151395.26(3)$ & $\mathrm{pc} *$ & \\
\hline-0.003 & 2 & 10 & $5 d^{5} 6 s$ & $31975.85(3)$ & $98528.30(2)$ & pc & \\
\hline 0.001 & 11 & 26 & $5 d^{5} 6 s$ & $71191.62(4)$ & $137685.88(5)$ & $\mathrm{pc}$ & \\
\hline-0.003 & 11 & 23 & $5 d^{5} 6 s$ & $54464.11(3)$ & $120891.70(2)$ & $\mathrm{pc} *$ & \\
\hline-0.003 & 13 & 17 & $5 d^{5} 6 s$ & $87782.64(4)$ & $154152.55(3)$ & $\mathrm{pc} *$ & \\
\hline 0.001 & 10 & 22 & $5 d^{5} 6 s$ & $76295.33(5)$ & $142662.22(6)$ & $\mathrm{pc}$ & \\
\hline-0.003 & 8 & 25 & $5 d^{5} 6 s$ & $66110.16(3)$ & $132468.31(2)$ & $\mathrm{pc}$ & \\
\hline 0.004 & 14 & 23 & $5 d^{5} 6 s$ & $55627.70(2)$ & $121902.29(3)$ & $\mathrm{pc}$ & \\
\hline-0.009 & 10 & 16 & $5 d^{5} 6 s$ & $84734.80(4)$ & $151000.14(5)$ & $\mathrm{pc} *$ & \\
\hline-0.003 & 10 & 23 & $5 d^{5} 6 s$ & $54464.11(3)$ & $120722.59(4)$ & $\mathrm{pc}$ & \\
\hline 0.000 & 12 & 14 & $5 d^{5} 6 s$ & $91240.96(6)$ & $157481.05(5)$ & $\mathrm{pc}$ & \\
\hline 0.006 & 16 & 19 & $5 d^{5} 6 s$ & $79208.51(3)$ & $145439.95(4)$ & $\mathrm{pc} *$ & \\
\hline 0.001 & 11 & 20 & $5 d^{5} 6 s$ & $81605.68(5)$ & $147763.22(4)$ & $\mathrm{pc}$ & \\
\hline
\end{tabular}




\begin{tabular}{|c|c|c|c|c|c|c|c|c|c|c|c|c|c|}
\hline 49 & 43 & 1511.701 & 66150.63 & 1511.7035 & 0.0040 & 0.09 & -0.002 & 9 & 19 & $5 d^{5} 6 s$ & $79208.51(3)$ & $145359.05(2)$ & $\mathrm{pc}$ \\
\hline 42 & 23 & 1512.572 & 66112.56 & 1512.5790 & 0.0060 & 0.31 & -0.007 & 11 & 6 & $5 d^{5} 6 s$ & $67091.85(1)$ & $133204.10(2)$ & $\mathrm{pc} *$ \\
\hline 150 & 369 & 1513.155 & 66087.07 & 1513.1608 & 0.0040 & 0.24 & -0.005 & 11 & 14 & $5 d^{5} 6 s$ & $91240.96(6)$ & $157327.79(6)$ & $\mathrm{pc}$ \\
\hline 10 & 28 & 1513.520 & 66071.15 & 1513.5332 & 0.0032 & 0.57 & -0.013 & 13 & 16 & $5 d^{6}$ & $52259.88(3)$ & $118330.45(3)$ & $\mathrm{pc} *$ \\
\hline 77 & 51 & 1514.112 & 66045.31 & 1514.0999 & 0.0041 & -0.53 & 0.012 & 12 & 17 & $5 d^{5} 6 s$ & $87782.64(4)$ & $153828.48(4)$ & $\mathrm{pc}$ \\
\hline 408 & 1598 & 1514.823 & 66014.31 & 1514.8141 & 0.0022 & -0.39 & 0.009 & 15 & 22 & $5 d^{5} 6 s$ & $59210.11(5)$ & $125224.81(4)$ & pc bl(Ir III) \\
\hline 28 & 112 & 1515.338 & 65991.87 & 1515.3315 & 0.0024 & -0.29 & 0.007 & 15 & 24 & $5 d^{5} 6 s$ & $59232.65(3)$ & $125224.81(4)$ & $\mathrm{pc}$ \\
\hline 42 & 65 & 1515.561 & 65982.15 & 1515.5722 & 0.0030 & 0.47 & -0.011 & 15 & 19 & $5 d^{5} 6 s$ & $74545.24(3)$ & $140526.92(3)$ & $\mathrm{pc}$ \\
\hline 112 & 1642 & 1515.628 & 65979.24 & 1515.6563 & 0.0030 & 1.22 & -0.028 & 15 & 25 & $5 d^{5} 6 s$ & $66110.16(3)$ & $132088.18(4)$ & $\mathrm{pc} * 900, \mathrm{bl}(\mathrm{Ir}$ III $)$ \\
\hline 44 & 403 & 1516.810 & 65927.84 & 1516.8094 & 0.0040 & -0.02 & 0.001 & 7 & 7 & $5 d^{6}$ & $52858.41(2)$ & $118786.27(1)$ & pc \\
\hline 115 & 277 & 1517.986 & 65876.75 & 1517.9957 & 0.0040 & 0.41 & -0.009 & 12 & 17 & $5 d^{5} 6 s$ & $87782.64(4)$ & $153658.98(5)$ & $\mathrm{pc}$ \\
\hline 10 & 41 & 1521.327 & 65732.07 & 1521.3274 & 0.0030 & 0.00 & 0.000 & 8 & 23 & $5 d^{5} 6 s$ & $55627.70(2)$ & $121359.77(1)$ & $\mathrm{pc}$ \\
\hline 130 & 1318 & 1521.443 & 65727.09 & 1521.4549 & 0.0031 & 0.53 & -0.012 & 5 & 20 & $5 d^{5} 6 s$ & $72737.30(6)$ & $138463.86(7)$ & $\mathrm{pc}$ \\
\hline 35 & 23 & 1522.583 & 65677.88 & 1522.5940 & 0.0054 & 0.49 & -0.011 & 8 & 14 & $5 d^{5} 6 s$ & $82458.31(3)$ & $148135.70(2)$ & $\mathrm{pc} *$ \\
\hline 51 & 806 & 1522.753 & 65670.54 & 1522.7772 & 0.0042 & 1.06 & -0.024 & 9 & 7 & $5 d^{6}$ & $52858.41(2)$ & $118527.90(2)$ & $\mathrm{pc} * 400, \mathrm{bl}$ \\
\hline 25 & 88 & 1526.373 & 65514.79 & 1526.3764 & 0.0040 & 0.15 & -0.003 & 8 & 15 & $5 d^{5} 6 s$ & $75164.22(2)$ & $140678.86(1)$ & $\mathrm{pc}$ \\
\hline 281 & 329 & 1527.305 & 65474.79 & 1527.3077 & 0.0041 & 0.10 & -0.002 & 10 & 13 & $5 d^{5} 6 s$ & $90123.22(5)$ & $155597.91(4)$ & $\mathrm{pc}$ \\
\hline 13 & 56 & 1527.358 & 65472.52 & 1527.3695 & 0.0030 & 0.48 & -0.011 & 13 & 7 & $5 d^{6}$ & $52858.41(2)$ & $118330.45(3)$ & $\mathrm{pc}$ \\
\hline 30 & 32 & 1528.982 & 65402.98 & 1528.9856 & 0.0040 & 0.14 & -0.003 & 14 & 10 & $5 d^{5} 6 s$ & $88009.29(3)$ & $153412.13(4)$ & $\mathrm{pc} *$ \\
\hline 42 & 48 & 1529.424 & 65384.11 & 1529.4283 & 0.0030 & 0.20 & -0.005 & 10 & 20 & $5 d^{5} 6 s$ & $72737.30(6)$ & $138121.21(6)$ & $\mathrm{pc}$ \\
\hline 132 & 757 & 1529.918 & 65362.99 & 1529.9246 & 0.0031 & 0.29 & -0.007 & 15 & 15 & $5 d^{5} 6 s$ & $75164.22(2)$ & $140526.92(3)$ & Ir III (100) \\
\hline 24 & 199 & 1532.233 & 65264.22 & 1532.2383 & 0.0030 & 0.22 & -0.005 & 11 & 23 & $5 d^{5} 6 s$ & $55627.70(2)$ & $120891.70(2)$ & $\mathrm{pc}$ \\
\hline 109 & 1352 & 1533.309 & 65218.44 & 1533.3127 & 0.0031 & 0.17 & -0.004 & 8 & 14 & $5 d^{5} 6 s$ & $50417.92(2)$ & $115636.19(3)$ & $\mathrm{pc}$ \\
\hline 394 & 1761 & 1534.747 & 65157.34 & 1534.7465 & 0.0030 & 0.00 & 0.000 & 10 & 20 & $5 d^{5} 6 s$ & $63912.64(4)$ & $129069.98(3)$ & $\mathrm{pc}$ \\
\hline 55 & 1118 & 1535.594 & 65121.40 & 1535.5966 & 0.0040 & 0.13 & -0.003 & 15 & 18 & $5 d^{5} 6 s$ & $70298.76(2)$ & $135420.03(3)$ & $\mathrm{pc} * 100, \mathrm{~m}$ \\
\hline 86 & 219 & 1537.194 & 65053.60 & 1537.2004 & 0.0030 & 0.27 & -0.006 & 10 & 19 & $5 d^{5} 6 s$ & $73067.88(5)$ & $138121.21(6)$ & pc \\
\hline 72 & 1162 & 1538.209 & 65010.68 & 1538.2142 & 0.0030 & 0.23 & -0.005 & 14 & 19 & $5 d^{5} 6 s$ & $79502.00(4)$ & $144512.45(5)$ & $\mathrm{pc}$ \\
\hline 70 & 318 & 1539.671 & 64948.96 & 1539.6750 & 0.0040 & 0.19 & -0.004 & 11 & 19 & $5 d^{5} 6 s$ & $68255.33(2)$ & $133204.10(2)$ & $\mathrm{pc}$ \\
\hline 75 & 106 & 1541.212 & 64883.99 & 1541.2255 & 0.0050 & 0.56 & -0.013 & 10 & 14 & $5 d^{5} 6 s$ & $82458.31(3)$ & $147341.74(3)$ & $\mathrm{pc} *$ \\
\hline 197 & 774 & 1542.110 & 64846.22 & 1542.1192 & 0.0030 & 0.39 & -0.009 & 15 & 22 & $5 d^{5} 6 s$ & $76295.33(5)$ & $141141.16(4)$ & $\mathrm{pc}$ \\
\hline 60 & 196 & 1543.625 & 64782.58 & 1543.6268 & 0.0030 & 0.08 & -0.002 & 14 & 19 & $5 d^{5} 6 s$ & $68255.33(2)$ & $133037.83(3)$ & $\mathrm{pc}$ \\
\hline 150 & 877 & 1544.041 & 64765.14 & 1544.0419 & 0.0032 & 0.06 & -0.001 & 10 & 25 & $5 d^{5} 6 s$ & $66110.16(3)$ & $130875.24(2)$ & pc \\
\hline 406 & 1400 & 1544.312 & 64753.74 & 1544.3240 & 0.0030 & 0.49 & -0.012 & 8 & 23 & $5 d^{5} 6 s$ & $69158.48(5)$ & $133911.73(6)$ & $\mathrm{pc}$ \\
\hline 241 & 1117 & 1548.449 & 64580.77 & 1548.4534 & 0.0031 & 0.20 & -0.005 & 14 & 20 & $5 d^{5} 6 s$ & $63912.64(4)$ & $128493.21(4)$ & pd \\
\hline 145 & 1893 & 1550.759 & 64484.57 & 1550.6896 & 0.0040 & -2.87 & 0.069 & 5 & 20 & $5 d^{5} 6 s$ & $72737.30(6)$ & $137224.74(7)$ & $\mathrm{pd} * 1000, \mathrm{~m}(\mathrm{C}$ IV $)$ \\
\hline 102 & 426 & 1552.589 & 64408.53 & 1552.5809 & 0.0041 & -0.35 & 0.008 & 13 & 18 & $5 d^{5} 6 s$ & $70298.76(2)$ & $134707.64(2)$ & $\mathrm{pd}$ \\
\hline 33 & 155 & 1552.710 & 64403.52 & 1552.7029 & 0.0030 & -0.30 & 0.007 & 17 & 26 & $5 d^{5} 6 s$ & $71191.62(4)$ & $135595.44(4)$ & $\mathrm{pd}$ \\
\hline 113 & 1807 & 1555.290 & 64296.69 & 1555.2905 & 0.0030 & 0.02 & -0.001 & 7 & 22 & $5 d^{5} 6 s$ & $58041.98(4)$ & $122338.65(5)$ & pd \\
\hline 70 & 91 & 1560.889 & 64066.07 & 1560.8954 & 0.0040 & 0.28 & -0.007 & 10 & 6 & $5 d^{5} 6 s$ & $71884.65(1)$ & $135950.44(1)$ & pe \\
\hline 14 & 37 & 1560.936 & 64064.12 & 1560.9442 & 0.0030 & 0.33 & -0.008 & 9 & 23 & $5 d^{5} 6 s$ & $54464.11(3)$ & $118527.90(2)$ & pe \\
\hline 36 & 35 & 1561.683 & 64033.50 & 1561.6891 & 0.0030 & 0.27 & -0.007 & 15 & 20 & $5 d^{5} 6 s$ & $76493.69(3)$ & $140526.92(3)$ & pe \\
\hline 141 & 1727 & 1562.612 & 63995.40 & 1562.6204 & 0.0030 & 0.33 & -0.008 & 13 & 21 & $5 d^{5} 6 s$ & $59206.99(2)$ & $123202.06(3)$ & pe \\
\hline 152 & 1577 & 1563.246 & 63969.47 & 1563.2472 & 0.0030 & 0.07 & -0.002 & 13 & 24 & $5 d^{5} 6 s$ & $59232.65(3)$ & $123202.06(3)$ & pe \\
\hline 80 & 129 & 1563.764 & 63948.25 & 1563.7598 & 0.0030 & -0.19 & 0.005 & 16 & 19 & $5 d^{5} 6 s$ & $79208.51(3)$ & $143156.95(4)$ & pe \\
\hline
\end{tabular}


$\begin{array}{lll}338 & 1004 & 1564.471\end{array}$ $\begin{array}{lll}660 & 2302 & 1565.648\end{array}$ $\begin{array}{lll}47 & 203 & 1565.768\end{array}$ $\begin{array}{lll}181 & 1704 & 1565.919\end{array}$ $\begin{array}{lll}32 & 59 & 1566.540\end{array}$ $\begin{array}{lll}246 & 138 & 1567.704\end{array}$ $\begin{array}{lll}64 & 109 & 1570.057\end{array}$ $\begin{array}{lll}68 & 51 & 1570.977\end{array}$ $\begin{array}{lll}228 & 1027 & 1571.265\end{array}$ $\begin{array}{lll}137 & 1110 & 1571.564\end{array}$ $\begin{array}{lll}80 & 95 & 1571.627\end{array}$

$\begin{array}{lll}96 & 420 & 1572.317\end{array}$ $\begin{array}{lll}275 & 1958 & 1572.363\end{array}$ $80 \quad 46 \quad 1573.916$ $\begin{array}{lll}171 & 1942 & 1574.469\end{array}$ $\begin{array}{lll}36 & 92 & 1574.682\end{array}$

$\begin{array}{lll}40 & 57 & 1575.736\end{array}$

$\begin{array}{lll}101 & 318 & 1576.174\end{array}$

$\begin{array}{lll}192 & 1683 & 1577.265\end{array}$

$\begin{array}{lll}47 & 878 & 1577.552\end{array}$

$\begin{array}{lll}34 & 398 & 1577.868\end{array}$

$\begin{array}{lll}31 & 26 & 1578.263\end{array}$

$\begin{array}{lll}141 & 116 & 1578.872\end{array}$

$\begin{array}{lll}11 & 41 & 1583.314\end{array}$

$\begin{array}{lll}477 & 1828 & 1584.065\end{array}$

$\begin{array}{lll}176 & 187 & 1585.973\end{array}$

$\begin{array}{lll}308 & 1308 & 1588.065\end{array}$

$\begin{array}{lll}51 & 443 & 1589.076\end{array}$

$\begin{array}{lll}169 & 1850 & 1589.663\end{array}$

$\begin{array}{lll}47 & 374 & 1589.814\end{array}$

$\begin{array}{lll}62 & 48 & 1590.854\end{array}$

$\begin{array}{lll}47 & 113 & 1591.502\end{array}$

$\begin{array}{lll}33 & 558 & 1592.916\end{array}$

$\begin{array}{lll}70 & 216 & 1593.905\end{array}$

$\begin{array}{lll}36 & 40 & 1594.073\end{array}$

$\begin{array}{lll}62 & 519 & 1594.498\end{array}$

$\begin{array}{lll}104 & 1294 & 1594.820\end{array}$

$\begin{array}{lll}137 & 375 & 1594.975\end{array}$

$\begin{array}{lll}442 & 1962 & 1595.382\end{array}$

$\begin{array}{lll}64 & 558 & 1595.670\end{array}$

$\begin{array}{lll}303 & 1302 & 1596.752\end{array}$

$\begin{array}{lll}47 & 99 & 1596.938\end{array}$

$\begin{array}{lll}33 & 56 & 1598.837\end{array}$

$\begin{array}{lll}59 & 68 & 1599.067\end{array}$ $\begin{array}{lllll}63919.36 & 1564.4641 & 0.0040 & -0.29\end{array}$ $\begin{array}{llll}63871.33 & 1565.6528 & 0.0042 & 0.21\end{array}$ $\begin{array}{lllll}63866.41 & 1565.7700 & 0.0030 & 0.07\end{array}$ $\begin{array}{lllll}63860.25 & 1565.9179 & 0.0030 & -0.06\end{array}$ $\begin{array}{lllll}63834.96 & 1566.5566 & 0.0030 & 0.69\end{array}$ $\begin{array}{lllll}63787.55 & 1567.7033 & 0.0050 & -0.03\end{array}$ $\begin{array}{lllll}63691.96 & 1570.0561 & 0.0030 & -0.03\end{array}$ $\begin{array}{lllll}63654.64 & 1570.9734 & 0.0050 & -0.16\end{array}$ $\begin{array}{llll}63643.00 & 1571.2576 & 0.0040 & -0.29\end{array}$ $\begin{array}{lllll}63630.87 & 1571.5578 & 0.0030 & -0.26\end{array}$ $\begin{array}{lllll}63628.35 & 1571.6220 & 0.0032 & -0.18\end{array}$ $\begin{array}{lllll}63600.39 & 1572.3125 & 0.0040 & -0.20\end{array}$ $\begin{array}{lllll}63598.56 & 1572.3626 & 0.0040 & 0.00\end{array}$ $\begin{array}{llll}63535.81 & 1573.9168 & 0.0040 & 0.05\end{array}$ $\begin{array}{lllll}63513.47 & 1574.4649 & 0.0030 & -0.17\end{array}$ $\begin{array}{llll}63504.90 & 1574.6868 & 0.0031 & 0.21\end{array}$ $\begin{array}{lllll}63462.42 & 1575.7282 & 0.0040 & -0.30\end{array}$ $\begin{array}{lllll}63444.76 & 1576.1813 & 0.0030 & 0.28\end{array}$ $\begin{array}{lllll}63400.89 & 1577.2597 & 0.0030 & -0.21\end{array}$ $\begin{array}{lllll}63389.34 & 1577.5486 & 0.0030 & -0.15\end{array}$ $\begin{array}{llll}63376.64 & 1577.8767 & 0.0041 & 0.33\end{array}$ $\begin{array}{lllll}63360.80 & 1578.2478 & 0.0030 & -0.60\end{array}$ $\begin{array}{lllll}63336.35 & 1578.8662 & 0.0050 & -0.24\end{array}$ $\begin{array}{lllll}63158.65 & 1583.3164 & 0.0030 & 0.08\end{array}$ $\begin{array}{lllll}63128.72 & 1584.0696 & 0.0031 & 0.18\end{array}$ $\begin{array}{llll}63052.79 & 1585.9736 & 0.0040 & 0.04\end{array}$ $\begin{array}{lllll}62969.71 & 1588.0651 & 0.0040 & 0.00\end{array}$ $\begin{array}{lllll}62929.64 & 1589.0748 & 0.0030 & -0.06\end{array}$ $\begin{array}{lllll}62906.42 & 1589.6540 & 0.0032 & -0.35\end{array}$ $\begin{array}{llll}62900.46 & 1589.8201 & 0.0032 & 0.26\end{array}$ $\begin{array}{llll}62859.31 & 1590.8608 & 0.0040 & 0.26\end{array}$ $\begin{array}{lllll}62833.72 & 1591.5029 & 0.0030 & 0.03\end{array}$ $\begin{array}{llll}62777.93 & 1592.9203 & 0.0040 & 0.15\end{array}$ $\begin{array}{lllll}62739.01 & 1593.9031 & 0.0030 & -0.06\end{array}$ $\begin{array}{lllll}62732.39 & 1594.0797 & 0.0040 & 0.27\end{array}$ $\begin{array}{lllll}62715.66 & 1594.4991 & 0.0040 & 0.04\end{array}$ $\begin{array}{lllll}62703.02 & 1594.8264 & 0.0030 & 0.27\end{array}$ $\begin{array}{lllll}62696.92 & 1594.9782 & 0.0030 & 0.14\end{array}$ $\begin{array}{lllll}62680.93 & 1595.3897 & 0.0031 & 0.32\end{array}$ $\begin{array}{lllll}62669.60 & 1595.6690 & 0.0030 & -0.04\end{array}$ $\begin{array}{lllll}62627.12 & 1596.7539 & 0.0031 & 0.06\end{array}$ $\begin{array}{lllll}62619.83 & 1596.9362 & 0.0031 & -0.08\end{array}$ $\begin{array}{lllll}62545.46 & 1598.8368 & 0.0031 & -0.01\end{array}$ $\begin{array}{llll}62536.46 & 1599.0682 & 0.0040 & 0.04\end{array}$ $\begin{array}{rrrrr}0.007 & 12 & 14 & 5 d^{5} 6 s & 91240.96(6)\end{array}$ $-0.005$ $\begin{array}{lllll}-0.002 & 13 & 23 & 5 d^{5} 6 s & 54464.11(3)\end{array}$ $\begin{array}{lllll}0.001 & 14 & 22 & 5 d^{5} 6 s & 58041.98(4)\end{array}$ $\begin{array}{lllll}-0.017 & 16 & 20 & 5 d^{5} 6 s & 81605.68(5)\end{array}$ $\begin{array}{lllll}0.001 & 4 & 9 & 5 d^{5} 6 s & 95628.69(5)\end{array}$ $\begin{array}{lllll}0.001 & 12 & 20 & 5 d^{5} 6 s & 72737.30(6)\end{array}$ $\begin{array}{lllll}0.004 & 13 & 13 & 5 d^{5} 6 s & 90497.75(4)\end{array}$ $\begin{array}{lllll}0.007 & 12 & 21 & 5 d^{5} 6 s & 74057.35(4)\end{array}$ $\begin{array}{lllll}0.006 & 10 & 25 & 5 d^{5} 6 s & 66110.16(3)\end{array}$ $\begin{array}{lllll}0.004 & 11 & 21 & 5 d^{5} 6 s & 74057.35(4)\end{array}$ $\begin{array}{llllll}0.005 & 5 & 11 & 5 \mathrm{~d}^{5} 6 \mathrm{~s} & 75494.15(7)\end{array}$ $\begin{array}{lllll}0.000 & 7 & 18 & 5 d^{5} 6 s & 58740.09(6)\end{array}$ $\begin{array}{lllll}-0.001 & 12 & 13 & 5 d^{5} 6 s & 90123.22(5)\end{array}$ $\begin{array}{lllll}0.004 & 9 & 24 & 5 d^{5} 6 s & 59232.65(3)\end{array}$ $\begin{array}{lllll}-0.005 & 15 & 20 & 5 d^{5} 6 s & 76493.69(3)\end{array}$ $\begin{array}{lllll}0.007 & 11 & 21 & 5 d^{5} 6 s & 69741.38(3)\end{array}$ $\begin{array}{lllll}-0.007 & 16 & 22 & 5 d^{5} 6 s & 76295.33(5)\end{array}$ $\begin{array}{lllll}0.005 & 6 & 22 & 5 d^{5} 6 s & 59210.11(5)\end{array}$ $\begin{array}{lllll}0.004 & 8 & 14 & 5 d^{5} 6 s & 50417.92(2)\end{array}$ $\begin{array}{lllll}-0.008 & 8 & 16 & 5 d^{6} & 52259.88(3)\end{array}$

0.015

0.006

$-0.002$

$-0.005$

$-0.001$

0.000

0.002

0.009

$-0.006$

$-0.007$

$-0.001$

$-0.004$

0.002

$-0.007$

$-0.001$

$-0.007$

$-0.004$

$-0.008$

0.001

$-0.001$

0.002

0.000
-0.001

$-0.001$
155160.61(5) pe Ir III (200)

122611.21(6) pe

$118330.45(3)$ pe

121902.29(3) pe

145439.95(4) pe *

159416.27(6) pe

136429.29(5) pe

$154152.55(3)$ pe

137700.64(3) pe

129741.29(3) pe

137685.88(5) pe

139094.74(6) pe

122338.65(5) pe

153658.98(5) pe

122746.29(2) pe

139998.38(4) pe

133204.10(2) pe

139739.81(5) pe

122611.21(6) pe

113807.41(2) pe

115636.19(3) pe

$136429.29(5)$ pe *

147341.74(3) pe

$118786.27(1)$ pe

122338.65(5) pe

154293.71(6) pe

138463.86(7) pe

132088.18(4) pe

$144512.45(5)$ pe Ir III (900)

118527.90(2) pe

152982.27(4) pe

134025.31(4) pe

115636.19(3) pe

133037.83(3) pe

137277.36(4) pe*

127824.31(1) pe

118330.45(3) pe

142198.78(4) pe

120722.59(4) pe

121902.29(3) pe

138121.21(6) pe

130875.24(2) pe

$145003.78(4)$ pe

137700.64(3) pe 


\begin{tabular}{|c|c|c|c|c|c|c|}
\hline 137 & 766 & 1599.293 & 62527.64 & 1599.2949 & & \\
\hline 87 & 75 & 1601.214 & 62452.63 & 1601.2182 & & \\
\hline 18 & 32 & 1603.007 & 62382.75 & 1602.9995 & 0.0030 & -0.30 \\
\hline 60 & 850 & 1603.924 & 52347.09 & 1603.9316 & 0031 & 0.29 \\
\hline 28 & 98 & 1607.162 & 2221.46 & 1607.1703 & 0033 & 0.30 \\
\hline 92 & 272 & 1608.201 & 2181.30 & & 0.00 & 0.07 \\
\hline 29 & 82 & 1608 & 2169.58 & 5045 & & 0.03 \\
\hline 120 & 1780 & 1608.934 & 2152.97 & 1608.9385 & & 0.19 \\
\hline 61 & 259 & 1609.623 & 2126.33 & 1609.6276 & & 0.16 \\
\hline 152 & 1123 & 1610 & 620 & 7057 & & 0.22 \\
\hline 21 & 177 & & .65 & & & -0.19 \\
\hline 17 & 81 & & 61978.69 & & 50 & 0.24 \\
\hline 56 & 330 & & & & & 0.35 \\
\hline 244 & 2074 & & 30.68 & & & 0.10 \\
\hline 94 & 768 & 70 & .07 & & & -0.03 \\
\hline 196 & 2019 & & & & & 0.13 \\
\hline 34 & 400 & & & & & -0.20 \\
\hline 39 & 212 & & & & & -0.12 \\
\hline 24 & 765 & 16 & .93 & 606 & & 0.40 \\
\hline 71 & 1432 & & & & & -0.18 \\
\hline 77 & 1258 & & .04 & & & 0.11 \\
\hline 40 & 930 & & & & & -0.42 \\
\hline 110 & 713 & & & & & 0.21 \\
\hline 124 & 1566 & & & & & -0.04 \\
\hline 377 & 1810 & & & 599 & & -0.11 \\
\hline 225 & 1815 & & 1.96 & & & -0.12 \\
\hline 86 & 683 & & 3.56 & & & -0.30 \\
\hline 86 & 1360 & & & & & -0.02 \\
\hline 104 & 1321 & & & & & -0.03 \\
\hline 18 & 18 & & 4.77 & 748 & 0.0040 & -0.15 \\
\hline 16 & 162 & & 8.90 & & & -0.10 \\
\hline 59 & 135 & 1642.104 & 97.50 & & 0.0041 & -0.01 \\
\hline 79 & 413 & & & & & 0.08 \\
\hline 82 & 241 & & & & & -0.20 \\
\hline 139 & 1365 & & 60843.82 & & & -0.03 \\
\hline 30 & 98 & & 60790.03 & & & 0.16 \\
\hline 43 & 81 & 1645.114 & 60786.05 & 1645.1097 & 0.0041 & -0.17 \\
\hline 42 & 139 & 5.177 & 60783.74 & 45.1787 & & 0.07 \\
\hline 79 & 184 & & & & & 0.02 \\
\hline 302 & 1876 & 1648.116 & 60675.33 & 1648.1122 & 0.0032 & -0.15 \\
\hline 72 & 1298 & & & & & -0.10 \\
\hline 140 & 915 & 1650.355 & 60593.01 & 1650.3529 & 0.0030 & -0.09 \\
\hline 58 & 910 & & 60583.62 & 1650.6101 & 0.0040 & -0.04 \\
\hline 29 & 574 & 1650.820 & 60575.96 & 1650.8057 & 0.0042 & -0.52 \\
\hline
\end{tabular}

\begin{tabular}{|c|c|c|c|c|c|c|c|}
\hline-0.002 & 17 & 19 & $5 d^{5} 6 s$ & $73067.88(5)$ & $135595.44(4)$ & pe & \\
\hline-0.005 & 11 & 17 & $5 d^{5} 6 s$ & $87782.64(4)$ & $150235.09(3)$ & pe & \\
\hline 0.008 & 14 & 25 & $5 d^{5} 6 s$ & $66110.16(3)$ & $128493.21(4)$ & pe * & \\
\hline-0.008 & 15 & 21 & $5 d^{5} 6 s$ & $69741.38(3)$ & $132088.18(4)$ & pe & \\
\hline-0.008 & 8 & 26 & $5 d^{5} 6 s$ & $71191.62(4)$ & $133412.78(5)$ & pe & \\
\hline-0.002 & 15 & 13 & $5 d^{5} 6 s$ & $90123.22(5)$ & $152304.45(5)$ & pe & \\
\hline-0.001 & 8 & 18 & $5 d^{5} 6 s$ & $70298.76(2)$ & $132468.31(2)$ & pe & \\
\hline-0.005 & 8 & 21 & $5 d^{5} 6 s$ & $59206.99(2)$ & $121359.77(1)$ & pe & \\
\hline-0.004 & 14 & 20 & $5 d^{5} 6 s$ & $81605.68(5)$ & $143731.85(5)$ & pe & \\
\hline-0.006 & 10 & 18 & $5 d^{5} 6 s$ & $80577.63(6)$ & $142662.22(6)$ & pe & 200 \\
\hline 0.005 & 12 & 15 & $5 d^{5} 6 s$ & $75164.22(2)$ & $137229.06(2)$ & pe & \\
\hline-0.006 & 8 & 15 & $5 d^{5} 6 s$ & $75164.22(2)$ & $137142.67(1)$ & pe & \\
\hline-0.009 & 10 & 22 & $5 d^{5} 6 s$ & $76295.33(5)$ & $138121.21(6)$ & pe & \\
\hline-0.002 & 5 & 11 & $5 d^{5} 6 s$ & $75494.15(7)$ & $137224.74(7)$ & pe & \\
\hline 0.001 & 11 & 9 & $5 d^{5} 6 s$ & $95628.69(5)$ & $157327.79(6)$ & pe * & * 100 \\
\hline-0.003 & 11 & 21 & $5 d^{5} 6 s$ & $59206.99(2)$ & $120891.70(2)$ & pe & \\
\hline 0.005 & 11 & 24 & $5 d^{5} 6 s$ & $59232.65(3)$ & $120891.70(2)$ & pe & \\
\hline 0.003 & 16 & 20 & $5 d^{5} 6 s$ & $81605.68(5)$ & $143156.95(4)$ & pe * & \\
\hline-0.010 & 8 & 16 & $5 d^{6}$ & $52259.88(3)$ & $113807.41(2)$ & pe & \\
\hline 0.005 & 10 & 22 & $5 d^{5} 6 s$ & $59210.11(5)$ & $120722.59(4)$ & pe & \\
\hline-0.003 & 10 & 24 & $5 d^{5} 6 s$ & $59232.65(3)$ & $120722.59(4)$ & pe & \\
\hline 0.011 & 10 & 19 & $5 d^{5} 6 s$ & $68255.33(2)$ & $129741.29(3)$ & pe & \\
\hline-0.006 & 15 & 21 & $5 d^{5} 6 s$ & $74057.35(4)$ & $135420.03(3)$ & pe & \\
\hline 0.001 & 15 & 20 & $5 d^{5} 6 s$ & $63912.64(4)$ & $125224.81(4)$ & pe & \\
\hline 0.003 & 8 & 20 & $5 d^{5} 6 s$ & $72737.30(6)$ & $133911.73(6)$ & $\mathrm{pf}$ & \\
\hline 0.003 & 8 & 23 & $5 d^{5} 6 s$ & $54464.11(3)$ & $115636.19(3)$ & $\mathrm{pf}$ & \\
\hline 0.008 & 10 & 21 & $5 d^{5} 6 s$ & $69741.38(3)$ & $130875.24(2)$ & $\mathrm{pf}$ & \\
\hline 0.001 & 8 & 9 & $5 d^{5} 6 s$ & $60272.97(1)$ & $121359.77(1)$ & $\mathrm{pf}$ & \\
\hline 0.001 & 15 & 14 & $5 d^{5} 6 s$ & $91240.96(6)$ & $152304.45(5)$ & $\mathrm{pf}$ & 300 \\
\hline 0.004 & 15 & 19 & $5 d^{5} 6 s$ & $79502.00(4)$ & $140526.92(3)$ & $\mathrm{pf} *$ & \\
\hline 0.003 & 8 & 7 & $5 d^{6}$ & $52858.41(2)$ & $113807.41(2)$ & $\mathrm{pf}$ & \\
\hline 0.000 & 11 & 13 & $5 d^{5} 6 s$ & $90497.75(4)$ & $151395.26(3)$ & $\mathrm{pf}$ & \\
\hline-0.002 & 15 & 19 & $5 d^{5} 6 s$ & $74545.24(3)$ & $135420.03(3)$ & pf & \\
\hline 0.005 & 11 & 19 & $5 d^{5} 6 s$ & $79208.51(3)$ & $140054.55(3)$ & $\mathrm{pf}$ & \\
\hline 0.001 & 8 & 19 & $5 d^{5} 6 s$ & $73067.88(5)$ & $133911.73(6)$ & $\mathrm{pf}$ & \\
\hline-0.004 & 15 & 19 & $5 d^{5} 6 s$ & $79208.51(3)$ & 139998.38(4) & $\mathrm{pf}$ & \\
\hline 0.005 & 10 & 15 & $5 d^{5} 6 s$ & $75164.22(2)$ & $135950.44(1)$ & $\mathrm{pf}$ & \\
\hline-0.002 & 9 & 20 & $5 d^{5} 6 s$ & $76493.69(3)$ & $137277.36(4)$ & $\mathrm{pf}$ & \\
\hline 0.000 & 16 & 14 & $5 d^{5} 6 s$ & $82458.31(3)$ & $143156.95(4)$ & $\mathrm{pf}$ & \\
\hline 0.004 & 8 & 20 & $5 d^{5} 6 s$ & $72737.30(6)$ & $133412.78(5)$ & $\mathrm{pf}$ & \\
\hline 0.003 & 11 & 9 & $5 d^{5} 6 s$ & $60272.97(1)$ & $120891.70(2)$ & $\mathrm{pf}$ & \\
\hline 0.002 & 13 & 20 & $5 d^{5} 6 s$ & $81605.68(5)$ & $142198.78(4)$ & $\mathrm{pf}$ & \\
\hline 0.001 & 8 & 6 & $5 d^{5} 6 s$ & $71884.65(1)$ & $132468.31(2)$ & $\mathrm{pf}$ & \\
\hline 0.014 & 10 & 18 & $5 d^{5} 6 s$ & $70298.76(2)$ & $130875.24(2)$ & $\mathrm{pf} *$ & 200, Ir III (400) \\
\hline
\end{tabular}




$\begin{array}{rrr}232 & 1733 & 1657.145 \\ 88 & 1123 & 1658.699 \\ 15 & 19 & 1659.229 \\ 200 & 1086 & 1660.089 \\ 83 & 89 & 1660.906 \\ 82 & 595 & 1662.953 \\ 29 & 120 & 1663.237 \\ 34 & 214 & 1663.631 \\ 103 & 1406 & 1666.437 \\ 72 & 1576 & 1667.552 \\ 235 & 1154 & 1672.868 \\ 24 & 110 & 1673.911 \\ 11 & 101 & 1678.436 \\ 67 & 34 & 1679.775 \\ 91 & 606 & 1681.240 \\ 86 & 910 & 1682.298 \\ 22 & 40 & 1683.382 \\ 143 & 1679 & 1685.114 \\ 10 & 72 & 1685.751 \\ 36 & 62 & 1686.340 \\ 30 & 316 & 1686.642 \\ 168 & 818 & 1690.564 \\ 13 & 145 & 1691.379 \\ 52 & 949 & 1692.108 \\ 34 & 114 & 1694.331 \\ 18 & 60 & 1694.998 \\ 34 & 103 & 1695.182 \\ 35 & 57 & 1695.478 \\ 35 & 23 & 1700.259 \\ 41 & 170 & 1702.077 \\ 59 & 94 & 1703.483 \\ 298 & 565 & 1704.591 \\ 19 & 21 & 1704.777 \\ 26 & 106 & 1707.951 \\ 16 & 285 & 1709.014 \\ 19 & 54 & 1711.569 \\ 24 & 41 & 1711.812 \\ 67 & 1404 & 1718.815 \\ 48 & 131 & 1720.159 \\ 40 & 122 & 1721.993 \\ 27 & 115 & 1723.224 \\ 44 & 306 & 1723.532 \\ 49 & 101 & 1726.902 \\ 372 & 1325 & 1727.532 \\ & & \end{array}$

$\begin{array}{lllll}60344.75 & 1657.1410 & 0.0032 & -0.15\end{array}$ $\begin{array}{llll}1658.6920 & 0.0030 & -0.26\end{array}$ $\begin{array}{lllll}60268.96 & 1659.2283 & 0.0040 & -0.02\end{array}$ $\begin{array}{lllll}60237.72 & 1660.0869 & 0.0031 & -0.09\end{array}$ $\begin{array}{lllll}60208.09 & 1660.9005 & 0.0040 & -0.21\end{array}$ $\begin{array}{lllll}60133.99 & 1662.9538 & 0.0030 & 0.03\end{array}$ $\begin{array}{lllll}60123.71 & 1663.2343 & 0.0040 & -0.11\end{array}$ $\begin{array}{llll}60109.47 & 1663.6339 & 0.0040 & 0.09\end{array}$ $\begin{array}{lllll}60008.27 & 1666.4309 & 0.0031 & -0.22\end{array}$ $\begin{array}{lllll}59968.13 & 1667.5571 & 0.0030 & 0.17\end{array}$ $\begin{array}{lllll}59777.58 & 1672.8660 & 0.0040 & -0.07\end{array}$ $\begin{array}{lllll}59740.32 & 1673.9071 & 0.0041 & -0.16\end{array}$ $\begin{array}{lllll}59579.26 & 1678.4359 & 0.0030 & -0.02\end{array}$ $\begin{array}{lllll}59531.80 & 1679.7711 & 0.0051 & -0.12\end{array}$ $\begin{array}{lllll}59479.92 & 1681.2354 & 0.0031 & -0.15\end{array}$ $\begin{array}{lllll}59442.52 & 1682.2972 & 0.0031 & -0.01\end{array}$ $\begin{array}{lllll}59404.23 & 1683.3869 & 0.0040 & 0.18\end{array}$ $\begin{array}{lllll}59343.18 & 1685.1102 & 0.0030 & -0.12\end{array}$ $\begin{array}{lllll}59320.75 & 1685.7462 & 0.0030 & -0.16\end{array}$ $\begin{array}{lllll}59300.02 & 1686.3375 & 0.0030 & -0.09\end{array}$ $\begin{array}{lllll}59289.40 & 1686.6416 & 0.0030 & -0.02\end{array}$ $\begin{array}{llll}59151.86 & 1690.5656 & 0.0030 & 0.06\end{array}$ $\begin{array}{lllll}59123.36 & 1691.3760 & 0.0032 & -0.10\end{array}$ $\begin{array}{lllll}59097.88 & 1692.1104 & 0.0030 & 0.08\end{array}$ $\begin{array}{lllll}59020.35 & 1694.3324 & 0.0031 & 0.06\end{array}$ $\begin{array}{lllll}58997.12 & 1695.0000 & 0.0051 & 0.07\end{array}$ $\begin{array}{lllll}58990.71 & 1695.1856 & 0.0040 & 0.12\end{array}$ $\begin{array}{lllll}58980.40 & 1695.4762 & 0.0030 & -0.08\end{array}$ $\begin{array}{lllll}58814.57 & 1700.2610 & 0.0060 & 0.07\end{array}$ $\begin{array}{lllll}58751.75 & 1702.0746 & 0.0030 & -0.08\end{array}$ $\begin{array}{lllll}58703.26 & 1703.4858 & 0.0041 & 0.10\end{array}$ $\begin{array}{lllll}58665.11 & 1704.5933 & 0.0040 & 0.09\end{array}$ $\begin{array}{lllll}58658.71 & 1704.7723 & 0.0050 & -0.15\end{array}$ $\begin{array}{llll}58549.68 & 1707.9516 & 0.0030 & 0.01\end{array}$ $\begin{array}{lllll}58513.29 & 1709.0132 & 0.0040 & -0.01\end{array}$ $\begin{array}{lllll}58425.91 & 1711.5665 & 0.0040 & -0.10\end{array}$ $\begin{array}{lllll}58417.62 & 1711.8135 & 0.0030 & 0.04\end{array}$ $\begin{array}{lllll}58179.61 & 1718.8123 & 0.0030 & -0.10\end{array}$ $\begin{array}{lllll}58134.15 & 1720.1599 & 0.0030 & 0.02\end{array}$ $\begin{array}{lllll}58072.24 & 1721.9938 & 0.0041 & 0.02\end{array}$ $\begin{array}{lllll}58030.76 & 1723.2219 & 0.0030 & -0.07\end{array}$ $\begin{array}{lllll}58020.40 & 1723.5273 & 0.0041 & -0.15\end{array}$ $\begin{array}{lllll}57907.17 & 1726.8986 & 0.0040 & -0.11\end{array}$ $\begin{array}{lllll}57886.06 & 1727.5266 & 0.0040 & -0.17\end{array}$ $\begin{array}{lllllll}0.004 & 8 & 19 & 5 \mathrm{~d}^{5} 6 \mathrm{~s} & 73067.88(5) & 133412.78(5) & \mathrm{pf}\end{array}$ $\begin{array}{llllllll}0.007 & 13 & 22 & 5 \mathrm{~d}^{5} 6 \mathrm{~s} & 58041.98(4) & 118330.45(3) & \text { pf }\end{array}$

$\begin{array}{llllllll}0.001 & 19 & 16 & 5 \mathrm{~d}^{5} 6 \mathrm{~s} & 84734.80(4) & 145003.78(4) & \text { pf * }\end{array}$

$\begin{array}{llllllll}0.002 & 16 & 19 & 5 \mathrm{~d}^{5} 6 \mathrm{~s} & 79502.00(4) & 139739.81(5) & \mathrm{pf}\end{array}$

$\begin{array}{llllllll}0.006 & 11 & 13 & 5 \mathrm{~d}^{5} 6 \mathrm{~s} & 90497.75(4) & 150706.05(4) & \mathrm{pf}\end{array}$

$\begin{array}{lllllll}-0.001 & 12 & 22 & 5 \mathrm{~d}^{5} 6 \mathrm{~s} & 76295.33(5) & 136429.29(5) & \mathrm{pf}\end{array}$

$\begin{array}{llllllll}0.003 & 9 & 14 & 5 \mathrm{~d}^{5} 6 \mathrm{~s} & 91240.96(6) & 151364.78(6) & \text { pf }\end{array}$

$\begin{array}{lllllll}-0.002 & 9 & 9 & 5 \mathrm{~d}^{5} 6 \mathrm{~s} & 85249.67(3) & 145359.05(2) & \mathrm{pf}\end{array}$

$\begin{array}{lllllll}0.006 & 8 & 23 & 5 \mathrm{~d}^{5} 6 \mathrm{~s} & 55627.70(2) & 115636.19(3) & \text { pf }\end{array}$

$\begin{array}{llllllll}-0.005 & 16 & 21 & 5 \mathrm{~d}^{5} 6 \mathrm{~s} & 74057.35(4) & 134025.31(4) & \text { pf } & 400, \mathrm{bl}(\mathrm{O} \\ & \mathrm{lII})\end{array}$

$\begin{array}{llllllll}0.002 & 14 & 16 & 5 \mathrm{~d}^{5} 6 \mathrm{~s} & 84734.80(4) & 144512.45(5) & \mathrm{pf}\end{array}$

$\begin{array}{llllllll}0.004 & 13 & 14 & 5 \mathrm{~d}^{5} 6 \mathrm{~s} & 82458.31(3) & 142198.78(4) & \mathrm{pf}\end{array}$

$\begin{array}{lllllll}0.000 & 7 & 21 & 5 \mathrm{~d}^{5} 6 \mathrm{~s} & 59206.99(2) & 118786.27(1) & \text { pf }\end{array}$

$\begin{array}{llllllll}0.003 & 12 & 9 & 5 \mathrm{~d}^{5} 6 \mathrm{~s} & 95628.69(5) & 155160.61(5) & \mathrm{pf} *\end{array}$

$\begin{array}{llllllll}0.004 & 16 & 19 & 5 \mathrm{~d}^{5} 6 \mathrm{~s} & 74545.24(3) & 134025.31(4) & \mathrm{pf}\end{array}$

$\begin{array}{lllllll}0.000 & 10 & 18 & 5 \mathrm{~d}^{5} 6 \mathrm{~s} & 70298.76(2) & 129741.29(3) & \mathrm{pf}\end{array}$

$\begin{array}{lllllll}-0.005 & 8 & 19 & 5 \mathrm{~d}^{5} 6 \mathrm{~s} & 79208.51(3) & 138612.56(2) & \mathrm{pf}\end{array}$

$\begin{array}{lllllll}0.003 & 8 & 23 & 5 \mathrm{~d}^{5} 6 \mathrm{~s} & 54464.11(3) & 113807.41(2) & \mathrm{pf}\end{array}$

$\begin{array}{lllllll}0.004 & 9 & 21 & 5 \mathrm{~d}^{5} 6 \mathrm{~s} & 59206.99(2) & 118527.90(2) & \mathrm{pf}\end{array}$

$\begin{array}{lllllll}0.003 & 17 & 22 & 5 \mathrm{~d}^{5} 6 \mathrm{~s} & 76295.33(5) & 135595.44(4) & \text { pf }\end{array}$

$\begin{array}{lllllll}0.001 & 13 & 20 & 5 \mathrm{~d}^{5} 6 \mathrm{~s} & 63912.64(4) & 123202.06(3) & \mathrm{pf}\end{array}$

$\begin{array}{lllllll}-0.002 & 16 & 12 & 5 \mathrm{~d}^{5} 6 \mathrm{~s} & 84005.15(4) & 143156.95(4) & \text { pf }\end{array}$

$\begin{array}{lllllll}0.003 & 13 & 21 & 5 \mathrm{~d}^{5} 6 \mathrm{~s} & 59206.99(2) & 118330.45(3) & \mathrm{pf}\end{array}$

$\begin{array}{lllllll}-0.002 & 13 & 24 & 5 \mathrm{~d}^{5} 6 \mathrm{~s} & 59232.65(3) & 118330.45(3) & \mathrm{pf}\end{array}$

$\begin{array}{lllllll}-0.002 & 15 & 19 & 5 d^{5} 6 \mathrm{~s} & 73067.88(5) & 132088.18(4) & \mathrm{pf}\end{array}$

$\begin{array}{lllllll}-0.002 & 14 & 16 & 5 \mathrm{~d}^{5} 6 \mathrm{~s} & 84734.80(4) & 143731.85(5) & \text { pf * }\end{array}$

$\begin{array}{lllllll}-0.003 & 10 & 6 & 5 \mathrm{~d}^{5} 6 \mathrm{~s} & 71884.65(1) & 130875.24(2) & \mathrm{pf}\end{array}$

$\begin{array}{lllllll}0.002 & 14 & 21 & 5 \mathrm{~d}^{5} 6 \mathrm{~s} & 74057.35(4) & 133037.83(3) & \mathrm{pf}\end{array}$

$\begin{array}{lllllll}-0.002 & 12 & 13 & 5 \mathrm{~d}^{5} 6 \mathrm{~s} & 90123.22(5) & 148937.72(5) & \text { pf * }\end{array}$

$\begin{array}{llllllll}0.002 & 14 & 21 & 5 \mathrm{~d}^{5} 6 \mathrm{~s} & 69741.38(3) & 128493.21(4) & \mathrm{pf}\end{array}$

$\begin{array}{lllllll}-0.003 & 11 & 9 & 5 \mathrm{~d}^{5} 6 \mathrm{~s} & 81351.39(2) & 140054.55(3) & \mathrm{pf}\end{array}$

$\begin{array}{lllllll}-0.003 & 7 & 9 & 5 \mathrm{~d}^{5} 6 \mathrm{~s} & 95628.69(5) & 154293.71(6) & \mathrm{pf}\end{array}$

$\begin{array}{llllllll}0.004 & 11 & 19 & 5 \mathrm{~d}^{5} 6 \mathrm{~s} & 74545.24(3) & 133204.10(2) & \text { pf * }\end{array}$

$\begin{array}{lllllll}0.000 & 10 & 26 & 5 \mathrm{~d}^{5} 6 \mathrm{~s} & 71191.62(4) & 129741.29(3) & \mathrm{pf}\end{array}$

$\begin{array}{llllllll}0.000 & 7 & 9 & 5 \mathrm{~d}^{5} 6 \mathrm{~s} & 60272.97(1) & 118786.27(1) & \text { pf }\end{array}$

$\begin{array}{lllllll}0.003 & 7 & 20 & 5 \mathrm{~d}^{5} 6 \mathrm{~s} & 63912.64(4) & 122338.65(5) & \mathrm{pf}\end{array}$

$\begin{array}{llllllll}-0.001 & 8 & 11 & 5 d^{5} 6 s & 75494.15(7) & 133911.73(6) & \text { pf }\end{array}$

$\begin{array}{lllllll}0.003 & 8 & 23 & 5 \mathrm{~d}^{5} 6 \mathrm{~s} & 55627.70(2) & 113807.41(2) & \mathrm{pf}\end{array}$

$\begin{array}{lllllll}-0.001 & 16 & 20 & 5 d^{5} 6 s & 81605.68(5) & 139739.81(5) & \text { pf }\end{array}$

$\begin{array}{lllllll}-0.001 & 9 & 14 & 5 \mathrm{~d}^{5} 6 \mathrm{~s} & 91240.96(6) & 149313.18(6) & \mathrm{pf}\end{array}$

$\begin{array}{lllllll}0.002 & 15 & 21 & 5 \mathrm{~d}^{5} 6 \mathrm{~s} & 74057.35(4) & 132088.18(4) & \mathrm{pf}\end{array}$

$\begin{array}{llllllll}0.005 & 12 & 19 & 5 \mathrm{~d}^{5} 6 \mathrm{~s} & 79208.51(3) & 137229.06(2) & \mathrm{pf}\end{array}$

$\begin{array}{lllllll}0.003 & 16 & 9 & 5 \mathrm{~d}^{5} 6 \mathrm{~s} & 85249.67(3) & 143156.95(4) & \mathrm{pf}\end{array}$

$\begin{array}{lllllll}0.005 & 5 & 18 & 5 \mathrm{~d}^{5} 6 \mathrm{~s} & 80577.63(6) & 138463.86(7) & \text { pf }\end{array}$ 


$\begin{array}{rrr}20 & 37 & 1727.766 \\ 31 & 37 & 1731.260 \\ 23 & 122 & 1733.208 \\ 201 & 1317 & 1735.617 \\ 48 & 138 & 1737.823 \\ 72 & 276 & 1738.178 \\ 34 & 130 & 1741.228 \\ 35 & 76 & 1745.077 \\ 69 & 826 & 1745.155 \\ 52 & 85 & 1747.603 \\ 35 & 43 & 1748.914 \\ 47 & 386 & 1751.568 \\ 35 & 47 & 1752.161 \\ 120 & 1031 & 1759.835 \\ 158 & 477 & 1765.313 \\ 41 & 91 & 1766.258 \\ 10 & 29 & 1773.462 \\ 8 & 47 & 1777.743 \\ 20 & 43 & 1782.733 \\ 40 & 65 & 1783.160 \\ 34 & 158 & 1783.598 \\ 35 & 35 & 1784.141 \\ 17 & 63 & 1787.644 \\ 24 & 141 & 1792.367 \\ 18 & 47 & 1795.862 \\ 12 & 37 & 1802.340 \\ 96 & 314 & 1813.310 \\ 32 & 88 & 1819.940 \\ 31 & 173 & 1825.135 \\ 28 & 145 & 1825.429 \\ 45 & 438 & 1831.092 \\ 23 & 79 & 1835.164 \\ 20 & 124 & 1837.635 \\ 21 & 24 & 1845.374 \\ 46 & 217 & 1850.714 \\ 52 & 50 & 1851.345 \\ 8 & 51 & 1852.205 \\ 44 & 223 & 1853.637 \\ 83 & 300 & 1854.914 \\ 197 & 1562 & 1870.812 \\ 26 & 49 & 1878.018 \\ 8 & 166 & 1878.109 \\ 24 & 62 & 1886.618 \\ 37 & 52 & 1892.674 \\ & & \end{array}$

$\begin{array}{lllll}57878.20 & 1727.7615 & 0.0030 & -0.16\end{array}$ $\begin{array}{lllll}57761.39 & 1731.2582 & 0.0061 & -0.07\end{array}$ $\begin{array}{lllll}57696.50 & 1733.1996 & 0.0060 & -0.26\end{array}$ $\begin{array}{lllll}57616.39 & 1735.6169 & 0.0030 & -0.01\end{array}$ $\begin{array}{lllll}57543.27 & 1737.8133 & 0.0030 & -0.31\end{array}$ $\begin{array}{lllll}57531.51 & 1738.1746 & 0.0030 & -0.11\end{array}$ $\begin{array}{lllll}57430.75 & 1741.2302 & 0.0040 & 0.09\end{array}$ $\begin{array}{lllll}57304.06 & 1745.0761 & 0.0040 & -0.03\end{array}$ $\begin{array}{lllll}57301.51 & 1745.1523 & 0.0030 & -0.08\end{array}$ $\begin{array}{lllll}57221.23 & 1747.6059 & 0.0030 & 0.09\end{array}$ $\begin{array}{lllll}57178.33 & 1748.9115 & 0.0040 & -0.09\end{array}$ $\begin{array}{lllll}57091.72 & 1751.5620 & 0.0030 & -0.18\end{array}$ $\begin{array}{lllll}57072.38 & 1752.1570 & 0.0040 & -0.13\end{array}$ $\begin{array}{rrrrr}56823.51 & 1759.8303 & 0.0030 & -0.15\end{array}$ $\begin{array}{lllll}56647.19 & 1765.3151 & 0.0041 & 0.08\end{array}$ $\begin{array}{lllll}56616.87 & 1766.2583 & 0.0040 & 0.01\end{array}$ $\begin{array}{lllll}56386.90 & 1773.4606 & 0.0040 & -0.03\end{array}$ $\begin{array}{lllll}56251.08 & 1777.7436 & 0.0040 & 0.00\end{array}$ $\begin{array}{lllll}56093.63 & 1782.7397 & 0.0050 & 0.19\end{array}$ $\begin{array}{llll}56080.23 & 1783.1605 & 0.0040 & 0.03\end{array}$ $\begin{array}{lllll}56066.44 & 1783.6017 & 0.0030 & 0.11\end{array}$ $\begin{array}{lllll}56049.37 & 1784.1404 & 0.0040 & -0.03\end{array}$ $\begin{array}{lllll}55939.54 & 1787.6404 & 0.0040 & -0.12\end{array}$ $\begin{array}{lllll}55792.14 & 1792.3675 & 0.0030 & 0.01\end{array}$ $\begin{array}{lllll}55683.57 & 1795.8499 & 0.0040 & -0.37\end{array}$ $\begin{array}{lllll}55483.44 & 1802.3399 & 0.0030 & 0.01\end{array}$ $\begin{array}{lllll}55147.77 & 1813.3136 & 0.0040 & 0.11\end{array}$ $\begin{array}{lllll}54946.86 & 1819.9445 & 0.0030 & 0.13\end{array}$ $\begin{array}{lllll}54790.46 & 1825.1333 & 0.0030 & -0.06\end{array}$ $\begin{array}{rrrrr}54781.66 & 1825.4324 & 0.0031 & 0.12\end{array}$ $\begin{array}{lllll}54612.21 & 1831.0850 & 0.0032 & -0.21\end{array}$ $\begin{array}{lllll}54491.03 & 1835.1668 & 0.0031 & 0.07\end{array}$ $\begin{array}{lllll}54417.77 & 1837.6337 & 0.0030 & -0.04\end{array}$ $\begin{array}{llll}54189.54 & 1845.3763 & 0.0050 & 0.05\end{array}$ $\begin{array}{lllll}54033.20 & 1850.7143 & 0.0031 & 0.01\end{array}$ $\begin{array}{lllll}54014.78 & 1851.3478 & 0.0040 & 0.08\end{array}$ $\begin{array}{lllll}53989.69 & 1852.2031 & 0.0040 & -0.07\end{array}$ $\begin{array}{lllll}53947.98 & 1853.6379 & 0.0030 & 0.01\end{array}$ $\begin{array}{lllll}53910.85 & 1854.9166 & 0.0052 & 0.07\end{array}$ $\begin{array}{lllll}53452.74 & 1870.8118 & 0.0041 & 0.01\end{array}$ $\begin{array}{lllll}53247.64 & 1878.0189 & 0.0030 & 0.04\end{array}$ $\begin{array}{lllll}53245.06 & 1878.1163 & 0.0040 & 0.22\end{array}$ $\begin{array}{lllll}53004.90 & 1886.6177 & 0.0031 & -0.01\end{array}$ $\begin{array}{llll}52835.29 & 1892.6794 & 0.0040 & 0.14\end{array}$ $\begin{array}{lllll}0.005 & 10 & 26 & 5 \mathrm{~d}^{5} 6 \mathrm{~s} & 71191.62(4)\end{array}$ $\begin{array}{lllll}0.002 & 11 & 13 & 5 \mathrm{~d}^{5} 6 \mathrm{~s} & 90123.22(5)\end{array}$

$\begin{array}{llllll}0.008 & 12 & 14 & 5 \mathrm{~d}^{5} 6 \mathrm{~s} & 91240.96(6)\end{array}$

$\begin{array}{lllll}0.000 & 8 & 22 & 5 d^{5} 6 \mathrm{~s} & 76295.33(5)\end{array}$

$\begin{array}{llllll}0.009 & 10 & 18 & 5 \mathrm{~d}^{5} 6 \mathrm{~s} & 80577.63(6)\end{array}$

$\begin{array}{llllll}0.003 & 16 & 20 & 5 \mathrm{~d}^{5} 6 \mathrm{~s} & 76493.69(3)\end{array}$

$\begin{array}{llllll}-0.003 & 16 & 10 & 5 \mathrm{~d}^{5} 6 \mathrm{~s} & 88009.29(3)\end{array}$

$\begin{array}{lllll}0.001 & 8 & 15 & 5 \mathrm{~d}^{5} 6 \mathrm{~s} & 75164.22(2)\end{array}$

$\begin{array}{lllll}0.002 & 14 & 26 & 5 d^{5} 6 s & 71191.62(4)\end{array}$

$\begin{array}{lllll}-0.003 & 19 & 17 & 5 \mathrm{~d}^{5} 6 \mathrm{~s} & 87782.64(4)\end{array}$

$\begin{array}{lllll}0.003 & 10 & 9 & 5 d^{5} 6 \mathrm{~s} & 81351.39(2)\end{array}$

$\begin{array}{llllll}0.006 & 13 & 25 & 5 \mathrm{~d}^{5} 6 \mathrm{~s} & 66110.16(3)\end{array}$

$\begin{array}{lllll}0.004 & 15 & 17 & 5 \mathrm{~d}^{5} 6 \mathrm{~s} & 87782.64(4)\end{array}$

$\begin{array}{lllll}0.004 & 11 & 23 & 5 \mathrm{~d}^{5} 6 \mathrm{~s} & 69158.48(5)\end{array}$

$\begin{array}{lllll}-0.003 & 5 & 18 & 5 \mathrm{~d}^{5} 6 \mathrm{~s} & 80577.63(6)\end{array}$

$\begin{array}{llllll}0.000 & 9 & 10 & 5 \mathrm{~d}^{5} 6 \mathrm{~s} & 88009.29(3)\end{array}$

$\begin{array}{llllll}0.001 & 17 & 19 & 5 \mathrm{~d}^{5} 6 \mathrm{~s} & 79208.51(3)\end{array}$

$\begin{array}{lllll}0.000 & 8 & 5 & 5 \mathrm{~d}^{5} 6 \mathrm{~s} & 65108.69(0)\end{array}$

$\begin{array}{llllll}-0.006 & 17 & 19 & 5 \mathrm{~d}^{5} 6 \mathrm{~s} & 79502.00(4)\end{array}$

$\begin{array}{lllll}-0.001 & 11 & 20 & 5 d^{5} 6 s & 81605.68(5)\end{array}$

$\begin{array}{lllll}-0.004 & 15 & 23 & 5 \mathrm{~d}^{5} 6 \mathrm{~s} & 69158.48(5)\end{array}$

$\begin{array}{lllll}0.001 & 11 & 12 & 5 \mathrm{~d}^{5} 6 \mathrm{~s} & 84005.15(4)\end{array}$

$\begin{array}{lllll}0.004 & 8 & 6 & 5 \mathrm{~d}^{5} 6 \mathrm{~s} & 71884.65(1)\end{array}$

$\begin{array}{lllll}0.000 & 14 & 25 & 5 \mathrm{~d}^{5} 6 \mathrm{~s} & 66110.16(3)\end{array}$

$\begin{array}{llllll}0.012 & 10 & 21 & 5 \mathrm{~d}^{5} 6 \mathrm{~s} & 74057.35(4)\end{array}$

$\begin{array}{lllll}0.000 & 15 & 21 & 5 d^{5} 6 s & 69741.38(3)\end{array}$

$\begin{array}{lllll}-0.004 & 16 & 10 & 5 d^{5} 6 s & 88009.29(3)\end{array}$

$\begin{array}{lllll}-0.004 & 13 & 19 & 5 \mathrm{~d}^{5} 6 \mathrm{~s} & 68255.33(2)\end{array}$

$\begin{array}{lllll}0.002 & 11 & 26 & 5 \mathrm{~d}^{5} 6 \mathrm{~s} & 71191.62(4)\end{array}$

$\begin{array}{lllll}-0.004 & 11 & 25 & 5 d^{5} 6 s & 66110.16(3)\end{array}$

$\begin{array}{llllll}0.007 & 10 & 25 & 5 \mathrm{~d}^{5} 6 \mathrm{~s} & 66110.16(3)\end{array}$

$\begin{array}{lllll}-0.002 & 9 & 19 & 5 \mathrm{~d}^{5} 6 \mathrm{~s} & 68255.33(2)\end{array}$

$\begin{array}{llllll}0.001 & 13 & 20 & 5 \mathrm{~d}^{5} 6 \mathrm{~s} & 63912.64(4)\end{array}$

$\begin{array}{lllll}-0.002 & 13 & 10 & 5 \mathrm{~d}^{5} 6 \mathrm{~s} & 88009.29(3)\end{array}$

$\begin{array}{llllll}0.000 & 15 & 26 & 5 \mathrm{~d}^{5} 6 \mathrm{~s} & 71191.62(4)\end{array}$

$\begin{array}{lllll}-0.003 & 14 & 13 & 5 \mathrm{~d}^{5} 6 \mathrm{~s} & 90497.75(4)\end{array}$

$\begin{array}{lllll}0.002 & 17 & 20 & 5 \mathrm{~d}^{5} 6 \mathrm{~s} & 81605.68(5)\end{array}$

$\begin{array}{llllll}0.000 & 14 & 19 & 5 \mathrm{~d}^{5} 6 \mathrm{~s} & 74545.24(3)\end{array}$

$\begin{array}{lllll}-0.002 & 8 & 19 & 5 \mathrm{~d}^{5} 6 \mathrm{~s} & 79502.00(4)\end{array}$

$\begin{array}{lllll}0.000 & 6 & 23 & 5 \mathrm{~d}^{5} 6 \mathrm{~s} & 69158.48(5)\end{array}$

$\begin{array}{lllll}-0.001 & 10 & 20 & 5 \mathrm{~d}^{5} 6 \mathrm{~s} & 76493.69(3)\end{array}$

$\begin{array}{llllll}-0.008 & 11 & 20 & 5 d^{5} 6 s & 72737.30(6)\end{array}$

$\begin{array}{lllll}0.000 & 9 & 21 & 5 \mathrm{~d}^{5} 6 \mathrm{~s} & 69741.38(3)\end{array}$

$-0.005$
129069.98(3) pf

pf *

$148937.72(5) \quad$ pf *

133911.73(6) pf

138121.21(6) pf

134025.31(4) pf

$145439.95(4) \quad \mathrm{pf}$

132468.31(2) pf

$128493.21(4) \quad \mathrm{pf}$

145003.78(4) pf

138529.81(3) pf

23202.06(3) pf

144855.15(3) pf

125982.14(5) pf

137224.74(7) pf

$144626.15(2) \quad \mathrm{pf}$

135595.44(4) pf *

121359.77(1) pf

135595.44(4) pf*

137685.88(5) pf

125224.81(4) pf

$140054.55(3) \quad$ pf

127824.31(1) pf

121902.29(3) pf

129741.29(3) pf *

125224.81(4) pf

143156.95(4) pf

$123202.06(3) \quad \mathrm{pf}$

125982.14(5) pf

120891.70(2) pf

120722.59(4) pf

122746.29(2) pf

$118330.45(3) \quad p f$

$142198.78(4) \quad$ pf *

25224.81(4) pf

$144512.45(5) \quad p f$

135595.44(4) pf*

128493.21(4) pf

$133412.78(5) \quad$ pf *

122611.21(6) pf

129741.29(3) pf

125982.14(5) pf *

122746.29(2) pf

$133412.78(5) \quad$ pf 


\begin{tabular}{|c|c|c|c|c|c|c|c|c|c|c|c|c|c|}
\hline 19 & 27 & 1898.999 & 52659.31 & 1899.0034 & 0.0050 & 0.11 & -0.004 & 16 & 13 & $5 d^{5} 6 s$ & $90497.75(4)$ & $143156.95(4)$ & $\mathrm{pf} *$ \\
\hline 23 & 24 & 1901.636 & 52586.29 & 1901.6403 & 0.0050 & 0.11 & -0.004 & 15 & 19 & $5 d^{5} 6 s$ & $79502.00(4)$ & $132088.18(4)$ & $\mathrm{pf} *$ \\
\hline 30 & 50 & 1901.994 & 52576.40 & 1901.9980 & 0.0040 & 0.11 & -0.004 & 10 & 20 & $5 d^{5} 6 s$ & $76493.69(3)$ & $129069.98(3)$ & pf \\
\hline 7 & 32 & 1905.080 & 52491.23 & 1905.0925 & 0.0050 & 0.34 & -0.012 & 14 & 14 & $5 d^{5} 6 s$ & $91240.96(6)$ & $143731.85(5)$ & $\mathrm{pf} *$ \\
\hline 17 & 36 & 1917.156 & 52160.60 & 1917.1445 & 0.0040 & -0.31 & 0.012 & 14 & 21 & $5 d^{5} 6 s$ & $69741.38(3)$ & $121902.29(3)$ & $\mathrm{pf} *$ \\
\hline 21 & 38 & 1923.095 & 51999.52 & 1923.0947 & 0.0030 & 0.00 & 0.000 & 14 & 20 & $5 d^{5} 6 s$ & $76493.69(3)$ & $128493.21(4)$ & pf \\
\hline 29 & 686 & 1925.864 & 51924.75 & 1925.8624 & 0.0040 & -0.04 & 0.002 & 11 & 21 & $5 d^{5} 6 s$ & $74057.35(4)$ & $125982.14(5)$ & $\mathrm{pf} * 150$, Ir III \\
\hline 11 & 22 & 1928.539 & 51852.74 & 1928.5395 & 0.0070 & 0.02 & -0.001 & 11 & 9 & $5 d^{5} 6 s$ & $81351.39(2)$ & $133204.10(2)$ & $\mathrm{pf} *$ \\
\hline 9 & 25 & 1939.332 & 51564.15 & 1939.3334 & 0.0040 & 0.04 & -0.002 & 10 & 23 & $5 d^{5} 6 s$ & $69158.48(5)$ & $120722.59(4)$ & $\mathrm{pf} *$ \\
\hline 78 & 1282 & 1955.140 & 51147.24 & 1955.1477 & 0.0053 & 0.21 & -0.008 & 7 & 26 & $5 d^{5} 6 s$ & $71191.62(4)$ & $122338.65(5)$ & $\mathrm{pg} * 500, \mathrm{Ir}$ III \\
\hline 24 & 56 & 1961.498 & 50981.44 & 1961.5070 & 0.0041 & 0.23 & -0.009 & 10 & 21 & $5 d^{5} 6 s$ & $69741.38(3)$ & $120722.59(4)$ & $\mathrm{pg}$ \\
\hline 23 & 123 & 1971.971 & 50710.68 & 1971.9716 & 0.0040 & 0.01 & -0.001 & 14 & 26 & $5 d^{5} 6 s$ & $71191.62(4)$ & $121902.29(3)$ & pg \\
\hline 72 & 1071 & 1993.801 & 50155.45 & 1993.7973 & 0.0060 & -0.10 & 0.004 & 4 & 14 & $5 d^{5} 6 s$ & $50417.92(2)$ & $100573.47(3)$ & pg \\
\hline 11 & 26 & 1999.197 & 50020.09 & 1999.1939 & 0.0050 & -0.07 & 0.003 & 16 & 12 & $5 d^{5} 6 s$ & $84005.15(4)$ & $134025.31(4)$ & pg* \\
\hline 30 & 22 & $2028.373 a$ & 49284.74 & 2029.0273 & 0.0050 & 0.04 & -0.002 & 14 & 19 & $5 d^{5} 6 s$ & $79208.51(3)$ & $128493.21(4)$ & pg* \\
\hline 12 & 19 & $2043.102 \mathrm{a}$ & 48929.49 & 2043.7577 & 0.0042 & 0.01 & 0.000 & 15 & 22 & $5 d^{5} 6 s$ & $76295.33(5)$ & $125224.81(4)$ & $\mathrm{pg} *$ \\
\hline
\end{tabular}


Table 2

Experimental and calculated energy levels $\left(\mathrm{cm}^{-1}\right)$ in the $5 \mathrm{~d}^{6}$ and $5 \mathrm{~d}^{5} 6 \mathrm{~s}$ configurations of Ir IV.

\begin{tabular}{|c|c|c|c|c|c|c|c|c|c|c|}
\hline $\mathrm{E}_{\mathrm{obs}}$ & $\mathrm{E}_{\text {calc }}$ & $\Delta$ & $\mathrm{N}$ & $\mathrm{N}_{\mathrm{opt}}$ & rwmv $_{1}$ & rwmv $_{2}$ & Unc & Composition & & \\
\hline \multicolumn{11}{|l|}{$\mathrm{J}=0$} \\
\hline ( & 115713.99 & - & & & & & & $79 \% 2 \mid\left({ }^{2} \mathrm{P}\right){ }^{3} \mathrm{P}$ & $+12 \% 2 \mid\left({ }^{2} S\right){ }^{1} S$ & \\
\hline - & 98349.07 & - & & & & & & $52 \% 2 \mid\left({ }^{2} \mathrm{~S}\right){ }^{11} \mathrm{~S}$ & $+14 \% 2\left({ }^{2} \mathrm{P}\right){ }^{3} \mathrm{P}$ & $+\left.10 \% 1\right|^{1} \mathrm{~S} 2$ \\
\hline- & 83439.83 & - & & & & & & $\left.65 \% 1\right|^{1} \mathrm{~S} 1$ & $+18 \% 2\left({ }^{4} \mathrm{P}\right){ }^{3} \mathrm{P}$ & $+\quad 7 \% 1{ }^{1} \mathrm{~S} 2$ \\
\hline - & 77659.60 & - & & & & & & $62 \% 2\left({ }^{4} \mathrm{P}\right){ }^{3} \mathrm{P}$ & $+15 \% 2\left({ }^{2} \mathrm{~S}\right){ }^{1} \mathrm{~S}$ & $+10 \% 1{ }^{1} \mathrm{~S} 1$ \\
\hline 65108.69 & 65077.01 & 31.68 & 5 & 4 & 0.12 & 0.03 & 0.17 & $89 \% 2 \mid\left({ }^{4} \mathrm{D}\right){ }^{5} \mathrm{D}$ & $+\quad 7 \% 2\left({ }^{4} \mathrm{P}\right){ }^{3} \mathrm{P}$ & $+\quad 1 \% 1{ }^{5} \mathrm{D}$ \\
\hline- & 49834.79 & - & & & & & & $\left.46 \% 1\right|^{3} \mathrm{P} 1$ & $+\left.30 \% 1\right|^{1} \mathrm{~S} 2$ & $+13 \% 1{ }^{3} \mathrm{P} 2$ \\
\hline- & 33580.63 & - & & & & & & $72 \% 1{ }^{3} \mathrm{P} 2$ & $+\left.23 \% 1\right|^{3} \mathrm{P} 1$ & $+\quad 3 \% 1{ }^{1} \mathrm{~S} 1$ \\
\hline- & 24933.89 & - & & & & & & $48 \% 1{ }^{1} \mathrm{~S} 2$ & $+\left.17 \% 1\right|^{5} \mathrm{D}$ & $+\left.12 \% 1\right|^{1} \mathrm{~S} 1$ \\
\hline 8687.05 & 8702.47 & -15.42 & 7 & 7 & 0.29 & 0.24 & 0.40 & $\left.75 \% 1\right|^{5} \mathrm{D}$ & $+\left.14 \% 1\right|^{3} \mathrm{P} 1$ & $+\quad 4 \% 1{ }^{3} \mathrm{P} 2$ \\
\hline \multicolumn{11}{|l|}{$\mathrm{J}=1$} \\
\hline - & 124621.60 & - & & & & & & $47 \% 2 \mid\left({ }^{2} \mathrm{D} 1\right)^{3} \mathrm{D}$ & $+37 \% 2 \mid\left({ }^{2} \mathrm{P}\right){ }^{1} \mathrm{P}$ & $+\quad 9 \% 2\left({ }^{2} \mathrm{D} 3\right)^{3} \mathrm{D}$ \\
\hline- & 118068.44 & - & & & & & & $47 \% 2 \mid\left({ }^{2} \mathrm{P}\right){ }^{1} \mathrm{P}$ & $+22 \% 2\left({ }^{2} \mathrm{P}\right){ }^{3} \mathrm{P}$ & $+15 \% 2\left({ }^{2} \mathrm{D} 1\right)^{3} \mathrm{D}$ \\
\hline- & 112029.29 & - & & & & & & $65 \% 2 \mid\left({ }^{2} \mathrm{P}\right){ }^{3} \mathrm{P}$ & $+10 \% 2\left({ }^{2} \mathrm{D} 1\right)^{3} \mathrm{D}$ & $+\quad 9 \% 2\left({ }^{2} \mathrm{P}\right){ }^{1} \mathrm{P}$ \\
\hline- & 96068.40 & - & & & & & & $79 \% 2 \mid\left({ }^{2} \mathrm{D} 2\right)^{3} \mathrm{D}$ & $+\quad 8 \% 2\left({ }^{4} \mathrm{D}\right){ }^{3} \mathrm{D}$ & $+\quad 4 \% 2\left({ }^{2} \mathrm{D} 1\right)^{3} \mathrm{D}$ \\
\hline - & 89804.52 & - & & & & & & $71 \% 2 \mid\left({ }^{2} \mathrm{~S}\right){ }^{3} \mathrm{~S}$ & $+11 \% 2\left({ }^{4} \mathrm{P}\right){ }^{3} \mathrm{P}$ & $+\quad 5 \% 2\left({ }^{2} \mathrm{P}\right){ }^{3} \mathrm{P}$ \\
\hline - & 87142.22 & - & & & & & & $32 \% 2\left({ }^{2} \mathrm{D} 3\right)^{3} \mathrm{D}$ & $+19 \% 2\left({ }^{4} \mathrm{P}\right){ }^{3} \mathrm{P}$ & $+13 \% 2\left({ }^{4} \mathrm{D}\right){ }^{3} \mathrm{D}$ \\
\hline- & 80397.53 & - & & & & & & $34 \% 2 \mid\left({ }^{4} \mathrm{D}\right){ }^{3} \mathrm{D}$ & $+28 \% 2\left({ }^{2} \mathrm{D} 3\right)^{3} \mathrm{D}$ & $+13 \% 2\left({ }^{4} \mathrm{P}\right){ }^{3} \mathrm{P}$ \\
\hline - & 76682.15 & - & & & & & & $34 \% 2\left({ }^{4} \mathrm{P}\right){ }^{3} \mathrm{P}$ & $+29 \% 2\left({ }^{4} \mathrm{D}\right){ }^{3} \mathrm{D}$ & $+27 \% 2\left({ }^{4} \mathrm{~F}\right){ }^{5} \mathrm{~F}$ \\
\hline 71884.65 & 71872.27 & 12.38 & 6 & 5 & 0.09 & 0.07 & 0.14 & $35 \% 2 \mid\left({ }^{4} \mathrm{~F}\right){ }^{5} \mathrm{~F}$ & $+20 \% 2\left({ }^{4} \mathrm{D}\right){ }^{5} \mathrm{D}$ & $+16 \% 2\left({ }^{2} \mathrm{D} 3\right)^{3} \mathrm{D}$ \\
\hline 67091.85 & 67087.33 & 4.52 & 6 & 3 & 0.22 & 0.09 & 0.30 & $42 \% 2 \mid\left({ }^{4} \mathrm{P}\right){ }^{5} \mathrm{P}$ & $+28 \% 2\left({ }^{4} \mathrm{D}\right){ }^{5} \mathrm{D}$ & $+\quad 9 \% 2\left({ }^{4} \mathrm{~F}\right){ }^{5} \mathrm{~F}$ \\
\hline 60272.97 & 60254.64 & 18.34 & 9 & 8 & 0.09 & 0.04 & 0.12 & $46 \% 2 \mid\left({ }^{4} \mathrm{P}\right){ }^{5} \mathrm{P}$ & $+44 \% 22\left({ }^{4} \mathrm{D}\right){ }^{5} \mathrm{D}$ & $+\quad 3 \% 2\left({ }^{4} \mathrm{~F}\right){ }^{5} \mathrm{~F}$ \\
\hline 42519.60 & 42521.77 & -2.17 & 3 & 2 & 0.48 & 0.43 & 0.60 & $\left.77 \% 1\right|^{3} \mathrm{P} 1$ & $+13 \% 1{ }^{3} \mathrm{P} 2$ & $+\quad 5 \% 11^{5} \mathrm{D}$ \\
\hline 34466.53 & 34458.35 & 8.18 & 8 & 6 & 0.29 & 0.31 & 0.40 & $\left.62 \% 1\right|^{3} \mathrm{P} 2$ & $+\left.18 \% 1\right|^{3} \mathrm{D}$ & $+\left.12 \% 1\right|^{3} \mathrm{P} 1$ \\
\hline 26238.28 & 26242.10 & -3.82 & 17 & 15 & 0.19 & 0.18 & 0.30 & $\left.72 \% 1\right|^{3} \mathrm{D}$ & $+\left.16 \% 1\right|^{3} \mathrm{P} 2$ & $+\quad 7 \% 1{ }^{5} \mathrm{D}$ \\
\hline 8526.56 & 8529.39 & -2.83 & 14 & 13 & 0.21 & 0.14 & 0.24 & $\left.81 \% 1\right|^{5} \mathrm{D}$ & $+\left.\quad 9 \% 1\right|^{3} \mathrm{P} 1$ & $+\left.\quad 6 \% 1\right|^{3} \mathrm{P} 2$ \\
\hline \multicolumn{11}{|c|}{ 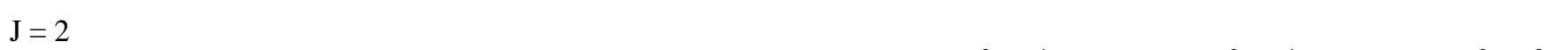 } \\
\hline- & 128459.24 & - & & & & & & $72 \% 2 \mid\left({ }^{2} \mathrm{D} 1\right)^{1} \mathrm{D}$ & $+14 \% 2 \mid\left({ }^{2} \mathrm{D} 3\right)^{1} \mathrm{D}$ & $+\quad 4 \% 2 \mid\left({ }^{2} \mathrm{P}\right){ }^{3} \mathrm{P}$ \\
\hline- & 122931.30 & - & & & & & & $62 \% 2 \mid\left({ }^{2} \mathrm{D} 1\right)^{3} \mathrm{D}$ & $+11 \% 2\left({ }^{2} \mathrm{D} 3\right)^{3} \mathrm{D}$ & $+\quad 7 \% 2\left({ }^{2} \mathrm{P}\right){ }^{3} \mathrm{P}$ \\
\hline- & 110681.51 & - & & & & & & $82 \% 2 \mid\left({ }^{2} \mathrm{P}\right){ }^{3} \mathrm{P}$ & $+\quad 4 \% 2\left({ }^{2} \mathrm{D} 2\right)^{1} \mathrm{D}$ & $+\quad 4 \% 2\left({ }^{2} \mathrm{D} 1\right)^{3} \mathrm{D}$ \\
\hline - & 105726.68 & - & & & & & & $58 \% 2 \mid\left({ }^{2} \mathrm{D} 2\right)^{1} \mathrm{D}$ & $+9 \% 2\left({ }^{2} \mathrm{D} 1\right)^{3} \mathrm{D}$ & $+\quad 8 \% 2\left({ }^{4} \mathrm{D}\right){ }^{3} \mathrm{D}$ \\
\hline- & 100939.94 & - & & & & & & $36 \% 2 \mid\left({ }^{2} \mathrm{D} 3\right)^{1} \mathrm{D}$ & $+20 \% 2\left({ }^{2} \mathrm{~F} 1\right)^{3} \mathrm{~F}$ & $+14 \% 2\left({ }^{4} \mathrm{P}\right){ }^{3} \mathrm{P}$ \\
\hline- & 97229.77 & - & & & & & & $75 \% 2 \mid\left({ }^{2} \mathrm{D} 2\right)^{3} \mathrm{D}$ & $+\quad 7 \% 2\left({ }^{2} \mathrm{~F} 2\right)^{3} \mathrm{~F}$ & $+\quad 5 \% 2\left({ }^{4} \mathrm{D}\right){ }^{5} \mathrm{D}$ \\
\hline 91225.56 & 91234.69 & -9.13 & 2 & 2 & 0.27 & 0.32 & 0.40 & $63 \% 2 \mid\left({ }^{4} \mathrm{~F}\right){ }^{3} \mathrm{~F}$ & $+19 \% 2\left({ }^{2} \mathrm{D} 3\right)^{3} \mathrm{D}$ & $+\quad 6 \% 2\left({ }^{2} \mathrm{~F} 1\right)^{3} \mathrm{~F}$ \\
\hline- & 88356.70 & - & & & & & & $27 \% 2 \mid\left({ }^{2} \mathrm{~F} 2\right)^{3} \mathrm{~F}$ & $+26 \% 2\left({ }^{4} \mathrm{P}\right){ }^{3} \mathrm{P}$ & $+17 \% 2\left({ }^{2} \mathrm{~F} 1\right)^{3} \mathrm{~F}$ \\
\hline- & 84774.17 & - & & & & & & $22 \% 2 \mid\left({ }^{2} \mathrm{D} 3\right)^{1} \mathrm{D}$ & $+17 \% 2\left({ }^{4} \mathrm{~F}\right){ }^{5} \mathrm{~F}$ & $+14 \% 2\left({ }^{2} \mathrm{D} 3\right)^{3} \mathrm{D}$ \\
\hline 81351.39 & 81363.81 & -12.42 & 9 & 7 & 0.09 & 0.07 & 0.14 & $44 \% 2 \mid\left({ }^{2} \mathrm{~F} 2\right)^{3} \mathrm{~F}$ & $\left.+12 \% 2{ }^{2} \mathrm{~F} 1\right)^{3} \mathrm{~F}$ & $+11 \% 2\left({ }^{4} \mathrm{P}\right){ }^{3} \mathrm{P}$ \\
\hline- & 77582.17 & - & & & & & & $43 \% 2\left({ }^{4} \mathrm{D}\right){ }^{3} \mathrm{D}$ & $+12 \% 2\left({ }^{2} \mathrm{D} 3\right)^{3} \mathrm{D}$ & $+11 \% 2\left({ }^{4} \mathrm{P}\right){ }^{5} \mathrm{P}$ \\
\hline 75164.22 & 75178.51 & -14.29 & 15 & 13 & 0.08 & 0.05 & 0.12 & $60 \% 2 \mid\left({ }^{4} \mathrm{~F}\right){ }^{5} \mathrm{~F}$ & $+\quad 7 \% 2\left({ }^{2} \mathrm{D} 3\right)^{1} \mathrm{D}$ & $+\quad 6 \% 2\left({ }^{4} \mathrm{D}\right){ }^{5} \mathrm{D}$ \\
\hline 70298.76 & 70319.04 & -20.28 & 18 & 12 & 0.09 & 0.07 & 0.12 & $21 \% 2 \mid\left({ }^{4} \mathrm{D}\right){ }^{5} \mathrm{D}$ & $+16 \% 2\left({ }^{2} \mathrm{D} 3\right)^{3} \mathrm{D}$ & $+10 \% 2\left({ }^{4} \mathrm{P}\right){ }^{5} \mathrm{P}$ \\
\hline 68255.33 & 68230.35 & 24.98 & 19 & 16 & 0.07 & 0.05 & 0.10 & $34 \% 2 \mid\left({ }^{4} \mathrm{P}\right){ }^{5} \mathrm{P}$ & $+14 \% 2 \mid\left({ }^{4} \mathrm{D}\right){ }^{3} \mathrm{D}$ & $+12 \% 2 \mid\left({ }^{4} \mathrm{D}\right){ }^{5} \mathrm{D}$ \\
\hline- & 63577.34 & - & & & & & & $\left.69 \% 1\right|^{1} \mathrm{D} 1$ & $+12 \% 1{ }^{1} \mathrm{D} 2$ & $+\quad 7 \% 2\left({ }^{2} \mathrm{D} 2\right)^{1} \mathrm{D}$ \\
\hline 59206.99 & 59203.27 & 3.72 & 21 & 18 & 0.07 & 0.07 & 0.10 & $41 \% 2 \mid\left({ }^{4} \mathrm{D}\right){ }^{5} \mathrm{D}$ & $+30 \% 2\left({ }^{4} \mathrm{P}\right){ }^{5} \mathrm{P}$ & $+10 \% 2\left({ }^{6} \mathrm{~S}\right){ }^{5} \mathrm{~S}$ \\
\hline 55627.70 & 55645.13 & -17.43 & 23 & 21 & 0.06 & 0.05 & 0.10 & $56 \% 2 \mid\left({ }^{4} \mathrm{G}\right){ }^{5} \mathrm{G}$ & $+15 \% 2\left({ }^{6} \mathrm{~S}\right){ }^{5} \mathrm{~S}$ & $+15 \% 2\left({ }^{2} \mathrm{~F} 1\right)^{3} \mathrm{~F}$ \\
\hline 52858.41 & 52873.93 & -15.52 & 7 & 5 & 0.12 & 0.13 & 0.16 & $38 \% 1{ }^{3} \mathrm{P} 1$ & $+\left.22 \% 1\right|^{3} \mathrm{P} 2$ & $+9 \% 2\left({ }^{6} \mathrm{~S}\right){ }^{5} \mathrm{~S}$ \\
\hline 50417.92 & 50399.27 & 18.65 & 14 & 12 & 0.09 & 0.05 & 0.12 & $50 \% 2 \mid\left({ }^{6} \mathrm{~S}\right){ }^{5} \mathrm{~S}$ & $+13 \% 2\left({ }^{4} \mathrm{P}\right){ }^{3} \mathrm{P}$ & $+\left.\quad 8 \% 1\right|^{3} \mathrm{P} 1$ \\
\hline 46564.59 & 46562.03 & 2.56 & 7 & 6 & 0.25 & 0.21 & 0.30 & $80 \% 1 \mid{ }^{3} \mathrm{~F} 1$ & $+\quad 6 \% 1{ }^{3} \mathrm{~F} 2$ & $+\quad 5 \% 1{ }^{3} \mathrm{D}$ \\
\hline 39120.48 & 39143.09 & -22.61 & 7 & 5 & 0.34 & 0.37 & 0.50 & $\left.52 \% 1\right|^{1} \mathrm{D} 2$ & $+\left.15 \% 1\right|^{3} \mathrm{~F} 2$ & $+13 \% 1{ }^{3} \mathrm{P} 2$ \\
\hline
\end{tabular}




$\begin{array}{rrrrrrrrrrrr}27670.52 & 27674.18 & -3.66 & 30 & 25 & 0.14 & 0.15 & 0.20 & 62 \% 1 \mid{ }^{3} \mathrm{D} & +16 \% 1 \mid{ }^{3} \mathrm{P} 1 & +8 \% 1 \mid{ }^{3} \mathrm{P} 2 \\ 22138.98 & 22109.19 & 29.80 & 25 & 20 & 0.17 & 0.13 & 0.22 & 69 \% 1 \mid{ }^{3} \mathrm{~F} 2 & +11 \% 1 \mid{ }^{1} \mathrm{D} 2 & +10 \% 1 \mid{ }^{3} \mathrm{P} 2 \\ 19128.73 & 19128.33 & 0.40 & 25 & 21 & 0.16 & 0.13 & 0.21 & 36 \% 1 \mid{ }^{5} \mathrm{D} & +27 \% 1 \mid{ }^{3} \mathrm{P} 2 & +11 \% 1{ }^{3} \mathrm{P} 1 \\ 5178.26 & 5175.30 & 2.96 & 30 & 29 & 0.15 & 0.12 & 0.19 & 61 \% 1 \mid{ }^{5} \mathrm{D} & +14 \% 1 \mid{ }^{3} \mathrm{P} 2 & +12 \% 1 \mid{ }^{3} \mathrm{P} 1\end{array}$

\begin{tabular}{|c|c|c|c|c|c|c|c|}
\hline \multicolumn{8}{|l|}{$J=3$} \\
\hline- & 121257.61 & - & & & & & \\
\hline - & 105058.13 & - & & & & & \\
\hline - & 102258.08 & - & & & & & \\
\hline- & 98423.21 & - & & & & & \\
\hline - & 94125.65 & - & & & & & \\
\hline & 90533.25 & - & & & & & \\
\hline 88009.29 & 88038.60 & -29.31 & 10 & 8 & 0.08 & 0.06 & 0.12 \\
\hline 85249.67 & 85246.43 & 3.24 & 9 & 5 & 0.10 & 0.05 & 0.15 \\
\hline 82458.31 & 82459.68 & -1.37 & 14 & 8 & 0.10 & 0.06 & 0.13 \\
\hline 79208.51 & 79197.28 & 11.23 & 19 & 15 & 0.07 & 0.06 & 0.10 \\
\hline 76493.69 & 76489.38 & 4.31 & 20 & 18 & 0.05 & 0.05 & 0.08 \\
\hline 74545.24 & 74515.75 & 29.49 & 19 & 14 & 0.07 & 0.06 & 0.10 \\
\hline 69741.38 & 69749.00 & -7.62 & 21 & 16 & 0.06 & 0.05 & 0.09 \\
\hline 66110.16 & 66119.79 & -9.64 & 25 & 19 & 0.06 & 0.06 & 0.09 \\
\hline 59232.65 & 59244.73 & -12.08 & 24 & 22 & 0.07 & 0.03 & 0.09 \\
\hline 54464.11 & 54448.32 & 15.79 & 23 & 17 & 0.07 & 0.05 & 0.10 \\
\hline 52259.88 & 52275.11 & -15.23 & 16 & 11 & 0.11 & 0.13 & 0.16 \\
\hline 35440.07 & 35425.36 & 14.71 & 29 & 19 & 0.16 & 0.12 & 0.20 \\
\hline 31975.85 & 31998.13 & -22.28 & 10 & 10 & 0.11 & 0.10 & 0.14 \\
\hline 30693.33 & 30715.97 & -22.65 & 31 & 21 & 0.16 & 0.11 & 0.19 \\
\hline 28959.73 & 28961.26 & -1.53 & 43 & 27 & 0.13 & 0.12 & 0.19 \\
\hline 21190.03 & 21192.02 & -1.99 & 39 & 31 & 0.13 & 0.11 & 0.18 \\
\hline 5824.11 & 5806.94 & 17.17 & 39 & 29 & 0.14 & 0.10 & 0.17 \\
\hline
\end{tabular}

\begin{tabular}{|c|c|c|}
\hline$\% 2 \mid\left({ }^{2} \mathrm{D} 1\right)^{3} \mathrm{D}$ & $+10 \% 2 \mid\left({ }^{2} \mathrm{D} 3\right)^{3} \mathrm{I}$ & \\
\hline 2) $\left({ }^{2} \mathrm{G} 1\right)^{3} \mathrm{G}$ & $23 \% 2 \mid\left({ }^{2} \mathrm{~F} 2\right)^{1} \mathrm{~F}$ & $+\quad 7 \% 22\left({ }^{4} \mathrm{~F}\right){ }^{3} \mathrm{~F}$ \\
\hline $2\left({ }^{2} \mathrm{D} 2\right)^{3} \mathrm{D}$ & $17 \% 22\left({ }^{2} \mathrm{G} 1\right)^{3} \mathrm{G}$ & $+13 \% 2\left({ }^{2} \mathrm{~F} 1\right)^{1} \mathrm{~F}$ \\
\hline $2 \mid\left({ }^{2} \mathrm{D} 2\right)^{3} \mathrm{D}$ & $+20 \% 2\left({ }^{2} \mathrm{~F} 1\right)^{1} \mathrm{~F}$ & $+13 \% 2 \mid\left({ }^{2} \mathrm{D} 3\right)^{3} \mathrm{D}$ \\
\hline $4 \% 2\left({ }^{4} \mathrm{~F}\right){ }^{3} \mathrm{~F}$ & $+10 \% 2\left({ }^{2} \mathrm{G} 1\right)^{3} \mathrm{G}$ & $+\quad 7 \% 2\left({ }^{2} \mathrm{D} 2\right)^{3} \mathrm{D}$ \\
\hline $6 \% 2\left({ }^{2} \mathrm{~F} 1\right)^{1} \mathrm{~F}$ & $+26 \% 2\left({ }^{2} \mathrm{~F} 1\right)^{3} \mathrm{~F}$ & $+13 \% 2\left({ }^{4} \mathrm{D}\right){ }^{3} \mathrm{D}$ \\
\hline $702\left({ }^{2} \mathrm{~F} 2\right){ }^{1} \mathrm{~F}$ & $+18 \% 2\left({ }^{2} \mathrm{G} 2\right)^{3} \mathrm{G}$ & $+13 \% 2\left({ }^{4} \mathrm{~F}\right){ }^{3} \mathrm{~F}$ \\
\hline $4 \% 2 \mid\left({ }^{2} \mathrm{~F} 2\right)^{3} \mathrm{~F}$ & $+12 \% 2\left({ }^{4} \mathrm{~F}\right){ }^{5} \mathrm{~F}$ & $+\quad 9 \% 2\left({ }^{2} \mathrm{D} 2\right)^{3} \mathrm{D}$ \\
\hline$\% 2$ & $+14 \% 2\left({ }^{4} \mathrm{~F}\right){ }^{5} \mathrm{~F}$ & $+12 \% 2\left({ }^{4} \mathrm{G}\right){ }^{3} \mathrm{G}$ \\
\hline $9 \% 2 \mid\left({ }^{2} \mathrm{G} 2\right)^{3} \mathrm{G}$ & $+18 \% 2\left({ }^{4} \mathrm{G}\right){ }^{3} \mathrm{G}$ & $+\quad 9 \% 2\left({ }^{4} \mathrm{~F}\right){ }^{3} \mathrm{~F}$ \\
\hline $2 \% 2 \%\left({ }^{4} \mathrm{D}\right){ }^{3} \mathrm{D}$ & $+21 \% 2\left({ }^{2} \mathrm{~F} 1\right)^{3} \mathrm{~F}$ & $8 \% 2\left({ }^{2} \mathrm{G} 2\right)^{3} \mathrm{G}$ \\
\hline $39 \% 2 \mid\left({ }^{4} \mathrm{~F}\right){ }^{5} \mathrm{~F}$ & $+\quad 13 \% 2 \mid\left({ }^{4} \mathrm{D}\right){ }^{3} \mathrm{D}$ & $+\quad 8 \% 2\left({ }^{2} \mathrm{~F} 2\right)^{3} \mathrm{~F}$ \\
\hline $11 \% 2 \mid\left({ }^{4} \mathrm{G}\right){ }^{3} \mathrm{G}$ & $+23 \% 2\left({ }^{4} \mathrm{P}\right){ }^{5} \mathrm{P}$ & $+11 \% 2\left({ }^{2} \mathrm{~F} 1\right)^{1} \mathrm{~F}$ \\
\hline $36 \% 2 \mid\left({ }^{4} \mathrm{D}\right){ }^{5} \mathrm{D}$ & $+14 \% 2\left({ }^{2} \mathrm{D} 3\right)^{3} \mathrm{D}$ & $+11 \% 2 \mid\left({ }^{4} \mathrm{G}\right){ }^{3} \mathrm{G}$ \\
\hline $50 \% 2 \mid\left({ }^{4} \mathrm{G}\right){ }^{5} \mathrm{G}$ & $+14 \% 2\left({ }^{4} \mathrm{P}\right){ }^{5} \mathrm{P}$ & $+13 \% 2\left({ }^{4} \mathrm{D}\right){ }^{5} \mathrm{D}$ \\
\hline $21 \% 2\left({ }^{4} \mathrm{D}\right){ }^{5} \mathrm{D}$ & $18 \% 2\left({ }^{4} \mathrm{G}\right){ }^{5} \mathrm{G}$ & $+15 \% 2\left({ }^{4} \mathrm{P}\right){ }^{5} \mathrm{P}$ \\
\hline $5 \% 1{ }^{3} \mathrm{~F} 1$ & $+\left.21 \% 1\right|^{1} \mathrm{~F}$ & $+8 \% 11^{3}$ \\
\hline$\left.2 \% 1\right|^{1}$ & $+\left.27 \% 1\right|^{3} \mathrm{~F} 1$ & $4 \% 1$ \\
\hline $38 \% 2 \mid\left({ }^{6} \mathrm{~S}\right)$ & $+10 \% 2\left({ }^{4} \mathrm{P}\right){ }^{5} \mathrm{P}$ & $+\left.\quad 1 \% 1\right|^{3} \mathrm{D}$ \\
\hline$\left.3 \% 1\right|^{3} \mathrm{G}$ & $+\left.34 \% 1\right|^{3} \mathrm{~F} 2$ & $+\quad 4 \% 1{ }^{1} \mathrm{~F}$ \\
\hline & $+15 \% 1{ }^{3} \mathrm{~F} 2$ & $+\quad 5 \% 1{ }^{5} \mathrm{D}$ \\
\hline$\left.5 \% 1\right|^{3} \mathrm{~F} 2$ & $+\left.34 \% 1\right|^{3} \mathrm{G}$ & $+12 \% 1{ }^{3} \mathrm{~F} 1$ \\
\hline & $+\quad 4 \% 1{ }^{3} \mathrm{~F} 2$ & $\left.3 \% 1\right|^{3} \mathrm{D}$ \\
\hline
\end{tabular}

$\mathrm{J}=4$

109222.52

103668.41

$96369.72-$

$\begin{array}{llllllll}90497.75 & 90507.86 & -10.11 & 13 & 11 & 0.08 & 0.06 & 0.12\end{array}$

$\begin{array}{llllllll}87782.64 & 87808.04 & -25.40 & 17 & 13 & 0.08 & 0.09 & 0.11\end{array}$

$\begin{array}{llllllll}84734.80 & 84739.47 & -4.67 & 16 & 9 & 0.10 & 0.05 & 0.13\end{array}$

$\begin{array}{llllllll}84005.15 & 83978.60 & 26.55 & 12 & 8 & 0.09 & 0.05 & 0.12\end{array}$

$\begin{array}{llllllll}79502.00 & 79541.75 & -39.75 & 19 & 12 & 0.09 & 0.06 & 0.12\end{array}$

$\begin{array}{llllllll}74057.35 & 74031.73 & 25.62 & 21 & 16 & 0.07 & 0.04 & 0.09\end{array}$

$\begin{array}{llllllll}71191.62 & 71183.12 & 8.50 & 26 & 22 & 0.05 & 0.04 & 0.00\end{array}$

$\begin{array}{llllllll}63912.64 & 63908.40 & 4.24 & 20 & 17 & 0.07 & 0.05 & 0.09\end{array}$

$\begin{array}{llllllll}58041.98 & 58046.18 & -4.20 & 22 & 17 & 0.08 & 0.06 & 0.11\end{array}$

$\begin{array}{llllllll}53982.00 & 53959.82 & 22.18 & 10 & 8 & 0.23 & 0.13 & 0.30\end{array}$

$\begin{array}{llllllll}46354.20 & 46359.31 & -5.11 & 20 & 11 & 0.18 & 0.17 & 0.24\end{array}$

$\begin{array}{llllllll}37387.78 & 37368.84 & 18.94 & 23 & 15 & 0.19 & 0.21 & 0.30\end{array}$

$\begin{array}{llllllll}27940.65 & 27948.34 & -7.69 & 36 & 29 & 0.14 & 0.12 & 0.19\end{array}$

$\begin{array}{rrrrrrrr}25969.65 & 25963.22 & 6.43 & 30 & 26 & 0.15 & 0.13 & 0.20\end{array}$

$\begin{array}{llllllll}14546.32 & 14560.31 & -13.99 & 32 & 29 & 0.14 & 0.11 & 0.18\end{array}$

$\begin{array}{llllllll}0.00 & -8.39 & 8.39 & 26 & 23 & 0.17 & 0.12 & 0.18\end{array}$

$\mathrm{J}=5$

$\begin{array}{rrrrrrrr}- & 102116.73 & - & & & & & \\ 95628.69 & 95622.29 & 6.40 & 9 & 7 & 0.11 & 0.09 & 0.15 \\ 90123.22 & 90101.87 & 21.35 & 13 & 10 & 0.10 & 0.06 & 0.13 \\ 81605.68 & 81640.80 & -35.12 & 20 & 15 & 0.07 & 0.05 & 0.10\end{array}$

$85 \% 2\left|\left({ }^{2} \mathrm{G} 1\right)^{1} \mathrm{G}+4 \% 2\right|\left({ }^{2} \mathrm{~F} 2\right)^{3} \mathrm{~F}+3 \% 2 \mid\left({ }^{2} \mathrm{G} 2\right)^{3} \mathrm{G}$ $68 \% 2\left|\left({ }^{2} \mathrm{G} 1\right)^{3} \mathrm{G}+13 \% 2\right|\left({ }^{2} \mathrm{G} 2\right)^{1} \mathrm{G}+5 \% 2 \mid\left({ }^{4} \mathrm{~F}\right){ }^{3} \mathrm{~F}$ $45 \% 2\left|\left({ }^{4} \mathrm{~F}\right){ }^{3} \mathrm{~F}+19 \% 2\right|\left({ }^{2} \mathrm{G} 1\right)^{3} \mathrm{G}+18 \% 2 \mid\left({ }^{2} \mathrm{G} 2\right)^{1} \mathrm{G}$ $25 \% 2\left|\left({ }^{2} \mathrm{~F} 2\right)^{3} \mathrm{~F}+25 \% 2\right|\left({ }^{2} \mathrm{G} 2\right)^{1} \mathrm{G}+15 \% 2 \mid\left({ }^{4} \mathrm{~F}\right){ }^{3} \mathrm{~F}$ $34 \% 2\left|\left({ }^{2} \mathrm{~F} 1\right)^{3} \mathrm{~F}+24 \% 2\right|\left({ }^{2} \mathrm{G} 2\right)^{3} \mathrm{G}+8 \% 2 \mid\left({ }^{2} \mathrm{H}\right){ }^{3} \mathrm{H}$ $38 \% 2\left|\left({ }^{2} \mathrm{H}\right){ }^{3} \mathrm{H}+22 \% 2\right|\left({ }^{2} \mathrm{~F} 2\right)^{3} \mathrm{~F}+20 \% 2 \mid\left({ }^{4} \mathrm{~F}\right){ }^{3} \mathrm{~F}$ $26 \% 2\left|\left({ }^{2} \mathrm{~F} 1\right)^{3} \mathrm{~F}+22 \% 2\right|\left({ }^{2} \mathrm{G} 2\right)^{3} \mathrm{G}+13 \% 2 \mid\left({ }^{2} \mathrm{G} 2\right)^{1} \mathrm{G}$ $26 \% 2\left|\left({ }^{2} \mathrm{G} 2\right)^{3} \mathrm{G}+23 \% 2\right|\left({ }^{2} \mathrm{H}\right){ }^{3} \mathrm{H}+15 \% 2 \mid\left({ }^{4} \mathrm{G}\right){ }^{3} \mathrm{G}$ $32 \% 2\left|\left({ }^{4} \mathrm{~F}\right){ }^{5} \mathrm{~F}+23 \% 2\right|\left({ }^{4} \mathrm{G}\right){ }^{3} \mathrm{G}+15 \% 2 \mid\left({ }^{2} \mathrm{~F} 2\right)^{3} \mathrm{~F}$ $42 \% 2\left|\left({ }^{4} \mathrm{G}\right){ }^{3} \mathrm{G}+15 \% 2\right|\left({ }^{2} \mathrm{G} 2\right)^{3} \mathrm{G}+13 \% 2 \mid\left({ }^{2} \mathrm{~F} 1\right)^{3} \mathrm{~F}$ $65 \% 2\left|\left({ }^{4} \mathrm{D}\right){ }^{5} \mathrm{D}+22 \% 2\right|\left({ }^{4} \mathrm{G}\right){ }^{5} \mathrm{G}+6 \% 2 \mid\left({ }^{4} \mathrm{~F}\right){ }^{5} \mathrm{~F}$ $68 \% 2\left|\left({ }^{4} \mathrm{G}\right){ }^{5} \mathrm{G}+12 \% 2\right|\left({ }^{4} \mathrm{D}\right){ }^{5} \mathrm{D}+10 \% 2 \mid\left({ }^{4} \mathrm{~F}\right){ }^{5} \mathrm{~F}$ $\left.65 \% 1\right|^{1} \mathrm{G} 1+\left.16 \% 1\right|^{1} \mathrm{G} 2+7 \% 1{ }^{3} \mathrm{~F} 2$ $\left.71 \% 1\right|^{3} \mathrm{~F} 1+\left.7 \% 1\right|^{3} \mathrm{~F} 2+\left.7 \% 1\right|^{3} \mathrm{G}$ $\left.59 \% 1\right|^{1} \mathrm{G} 2+20 \% 1{ }^{3} \mathrm{~F} 2+\left.14 \% 1\right|^{3} \mathrm{H}$ $\left.59 \% 1\right|^{3} \mathrm{G}+\left.17 \% 1\right|^{3} \mathrm{~F} 2+\left.16 \% 1\right|^{1} \mathrm{G} 1$ $\left.56 \% 1\right|^{3} \mathrm{H}+\left.23 \% 1\right|^{3} \mathrm{~F} 2+\left.10 \% 1\right|^{3} \mathrm{~F} 1$ $\left.24 \% 1\right|^{3} \mathrm{H} \quad+\left.24 \% 1\right|^{3} \mathrm{G}+\left.15 \% 1\right|^{1} \mathrm{G} 2$ $\left.81 \% 1\right|^{5} \mathrm{D} \quad+\left.\quad 9 \% 1\right|^{3} \mathrm{~F} 2+\left.6 \% 1\right|^{3} \mathrm{~F} 1$

$87 \% 2\left|\left({ }^{2} \mathrm{G} 1\right)^{3} \mathrm{G}+8 \% 2\right|\left({ }^{2} \mathrm{G} 2\right)^{3} \mathrm{G}+3 \% 2 \mid\left({ }^{2} \mathrm{H}\right){ }^{1} \mathrm{H}$ $69 \% 2\left|\left({ }^{2} \mathrm{H}\right){ }^{1} \mathrm{H}+10 \% 2\right|\left({ }^{2} \mathrm{I}\right){ }^{3} \mathrm{I}+9 \% 2 \mid\left({ }^{4} \mathrm{G}\right){ }^{3} \mathrm{G}$ $46 \% 2\left|\left({ }^{2} \mathrm{H}\right){ }^{3} \mathrm{H}+30 \% 2\right|\left({ }^{2} \mathrm{G} 2\right)^{3} \mathrm{G}+8 \% 2 \mid\left({ }^{4} \mathrm{~F}\right){ }^{5} \mathrm{~F}$ $33 \% 2\left|\left({ }^{4} \mathrm{~F}\right){ }^{5} \mathrm{~F}+28 \% 2\right|\left({ }^{2} \mathrm{H}\right){ }^{3} \mathrm{H}+22 \% 2 \mid\left({ }^{2} \mathrm{G} 2\right)^{3} \mathrm{G}$ 


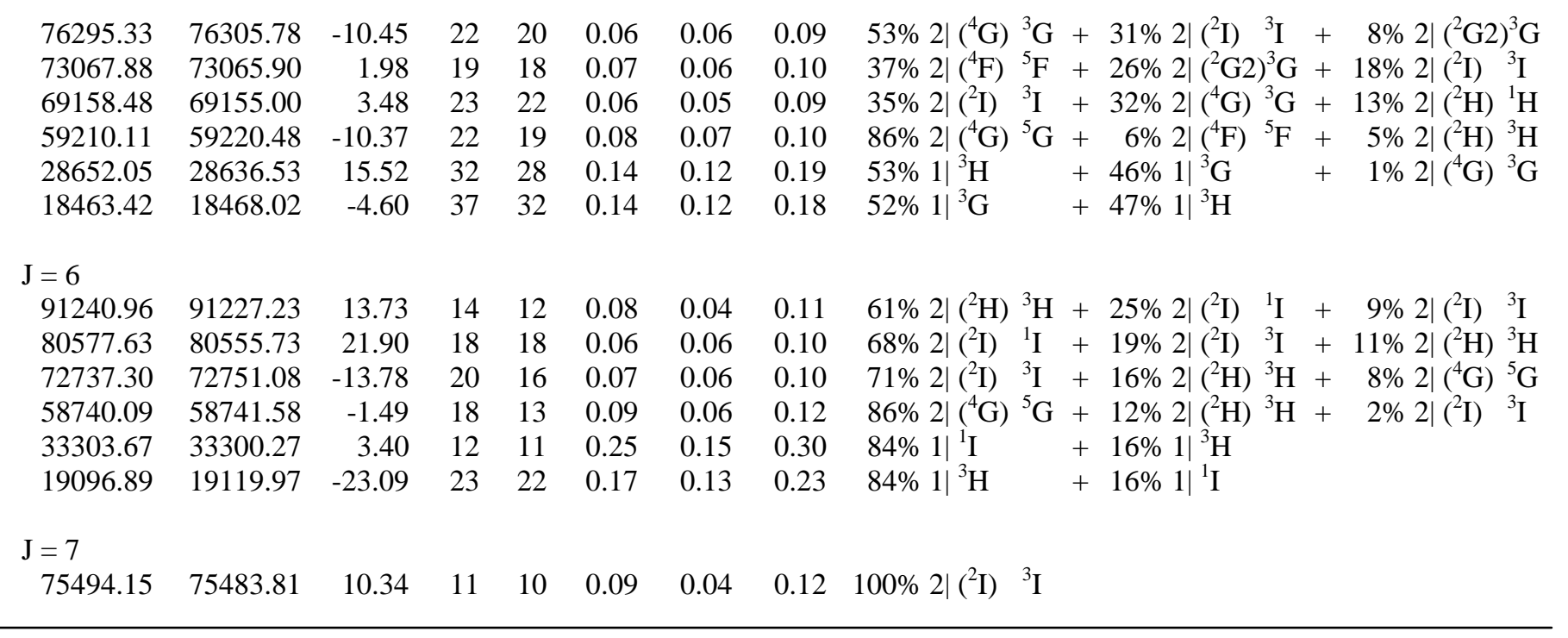


Table 3

Experimental and calculated energy levels $\left(\mathrm{cm}^{-1}\right)$ in the $5 \mathrm{~d}^{5} 6 \mathrm{p}$ and $5 \mathrm{~d}^{4} 6 \mathrm{~s} 6 \mathrm{p}$ configurations of Ir IV.

$\begin{array}{llllllllll}\mathrm{E}_{\mathrm{obs}} & \mathrm{E}_{\mathrm{calc}} & \Delta & \mathrm{N} & \mathrm{N}_{\mathrm{opt}} & \mathrm{rwmv}_{1} & \mathrm{rwmv}_{2} & \mathrm{Unc} & \text { Composition }\end{array}$

$\mathrm{J}=0$

- $\quad 204592.72$

- $203312.99 \quad-$

- 195852.09

- $\quad 195101.06$

- $\quad 183069.96-$

- 180255.66

- $\quad 174187.93$

- $\quad 173197.99$

- 170735.37 -

- $\quad 164573.60$

- $\quad 155133.42$

- $\quad 153150.27$

- 150706.04 -

- $\quad 146461.38$

- $\quad 142507.44$

- 135147.14

- 122586.06

$\mathrm{J}=1$

\begin{tabular}{|c|c|c|c|c|c|c|c|}
\hline - & 207252.96 & - & & & & & \\
\hline - & 206619.77 & - & & & & & \\
\hline - & 205826.71 & - & & & & & \\
\hline - & 204605.45 & - & & & & & \\
\hline - & 204449.85 & - & & & & & \\
\hline - & 202271.81 & - & & & & & \\
\hline - & 202069.00 & - & & & & & \\
\hline - & 200766.21 & - & & & & & \\
\hline - & 197786.43 & - & & & & & \\
\hline - & 195418.81 & - & & & & & \\
\hline - & 192858.84 & - & & & & & \\
\hline - & 192505.74 & - & & & & & \\
\hline- & 190739.70 & - & & & & & \\
\hline - & 190224.20 & - & & & & & \\
\hline - & 189016.86 & - & & & & & \\
\hline - & 188380.44 & - & & & & & \\
\hline - & 184839.62 & - & & & & & \\
\hline - & 181940.10 & - & & & & & \\
\hline - & 181052.13 & - & & & & & \\
\hline - & 178869.99 & - & & & & & \\
\hline - & 177466.43 & - & & & & & \\
\hline - & 176378.82 & - & & & & & \\
\hline - & 174151.32 & - & & & & & \\
\hline - & 173298.45 & - & & & & & \\
\hline - & 170988.34 & - & & & & & \\
\hline- & 167018.72 & - & & & & & \\
\hline - & 163869.83 & - & & & & & \\
\hline 16.70 & 163105.63 & 11.07 & 1 & 1 & 0.83 & 0.83 & 0.90 \\
\hline 84.04 & 161699.11 & -15.07 & 5 & 4 & 0.27 & 0.23 & 0.30 \\
\hline - & 160987.60 & - & & & & & \\
\hline
\end{tabular}

$36 \% 1\left|\left({ }^{2} \mathrm{D} 1\right){ }^{3} \mathrm{P}+13 \% 2\right|\left({ }^{3} \mathrm{D}^{4} \mathrm{D}\right){ }^{5} \mathrm{D}+8 \% 1 \mid\left({ }^{2} \mathrm{P}\right) \quad{ }^{3} \mathrm{P}$ $28 \% 2\left|\left({ }^{3} \mathrm{~F} 2{ }^{4} \mathrm{~F}\right){ }^{5} \mathrm{D}+14 \% 1\right|\left({ }^{2} \mathrm{D} 1\right){ }^{3} \mathrm{P}+12 \% 2 \mid\left({ }^{3} \mathrm{~F} 1{ }^{4} \mathrm{~F}\right){ }^{5} \mathrm{D}$ $29 \% 2$ 2 $\left({ }^{3} \mathrm{D}^{4} \mathrm{D}\right){ }^{5} \mathrm{D}+14 \% 2\left|\left({ }^{5} \mathrm{D}^{4} \mathrm{D}\right){ }^{5} \mathrm{D}+9 \% 2\right|\left({ }^{3} \mathrm{P} 2^{2} \mathrm{P}\right){ }^{3} \mathrm{P}$ $38 \% 2\left|\left({ }^{5} \mathrm{D}^{6} \mathrm{D}\right){ }^{5} \mathrm{D}+19 \% 2\right|\left({ }^{5} \mathrm{D}^{4} \mathrm{D}\right){ }^{3} \mathrm{P}+15 \% 2 \mid\left({ }^{5} \mathrm{D}^{4} \mathrm{D}\right){ }^{5} \mathrm{D}$ $59 \% 1\left|\left({ }^{2} \mathrm{P}\right){ }^{1} \mathrm{~S}+11 \% 1\right|\left({ }^{2} \mathrm{P}\right){ }^{3} \mathrm{P}+9 \% 1 \mid\left({ }^{2} \mathrm{D} 1\right){ }^{3} \mathrm{P}$ $28 \% 2\left|\left({ }^{3} \mathrm{P} 2{ }^{4} \mathrm{P}\right){ }^{5} \mathrm{D}+20 \% 2\right|\left({ }^{5} \mathrm{D}^{4} \mathrm{D}\right){ }^{3} \mathrm{P}+14 \% 2 \mid\left({ }^{3} \mathrm{P} 1{ }^{4} \mathrm{P}\right){ }^{5} \mathrm{D}$ $52 \% 1\left|\left({ }^{2} \mathrm{P}\right) \quad{ }^{3} \mathrm{P}+16 \% 1\right|\left({ }^{2} \mathrm{P}\right){ }^{1} \mathrm{~S}+8 \% 1 \mid\left({ }^{2} \mathrm{D} 1\right){ }^{3} \mathrm{P}$ $66 \% 1\left|\left({ }^{2} \mathrm{D} 2\right){ }^{3} \mathrm{P}+6 \% 1\right|\left({ }^{2} \mathrm{D} 1\right){ }^{3} \mathrm{P}+4 \% 1 \mid\left({ }^{2} \mathrm{P}\right){ }^{1} \mathrm{~S}$ $17 \% 2\left({ }^{5} \mathrm{D}^{4} \mathrm{D}\right){ }^{3} \mathrm{P}+16 \% 22\left({ }^{5} \mathrm{D}^{6} \mathrm{D}\right){ }^{7} \mathrm{~F}+8 \% 2 \mid\left({ }^{3} \mathrm{P}{ }^{2} \mathrm{P}\right){ }^{3} \mathrm{P}$ $51 \% 1\left|\left({ }^{2} \mathrm{D} 3\right){ }^{3} \mathrm{P}+19 \% 1\right|\left({ }^{4} \mathrm{~F}\right) \quad{ }^{5} \mathrm{D}+14 \% 1 \mid\left({ }^{4} \mathrm{P}\right){ }^{3} \mathrm{P}$ $50 \% 1\left|\left({ }^{4} \mathrm{~F}\right) \quad{ }^{5} \mathrm{D}+17 \% 1\right|\left({ }^{4} \mathrm{P}\right) \quad{ }^{3} \mathrm{P}+11 \% 2 \mid\left({ }^{5} \mathrm{D}^{6} \mathrm{D}\right){ }^{7} \mathrm{~F}$ $57 \% 2\left|\left({ }^{5} \mathrm{D}^{6} \mathrm{D}\right){ }^{7} \mathrm{~F}+10 \% 2\right|\left({ }^{3} \mathrm{P} 1{ }^{4} \mathrm{P}\right){ }^{5} \mathrm{D}+7 \% 1 \mid\left({ }^{4} \mathrm{~F}\right){ }^{5} \mathrm{D}$ $31 \% 1\left|\left({ }^{4} \mathrm{D}\right){ }^{3} \mathrm{P}+27 \% 1\right|\left({ }^{2} \mathrm{~S}\right){ }^{3} \mathrm{P}+11 \% 1 \mid\left({ }^{4} \mathrm{D}\right){ }^{5} \mathrm{D}$ $23 \% 1\left|\left({ }^{2} \mathrm{D} 3\right){ }^{3} \mathrm{P}+22 \% 1\right|\left({ }^{2} \mathrm{~S}\right) \quad{ }^{3} \mathrm{P}+19 \% 1 \mid\left({ }^{4} \mathrm{D}\right) \quad{ }^{5} \mathrm{D}$ $28 \% 1\left|(4 \mathrm{D}) \quad{ }^{3} \mathrm{P}+22 \% 1\right|\left({ }^{4} \mathrm{P}\right) \quad{ }^{3} \mathrm{P}+20 \% 1 \mid\left({ }^{4} \mathrm{P}\right) \quad{ }^{5} \mathrm{D}$ $40 \% 1\left|(4 \mathrm{D}) \quad{ }^{5} \mathrm{D}+24 \% 1\right|\left({ }^{4} \mathrm{P}\right) \quad{ }^{3} \mathrm{P}+15 \% 1 \mid\left({ }^{4} \mathrm{D}\right){ }^{3} \mathrm{P}$ $57 \% 1\left|(4 \mathrm{P}) \quad{ }^{5} \mathrm{D}+22 \% 1\right|\left({ }^{4} \mathrm{D}\right) \quad{ }^{5} \mathrm{D}+8 \% 1 \mid\left({ }^{2} \mathrm{~S}\right) \quad{ }^{3} \mathrm{P}$

$17 \% 1\left|\left({ }^{2} \mathrm{D} 1\right){ }^{1} \mathrm{P}+9 \% 1\right|\left({ }^{2} \mathrm{P}\right) \quad{ }^{1} \mathrm{P}+7 \% 2 \mid\left({ }^{3} \mathrm{P} 2{ }^{2} \mathrm{P}\right){ }^{3} \mathrm{D}$ $20 \% 1\left|\left({ }^{2} \mathrm{D} 1\right){ }^{1} \mathrm{P}+9 \% 1\right|\left({ }^{2} \mathrm{P}\right){ }^{1} \mathrm{P}+8 \% 2 \mid\left({ }^{3} \mathrm{G}^{4} \mathrm{G}\right){ }^{5} \mathrm{~F}$ $13 \% 2\left|\left({ }^{5} \mathrm{D}^{4} \mathrm{D}\right){ }^{5} \mathrm{P}+10 \% 2\right|\left({ }^{5} \mathrm{D}^{4} \mathrm{D}\right){ }^{3} \mathrm{D}+8 \% 2 \mid\left({ }^{3} \mathrm{D}^{4} \mathrm{D}\right){ }^{5} \mathrm{P}$ $27 \% 2\left({ }^{3} \mathrm{~F} 2{ }^{4} \mathrm{~F}\right){ }^{5} \mathrm{D}+13 \% 2 \mid\left({ }^{3} \mathrm{~F} 1{ }^{4} \mathrm{~F}\right){ }^{5} \mathrm{D}+8 \% 2\left({ }^{3} \mathrm{~F} 2{ }^{4} \mathrm{~F}\right){ }^{5} \mathrm{~F}$ $20 \% 2 \mid\left({ }^{3} \mathrm{D}^{4} \mathrm{D}\right){ }^{5} \mathrm{~F}+7 \% 2\left({ }^{3} \mathrm{P} 2^{2} \mathrm{P}\right){ }^{3} \mathrm{D}+7 \% 2\left({ }^{3} \mathrm{D}^{2} \mathrm{D}\right){ }^{3} \mathrm{D}$ $17 \% 2\left|\left({ }^{3} \mathrm{D}^{4} \mathrm{D}\right){ }^{5} \mathrm{D}+14 \% 2\right|\left({ }^{5} \mathrm{D}^{6} \mathrm{D}\right){ }^{5} \mathrm{~F}+13 \% 2 \mid\left({ }^{3} \mathrm{G}^{4} \mathrm{G}\right){ }^{5} \mathrm{~F}$ $18 \% 2\left|\left({ }^{3} \mathrm{G}^{4} \mathrm{G}\right){ }^{5} \mathrm{~F}+15 \% 2\right|\left({ }^{5} \mathrm{D}^{4} \mathrm{D}\right){ }^{5} \mathrm{~F}+11 \% 2 \mid\left({ }^{5} \mathrm{D}^{6} \mathrm{D}\right){ }^{5} \mathrm{~F}$ $46 \% 1\left|\left({ }^{2} \mathrm{D} 1\right){ }^{3} \mathrm{P}+9 \% 1\right|\left({ }^{2} \mathrm{D} 1\right){ }^{1} \mathrm{P}+9 \% 1 \mid\left({ }^{2} \mathrm{D} 3\right){ }^{3} \mathrm{P}$ $21 \% 2\left({ }^{3} \mathrm{D}^{4} \mathrm{D}\right){ }^{5} \mathrm{~F}+15 \% 2\left({ }^{3} \mathrm{~F} 2{ }^{4} \mathrm{~F}\right){ }^{5} \mathrm{~F}+14 \% 2\left({ }^{3} \mathrm{D}^{4} \mathrm{D}\right){ }^{5} \mathrm{D}$ $23 \% 2\left({ }^{5} \mathrm{D}^{4} \mathrm{D}\right){ }^{3} \mathrm{P}+13 \% 2\left({ }^{5} \mathrm{D}^{6} \mathrm{D}\right){ }^{5} \mathrm{D}+8 \% 2\left({ }^{3} \mathrm{~F} 2{ }^{4} \mathrm{~F}\right){ }^{5} \mathrm{~F}$ $22 \% 1\left|\left({ }^{2} \mathrm{P}\right) \quad{ }^{3} \mathrm{~S}+17 \% 1\right|\left({ }^{2} \mathrm{P}\right){ }^{3} \mathrm{P}+6 \% 2 \mid\left({ }^{3} \mathrm{P} 2{ }^{4} \mathrm{P}\right){ }^{5} \mathrm{P}$ $13 \% 1\left|\left({ }^{2} \mathrm{P}\right) \quad{ }^{3} \mathrm{~S}+12 \% 2\right|\left({ }^{3} \mathrm{P} 2{ }^{4} \mathrm{P}\right){ }^{5} \mathrm{P}+7 \% 1 \mid\left({ }^{2} \mathrm{P}\right){ }^{3} \mathrm{P}$ $16 \% 2\left|\left({ }^{5} \mathrm{D}^{6} \mathrm{D}\right){ }^{5} \mathrm{P}+10 \% 2\right|\left({ }^{3} \mathrm{P} 2{ }^{4} \mathrm{P}\right){ }^{5} \mathrm{P}+7 \% 2 \mid\left({ }^{3} \mathrm{~F} 2{ }^{4} \mathrm{~F}\right){ }^{5} \mathrm{~F}$ $27 \% 1\left|\left({ }^{2} \mathrm{D} 1\right){ }^{3} \mathrm{D}+18 \% 1\right|\left({ }^{2} \mathrm{P}\right) \quad{ }^{1} \mathrm{P}+16 \% 1 \mid\left({ }^{2} \mathrm{P}\right){ }^{3} \mathrm{~S}$ $30 \% 1\left|\left({ }^{2} \mathrm{P}\right) \quad{ }^{1} \mathrm{P}+14 \% 1\right|\left({ }^{2} \mathrm{D} 1\right){ }^{1} \mathrm{P}+7 \% 1 \mid\left({ }^{2} \mathrm{D} 2\right){ }^{1} \mathrm{P}$ $11 \% 1\left|\left({ }^{2} \mathrm{D} 1\right){ }^{3} \mathrm{D}+11 \% 1\right|\left({ }^{2} \mathrm{P}\right){ }^{3} \mathrm{~S}+10 \% 2 \mid\left({ }^{3} \mathrm{~F} 2{ }^{4} \mathrm{~F}\right){ }^{5} \mathrm{~F}$ $14 \% 2\left|\left({ }^{5} \mathrm{D}^{6} \mathrm{D}\right){ }^{5} \mathrm{P}+14 \% 2\right|\left({ }^{5} \mathrm{D}^{4} \mathrm{D}\right){ }^{5} \mathrm{D}+11 \% 2 \mid\left({ }^{5} \mathrm{D}^{6} \mathrm{D}\right){ }^{7} \mathrm{D}$ $30 \% 1\left|\left({ }^{2} \mathrm{P}\right) \quad{ }^{3} \mathrm{D}+13 \% 1\right|\left({ }^{2} \mathrm{D} 2\right){ }^{1} \mathrm{P}+13 \% 1 \mid\left({ }^{2} \mathrm{~F} 2\right){ }^{3} \mathrm{D}$ $20 \% 2\left|\left({ }^{3} \mathrm{P} 2{ }^{4} \mathrm{P}\right){ }^{5} \mathrm{D}+13 \% 2\right|\left({ }^{5} \mathrm{D}^{6} \mathrm{D}\right){ }^{7} \mathrm{~F}+12 \% 2 \mid\left({ }^{3} \mathrm{P} 1{ }^{4} \mathrm{P}\right){ }^{5} \mathrm{D}$ $24 \% 2\left|\left({ }^{5} \mathrm{D}^{6} \mathrm{D}\right){ }^{7} \mathrm{D}+19 \% 2\right|\left({ }^{5} \mathrm{D}^{4} \mathrm{D}\right){ }^{3} \mathrm{P}+8 \% 2 \mid\left({ }^{5} \mathrm{D}^{4} \mathrm{D}\right){ }^{5} \mathrm{D}$ $20 \% 1\left|\left({ }^{2} \mathrm{~F} 1\right){ }^{3} \mathrm{D}+18 \% 1\right|\left({ }^{2} \mathrm{D} 3\right){ }^{1} \mathrm{P}+9 \% 1 \mid\left({ }^{2} \mathrm{P}\right){ }^{3} \mathrm{D}$ $21 \% 1\left|\left({ }^{2} \mathrm{D} 2\right){ }^{3} \mathrm{P}+13 \% 1\right|\left({ }^{2} \mathrm{P}\right){ }^{3} \mathrm{D}+11 \% 1 \mid\left({ }^{2} \mathrm{D} 2\right){ }^{1} \mathrm{P}$ $23 \% 2\left|\left({ }^{5} \mathrm{D}^{4} \mathrm{D}\right){ }^{5} \mathrm{~F}+9 \% 2\right|\left({ }^{5} \mathrm{D}^{6} \mathrm{D}\right){ }^{5} \mathrm{~F}+6 \% 1 \mid\left({ }^{2} \mathrm{D} 2\right){ }^{3} \mathrm{D}$ $25 \% 1\left|\left({ }^{2} \mathrm{D} 2\right){ }^{3} \mathrm{D}+25 \% 1\right|\left({ }^{2} \mathrm{D} 2\right){ }^{3} \mathrm{P}+6 \% 1 \mid\left({ }^{2} \mathrm{D} 2\right){ }^{1} \mathrm{P}$ $21 \% 1\left|\left({ }^{2} \mathrm{P}\right) \quad{ }^{3} \mathrm{P}+14 \% 1\right|\left({ }^{2} \mathrm{P}\right){ }^{3} \mathrm{D}+12 \% 1 \mid\left({ }^{2} \mathrm{P}\right){ }^{3} \mathrm{~S}$ ${ }_{33 \% 1}\left({ }^{2} \mathrm{~S}\right) \quad{ }^{1} \mathrm{P}+15 \% 1\left|\left({ }^{2} \mathrm{P}\right) \quad{ }^{3} \mathrm{P}+7 \% 1\right|\left({ }^{4} \mathrm{~F}\right){ }^{3} \mathrm{D}$ $22 \% 1\left|\left({ }^{2} \mathrm{D} 3\right){ }^{3} \mathrm{P}+14 \% 1\right|\left({ }^{2} \mathrm{D} 3\right){ }^{3} \mathrm{D}+12 \% 1 \mid\left({ }^{4} \mathrm{~F}\right) \quad{ }^{5} \mathrm{D}$ $38 \% 1\left|\left({ }^{2} \mathrm{~F} 2\right){ }^{3} \mathrm{D}+9 \% 1\right|\left({ }^{2} \mathrm{~S}\right) \quad{ }^{3} \mathrm{P}+9 \% 1 \mid\left({ }^{4} \mathrm{P}\right) \quad{ }^{3} \mathrm{~S}$ $27 \% 1\left|\left({ }^{2} \mathrm{~F} 1\right){ }^{3} \mathrm{D}+9 \% 1\right|\left({ }^{4} \mathrm{~F}\right){ }^{3} \mathrm{D}+9 \% 1 \mid\left({ }^{4} \mathrm{P}\right){ }^{3} \mathrm{~S}$ $46 \% 2\left|\left({ }^{5} \mathrm{D}^{6} \mathrm{D}\right){ }^{7} \mathrm{D}+16 \% 2\right|\left({ }^{5} \mathrm{D}^{6} \mathrm{D}\right){ }^{5} \mathrm{P}+8 \% 2 \mid\left({ }^{3} \mathrm{P} 1{ }^{4} \mathrm{P}\right){ }^{5} \mathrm{P}$ 


\begin{tabular}{|c|c|c|c|c|c|c|c|}
\hline - & 159538.61 & - & & & & & \\
\hline- & 157688.41 & - & & & & & \\
\hline - & 155976.10 & - & & & & & \\
\hline- & 154222.32 & - & & & & & \\
\hline 153731.32 & 153674.47 & 56.85 & 2 & 2 & 0.31 & 0.32 & 0.30 \\
\hline 151872.11 & 151837.34 & 34.77 & 3 & 3 & 0.51 & 0.63 & 0.70 \\
\hline 150748.58 & 150774.66 & -26.08 & 2 & 2 & 0.30 & 0.12 & 0.30 \\
\hline 148637.14 & 148648.53 & -11.39 & 4 & 4 & 0.28 & 0.18 & 0.30 \\
\hline- & 146450.96 & - & & & & & \\
\hline 144347.40 & 144355.88 & -8.48 & 7 & 6 & 0.20 & 0.16 & 0.22 \\
\hline- & 143250.63 & - & & & & & \\
\hline 140678.86 & 140707.31 & -28.45 & 8 & 7 & 0.16 & 0.11 & 0.19 \\
\hline 137142.67 & 137151.46 & -8.79 & 8 & 5 & 0.17 & 0.15 & 0.21 \\
\hline 135950.44 & 135929.00 & 21.44 & 10 & 8 & 0.13 & 0.10 & 0.17 \\
\hline 135484.56 & 135446.79 & 37.77 & 6 & 6 & 0.18 & 0.08 & 0.20 \\
\hline- & 131139.72 & - & & & & & \\
\hline 127824.31 & 127783.62 & 40.69 & 8 & 8 & 0.11 & 0.08 & 0.14 \\
\hline 121359.77 & 121362.28 & -2.51 & 8 & 8 & 0.10 & 0.08 & 0.14 \\
\hline 118786.27 & 118810.01 & -23.74 & 7 & 7 & 0.10 & 0.06 & 0.13 \\
\hline \multicolumn{8}{|l|}{$\mathrm{J}=2$} \\
\hline - & 201282.55 & - & & & & & \\
\hline - & 200725.29 & - & & & & & \\
\hline - & 199762.07 & - & & & & & \\
\hline - & 199516.60 & - & & & & & \\
\hline - & 198751.06 & - & & & & & \\
\hline - & 198523.96 & - & & & & & \\
\hline - & 197253.90 & - & & & & & \\
\hline - & 194950.72 & - & & & & & \\
\hline - & 193200.37 & - & & & & & \\
\hline- & 191513.39 & - & & & & & \\
\hline - & 191133.49 & - & & & & & \\
\hline - & 190834.77 & - & & & & & \\
\hline - & 189646.22 & - & & & & & \\
\hline - & 189157.65 & - & & & & & \\
\hline - & 187856.15 & - & & & & & \\
\hline - & 185947.45 & - & & & & & \\
\hline - & 184662.88 & - & & & & & \\
\hline - & 183254.06 & - & & & & & \\
\hline - & 181256.55 & - & & & & & \\
\hline - & 180315.97 & - & & & & & \\
\hline - & 179521.28 & - & & & & & \\
\hline - & 177948.38 & - & & & & & \\
\hline - & 176492.42 & - & & & & & \\
\hline - & 173587.47 & - & & & & & \\
\hline - & 173035.82 & - & & & & & \\
\hline - & 171571.23 & - & & & & & \\
\hline - & 168991.08 & - & & & & & \\
\hline- & 167497.30 & - & & & & & \\
\hline - & 166017.19 & - & & & & & \\
\hline 165317.00 & 165321.62 & -4.62 & 7 & 5 & 0.40 & 0.22 & 0.40 \\
\hline 164909.90 & 164909.18 & 0.72 & 7 & 5 & 0.28 & 0.29 & 0.40 \\
\hline- & 163391.40 & - & & & & & \\
\hline 162134.97 & 162154.03 & -19.06 & 5 & 4 & 0.43 & 0.46 & 0.50 \\
\hline 161662.48 & 161722.06 & -59.58 & 1 & 1 & 0.88 & 0.88 & 0.90 \\
\hline 161062.97 & 161019.63 & 43.34 & 9 & 8 & 0.29 & 0.23 & 0.40 \\
\hline
\end{tabular}

$35 \% 1\left|\left({ }^{4} \mathrm{~F}\right) \quad{ }^{3} \mathrm{D}+14 \% 1\right|\left({ }^{2} \mathrm{~F} 2\right) \quad{ }^{3} \mathrm{D}+11 \% 1 \mid\left({ }^{4} \mathrm{P}\right) \quad{ }^{3} \mathrm{P}$ $30 \% 1\left|\left({ }^{2} \mathrm{D} 2\right){ }^{3} \mathrm{D}+18 \% 1\right|\left({ }^{4} \mathrm{~F}\right){ }^{5} \mathrm{D}+9 \% 1 \mid\left({ }^{2} \mathrm{D} 2\right){ }^{1} \mathrm{P}$ $70 \% 2\left|\left({ }^{5} \mathrm{D}^{6} \mathrm{D}\right){ }^{7} \mathrm{~F}+9 \% 2\right|\left({ }^{3} \mathrm{P} 1{ }^{4} \mathrm{P}\right){ }^{5} \mathrm{D}+5 \% 2 \mid\left({ }^{3} \mathrm{P} 2{ }^{4} \mathrm{P}\right){ }^{5} \mathrm{D}$ $20 \% 1\left|\left({ }^{4} \mathrm{~F}\right) \quad{ }^{5} \mathrm{D}+16 \% 1\right|\left({ }^{4} \mathrm{P}\right) \quad{ }^{3} \mathrm{D}+11 \% 1 \mid\left({ }^{4} \mathrm{D}\right) \quad{ }^{3} \mathrm{D}$ $18 \% 1\left|\left({ }^{2} \mathrm{~S}\right) \quad{ }^{3} \mathrm{P}+11 \% 1\right|\left({ }^{4} \mathrm{~F}\right) \quad{ }^{5} \mathrm{D}+7 \% 1 \mid\left({ }^{4} \mathrm{P}\right) \quad{ }^{3} \mathrm{P}$ $12 \% 1\left|\left({ }^{4} \mathrm{~F}\right) \quad{ }^{5} \mathrm{~F}+12 \% 1\right|\left({ }^{4} \mathrm{P}\right) \quad{ }^{3} \mathrm{P}+10 \% 1 \mid\left({ }^{4} \mathrm{D}\right) \quad{ }^{3} \mathrm{D}$ $13 \% 1\left|\left({ }^{2} \mathrm{~S}\right) \quad{ }^{3} \mathrm{P}+12 \% 1\right|\left({ }^{4} \mathrm{P}\right) \quad{ }^{5} \mathrm{P}+11 \% 1 \mid\left({ }^{4} \mathrm{D}\right){ }^{5} \mathrm{P}$ $26 \% 1\left|\left({ }^{4} \mathrm{D}\right) \quad{ }^{5} \mathrm{D}+11 \% 1\right|\left({ }^{2} \mathrm{D} 3\right){ }^{3} \mathrm{D}+9 \% 1 \mid\left({ }^{4} \mathrm{P}\right) \quad{ }^{5} \mathrm{D}$ $25 \% 1\left|\left({ }^{2} \mathrm{D} 3\right){ }^{3} \mathrm{P}+9 \% 1\right|\left({ }^{4} \mathrm{P}\right){ }^{3} \mathrm{P}+9 \% 1 \mid\left({ }^{4} \mathrm{G}\right){ }^{5} \mathrm{~F}$ ${ }_{43 \% 1} 1\left({ }^{4} \mathrm{P}\right) \quad{ }^{3} \mathrm{D}+9 \% 1\left|\left({ }^{4} \mathrm{D}\right) \quad{ }^{5} \mathrm{P}+8 \% 1\right|\left({ }^{4} \mathrm{~F}\right) \quad{ }^{3} \mathrm{D}$ $22 \% 1\left|\left({ }^{4} \mathrm{D}\right) \quad{ }^{3} \mathrm{P}+17 \% 1\right|\left({ }^{4} \mathrm{G}\right){ }^{5} \mathrm{~F}+8 \% 1 \mid\left({ }^{2} \mathrm{~S}\right){ }^{3} \mathrm{P}$ ${ }_{41 \%} 1\left|\left({ }^{4} \mathrm{D}\right) \quad{ }^{5} \mathrm{P}+11 \% 1\right|\left({ }^{4} \mathrm{P}\right) \quad{ }^{3} \mathrm{P}+10 \% 1 \mid\left({ }^{4} \mathrm{P}\right) \quad{ }^{5} \mathrm{P}$ $19 \% 1\left|\left({ }^{4} \mathrm{D}\right) \quad{ }^{5} \mathrm{D}+14 \% 1\right|\left({ }^{4} \mathrm{P}\right) \quad{ }^{5} \mathrm{P}+9 \% 1 \mid\left({ }^{2} \mathrm{D} 3\right){ }^{3} \mathrm{D}$ $18 \% 1\left|\left({ }^{4} \mathrm{P}\right) \quad{ }^{5} \mathrm{P}+18 \% 1\right|\left({ }^{4} \mathrm{D}\right) \quad{ }^{3} \mathrm{D}+15 \% 1 \mid\left({ }^{4} \mathrm{~F}\right) \quad{ }^{5} \mathrm{~F}$ $35 \% 1\left|\left({ }^{4} \mathrm{G}\right) \quad{ }^{5} \mathrm{~F}+20 \% 1\right|\left({ }^{2} \mathrm{~F} 1\right) \quad{ }^{3} \mathrm{D}+9 \% 1 \mid\left({ }^{4} \mathrm{P}\right) \quad{ }^{3} \mathrm{~S}$ ${ }_{40 \% 1}\left({ }^{4} \mathrm{D}\right) \quad{ }^{5} \mathrm{~F}+27 \% 1\left|\left({ }^{4} \mathrm{P}\right) \quad{ }^{5} \mathrm{D}+10 \% 1\right|\left({ }^{4} \mathrm{~F}\right) \quad{ }^{5} \mathrm{~F}$ $15 \% 1\left|\left({ }^{4} \mathrm{D}\right) \quad{ }^{5} \mathrm{~F}+11 \% 1\right|\left({ }^{4} \mathrm{D}\right) \quad{ }^{5} \mathrm{D}+11 \% 1 \mid\left({ }^{4} \mathrm{D}\right){ }^{3} \mathrm{P}$ $25 \% 1\left|\left({ }^{4} \mathrm{P}\right) \quad{ }^{5} \mathrm{D}+19 \% 1\right|\left({ }^{6} \mathrm{~S}\right) \quad{ }^{5} \mathrm{P}+18 \% 1 \mid\left({ }^{4} \mathrm{D}\right){ }^{5} \mathrm{~F}$ ${ }_{50 \% 1} 1\left|\left({ }^{6} \mathrm{~S}\right) \quad{ }^{5} \mathrm{P}+14 \% 1\right|\left({ }^{4} \mathrm{P}\right) \quad{ }^{5} \mathrm{P}+10 \% 1 \mid\left({ }^{4} \mathrm{P}\right) \quad{ }^{5} \mathrm{D}$

$18 \% 1\left|\left({ }^{2} \mathrm{D} 1\right){ }^{1} \mathrm{D}+9 \% 2\right|\left({ }^{3} \mathrm{H}^{4} \mathrm{H}\right){ }^{5} \mathrm{G}+7 \% 1 \mid\left({ }^{2} \mathrm{D} 1\right){ }^{3} \mathrm{P}$ $16 \% 1\left|\left({ }^{2} \mathrm{D} 1\right){ }^{1} \mathrm{D}+9 \% 2\left({ }^{3} \mathrm{H}^{4} \mathrm{H}\right){ }^{5} \mathrm{G}+9 \% 1\right|\left({ }^{2} \mathrm{D} 1\right){ }^{3} \mathrm{P}$ $15 \% 1\left|\left({ }^{2} \mathrm{D} 1\right){ }^{3} \mathrm{D}+10 \% 2\right|\left({ }^{5} \mathrm{D}^{4} \mathrm{D}\right){ }^{3} \mathrm{~F}+5 \% 1 \mid\left({ }^{2} \mathrm{D} 1\right){ }^{3} \mathrm{~F}$ $23 \% 1\left|\left({ }^{2} \mathrm{D} 1\right){ }^{3} \mathrm{D}+7 \% 2\right|\left({ }^{5} \mathrm{D}^{4} \mathrm{D}\right){ }^{3} \mathrm{~F}+6 \% 2 \mid\left({ }^{3} \mathrm{P} 2{ }^{4} \mathrm{P}\right){ }^{5} \mathrm{~S}$ $9 \% 2\left|\left({ }^{3} \mathrm{P} 2^{4} \mathrm{P}\right){ }^{5} \mathrm{P}+8 \% 2\right|\left({ }^{5} \mathrm{D}^{4} \mathrm{D}\right){ }^{3} \mathrm{~F}+6 \% 2 \mid\left({ }^{5} \mathrm{D}^{6} \mathrm{D}\right){ }^{5} \mathrm{~F}$ $17 \% 2$ 2 $\left({ }^{3} \mathrm{H}^{4} \mathrm{H}\right){ }^{5} \mathrm{G}+8 \% 2\left({ }^{5} \mathrm{D}^{4} \mathrm{D}\right){ }^{3} \mathrm{~F}+7 \% 2\left({ }^{5} \mathrm{D}^{6} \mathrm{D}\right){ }^{5} \mathrm{D}$ $21 \% 2\left({ }^{3} \mathrm{D}^{4} \mathrm{D}\right){ }^{5} \mathrm{~F}+9 \% 2\left({ }^{3} \mathrm{P} 2{ }^{4} \mathrm{P}\right){ }^{5} \mathrm{P}+5 \% 2\left({ }^{3} \mathrm{D}^{4} \mathrm{D}\right){ }^{5} \mathrm{P}$ $13 \% 2\left({ }^{5} \mathrm{D}^{4} \mathrm{D}\right){ }^{3} \mathrm{P}+13 \% 2\left({ }^{3} \mathrm{G}^{4} \mathrm{G}\right){ }^{5} \mathrm{~F}+13 \% 2\left({ }^{3} \mathrm{~F} 2{ }^{4} \mathrm{~F}\right){ }^{5} \mathrm{~F}$ $35 \% 1\left|\left({ }^{2} \mathrm{P}\right) \quad{ }^{1} \mathrm{D}+11 \% 1\right|\left({ }^{2} \mathrm{D} 1\right){ }^{3} \mathrm{P}+9 \% 1 \mid\left({ }^{2} \mathrm{P}\right){ }^{3} \mathrm{P}$ $13 \% 2\left|\left({ }^{3} \mathrm{~F} 2{ }^{4} \mathrm{~F}\right){ }^{5} \mathrm{G}+11 \% 2\right|\left({ }^{3} \mathrm{G}^{4} \mathrm{G}\right){ }^{5} \mathrm{G}+6 \% 1 \mid\left({ }^{2} \mathrm{P}\right) \quad{ }^{3} \mathrm{P}$ $17 \% 1\left|\left({ }^{2} \mathrm{P}\right){ }^{3} \mathrm{P}+9 \% 2\right|\left({ }^{3} \mathrm{G}^{4} \mathrm{G}\right){ }^{5} \mathrm{G}+8 \% 1 \mid\left({ }^{2} \mathrm{D} 1\right){ }^{1} \mathrm{D}$ $9 \% 1\left|\left({ }^{2} \mathrm{P}\right){ }^{3} \mathrm{D}+7 \% 1\right|\left({ }^{2} \mathrm{D} 1\right){ }^{3} \mathrm{~F}+7 \% 2 \mid\left({ }^{3} \mathrm{G}^{4} \mathrm{G}\right){ }^{5} \mathrm{G}$ $10 \% 2\left({ }^{3} \mathrm{D}^{4} \mathrm{D}\right){ }^{5} \mathrm{D}+6 \% 2\left({ }^{3} \mathrm{~F} 2{ }^{4} \mathrm{~F}\right){ }^{5} \mathrm{G}+6 \% 2\left({ }^{3} \mathrm{~F} 2{ }^{4} \mathrm{~F}\right){ }^{5} \mathrm{~F}$ $21 \% 1\left|\left({ }^{2} \mathrm{P}\right){ }^{3} \mathrm{D}+13 \% 1\right|\left({ }^{2} \mathrm{G} 1\right){ }^{3} \mathrm{~F}+9 \% 1 \mid\left({ }^{2} \mathrm{D} 1\right){ }^{1} \mathrm{D}$ $24 \% 2\left({ }^{3} \mathrm{P} 2{ }^{4} \mathrm{P}\right){ }^{5} \mathrm{D}+9 \% 2\left({ }^{3} \mathrm{D}^{4} \mathrm{D}\right){ }^{5} \mathrm{~F}+7 \% 2\left({ }^{5} \mathrm{D}^{4} \mathrm{D}\right){ }^{5} \mathrm{~F}$ $10 \% 2\left|\left({ }^{5} \mathrm{D}^{6} \mathrm{D}\right){ }^{5} \mathrm{D}+9 \% 2\right|\left({ }^{5} \mathrm{D}^{4} \mathrm{D}\right){ }^{5} \mathrm{D}+7 \% 2 \mid\left({ }^{5} \mathrm{D}^{4} \mathrm{D}\right){ }^{3} \mathrm{~F}$ $15 \% 2\left|\left({ }^{3} \mathrm{~F} 2{ }^{4} \mathrm{~F}\right){ }^{5} \mathrm{G}+10 \% 2\right|\left({ }^{5} \mathrm{D}^{6} \mathrm{D}\right){ }^{5} \mathrm{P}+9 \% 2 \mid\left({ }^{5} \mathrm{D}^{4} \mathrm{D}\right){ }^{5} \mathrm{P}$ $10 \% 2\left|\left({ }^{5} \mathrm{D}^{4} \mathrm{D}\right){ }^{5} \mathrm{P}+9 \% 2\right|\left({ }^{3} \mathrm{G}^{4} \mathrm{G}\right){ }^{5} \mathrm{G}+8 \% 2 \mid\left({ }^{5} \mathrm{D}^{6} \mathrm{D}\right){ }^{5} \mathrm{P}$ $19 \% 1\left|\left({ }^{2} \mathrm{G} 1\right){ }^{3} \mathrm{~F}+17 \% 1\right|\left({ }^{2} \mathrm{D} 1\right){ }^{3} \mathrm{P}+7 \% 1 \mid\left({ }^{2} \mathrm{P}\right){ }^{1} \mathrm{D}$ $17 \% 1\left|\left({ }^{2} \mathrm{D} 1\right){ }^{3} \mathrm{~F}+16 \% 1\right|\left({ }^{2} \mathrm{D} 2\right){ }^{1} \mathrm{D}+14 \% 1 \mid\left({ }^{2} \mathrm{G} 1\right){ }^{3} \mathrm{~F}$ $15 \% 1\left|\left({ }^{2} \mathrm{P}\right){ }^{3} \mathrm{P}+15 \% 1\right|\left({ }^{2} \mathrm{D} 2\right){ }^{1} \mathrm{D}+11 \% 1 \mid\left({ }^{2} \mathrm{D} 2\right){ }^{3} \mathrm{P}$ $27 \% 2\left|\left({ }^{5} \mathrm{D}^{4} \mathrm{D}\right){ }^{5} \mathrm{~F}+13 \% 2\right|\left({ }^{5} \mathrm{D}^{6} \mathrm{D}\right){ }^{5} \mathrm{~F}+8 \% 2 \mid\left({ }^{3} \mathrm{~F} 2{ }^{4} \mathrm{~F}\right){ }^{5} \mathrm{G}$ $23 \% 1\left|\left({ }^{2} \mathrm{D} 2\right){ }^{3} \mathrm{P}+18 \% 1\right|\left({ }^{2} \mathrm{D} 2\right){ }^{3} \mathrm{D}+5 \% 1 \mid\left({ }^{2} \mathrm{D} 3\right){ }^{1} \mathrm{D}$ $19 \% 1\left|\left({ }^{2} \mathrm{D} 2\right){ }^{1} \mathrm{D}+11 \% 1\right|\left({ }^{2} \mathrm{D} 1\right){ }^{3} \mathrm{~F}+7 \% 1 \mid\left({ }^{2} \mathrm{D} 2\right){ }^{3} \mathrm{D}$ $61 \% 2\left|\left({ }^{5} \mathrm{D}^{6} \mathrm{D}\right){ }^{7} \mathrm{P}+14 \% 2\right|\left({ }^{5} \mathrm{D}^{6} \mathrm{D}\right){ }^{7} \mathrm{D}+5 \% 2 \mid\left({ }^{3} \mathrm{P} 1{ }^{4} \mathrm{P}\right){ }^{5} \mathrm{P}$ $13 \% 1\left|\left({ }^{2} \mathrm{P}\right) \quad{ }^{3} \mathrm{D}+11 \% 1\right|\left({ }^{2} \mathrm{P}\right) \quad{ }^{1} \mathrm{D}+11 \% 1 \mid\left({ }^{2} \mathrm{P}\right){ }^{3} \mathrm{P}$ $29 \% 1\left|\left({ }^{2} \mathrm{~S}\right) \quad{ }^{3} \mathrm{P}+10 \% 1\right|\left({ }^{2} \mathrm{P}\right) \quad{ }^{3} \mathrm{D}+7 \% 1 \mid\left({ }^{2} \mathrm{~F} 1\right){ }^{1} \mathrm{D}$ $14 \% 1\left|\left({ }^{2} \mathrm{~F} 2\right){ }^{1} \mathrm{D}+12 \% 1\right|\left({ }^{4} \mathrm{~F}\right) \quad{ }^{3} \mathrm{~F}+11 \% 1 \mid\left({ }^{2} \mathrm{D} 2\right){ }^{3} \mathrm{D}$ $50 \% 2\left|\left({ }^{5} \mathrm{D}^{6} \mathrm{D}\right){ }^{7} \mathrm{D}+17 \% 2\right|\left({ }^{5} \mathrm{D}^{6} \mathrm{D}\right){ }^{7} \mathrm{P}+14 \% 2 \mid\left({ }^{5} \mathrm{D}^{6} \mathrm{D}\right){ }^{5} \mathrm{P}$ $15 \% 1\left|\left({ }^{2} \mathrm{G} 2\right){ }^{3} \mathrm{~F}+12 \% 1\right|\left({ }^{2} \mathrm{~F} 1\right){ }^{3} \mathrm{D}+12 \% 1 \mid\left({ }^{4} \mathrm{~F}\right){ }^{3} \mathrm{~F}$ $18 \% 1\left|\left({ }^{4} \mathrm{~F}\right) \quad{ }^{3} \mathrm{~F}+14 \% 1\right|\left({ }^{2} \mathrm{~F} 1\right) \quad{ }^{1} \mathrm{D}+12 \% 1 \mid\left({ }^{2} \mathrm{~S}\right) \quad{ }^{3} \mathrm{P}$ $15 \% 1\left|\left({ }^{2} \mathrm{~F} 1\right){ }^{3} \mathrm{D}+10 \% 1\right|\left({ }^{2} \mathrm{D} 2\right){ }^{3} \mathrm{D}+9 \% 1 \mid\left({ }^{4} \mathrm{~F}\right){ }^{3} \mathrm{D}$ $24 \% 1\left|\left({ }^{2} \mathrm{~F} 2\right){ }^{3} \mathrm{D}+9 \% 1\right|\left({ }^{4} \mathrm{~F}\right){ }^{3} \mathrm{~F}+7 \% 1 \mid\left({ }^{2} \mathrm{D} 2\right){ }^{1} \mathrm{D}$ $25 \% 1\left|\left({ }^{4} \mathrm{~F}\right) \quad{ }^{5} \mathrm{D}+7 \% 1\right|\left({ }^{2} \mathrm{D} 2\right){ }^{3} \mathrm{P}+7 \% 1 \mid\left({ }^{4} \mathrm{~F}\right){ }^{5} \mathrm{~F}$ $12 \% 1\left|\left({ }^{2} \mathrm{~F} 1\right){ }^{1} \mathrm{D}+12 \% 1\right|\left({ }^{2} \mathrm{G} 2\right){ }^{3} \mathrm{~F}+10 \% 1 \mid\left({ }^{2} \mathrm{~F} 2\right){ }^{3} \mathrm{D}$ 


$\begin{array}{crrrrrrr}- & 159602.82 & - & & & & & \\ 159160.81 & 159154.12 & 6.69 & 8 & 8 & 0.24 & 0.19 & 0.30 \\ 157099.59 & 157106.39 & -6.80 & 5 & 4 & 0.38 & 0.22 & 0.40 \\ 156429.22 & 156404.28 & 24.94 & 11 & 9 & 0.18 & 0.17 & 0.30 \\ 154852.06 & 154849.56 & 2.50 & 12 & 3 & 0.44 & 0.78 & 0.60 \\ 153193.13 & 153201.83 & -8.71 & 9 & 7 & 0.26 & 0.23 & 0.30 \\ 151248.97 & 151291.58 & -42.61 & 2 & 2 & 0.32 & 0.04 & 0.30 \\ 149264.53 & 149253.17 & 11.37 & 8 & 7 & 0.23 & 0.24 & 0.30 \\ 148685.19 & 148651.71 & 33.48 & 6 & 4 & 0.30 & 0.36 & 0.40 \\ 148135.70 & 148184.16 & -48.46 & 8 & 7 & 0.17 & 0.13 & 0.20 \\ 146330.41 & 146329.71 & 0.70 & 6 & 6 & 0.20 & 0.28 & 0.30 \\ 145359.05 & 145423.97 & -64.92 & 9 & 6 & 0.14 & 0.14 & 0.17 \\ 144626.15 & 144640.47 & -14.32 & 9 & 6 & 0.13 & 0.05 & 0.16 \\ 142865.10 & 142858.21 & 6.89 & 13 & 10 & 0.17 & 0.14 & 0.20 \\ 141466.77 & 141461.79 & 4.98 & 5 & 4 & 0.23 & 0.20 & 0.30 \\ 140473.23 & 140507.45 & -34.22 & 10 & 7 & 0.16 & 0.13 & 0.20 \\ 138612.56 & 138585.42 & 27.14 & 8 & 8 & 0.12 & 0.17 & 0.18 \\ 137229.06 & 137194.74 & 34.32 & 12 & 11 & 0.10 & 0.10 & 0.14 \\ 134707.64 & 134715.88 & -8.24 & 13 & 12 & 0.13 & 0.12 & 0.17 \\ 133204.10 & 133215.89 & -11.79 & 11 & 8 & 0.15 & 0.10 & 0.18 \\ 132468.31 & 132506.83 & -38.52 & 8 & 5 & 0.10 & 0.04 & 0.14 \\ 130875.24 & 130921.74 & -46.50 & 10 & 7 & 0.10 & 0.12 & 0.15 \\ 122746.29 & 122756.59 & -10.30 & 9 & 9 & 0.08 & 0.05 & 0.11 \\ 120891.70 & 120909.55 & -17.85 & 11 & 10 & 0.09 & 0.06 & 0.11 \\ 118527.90 & 118542.40 & -14.50 & 9 & 8 & 0.11 & 0.08 & 0.15 \\ 113807.41 & 113834.37 & -26.96 & 8 & 8 & 0.08 & 0.09 & 0.11 \\ 98528.30 & 98467.84 & 60.46 & 2 & 2 & 0.25 & 0.17 & 0.30\end{array}$

$\mathrm{J}=3$

\begin{tabular}{|c|c|c|c|c|c|c|c|}
\hline - & 202593.25 & - & & & & & \\
\hline - & 202234.15 & - & & & & & \\
\hline - & 201893.50 & - & & & & & \\
\hline - & 200274.59 & - & & & & & \\
\hline - & 199331.16 & - & & & & & \\
\hline - & 198841.27 & - & & & & & \\
\hline - & 198013.43 & - & & & & & \\
\hline - & 196381.30 & - & & & & & \\
\hline - & 195672.19 & - & & & & & \\
\hline - & 193128.85 & - & & & & & \\
\hline - & 192074.17 & - & & & & & \\
\hline - & 189650.98 & - & & & & & \\
\hline - & 189186.65 & - & & & & & \\
\hline - & 188380.44 & - & & & & & \\
\hline - & 187284.55 & - & & & & & \\
\hline - & 186646.75 & - & & & & & \\
\hline - & 185463.53 & - & & & & & \\
\hline - & 184548.35 & - & & & & & \\
\hline- & 182952.44 & - & & & & & \\
\hline 179941.78 & 179931.46 & 10.32 & 3 & 3 & 0.51 & 0.66 & 0.70 \\
\hline- & 178516.88 & - & & & & & \\
\hline - & 177587.44 & - & & & & & \\
\hline - & 176850.64 & - & & & & & \\
\hline - & 175042.27 & - & & & & & \\
\hline - & 173502.98 & - & & & & & \\
\hline - & 172815.57 & - & & & & & \\
\hline 169683.24 & 169641.02 & 42.22 & 9 & 6 & 0.26 & 0.12 & 0.30 \\
\hline
\end{tabular}

$80 \% 2\left|\left({ }^{5} \mathrm{D}^{6} \mathrm{D}\right){ }^{7} \mathrm{~F}+5 \% 2\right|\left({ }^{3} \mathrm{P} 1{ }^{4} \mathrm{P}\right){ }^{5} \mathrm{D}+3 \% 2 \mid\left({ }^{5} \mathrm{D}^{6} \mathrm{D}\right){ }^{5} \mathrm{D}$ $16 \% 1\left|\left({ }^{2} \mathrm{~F} 2\right){ }^{3} \mathrm{~F}+13 \% 1\right|\left({ }^{2} \mathrm{D} 2\right){ }^{3} \mathrm{D}+11 \% 1 \mid\left({ }^{2} \mathrm{D} 2\right){ }^{3} \mathrm{P}$ $17 \% 1\left|\left({ }^{2} \mathrm{D} 2\right){ }^{3} \mathrm{~F}+7 \% 1\right|\left({ }^{2} \mathrm{~F} 1\right){ }^{1} \mathrm{D}+7 \% 1 \mid\left({ }^{4} \mathrm{D}\right) \quad 3 \mathrm{~F}$ $10 \% 1\left|\left({ }^{2} \mathrm{~F} 2\right){ }^{3} \mathrm{D}+10 \% 1\right|\left({ }^{4} \mathrm{~F}\right) \quad{ }^{3} \mathrm{D}+7 \% 1 \mid\left({ }^{2} \mathrm{~F} 1\right) \quad 3 \mathrm{~F}$ $19 \% 1\left|\left({ }^{4} \mathrm{D}\right) \quad{ }^{3} \mathrm{~F}+10 \% 1\right|\left({ }^{4} \mathrm{P}\right) \quad{ }^{3} \mathrm{P}+8 \% 1 \mid\left({ }^{2} \mathrm{~F} 2\right){ }^{1} \mathrm{D}$ $26 \% 1\left|\left({ }^{2} \mathrm{D} 3\right){ }^{3} \mathrm{D}+10 \% 1\right|\left({ }^{4} \mathrm{~F}\right) \quad{ }^{5} \mathrm{~F}+9 \% 1 \mid\left({ }^{2} \mathrm{D} 1\right){ }^{3} \mathrm{D}$ $19 \% 1\left|\left({ }^{4} \mathrm{P}\right) \quad{ }^{3} \mathrm{D}+13 \% 1\right|\left({ }^{4} \mathrm{~F}\right) \quad{ }^{5} \mathrm{~F}+12 \% 1 \mid\left({ }^{4} \mathrm{D}\right){ }^{5} \mathrm{P}$ $24 \% 1\left|\left({ }^{4} \mathrm{D}\right){ }^{3} \mathrm{P}+13 \% 1\right|\left({ }^{2} \mathrm{D} 3\right){ }^{3} \mathrm{~F}+6 \% 1 \mid\left({ }^{2} \mathrm{~F} 2\right){ }^{3} \mathrm{~F}$ $10 \% 1\left({ }^{4} \mathrm{P}\right) \quad{ }^{3} \mathrm{P}+8 \% 1\left|\left({ }^{4} \mathrm{~F}\right){ }^{5} \mathrm{D}+6 \% 1\right|\left({ }^{2} \mathrm{~F} 2\right){ }^{3} \mathrm{D}$ $11 \% 1\left|\left({ }^{4} \mathrm{D}\right) \quad{ }^{3} \mathrm{~F}+9 \% 1\right|\left({ }^{4} \mathrm{D}\right) \quad{ }^{5} \mathrm{D}+9 \% 1 \mid\left({ }^{4} \mathrm{P}\right){ }^{5} \mathrm{P}$ $20 \% 1\left|\left({ }^{2} \mathrm{D} 3\right){ }^{3} \mathrm{~F}+12 \% 1\right|\left({ }^{4} \mathrm{G}\right) \quad{ }^{3} \mathrm{~F}+10 \% 1 \mid\left({ }^{4} \mathrm{~F}\right) \quad{ }^{5} \mathrm{G}$ $19 \% 1\left|\left({ }^{4} \mathrm{G}\right) \quad{ }^{3} \mathrm{~F}+14 \% 1\right|\left({ }^{4} \mathrm{~F}\right) \quad{ }^{5} \mathrm{G}+10 \% 1 \mid\left({ }^{2} \mathrm{~F} 1\right) \quad{ }^{3} \mathrm{~F}$ $13 \% 1\left|\left({ }^{4} \mathrm{D}\right) \quad{ }^{5} \mathrm{P}+8 \% 1\right|\left({ }^{4} \mathrm{P}\right) \quad{ }^{5} \mathrm{~S}+6 \% 1 \mid\left({ }^{4} \mathrm{D}\right){ }^{3} \mathrm{D}$ $18 \% 1\left|\left({ }^{4} \mathrm{~F}\right) \quad{ }^{5} \mathrm{~F}+13 \% 1\right|\left({ }^{4} \mathrm{~F}\right) \quad{ }^{5} \mathrm{D}+12 \% 1 \mid\left({ }^{4} \mathrm{D}\right) \quad{ }^{3} \mathrm{P}$ $21 \% 1\left|\left({ }^{4} \mathrm{P}\right) \quad{ }^{5} \mathrm{P}+12 \% 1\right|\left({ }^{4} \mathrm{P}\right) \quad{ }^{5} \mathrm{D}+12 \% 1 \mid\left({ }^{4} \mathrm{D}\right) \quad{ }^{3} \mathrm{~F}$ $31 \% 1\left|\left({ }^{4} \mathrm{G}\right) \quad{ }^{5} \mathrm{~F}+10 \% 1\right|\left({ }^{4} \mathrm{P}\right) \quad{ }^{5} \mathrm{~S}+7 \% 1 \mid\left({ }^{4} \mathrm{D}\right){ }^{3} \mathrm{D}$ $22 \% 1\left|\left({ }^{4} \mathrm{D}\right) \quad{ }^{5} \mathrm{P}+13 \% 1\right|\left({ }^{4} \mathrm{P}\right) \quad{ }^{3} \mathrm{D}+8 \% 1 \mid\left({ }^{4} \mathrm{P}\right) \quad{ }^{5} \mathrm{P}$ $19 \% 1\left|\left({ }^{4} \mathrm{G}\right) \quad{ }^{5} \mathrm{G}+15 \% 1\right|\left({ }^{4} \mathrm{D}\right) \quad{ }^{5} \mathrm{D}+11 \% 1 \mid\left({ }^{2} \mathrm{D} 3\right){ }^{3} \mathrm{~F}$ $16 \% 1\left|\left({ }^{4} \mathrm{G}\right) \quad{ }^{5} \mathrm{~F}+9 \% 1\right|\left({ }^{4} \mathrm{P}\right) \quad{ }^{5} \mathrm{~S}+9 \% 1 \mid\left({ }^{4} \mathrm{~F}\right){ }^{5} \mathrm{G}$ $15 \% 1\left|\left({ }^{4} \mathrm{~F}\right) \quad{ }^{5} \mathrm{G}+13 \% 1\right|\left({ }^{4} \mathrm{P}\right) \quad{ }^{5} \mathrm{D}+13 \% 1 \mid\left({ }^{4} \mathrm{G}\right){ }^{3} \mathrm{~F}$ $16 \% 1\left|\left({ }^{4} \mathrm{D}\right) \quad{ }^{5} \mathrm{~F}+9 \% 1\right|\left({ }^{4} \mathrm{D}\right) \quad{ }^{3} \mathrm{D}+8 \% 1 \mid\left({ }^{2} \mathrm{D} 3\right){ }^{3} \mathrm{~F}$ $10 \% 1\left|\left({ }^{4} \mathrm{P}\right) \quad{ }^{5} \mathrm{D}+8 \% 1\right|\left({ }^{4} \mathrm{~F}\right) \quad{ }^{5} \mathrm{~F}+6 \% 1 \mid\left({ }^{4} \mathrm{D}\right){ }^{5} \mathrm{~F}$ $24 \% 1\left|\left({ }^{4} \mathrm{G}\right) \quad{ }^{5} \mathrm{G}+12 \% 1\right|\left({ }^{4} \mathrm{P}\right) \quad{ }^{5} \mathrm{~S}+8 \% 1 \mid\left({ }^{4} \mathrm{G}\right){ }^{3} \mathrm{~F}$ $30 \% 1\left|\left({ }^{4} \mathrm{D}\right) \quad{ }^{5} \mathrm{~F}+18 \% 1\right|\left({ }^{4} \mathrm{P}\right) \quad{ }^{5} \mathrm{D}+10 \% 1 \mid\left({ }^{6} \mathrm{~S}\right) \quad{ }^{5} \mathrm{P}$ ${ }_{49 \% 1} 1\left({ }^{6} \mathrm{~S}\right) \quad{ }^{5} \mathrm{P}+10 \% 1\left|\left({ }^{6} \mathrm{~S}\right) \quad{ }^{7} \mathrm{P}+7 \% 1\right|\left({ }^{4} \mathrm{D}\right){ }^{5} \mathrm{P}$ $14 \% 1\left|\left({ }^{4} \mathrm{G}\right) \quad{ }^{5} \mathrm{G}+12 \% 1\right|\left({ }^{2} \mathrm{~F} 1\right) \quad{ }^{3} \mathrm{~F}+10 \% 1 \mid\left({ }^{4} \mathrm{G}\right) \quad{ }^{3} \mathrm{~F}$ ${ }_{73 \% 1} 1\left|\left({ }^{6} \mathrm{~S}\right) \quad{ }^{7} \mathrm{P}+10 \% 1\right|\left({ }^{6} \mathrm{~S}\right) \quad{ }^{5} \mathrm{P}+7 \% 1 \mid\left({ }^{4} \mathrm{P}\right) \quad{ }^{5} \mathrm{P}$

$39 \% 1\left|\left({ }^{2} \mathrm{D} 1\right){ }^{1} \mathrm{~F}+15 \% 1\right|\left({ }^{2} \mathrm{D} 1\right){ }^{3} \mathrm{~F}+7 \% 1 \mid\left({ }^{2} \mathrm{P}\right) \quad{ }^{3} \mathrm{D}$ $12 \% 2\left|\left({ }^{3} \mathrm{D}^{4} \mathrm{D}\right){ }^{5} \mathrm{D}+8 \% 1\right|\left({ }^{2} \mathrm{D} 1\right){ }^{3} \mathrm{D}+6 \% 2 \mid\left({ }^{3} \mathrm{G}^{2} \mathrm{G}\right){ }^{1} \mathrm{~F}$ $39 \% 1\left|\left({ }^{2} \mathrm{D} 1\right){ }^{3} \mathrm{D}+7 \% 1\right|\left({ }^{2} \mathrm{D} 1\right){ }^{3} \mathrm{~F}+6 \% 1 \mid\left({ }^{2} \mathrm{D} 3\right){ }^{3} \mathrm{D}$ $12 \% 2\left|\left({ }^{3} \mathrm{H}^{4} \mathrm{H}\right){ }^{5} \mathrm{H}+11 \% 2\right|\left({ }^{3} \mathrm{G}^{2} \mathrm{G}\right){ }^{1} \mathrm{~F}+6 \% 2 \mid\left({ }^{3} \mathrm{G}^{2} \mathrm{G}\right){ }^{3} \mathrm{G}$ $16 \% 2\left|\left({ }^{3} \mathrm{H}^{4} \mathrm{H}\right){ }^{5} \mathrm{G}+10 \% 2\right|\left({ }^{5} \mathrm{D}^{4} \mathrm{D}\right){ }^{3} \mathrm{~F}+9 \% 2 \mid\left({ }^{5} \mathrm{D}^{6} \mathrm{D}\right){ }^{5} \mathrm{D}$ $11 \% 2\left({ }^{3} \mathrm{P} 2{ }^{4} \mathrm{P}\right){ }^{5} \mathrm{D}+8 \% 2\left({ }^{3} \mathrm{P} 1{ }^{4} \mathrm{P}\right){ }^{5} \mathrm{D}+6 \% 2\left({ }^{3} \mathrm{D}^{4} \mathrm{D}\right){ }^{5} \mathrm{P}$ $18 \% 2\left({ }^{3} \mathrm{D}^{4} \mathrm{D}\right){ }^{5} \mathrm{P}+12 \% 2\left({ }^{3} \mathrm{G}^{4} \mathrm{G}\right){ }^{5} \mathrm{~F}+9 \% 2\left({ }^{3} \mathrm{P} 2^{4} \mathrm{P}\right){ }^{5} \mathrm{D}$ $21 \% 2\left|\left({ }^{3} \mathrm{G}^{4} \mathrm{G}\right){ }^{5} \mathrm{G}+13 \% 2\right|\left({ }^{3} \mathrm{~F} 2{ }^{4} \mathrm{~F}\right){ }^{5} \mathrm{~F}+7 \% 2\left({ }^{3} \mathrm{D}^{4} \mathrm{D}\right){ }^{5} \mathrm{D}$ $13 \% 2\left|\left({ }^{3} \mathrm{~F} 2{ }^{4} \mathrm{~F}\right){ }^{5} \mathrm{~F}+13 \% 2\right|\left({ }^{5} \mathrm{D}^{4} \mathrm{D}\right){ }^{3} \mathrm{~F}+8 \% 2 \mid\left({ }^{3} \mathrm{~F} 2{ }^{4} \mathrm{~F}\right){ }^{5} \mathrm{G}$ $13 \% 2\left|\left({ }^{5} \mathrm{D}^{6} \mathrm{D}\right){ }^{5} \mathrm{D}+11 \% 2\right|\left({ }^{5} \mathrm{D}^{6} \mathrm{D}\right){ }^{5} \mathrm{~F}+10 \% 2 \mid\left({ }^{5} \mathrm{D}^{4} \mathrm{D}\right){ }^{5} \mathrm{D}$ $26 \% 2\left|\left({ }^{3} \mathrm{~F} 2{ }^{4} \mathrm{~F}\right){ }^{5} \mathrm{G}+7 \% 2\right|\left({ }^{3} \mathrm{D}^{4} \mathrm{D}\right){ }^{5} \mathrm{D}+6 \% 2\left({ }^{3} \mathrm{D}^{4} \mathrm{D}\right){ }^{5} \mathrm{P}$ $12 \% 2\left|\left({ }^{3} \mathrm{D}^{4} \mathrm{D}\right){ }^{5} \mathrm{~F}+10 \% 1\right|\left({ }^{2} \mathrm{D} 1\right){ }^{3} \mathrm{~F}+6 \% 2\left({ }^{5} \mathrm{D}^{6} \mathrm{D}\right){ }^{5} \mathrm{D}$ $26 \% 2\left({ }^{3} \mathrm{G}^{4} \mathrm{G}\right){ }^{5} \mathrm{H}+6 \% 2\left({ }^{5} \mathrm{D}^{4} \mathrm{D}\right){ }^{5} \mathrm{P}+6 \% 2\left({ }^{3} \mathrm{G}^{4} \mathrm{G}\right){ }^{5} \mathrm{G}$ $12 \% 1\left|\left({ }^{2} \mathrm{D} 1\right){ }^{3} \mathrm{~F}+8 \% 1\right|\left({ }^{2} \mathrm{D} 1\right){ }^{1} \mathrm{~F}+8 \% 1 \mid\left({ }^{2} \mathrm{D} 1\right){ }^{3} \mathrm{D}$ $51 \% 1\left|\left({ }^{2} \mathrm{P}\right){ }^{3} \mathrm{D}+5 \% 2\right|\left({ }^{3} \mathrm{H}^{4} \mathrm{H}\right){ }^{5} \mathrm{H}+5 \% 1 \mid\left({ }^{2} \mathrm{G} 1\right){ }^{1} \mathrm{~F}$ $10 \% 2\left|\left({ }^{5} \mathrm{D}^{4} \mathrm{D}\right){ }^{5} \mathrm{P}+10 \% 1\right|\left({ }^{2} \mathrm{P}\right){ }^{3} \mathrm{D}+9 \% 2 \mid\left({ }^{5} \mathrm{D}^{6} \mathrm{D}\right){ }^{5} \mathrm{P}$ $14 \% 22\left({ }^{5} \mathrm{D}^{4} \mathrm{D}\right){ }^{5} \mathrm{~F}+13 \% 2\left|\left({ }^{3} \mathrm{G}^{4} \mathrm{G}\right){ }^{5} \mathrm{H}+8 \% 2\right|\left({ }^{5} \mathrm{D}^{6} \mathrm{D}\right){ }^{5} \mathrm{~F}$ $24 \% 1\left|\left({ }^{2} \mathrm{G} 1\right){ }^{1} \mathrm{~F}+19 \% 1\right|\left({ }^{2} \mathrm{G} 1\right){ }^{3} \mathrm{G}+8 \% 1 \mid\left({ }^{2} \mathrm{~F} 2\right){ }^{1} \mathrm{~F}$ $22 \% 2\left({ }^{3} \mathrm{H}^{4} \mathrm{H}\right){ }^{5} \mathrm{H}+13 \% 2\left({ }^{5} \mathrm{D}^{4} \mathrm{D}\right){ }^{5} \mathrm{~F}+8 \% 2\left({ }^{5} \mathrm{D}^{6} \mathrm{D}\right){ }^{5} \mathrm{~F}$ $31 \% 1\left|\left({ }^{2} \mathrm{G} 1\right){ }^{3} \mathrm{~F}+21 \% 1\right|\left({ }^{2} \mathrm{G} 1\right){ }^{1} \mathrm{~F}+12 \% 1 \mid\left({ }^{2} \mathrm{D} 2\right){ }^{1} \mathrm{~F}$ $25 \% 2\left|\left({ }^{3} \mathrm{G}^{4} \mathrm{G}\right){ }^{5} \mathrm{H}+15 \% 2\right|\left({ }^{3} \mathrm{H}^{4} \mathrm{H}\right){ }^{5} \mathrm{H}+10 \% 2 \mid\left({ }^{5} \mathrm{D}^{6} \mathrm{D}\right){ }^{7} \mathrm{D}$ $13 \% 1\left|\left({ }^{2} \mathrm{D} 2\right){ }^{3} \mathrm{D}+11 \% 1\right|\left({ }^{2} \mathrm{G} 1\right){ }^{3} \mathrm{G}+10 \% 1 \mid\left({ }^{2} \mathrm{~F} 2\right){ }^{3} \mathrm{~F}$ $34 \% 2\left|\left({ }^{5} \mathrm{D}^{6} \mathrm{D}\right){ }^{7} \mathrm{D}+31 \% 2\right|\left({ }^{5} \mathrm{D}^{6} \mathrm{D}\right){ }^{7} \mathrm{P}+6 \% 2 \mid\left({ }^{5} \mathrm{D}^{6} \mathrm{D}\right){ }^{7} \mathrm{~F}$ $17 \% 1\left|\left({ }^{2} \mathrm{D} 2\right){ }^{1} \mathrm{~F}+15 \% 1\right|\left({ }^{2} \mathrm{G} 2\right){ }^{1} \mathrm{~F}+6 \% 1 \mid\left({ }^{2} \mathrm{~F} 1\right){ }^{1} \mathrm{~F}$ $22 \% 1\left|\left({ }^{2} \mathrm{D} 2\right){ }^{3} \mathrm{D}+13 \% 1\right|\left({ }^{2} \mathrm{D} 2\right){ }^{3} \mathrm{~F}+9 \% 1 \mid\left({ }^{4} \mathrm{~F}\right){ }^{3} \mathrm{D}$ $26 \% 1\left|\left({ }^{2} \mathrm{D} 2\right){ }^{3} \mathrm{~F}+14 \% 1\right|\left({ }^{2} \mathrm{G} 2\right){ }^{3} \mathrm{~F}+11 \% 1 \mid\left({ }^{2} \mathrm{H}\right){ }^{3} \mathrm{G}$ $12 \% 1\left|\left({ }^{2} \mathrm{~F} 1\right){ }^{1} \mathrm{~F}+11 \% 1\right|\left({ }^{2} \mathrm{~F} 2\right){ }^{1} \mathrm{~F}+8 \% 1 \mid\left({ }^{2} \mathrm{D} 3\right){ }^{1} \mathrm{~F}$ 


\begin{tabular}{crrrrrrr}
- & 169118.70 & - & & & & & \\
- & 168796.00 & - & & & & & \\
- & 167584.91 & \multicolumn{1}{c}{-} & & & & & \\
164689.65 & 164728.49 & -38.84 & 5 & 5 & 0.22 & 0.31 & 0.30 \\
163735.00 & 163748.28 & -13.28 & 8 & 3 & 0.34 & 0.30 & 0.40 \\
- & 163529.91 & - & & & & & \\
163205.61 & 163185.15 & 20.46 & 8 & 7 & 0.22 & 0.16 & 0.30 \\
162374.44 & 162374.33 & 0.11 & 8 & 5 & 0.23 & 0.30 & 0.30 \\
161368.69 & 161341.03 & 27.66 & 6 & 2 & 0.63 & 0.69 & 0.70 \\
159629.42 & 159664.68 & -35.26 & 6 & 3 & 0.33 & 0.54 & 0.40 \\
159189.79 & 159165.29 & 24.50 & 2 & 2 & 0.27 & 0.09 & 0.30 \\
- & 156974.70 & - & & & & & \\
156757.56 & 156711.63 & 45.93 & 11 & 9 & 0.15 & 0.10 & 0.17 \\
155085.02 & 155011.24 & 73.78 & 13 & 7 & 0.22 & 0.10 & 0.23 \\
154152.55 & 154184.96 & -32.41 & 13 & 9 & 0.18 & 0.13 & 0.20 \\
152373.63 & 152376.24 & -2.61 & 13 & 9 & 0.19 & 0.18 & 0.23 \\
151395.26 & 151433.97 & -38.71 & 11 & 5 & 0.14 & 0.09 & 0.16 \\
150235.09 & 150263.61 & -28.52 & 11 & 8 & 0.17 & 0.21 & 0.24 \\
148358.78 & 148333.66 & 25.12 & 8 & 7 & 0.19 & 0.13 & 0.20 \\
147341.74 & 147269.89 & 71.85 & 10 & 8 & 0.17 & 0.10 & 0.20 \\
146186.18 & 146243.51 & -57.33 & 17 & 12 & 0.14 & 0.16 & 0.22 \\
145536.69 & 145547.22 & -10.53 & 7 & 7 & 0.16 & 0.23 & 0.30 \\
144855.15 & 144824.43 & 30.72 & 15 & 9 & 0.11 & 0.11 & 0.14 \\
141214.36 & 141218.96 & -4.60 & 14 & 13 & 0.14 & 0.14 & 0.20 \\
140526.92 & 140527.31 & -0.39 & 15 & 12 & 0.12 & 0.13 & 0.17 \\
140054.55 & 140017.45 & 37.10 & 11 & 8 & 0.09 & 0.07 & 0.12 \\
138529.81 & 138518.02 & 11.79 & 10 & 9 & 0.12 & 0.12 & 0.16 \\
137700.64 & 137686.28 & 14.36 & 12 & 10 & 0.13 & 0.11 & 0.17 \\
135420.03 & 135410.98 & 9.05 & 15 & 10 & 0.11 & 0.11 & 0.14 \\
133037.83 & 133034.39 & 3.44 & 14 & 13 & 0.09 & 0.05 & 0.12 \\
129741.29 & 129737.88 & 3.41 & 10 & 8 & 0.08 & 0.08 & 0.09 \\
129069.98 & 129131.00 & -61.02 & 10 & 10 & 0.09 & 0.08 & 0.11 \\
123202.06 & 123208.94 & -6.88 & 13 & 11 & 0.08 & 0.06 & 0.10 \\
121902.29 & 121893.49 & 8.80 & 14 & 11 & 0.09 & 0.09 & 0.10 \\
118330.45 & 118327.97 & 2.48 & 13 & 12 & 0.07 & 0.06 & 0.10 \\
115636.19 & 115643.34 & -7.15 & 8 & 7 & 0.10 & 0.09 & 0.13 \\
100573.47 & 100535.79 & 37.68 & 4 & 4 & 0.14 & 0.15 & 0.17
\end{tabular}

$\mathrm{J}=4$

198291.74

197472.34 -

$197186.72 \quad-$

195583.15

195317.45

191935.47

191481.18

187844.45

185979.06

184419.33

182444.86

180629.97

180103.44

177363.27

177330.91

175027.06

$\begin{array}{lllllll}174985.31 & 41.75 & 4 & 4 & 0.22 & 0.05 & 0.24\end{array}$
$46 \% 2\left|\left({ }^{5} \mathrm{D}^{6} \mathrm{D}\right){ }^{7} \mathrm{P}+28 \% 2\right|\left({ }^{5} \mathrm{D}^{6} \mathrm{D}\right){ }^{7} \mathrm{D}+7 \% 2 \mid\left({ }^{5} \mathrm{D}^{6} \mathrm{D}\right){ }^{5} \mathrm{P}$ $26 \% 1\left|\left({ }^{2} \mathrm{G} 1\right){ }^{3} \mathrm{G}+9 \% 1\right|\left({ }^{2} \mathrm{G} 1\right){ }^{1} \mathrm{~F}+8 \% 1 \mid\left({ }^{4} \mathrm{~F}\right){ }^{3} \mathrm{~F}$ $15 \% 1\left|\left({ }^{2} \mathrm{~F} 2\right){ }^{1} \mathrm{~F}+14 \% 1\right|\left({ }^{2} \mathrm{~F} 1\right){ }^{1} \mathrm{~F}+12 \% 1 \mid\left({ }^{2} \mathrm{G} 1\right){ }^{3} \mathrm{~F}$ $27 \% 1\left|\left({ }^{4} \mathrm{~F}\right) \quad{ }^{3} \mathrm{G}+9 \% 1\right|\left({ }^{2} \mathrm{D} 3\right){ }^{3} \mathrm{D}+8 \% 1 \mid\left({ }^{2} \mathrm{~F} 1\right){ }^{3} \mathrm{D}$ $16 \% 2\left|\left({ }^{5} \mathrm{D}^{6} \mathrm{D}\right){ }^{7} \mathrm{~F}+14 \% 1\right|\left({ }^{2} \mathrm{~F} 2\right){ }^{1} \mathrm{~F}+10 \% 1 \mid\left({ }^{2} \mathrm{~F} 2\right){ }^{3} \mathrm{~F}$ $53 \% 2\left|\left({ }^{5} \mathrm{D}^{6} \mathrm{D}\right){ }^{7} \mathrm{~F}+5 \% 1\right|\left({ }^{2} \mathrm{~F} 2\right){ }^{1} \mathrm{~F}+4 \% 2 \mid\left({ }^{5} \mathrm{D}^{6} \mathrm{D}\right){ }^{7} \mathrm{D}$ $34 \% 1\left|\left({ }^{2} \mathrm{H}\right){ }^{3} \mathrm{G}+12 \% 1\right|\left({ }^{4} \mathrm{~F}\right){ }^{3} \mathrm{D}+8 \% 2 \mid\left({ }^{5} \mathrm{D}^{6} \mathrm{D}\right){ }^{7} \mathrm{~F}$ $20 \% 1\left|\left({ }^{2} \mathrm{G} 2\right){ }^{3} \mathrm{G}+13 \% 1\right|\left({ }^{2} \mathrm{~F} 2\right){ }^{3} \mathrm{D}+12 \% 1 \mid\left({ }^{2} \mathrm{G} 2\right){ }^{1} \mathrm{~F}$ $16 \% 1\left|\left({ }^{2} \mathrm{D} 3\right){ }^{3} \mathrm{~F}+14 \% 1\right|\left({ }^{2} \mathrm{D} 2\right){ }^{3} \mathrm{D}+14 \% 1 \mid\left({ }^{2} \mathrm{~F} 2\right){ }^{3} \mathrm{G}$ $19 \% 1\left|\left({ }^{2} \mathrm{~F} 1\right){ }^{3} \mathrm{~F}+11 \% 1\right|\left({ }^{2} \mathrm{D} 2\right){ }^{3} \mathrm{~F}+8 \% 1 \mid\left({ }^{4} \mathrm{P}\right){ }^{3} \mathrm{D}$ $20 \% 1\left|\left({ }^{2} \mathrm{~F} 1\right){ }^{3} \mathrm{G}+15 \% 1\right|\left({ }^{4} \mathrm{~F}\right){ }^{5} \mathrm{~F}+9 \% 1 \mid\left({ }^{2} \mathrm{D} 3\right){ }^{3} \mathrm{~F}$ $27 \% 1\left|\left({ }^{2} \mathrm{G} 2\right){ }^{3} \mathrm{~F}+10 \% 1\right|\left({ }^{4} \mathrm{~F}\right) \quad{ }^{3} \mathrm{D}+8 \% 1 \mid\left({ }^{2} \mathrm{H}\right){ }^{3} \mathrm{G}$ $26 \% 1\left|\left({ }^{4} \mathrm{~F}\right) \quad{ }^{3} \mathrm{~F}+13 \% 1\right|\left({ }^{2} \mathrm{G} 2\right){ }^{1} \mathrm{~F}+10 \% 1 \mid\left({ }^{2} \mathrm{~F} 2\right){ }^{1} \mathrm{~F}$ $16 \% 1\left|\left({ }^{4} \mathrm{~F}\right) \quad{ }^{3} \mathrm{G}+10 \% 1\right|\left({ }^{2} \mathrm{~F} 1\right){ }^{3} \mathrm{D}+7 \% 1 \mid\left({ }^{2} \mathrm{~F} 2\right){ }^{1} \mathrm{~F}$ $12 \% 1\left|\left({ }^{2} \mathrm{D} 3\right){ }^{3} \mathrm{D}+10 \% 1\right|\left({ }^{4} \mathrm{P}\right){ }^{5} \mathrm{P}+8 \% 1 \mid\left({ }^{2} \mathrm{~F} 1\right){ }^{3} \mathrm{D}$ $19 \% 1\left|\left({ }^{2} \mathrm{~F} 2\right) \quad{ }^{3} \mathrm{~F}+11 \% 1\right|\left({ }^{4} \mathrm{P}\right) \quad{ }^{3} \mathrm{D}+10 \% 1 \mid\left({ }^{4} \mathrm{~F}\right) \quad{ }^{5} \mathrm{D}$ $30 \% 1\left|\left({ }^{4} \mathrm{~F}\right) \quad{ }^{5} \mathrm{~F}+16 \% 1\right|\left({ }^{2} \mathrm{~F} 1\right){ }^{3} \mathrm{D}+5 \% 1 \mid\left({ }^{2} \mathrm{~F} 2\right){ }^{3} \mathrm{D}$ $14 \% 1\left|\left({ }^{4} \mathrm{G}\right) \quad{ }^{3} \mathrm{G}+14 \% 1\right|\left({ }^{4} \mathrm{D}\right) \quad{ }^{3} \mathrm{D}+7 \% 1 \mid\left({ }^{2} \mathrm{D} 3\right){ }^{3} \mathrm{~F}$ $10 \% 1\left|\left({ }^{2} \mathrm{~F} 2\right){ }^{3} \mathrm{D}+8 \% 1\right|\left({ }^{4} \mathrm{~F}\right) \quad{ }^{5} \mathrm{G}+8 \% 1 \mid\left({ }^{4} \mathrm{D}\right){ }^{3} \mathrm{~F}$ $15 \% 1\left|\left({ }^{2} \mathrm{G} 2\right) \quad{ }^{3} \mathrm{G}+13 \% 1\right|\left({ }^{4} \mathrm{P}\right) \quad{ }^{5} \mathrm{P}+12 \% 1 \mid\left({ }^{4} \mathrm{D}\right) \quad{ }^{5} \mathrm{D}$ $15 \% 1\left|\left({ }^{2} \mathrm{G} 2\right) \quad{ }^{3} \mathrm{G}+11 \% 1\right|\left({ }^{4} \mathrm{~F}\right) \quad{ }^{5} \mathrm{G}+9 \% 1 \mid\left({ }^{4} \mathrm{G}\right) \quad{ }^{3} \mathrm{G}$ $12 \% 1\left|\left({ }^{4} \mathrm{P}\right) \quad{ }^{5} \mathrm{D}+12 \% 1\right|\left({ }^{2} \mathrm{~F} 2\right){ }^{3} \mathrm{G}+9 \% 1 \mid\left({ }^{4} \mathrm{D}\right){ }^{5} \mathrm{~F}$ $19 \% 1\left|\left({ }^{4} \mathrm{G}\right) \quad{ }^{3} \mathrm{~F}+12 \% 1\right|\left({ }^{4} \mathrm{D}\right) \quad{ }^{3} \mathrm{D}+8 \% 1 \mid\left({ }^{4} \mathrm{G}\right){ }^{5} \mathrm{~F}$ $20 \% 1\left|\left({ }^{4} \mathrm{G}\right) \quad{ }^{5} \mathrm{~F}+19 \% 1\right|\left({ }^{4} \mathrm{G}\right) \quad{ }^{3} \mathrm{G}+10 \% 1 \mid\left({ }^{2} \mathrm{~F} 1\right) \quad{ }^{1} \mathrm{~F}$ $16 \% 1\left|\left({ }^{4} \mathrm{D}\right) \quad{ }^{3} \mathrm{~F}+16 \% 1\right|\left({ }^{4} \mathrm{~F}\right) \quad{ }^{5} \mathrm{G}+9 \% 1 \mid\left({ }^{2} \mathrm{~F} 1\right){ }^{3} \mathrm{~F}$ $9 \% 1\left|\left({ }^{4} \mathrm{G}\right){ }^{3} \mathrm{~F}+9 \% 1\right|\left({ }^{2} \mathrm{~F} 2\right){ }^{3} \mathrm{G}+8 \% 1 \mid\left({ }^{4} \mathrm{G}\right){ }^{5} \mathrm{G}$ $19 \% 1\left|\left({ }^{4} \mathrm{P}\right) \quad{ }^{5} \mathrm{D}+14 \% 1\right|\left({ }^{4} \mathrm{D}\right) \quad{ }^{5} \mathrm{~F}+13 \% 1 \mid\left({ }^{4} \mathrm{D}\right) \quad{ }^{5} \mathrm{D}$ $17 \% 1\left|\left({ }^{4} \mathrm{G}\right) \quad{ }^{3} \mathrm{~F}+11 \% 1\right|\left({ }^{4} \mathrm{G}\right) \quad{ }^{5} \mathrm{G}+8 \% 1 \mid\left({ }^{4} \mathrm{~F}\right) \quad{ }^{5} \mathrm{G}$ $17 \% 1\left|\left({ }^{4} \mathrm{D}\right) \quad{ }^{5} \mathrm{D}+9 \% 1\right|\left({ }^{2} \mathrm{~F} 1\right){ }^{3} \mathrm{D}+8 \% 1 \mid\left({ }^{4} \mathrm{G}\right){ }^{5} \mathrm{H}$ $20 \% 1\left|\left({ }^{4} \mathrm{G}\right) \quad{ }^{5} \mathrm{~F}+9 \% 1\right|\left({ }^{4} \mathrm{D}\right) \quad{ }^{5} \mathrm{P}+8 \% 1 \mid\left({ }^{4} \mathrm{~F}\right){ }^{5} \mathrm{~F}$ $9 \% 1\left|\left({ }^{4} \mathrm{D}\right) \quad{ }^{5} \mathrm{~F}+9 \% 1\right|\left({ }^{4} \mathrm{G}\right){ }^{5} \mathrm{H}+8 \% 1 \mid\left({ }^{2} \mathrm{D} 3\right){ }^{3} \mathrm{D}$ $30 \% 1\left|\left({ }^{4} \mathrm{D}\right) \quad{ }^{5} \mathrm{P}+15 \% 1\right|\left({ }^{4} \mathrm{G}\right) \quad{ }^{5} \mathrm{G}+11 \% 1 \mid\left({ }^{4} \mathrm{D}\right) \quad{ }^{3} \mathrm{D}$ $31 \% 1\left|\left({ }^{4} \mathrm{G}\right) \quad{ }^{5} \mathrm{H}+21 \% 1\right|\left({ }^{4} \mathrm{D}\right) \quad{ }^{5} \mathrm{~F}+9 \% 1 \mid\left({ }^{4} \mathrm{D}\right){ }^{5} \mathrm{D}$ $25 \% 1\left|\left({ }^{4} \mathrm{G}\right) \quad{ }^{5} \mathrm{G}+21 \% 1\right|\left({ }^{6} \mathrm{~S}\right) \quad{ }^{5} \mathrm{P}+16 \% 1 \mid\left({ }^{4} \mathrm{G}\right) \quad{ }^{3} \mathrm{~F}$ $29 \% 1\left|\left({ }^{6} \mathrm{~S}\right) \quad{ }^{5} \mathrm{P}+15 \% 1\right|\left({ }^{6} \mathrm{~S}\right) \quad{ }^{7} \mathrm{P}+10 \% 1 \mid\left({ }^{4} \mathrm{G}\right) \quad{ }^{5} \mathrm{G}$ $19 \% 1\left|\left({ }^{6} \mathrm{~S}\right) \quad{ }^{7} \mathrm{P}+15 \% 1\right|\left({ }^{4} \mathrm{G}\right) \quad{ }^{5} \mathrm{H}+11 \% 1 \mid\left({ }^{2} \mathrm{~F} 1\right){ }^{3} \mathrm{G}$ $53 \% 1\left|\left({ }^{6} \mathrm{~S}\right) \quad{ }^{7} \mathrm{P}+24 \% 1\right|\left({ }^{6} \mathrm{~S}\right) \quad{ }^{5} \mathrm{P}+6 \% 1 \mid\left({ }^{4} \mathrm{P}\right){ }^{5} \mathrm{P}$

$55 \% 1\left|\left({ }^{2} \mathrm{D} 1\right){ }^{3} \mathrm{~F}+10 \% 1\right|\left({ }^{2} \mathrm{D} 3\right){ }^{3} \mathrm{~F}+4 \% 1 \mid\left({ }^{2} \mathrm{G} 1\right){ }^{3} \mathrm{~F}$ $11 \% 2\left|\left({ }^{3} \mathrm{~F} 2{ }^{4} \mathrm{~F}\right){ }^{5} \mathrm{~F}+10 \% 1\right|\left({ }^{2} \mathrm{D} 1\right){ }^{3} \mathrm{~F}+9 \% 2 \mid\left({ }^{3} \mathrm{~F} 2{ }^{4} \mathrm{~F}\right){ }^{5} \mathrm{G}$ $24 \% 2\left|\left({ }^{3} \mathrm{G}^{4} \mathrm{G}\right){ }^{5} \mathrm{G}+12 \% 2\left({ }^{3} \mathrm{G}^{4} \mathrm{G}\right){ }^{5} \mathrm{H}+8 \% 2\right|\left({ }^{3} \mathrm{G}^{4} \mathrm{G}\right){ }^{5} \mathrm{~F}$ $9 \% 2\left|\left({ }^{3} \mathrm{G}^{2} \mathrm{G}\right){ }^{3} \mathrm{H}+8 \% 2\right|\left({ }^{3} \mathrm{G}^{4} \mathrm{G}\right){ }^{5} \mathrm{H}+7 \% 2 \mid\left({ }^{5} \mathrm{D}^{6} \mathrm{D}\right){ }^{5} \mathrm{D}$ $14 \% 2\left|\left({ }^{3} \mathrm{~F} 2{ }^{4} \mathrm{~F}\right){ }^{5} \mathrm{G}+8 \% 2\right|\left({ }^{3} \mathrm{D}^{4} \mathrm{D}\right){ }^{5} \mathrm{~F}+8 \% 2 \mid\left({ }^{3} \mathrm{D}^{4} \mathrm{D}\right){ }^{5} \mathrm{D}$ $16 \% 2\left|\left({ }^{5} \mathrm{D}^{6} \mathrm{D}\right){ }^{5} \mathrm{~F}+14 \% 2\right|\left({ }^{3} \mathrm{H}^{4} \mathrm{H}\right){ }^{5} \mathrm{H}+10 \% 2 \mid\left({ }^{3} \mathrm{G}^{4} \mathrm{G}\right){ }^{5} \mathrm{~F}$ $14 \% 2$ 2 $\left({ }^{3} \mathrm{H}^{4} \mathrm{H}\right){ }^{5} \mathrm{H}+11 \% 2\left|\left({ }^{3} \mathrm{~F} 2^{4} \mathrm{~F}\right){ }^{5} \mathrm{D}+6 \% 2\right|\left({ }^{5} \mathrm{D}^{4} \mathrm{D}\right){ }^{3} \mathrm{~F}$ $20 \% 2\left|\left({ }^{3} \mathrm{G}^{4} \mathrm{G}\right){ }^{5} \mathrm{H}+7 \% 2\right|\left({ }^{5} \mathrm{D}^{4} \mathrm{D}\right){ }^{3} \mathrm{~F}+6 \% 2 \mid\left({ }^{5} \mathrm{D}^{6} \mathrm{D}\right){ }^{7} \mathrm{P}$ $33 \% 2\left|\left({ }^{3} \mathrm{H}^{4} \mathrm{H}\right){ }^{5} \mathrm{I}+9 \% 2\right|\left({ }^{5} \mathrm{D}^{6} \mathrm{D}\right){ }^{7} \mathrm{D}+8 \% 2 \mid\left({ }^{3} \mathrm{~F} 2{ }^{4} \mathrm{~F}\right){ }^{5} \mathrm{G}$ $19 \% 2\left|\left({ }^{3} \mathrm{H}^{4} \mathrm{H}\right){ }^{5} \mathrm{H}+7 \% 2\left({ }^{3} \mathrm{G}^{4} \mathrm{G}\right){ }^{5} \mathrm{~F}+7 \% 2\right|\left({ }^{3} \mathrm{H}^{4} \mathrm{H}\right){ }^{5} \mathrm{G}$ $58 \% 1\left|\left({ }^{2} \mathrm{G} 1\right){ }^{1} \mathrm{G}+6 \% 1\right|\left({ }^{2} \mathrm{G} 1\right){ }^{3} \mathrm{H}+5 \% 1 \mid\left({ }^{2} \mathrm{G} 1\right){ }^{3} \mathrm{~F}$ $53 \% 2\left|\left({ }^{5} \mathrm{D}^{6} \mathrm{D}\right){ }^{7} \mathrm{D}+14 \% 2\right|\left({ }^{5} \mathrm{D}^{6} \mathrm{D}\right){ }^{7} \mathrm{~F}+9 \% 2 \mid\left({ }^{5} \mathrm{D}^{6} \mathrm{D}\right){ }^{7} \mathrm{P}$ $53 \% 1\left|\left({ }^{2} \mathrm{G} 1\right){ }^{3} \mathrm{G}+12 \% 1\right|\left({ }^{2} \mathrm{G} 1\right){ }^{3} \mathrm{~F}+10 \% 1 \mid\left({ }^{2} \mathrm{G} 1\right){ }^{3} \mathrm{H}$ $25 \% 1\left|\left({ }^{2} \mathrm{D} 2\right){ }^{3} \mathrm{~F}+13 \% 2\right|\left({ }^{3} \mathrm{H}^{4} \mathrm{H}\right){ }^{5} \mathrm{I}+8 \% 2 \mid\left({ }^{3} \mathrm{G}^{4} \mathrm{G}\right){ }^{5} \mathrm{H}$ $22 \% 1\left|\left({ }^{2} \mathrm{D} 2\right){ }^{3} \mathrm{~F}+15 \% 2\right|\left({ }^{3} \mathrm{H}^{4} \mathrm{H}\right){ }^{5} \mathrm{I}+8 \% 2 \mid\left({ }^{3} \mathrm{G}^{4} \mathrm{G}\right){ }^{5} \mathrm{H}$ $21 \% 1\left|\left({ }^{2} \mathrm{H}\right) \quad{ }^{1} \mathrm{G}+11 \% 1\right|\left({ }^{2} \mathrm{H}\right){ }^{3} \mathrm{G}+10 \% 1 \mid\left({ }^{2} \mathrm{I}\right){ }^{3} \mathrm{H}$ $11 \% 1\left|\left({ }^{2} \mathrm{D} 2\right){ }^{3} \mathrm{~F}+11 \% 1\right|\left({ }^{2} \mathrm{D} 3\right){ }^{3} \mathrm{~F}+9 \% 1 \mid\left({ }^{4} \mathrm{~F}\right){ }^{3} \mathrm{~F}$ 


\begin{tabular}{|c|c|c|c|c|c|c|c|}
\hline 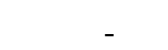 & 170927.43 & - & & & & & \\
\hline - & 170854.27 & - & & & & & \\
\hline - & 167520.14 & - & & & & & \\
\hline 167107.33 & 167063.13 & 44.20 & 7 & 7 & 0.18 & 0.22 & 0.20 \\
\hline 166876.33 & 166813.62 & 62.71 & 3 & 3 & 0.34 & 0.52 & 0.50 \\
\hline 164743.73 & 164748.80 & -5.07 & 8 & 6 & 0.22 & 0.31 & 0.30 \\
\hline 163997.14 & 164033.61 & -36.47 & 8 & 6 & 0.19 & 0.17 & 0.21 \\
\hline 161902.01 & 161926.09 & -24.08 & 9 & 7 & 0.18 & 0.26 & 0.30 \\
\hline 160207.91 & 160183.54 & 24.37 & 7 & 7 & 0.17 & 0.13 & 0.22 \\
\hline 159791.70 & 159777.99 & 13.71 & 8 & 4 & 0.31 & 0.31 & 0.40 \\
\hline 158188.33 & 158172.92 & 15.41 & 12 & 10 & 0.15 & 0.08 & 0.16 \\
\hline 157167.25 & 157183.69 & -16.44 & 11 & 7 & 0.21 & 0.09 & 0.22 \\
\hline 155597.91 & 1556 & -34.10 & 10 & 10 & 0.15 & 0.11 & 0.20 \\
\hline 153828.48 & 1538 & -46.32 & 12 & 11 & 0.15 & 0.20 & 0.30 \\
\hline 153412.13 & 153404.99 & 7.14 & 14 & 9 & 0.13 & 0.09 & 0.16 \\
\hline 152982.27 & 152980.29 & 1.98 & 8 & 8 & 0.15 & 0.09 & 0.18 \\
\hline 150706.05 & 150717.71 & -11.66 & 11 & 7 & 0.13 & 0.13 & 0.19 \\
\hline 147884.68 & 147919.32 & -34.64 & 11 & 9 & 0.15 & 0.15 & 0.17 \\
\hline 147763.22 & 147762.13 & 1.09 & 11 & 11 & 0.14 & 0.14 & 0.19 \\
\hline 145439.95 & 145458.47 & -18.52 & 16 & 10 & 0.10 & 0.08 & 0.12 \\
\hline 145003.78 & 14496 & 38.60 & 19 & 16 & 0.09 & 0.06 & 0.11 \\
\hline 143156.95 & 143163.67 & -6.72 & 16 & 9 & 0.08 & 0.05 & 0.11 \\
\hline 142198.78 & 142168.70 & 30.08 & 13 & 10 & 0.10 & 0.06 & 0.12 \\
\hline 141141.16 & 141171.31 & -30.15 & 15 & 13 & 0.12 & 0.11 & 0.15 \\
\hline 139998.38 & 140001.58 & -3.20 & 15 & 13 & 0.10 & 0.09 & 0.12 \\
\hline 137277.36 & 137245.26 & 32.10 & 9 & 8 & 0.13 & 0.08 & 0.14 \\
\hline 135595.44 & 135565.34 & 30.10 & 17 & 12 & 0.10 & 0.07 & 0.11 \\
\hline 134025.31 & 134014.35 & 10.96 & 16 & 12 & 0.08 & 0.06 & 0.10 \\
\hline 132088.18 & 132082.67 & 5.51 & 15 & 11 & 0.09 & 0.10 & 0.12 \\
\hline 128493.21 & 128473.88 & 19.33 & 14 & 11 & 0.07 & 0.06 & 0.09 \\
\hline 125224.81 & 125156.82 & 67.99 & 15 & 13 & 0.07 & 0.06 & 0.09 \\
\hline 120722.59 & 120733.78 & -11.19 & 10 & 6 & 0.09 & 0.10 & 0.13 \\
\hline 113462.35 & 113485.10 & -22.75 & 4 & 4 & 0.21 & 0.23 & 0.30 \\
\hline
\end{tabular}

$\mathrm{J}=5$
183164.92

182764.37

$181326.21-$

181182.28

$171674.89-$

$\begin{array}{llrrrrrr}170618.53 & 170636.68 & -18.15 & 6 & 6 & 0.19 & 0.14 & 0.23 \\ 169490.49 & 169473.02 & 17.47 & 8 & 5 & 0.18 & 0.11 & 0.20 \\ 166282.72 & 166258.86 & 23.86 & 5 & 4 & 0.32 & 0.04 & 0.30 \\ 165239.60 & 165294.83 & -55.23 & 6 & 6 & 0.18 & 0.18 & 0.24 \\ 163240.75 & 163235.56 & 5.19 & 7 & 5 & 0.19 & 0.26 & 0.30 \\ 162653.94 & 162690.06 & -36.12 & 11 & 11 & 0.14 & 0.14 & 0.20 \\ 159762.71 & 159781.25 & -18.54 & 8 & 5 & 0.23 & 0.26 & 0.30 \\ 157481.05 & 157491.21 & -10.16 & 12 & 11 & 0.13 & 0.09 & 0.16 \\ 155160.61 & 155168.40 & -7.79 & 12 & 9 & 0.14 & 0.09 & 0.16 \\ 153658.98 & 153658.98 & 0.00 & 12 & 10 & 0.13 & 0.11 & 0.17 \\ 152304.45 & 152289.59 & 14.86 & 15 & 13 & 0.11 & 0.08 & 0.13 \\ 151000.14 & 151024.33 & -24.19 & 10 & 8 & 0.16 & 0.19 & 0.30 \\ 148937.72 & 148952.96 & -15.24 & 12 & 9 & 0.17 & 0.16 & 0.22 \\ 145525.25 & 145492.13 & 33.12 & 17 & 16 & 0.11 & 0.08 & 0.13 \\ 144512.45 & 144496.17 & 16.28 & 14 & 10 & 0.09 & 0.07 & 0.12 \\ 143731.85 & 143726.16 & 5.69 & 14 & 10 & 0.12 & 0.06 & 0.13\end{array}$
$46 \% 2\left|\left({ }^{5} \mathrm{D}^{6} \mathrm{D}\right){ }^{7} \mathrm{P}+6 \% 2\right|\left({ }^{5} \mathrm{D}^{6} \mathrm{D}\right){ }^{7} \mathrm{~F}+5 \% 2 \mid\left({ }^{5} \mathrm{D}^{6} \mathrm{D}\right){ }^{7} \mathrm{D}$ $15 \% 2\left|\left({ }^{5} \mathrm{D}^{6} \mathrm{D}\right){ }^{7} \mathrm{P}+7 \% 1\right|\left({ }^{2} \mathrm{G} 1\right){ }^{3} \mathrm{~F}+6 \% 1 \mid\left({ }^{2} \mathrm{D} 3\right){ }^{3} \mathrm{~F}$ $39 \% 2\left|\left({ }^{5} \mathrm{D}^{6} \mathrm{D}\right){ }^{7} \mathrm{~F}+7 \% 2\right|\left({ }^{5} \mathrm{D}^{6} \mathrm{D}\right){ }^{7} \mathrm{D}+6 \% 1 \mid\left({ }^{4} \mathrm{~F}\right){ }^{5} \mathrm{~F}$ $12 \% 1\left|\left({ }^{2} \mathrm{~F} 2\right){ }^{1} \mathrm{G}+10 \% 1\right|\left({ }^{2} \mathrm{H}\right){ }^{1} \mathrm{G}+9 \% 1 \mid\left({ }^{4} \mathrm{~F}\right){ }^{3} \mathrm{G}$ $17 \% 1\left|\left({ }^{2} \mathrm{G} 1\right){ }^{3} \mathrm{H}+14 \% 2\right|\left({ }^{5} \mathrm{D}^{6} \mathrm{D}\right){ }^{7} \mathrm{~F}+8 \% 1 \mid\left({ }^{2} \mathrm{~F} 2\right){ }^{3} \mathrm{~F}$ $17 \% 1\left|\left({ }^{2} \mathrm{G} 1\right){ }^{3} \mathrm{~F}+13 \% 1\right|\left({ }^{2} \mathrm{H}\right){ }^{3} \mathrm{G}+10 \% 1 \mid\left({ }^{2} \mathrm{G} 1\right){ }^{1} \mathrm{G}$ $16 \% 1\left|\left({ }^{2} \mathrm{G} 1\right){ }^{3} \mathrm{~F}+11 \% 1\right|\left({ }^{2} \mathrm{~F} 2\right){ }^{1} \mathrm{G}+10 \% 1 \mid\left({ }^{2} \mathrm{G} 2\right){ }^{1} \mathrm{G}$ $16 \% 1\left|\left({ }^{2} \mathrm{H}\right){ }^{3} \mathrm{H}+15 \% 1\right|\left({ }^{2} \mathrm{G} 2\right){ }^{1} \mathrm{G}+13 \% 1 \mid\left({ }^{2} \mathrm{G} 2\right){ }^{3} \mathrm{G}$ $20 \% 1\left|\left({ }^{4} \mathrm{~F}\right) \quad{ }^{3} \mathrm{~F}+19 \% 1\right|\left({ }^{2} \mathrm{~F} 2\right){ }^{3} \mathrm{G}+5 \% 1 \mid\left({ }^{4} \mathrm{G}\right){ }^{3} \mathrm{G}$ $14 \% 1\left|\left({ }^{2} \mathrm{~F} 1\right) \quad{ }^{3} \mathrm{~F}+10 \% 1\right|\left({ }^{4} \mathrm{~F}\right) \quad{ }^{3} \mathrm{G}+10 \% 1 \mid\left({ }^{2} \mathrm{~F} 1\right){ }^{1} \mathrm{G}$ $12 \% 1\left|\left({ }^{2} \mathrm{~F} 1\right) \quad{ }^{3} \mathrm{G}+11 \% 1\right|\left({ }^{4} \mathrm{~F}\right) \quad{ }^{5} \mathrm{G}+10 \% 1 \mid\left({ }^{2} \mathrm{~F} 2\right){ }^{3} \mathrm{~F}$ $15 \% 1\left|\left({ }^{4} \mathrm{P}\right) \quad{ }^{5} \mathrm{D}+12 \% 1\right|\left({ }^{2} \mathrm{~F} 1\right){ }^{1} \mathrm{G}+10 \% 1 \mid\left({ }^{2} \mathrm{D} 3\right){ }^{3} \mathrm{~F}$ $17 \% 1\left|\left({ }^{2} \mathrm{G} 2\right){ }^{3} \mathrm{~F}+15 \% 1\right|\left({ }^{2} \mathrm{H}\right){ }^{3} \mathrm{H}+8 \% 1 \mid\left({ }^{2} \mathrm{G} 2\right){ }^{3} \mathrm{G}$ $33 \% 1\left|\left({ }^{2} \mathrm{I}\right) \quad{ }^{3} \mathrm{H}+12 \% 1\right|\left({ }^{4} \mathrm{G}\right) \quad{ }^{3} \mathrm{~F}+10 \% 1 \mid\left({ }^{2} \mathrm{H}\right) \quad{ }^{3} \mathrm{G}$ $24 \% 1\left|\left({ }^{2} \mathrm{~F} 2\right){ }^{3} \mathrm{~F}+10 \% 1\right|\left({ }^{4} \mathrm{~F}\right) \quad{ }^{5} \mathrm{G}+9 \% 1 \mid\left({ }^{2} \mathrm{~F} 1\right){ }^{3} \mathrm{~F}$ $18 \% 1\left|\left({ }^{2} \mathrm{G} 2\right){ }^{3} \mathrm{G}+10 \% 1\right|\left({ }^{2} \mathrm{G} 2\right){ }^{1} \mathrm{G}+7 \% 1 \mid\left({ }^{2} \mathrm{I}\right){ }^{3} \mathrm{H}$ $17 \% 1\left|\left({ }^{4} \mathrm{~F}\right) \quad{ }^{5} \mathrm{G}+16 \% 1\right|\left({ }^{2} \mathrm{~F} 1\right){ }^{3} \mathrm{G}+11 \% 1 \mid\left({ }^{2} \mathrm{G} 2\right){ }^{3} \mathrm{~F}$ $15 \% 1\left|\left({ }^{4} \mathrm{D}\right) \quad{ }^{3} \mathrm{~F}+14 \% 1\right|\left({ }^{2} \mathrm{I}\right) \quad{ }^{3} \mathrm{H}+8 \% 1 \mid\left({ }^{2} \mathrm{H}\right) \quad{ }^{3} \mathrm{G}$ $11 \% 1\left|\left({ }^{2} \mathrm{H}\right) \quad{ }^{1} \mathrm{G}+11 \% 1\right|\left({ }^{4} \mathrm{G}\right) \quad{ }^{3} \mathrm{G}+11 \% 1 \mid\left({ }^{4} \mathrm{D}\right) \quad{ }^{3} \mathrm{~F}$ $18 \% 1\left|\left({ }^{4} \mathrm{~F}\right) \quad{ }^{5} \mathrm{~F}+15 \% 1\right|\left({ }^{4} \mathrm{D}\right) \quad{ }^{5} \mathrm{D}+8 \% 1 \mid\left({ }^{4} \mathrm{G}\right) \quad{ }^{5} \mathrm{~F}$ $31 \% 1\left|\left({ }^{4} \mathrm{G}\right) \quad{ }^{3} \mathrm{G}+12 \% 1\right|\left({ }^{2} \mathrm{~F} 1\right) \quad{ }^{3} \mathrm{~F}+7 \% 1 \mid\left({ }^{4} \mathrm{G}\right){ }^{5} \mathrm{H}$ $26 \% 1\left|\left({ }^{2} \mathrm{G} 2\right){ }^{3} \mathrm{H}+14 \% 1\right|\left({ }^{2} \mathrm{~F} 2\right){ }^{3} \mathrm{G}+10 \% 1 \mid\left({ }^{2} \mathrm{G} 2\right){ }^{1} \mathrm{G}$ $16 \% 1\left|\left({ }^{2} \mathrm{H}\right) \quad{ }^{3} \mathrm{H}+11 \% 1\right|\left({ }^{4} \mathrm{D}\right) \quad{ }^{5} \mathrm{D}+11 \% 1 \mid\left({ }^{2} \mathrm{G} 2\right){ }^{3} \mathrm{H}$ $39 \% 1\left|\left({ }^{4} \mathrm{G}\right) \quad{ }^{3} \mathrm{~F}+16 \% 1\right|\left({ }^{4} \mathrm{G}\right) \quad{ }^{5} \mathrm{G}+7 \% 1 \mid\left({ }^{4} \mathrm{D}\right){ }^{5} \mathrm{D}$ $40 \% 1\left|\left({ }^{4} \mathrm{G}\right) \quad{ }^{3} \mathrm{H}+17 \% 1\right|\left({ }^{4} \mathrm{P}\right) \quad{ }^{5} \mathrm{D}+7 \% 1 \mid\left({ }^{4} \mathrm{D}\right){ }^{5} \mathrm{~F}$ $35 \% 1\left|\left({ }^{4} \mathrm{G}\right) \quad{ }^{5} \mathrm{~F}+12 \% 1\right|\left({ }^{4} \mathrm{D}\right) \quad{ }^{3} \mathrm{~F}+10 \% 1 \mid\left({ }^{4} \mathrm{~F}\right) \quad{ }^{5} \mathrm{~F}$ $25 \% 1\left|\left({ }^{4} \mathrm{G}\right) \quad{ }^{5} \mathrm{G}+11 \% 1\right|\left({ }^{4} \mathrm{D}\right) \quad{ }^{5} \mathrm{D}+11 \% 1 \mid\left({ }^{2} \mathrm{G} 2\right){ }^{3} \mathrm{~F}$ $11 \% 1\left|\left({ }^{2} \mathrm{G} 2\right){ }^{3} \mathrm{H}+11 \% 1\right|\left({ }^{2} \mathrm{~F} 1\right){ }^{3} \mathrm{G}+10 \% 1 \mid\left({ }^{4} \mathrm{D}\right){ }^{5} \mathrm{~F}$ $15 \% 1\left|\left({ }^{2} \mathrm{~F} 1\right){ }^{1} \mathrm{G}+9 \% 1\right|\left({ }^{4} \mathrm{G}\right){ }^{3} \mathrm{H}+8 \% 1 \mid\left({ }^{4} \mathrm{D}\right){ }^{3} \mathrm{~F}$ $25 \% 1\left|\left({ }^{4} \mathrm{G}\right) \quad{ }^{5} \mathrm{H}+19 \% 1\right|\left({ }^{4} \mathrm{D}\right) \quad{ }^{5} \mathrm{~F}+10 \% 1 \mid\left({ }^{4} \mathrm{D}\right){ }^{5} \mathrm{D}$ $23 \% 1\left|\left({ }^{4} \mathrm{G}\right) \quad{ }^{5} \mathrm{~F}+20 \% 1\right|\left({ }^{4} \mathrm{G}\right) \quad{ }^{5} \mathrm{G}+13 \% 1 \mid\left({ }^{4} \mathrm{G}\right) \quad{ }^{3} \mathrm{~F}$ $34 \% 1\left|\left({ }^{4} \mathrm{G}\right) \quad{ }^{5} \mathrm{H}+20 \% 1\right|\left({ }^{4} \mathrm{G}\right) \quad{ }^{5} \mathrm{G}+8 \% 1 \mid\left({ }^{4} \mathrm{~F}\right) \quad{ }^{5} \mathrm{G}$ $84 \% 1\left|\left({ }^{6} \mathrm{~S}\right) \quad{ }^{7} \mathrm{P}+13 \% 1\right|\left({ }^{4} \mathrm{P}\right) \quad{ }^{5} \mathrm{D}+1 \% 1 \mid\left({ }^{4} \mathrm{D}\right) \quad{ }^{5} \mathrm{~F}$

$36 \% 1\left|\left({ }^{2} \mathrm{G} 1\right){ }^{1} \mathrm{H}+19 \% 1\right|\left({ }^{2} \mathrm{G} 1\right){ }^{3} \mathrm{H}+9 \% 2 \mid\left({ }^{5} \mathrm{D}^{6} \mathrm{D}\right){ }^{7} \mathrm{D}$ $32 \% 2\left|\left({ }^{5} \mathrm{D}^{6} \mathrm{D}\right){ }^{7} \mathrm{D}+26 \% 2\right|\left({ }^{5} \mathrm{D}^{6} \mathrm{D}\right){ }^{7} \mathrm{~F}+13 \% 1 \mid\left({ }^{2} \mathrm{G} 1\right){ }^{1} \mathrm{H}$ $57 \% 1\left|\left({ }^{2} \mathrm{G} 1\right){ }^{3} \mathrm{G}+13 \% 1\right|\left({ }^{2} \mathrm{G} 1\right){ }^{3} \mathrm{H}+8 \% 1 \mid\left({ }^{2} \mathrm{G} 2\right){ }^{3} \mathrm{G}$ $31 \% 2\left|\left({ }^{3} \mathrm{H}^{4} \mathrm{H}\right){ }^{5} \mathrm{I}+15 \% 2\right|\left({ }^{3} \mathrm{H}^{4} \mathrm{H}\right){ }^{5} \mathrm{H}+15 \% 2 \mid\left({ }^{3} \mathrm{G}^{4} \mathrm{G}\right){ }^{5} \mathrm{H}$ $43 \% 2\left|\left({ }^{5} \mathrm{D}^{6} \mathrm{D}\right){ }^{7} \mathrm{~F}+16 \% 2\right|\left({ }^{5} \mathrm{D}^{6} \mathrm{D}\right){ }^{7} \mathrm{D}+8 \% 2 \mid\left({ }^{5} \mathrm{D}^{6} \mathrm{D}\right){ }^{5} \mathrm{~F}$ $29 \% 1\left|\left({ }^{2} \mathrm{H}\right) \quad{ }^{1} \mathrm{H}+20 \% 1\right|\left({ }^{2} \mathrm{H}\right) \quad{ }^{3} \mathrm{G}+11 \% 1 \mid\left({ }^{2} \mathrm{H}\right){ }^{3} \mathrm{H}$ $22 \% 1\left|\left({ }^{2} \mathrm{H}\right) \quad{ }^{1} \mathrm{H}+19 \% 1\right|\left({ }^{2} \mathrm{G} 2\right){ }^{3} \mathrm{G}+14 \% 1 \mid\left({ }^{2} \mathrm{G} 1\right){ }^{3} \mathrm{H}$ $38 \% 1\left|\left({ }^{2} \mathrm{G} 2\right){ }^{1} \mathrm{H}+14 \% 1\right|\left({ }^{2} \mathrm{G} 1\right){ }^{3} \mathrm{G}+11 \% 1 \mid\left({ }^{4} \mathrm{~F}\right){ }^{3} \mathrm{G}$ $24 \% 1\left|\left({ }^{2} \mathrm{~F} 2\right) \quad{ }^{3} \mathrm{G}+20 \% 1\right|\left({ }^{2} \mathrm{G} 1\right){ }^{3} \mathrm{H}+19 \% 1 \mid\left({ }^{4} \mathrm{~F}\right) \quad{ }^{5} \mathrm{G}$ $44 \% 1\left|\left({ }^{2} \mathrm{~F} 1\right){ }^{3} \mathrm{G}+11 \% 1\right|\left({ }^{2} \mathrm{G} 1\right){ }^{3} \mathrm{H}+8 \% 1 \mid\left({ }^{4} \mathrm{D}\right){ }^{5} \mathrm{~F}$ $20 \% 1\left|\left({ }^{2} \mathrm{~F} 2\right) \quad{ }^{3} \mathrm{G}+20 \% 1\right|\left({ }^{2} \mathrm{I}\right) \quad{ }^{1} \mathrm{H}+12 \% 1 \mid\left({ }^{2} \mathrm{H}\right) \quad{ }^{3} \mathrm{H}$ $22 \% 1\left|\left({ }^{2} \mathrm{G} 2\right) \quad{ }^{3} \mathrm{H}+20 \% 1\right|\left({ }^{4} \mathrm{~F}\right) \quad{ }^{5} \mathrm{~F}+10 \% 1 \mid\left({ }^{2} \mathrm{G} 2\right){ }^{3} \mathrm{G}$ $18 \% 1\left|\left({ }^{2} \mathrm{I}\right) \quad{ }^{1} \mathrm{H}+14 \% 1\right|\left({ }^{4} \mathrm{~F}\right) \quad{ }^{3} \mathrm{G}+11 \% 1 \mid\left({ }^{2} \mathrm{G} 2\right){ }^{3} \mathrm{H}$ $27 \% 1\left|\left({ }^{2} \mathrm{H}\right) \quad{ }^{3} \mathrm{H}+24 \% 1\right|\left({ }^{2} \mathrm{I}\right) \quad{ }^{3} \mathrm{H}+18 \% 1 \mid\left({ }^{2} \mathrm{H}\right) \quad{ }^{3} \mathrm{G}$ $22 \% 1\left|\left({ }^{2} \mathrm{H}\right) \quad{ }^{3} \mathrm{I} \quad+22 \% 1\right|\left({ }^{2} \mathrm{G} 2\right){ }^{3} \mathrm{G}+10 \% 1 \mid\left({ }^{2} \mathrm{I}\right) \quad{ }^{3} \mathrm{H}$ $14 \% 1\left|\left({ }^{4} \mathrm{~F}\right) \quad{ }^{5} \mathrm{~F}+14 \% 1\right|\left({ }^{2} \mathrm{H}\right) \quad{ }^{3} \mathrm{G}+13 \% 1 \mid\left({ }^{2} \mathrm{I}\right) \quad{ }^{3} \mathrm{I}$ $25 \% 1\left|\left({ }^{2} \mathrm{I}\right) \quad{ }^{3} \mathrm{I} \quad+18 \% 1\right|\left({ }^{2} \mathrm{I}\right) \quad{ }^{1} \mathrm{H}+15 \% 1 \mid\left({ }^{2} \mathrm{G} 2\right){ }^{1} \mathrm{H}$ $13 \% 1\left|\left({ }^{2} \mathrm{~F} 2\right) \quad{ }^{3} \mathrm{G}+12 \% 1\right|\left({ }^{4} \mathrm{~F}\right) \quad{ }^{5} \mathrm{G}+11 \% 1 \mid\left({ }^{2} \mathrm{I}\right) \quad{ }^{3} \mathrm{H}$ $47 \% 1\left|\left({ }^{4} \mathrm{G}\right) \quad{ }^{3} \mathrm{G}+11 \% 1\right|\left({ }^{4} \mathrm{D}\right) \quad{ }^{5} \mathrm{~F}+8 \% 1 \mid\left({ }^{4} \mathrm{G}\right){ }^{5} \mathrm{H}$ $28 \% 1\left|\left({ }^{2} \mathrm{H}\right) \quad{ }^{3} \mathrm{I}+15 \% 1\right|\left({ }^{4} \mathrm{~F}\right) \quad{ }^{3} \mathrm{G}+11 \% 1 \mid\left({ }^{4} \mathrm{D}\right){ }^{5} \mathrm{~F}$ $32 \% 1\left|\left({ }^{4} \mathrm{D}\right) \quad{ }^{5} \mathrm{~F}+27 \% 1\right|\left({ }^{4} \mathrm{G}\right) \quad{ }^{3} \mathrm{H}+8 \% 1 \mid\left({ }^{4} \mathrm{G}\right) \quad{ }^{3} \mathrm{G}$ 


\begin{tabular}{|c|c|c|c|c|c|c|c|c|c|c|c|c|c|}
\hline 139739.81 & 139761.77 & -21.96 & 16 & 15 & 0.08 & 0.06 & 0.10 & $18 \% 1 \mid\left({ }^{4} \mathrm{G}\right)$ & ${ }^{5} \mathrm{~F}$ & $+17 \% 1 \mid\left({ }^{4} \mathrm{G}\right)$ & ${ }^{5} \mathrm{G}$ & $+13 \% 1 \mid\left({ }^{2} \mathrm{G} 2\right)$ & ${ }^{3} \mathrm{H}$ \\
\hline 137685.88 & 137697.62 & -11.74 & 11 & 9 & 0.10 & 0.07 & 0.12 & $35 \% 1 \mid\left({ }^{4} \mathrm{G}\right)$ & ${ }^{3} \mathrm{H}$ & $+25 \% 1 \mid\left({ }^{4} \mathrm{D}\right)$ & ${ }^{5} \mathrm{~F}$ & $+11 \% 1 \mid\left({ }^{4} F\right)$ & ${ }^{5} \mathrm{G}$ \\
\hline 136429.29 & 136406.26 & 23.03 & 12 & 10 & 0.11 & 0.05 & 0.13 & $34 \% 1 \mid\left({ }^{4} \mathrm{G}\right)$ & ${ }^{5} \mathrm{H}$ & $+27 \% 1 \mid\left({ }^{4} \mathrm{G}\right)$ & ${ }^{5} \mathrm{G}$ & $+9 \% 1\left({ }^{4} \mathrm{~F}\right)$ & ${ }^{5} \mathrm{~F}$ \\
\hline 133412.78 & 133429.12 & -16.34 & 8 & 7 & 0.09 & 0.07 & 0.12 & $20 \% 1 \mid\left({ }^{2} \mathrm{I}\right)$ & ${ }^{3} \mathrm{I}$ & $+14 \% 1 \mid\left({ }^{2} \mathrm{I}\right)$ & ${ }^{1} \mathrm{H}$ & $+10 \% 1 \mid\left({ }^{4} G\right)$ & ${ }^{5} \mathrm{~F}$ \\
\hline 125982.14 & 125979.09 & 3.05 & 11 & 8 & 0.09 & 0.10 & 0.11 & $40 \% 1 \mid\left({ }^{4} \mathrm{G}\right)$ & ${ }^{5} \mathrm{~F}$ & $+13 \% 1 \mid\left({ }^{4} \mathrm{G}\right)$ & ${ }^{3} \mathrm{G}$ & $+13 \% 1 \mid\left({ }^{4} \mathrm{G}\right)$ & ${ }^{5} \mathrm{H}$ \\
\hline 122338.65 & 122347.35 & -8.70 & 7 & 6 & 0.12 & 0.08 & 0.14 & $32 \% 1 \mid\left({ }^{4} \mathrm{G}\right)$ & ${ }^{5} \mathrm{G}$ & $+25 \% 1 \mid\left({ }^{4} \mathrm{G}\right)$ & ${ }^{5} \mathrm{H}$ & $+11 \% 1 \mid\left({ }^{4} \mathrm{G}\right)$ & ${ }^{3} \mathrm{H}$ \\
\hline \multicolumn{14}{|c|}{ 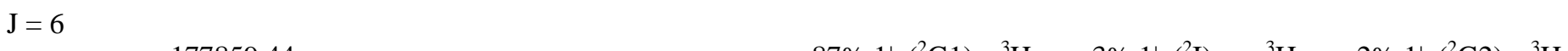 } \\
\hline - & 177859.44 & - & & & & & & $87 \% 1 \mid\left({ }^{2} \mathrm{G} 1\right)$ & ${ }^{3} \mathrm{H}$ & $+3 \% 1 \mid\left({ }^{2} \mathrm{I}\right)$ & ${ }^{3} \mathrm{H}$ & $+2 \% 1 \mid\left({ }^{2} \mathrm{G} 2\right)$ & ${ }^{3} \mathrm{H}$ \\
\hline 169715.71 & 169718.84 & -3.13 & 7 & 7 & 0.16 & 0.16 & 0.30 & $26 \% 1 \mid\left({ }^{2} \mathrm{H}\right)$ & ${ }^{1} \mathrm{I}$ & $+24 \% 1 \mid\left({ }^{2} \mathrm{H}\right)$ & ${ }^{3} \mathrm{H}$ & $+21 \% 1 \mid\left({ }^{2} \mathrm{G} 2\right)$ & ${ }^{3} \mathrm{H}$ \\
\hline 165923.23 & 165917.63 & 5.60 & 4 & 4 & 0.26 & 0.15 & 0.30 & $28 \% 1 \mid\left({ }^{2} \mathrm{H}\right)$ & ${ }^{3} \mathrm{H}$ & $+25 \% 1 \mid\left({ }^{2} \mathrm{H}\right)$ & ${ }^{3} \mathrm{I}$ & $+17 \% 1 \mid\left({ }^{2} \mathrm{G} 2\right)$ & ${ }^{3} \mathrm{H}$ \\
\hline 159416.27 & 159444.71 & -28.44 & 4 & 4 & 0.18 & 0.02 & 0.21 & $37 \% 1 \mid\left({ }^{2} \mathrm{H}\right)$ & ${ }^{1} \mathrm{I}$ & $+35 \% 1 \mid\left({ }^{4} \mathrm{~F}\right)$ & ${ }^{5} \mathrm{G}$ & $+10 \% 1 \mid\left({ }^{2} \mathrm{G} 2\right)$ & ${ }^{3} \mathrm{H}$ \\
\hline 157327.79 & 157355.17 & -27.38 & 11 & 7 & 0.15 & 0.13 & 0.17 & $52 \% 1 \mid\left({ }^{2} \mathrm{I}\right)$ & ${ }^{1} \mathrm{I}$ & $+21 \% 1 \mid\left({ }^{2} \mathrm{H}\right)$ & ${ }^{3} \mathrm{I}$ & $+15 \% 1 \mid\left({ }^{2} \mathrm{I}\right)$ & ${ }^{3} \mathrm{H}$ \\
\hline 154293.71 & 154297.76 & -4.05 & 7 & 7 & 0.11 & 0.06 & 0.14 & $19 \% 1 \mid\left({ }^{2} \mathrm{I}\right)$ & ${ }^{3} \mathbf{I}$ & $+17 \% 1 \mid\left({ }^{2} \mathrm{H}\right)$ & ${ }^{1} \mathrm{I}$ & $+16 \% 1 \mid\left({ }^{2} \mathrm{H}\right)$ & ${ }^{3} \mathrm{I}$ \\
\hline 151364.78 & 151398.33 & -33.56 & 9 & 7 & 0.13 & 0.13 & 0.16 & $37 \%$ 1| ( $\left.{ }^{2} \mathrm{G} 2\right)$ & ${ }^{3} \mathrm{H}$ & $+28 \% 1 \mid\left({ }^{4} \mathrm{~F}\right)$ & ${ }^{5} \mathrm{G}$ & $+12 \% 1 \mid\left({ }^{2} \mathrm{I}\right)$ & ${ }^{3} I$ \\
\hline 149313.18 & 149324.43 & -11.25 & 9 & 9 & 0.11 & 0.08 & 0.13 & $24 \%$ 1| ( $\left.{ }^{2} \mathrm{I}\right)$ & ${ }^{3} \mathrm{~K}$ & $+21 \% 1 \mid\left({ }^{2} \mathrm{I}\right)$ & ${ }^{3} \mathrm{I}$ & $+21 \% 1\left[\left({ }^{2} \mathrm{H}\right)\right.$ & ${ }^{3} \mathrm{H}$ \\
\hline 142662.22 & 142699.80 & -37.58 & 10 & 10 & 0.12 & 0.10 & 0.15 & $31 \% 1 \mid\left({ }^{4} \mathrm{G}\right)$ & ${ }^{3} \mathrm{H}$ & $+30 \% 1 \mid\left({ }^{2} \mathrm{I}\right)$ & ${ }^{3} \mathrm{H}$ & $+12 \% 1 \mid\left({ }^{2} \mathrm{I}\right)$ & ${ }^{1} \mathrm{I}$ \\
\hline 139094.74 & 139094.77 & -0.03 & 5 & 5 & 0.15 & 0.11 & 0.18 & $59 \% 1 \mid\left({ }^{4} \mathrm{G}\right)$ & ${ }^{5} \mathrm{H}$ & $+14 \% 1 \mid\left({ }^{4} \mathrm{G}\right)$ & ${ }^{5} \mathrm{G}$ & $+7 \% 1 \mid\left({ }^{2} \mathrm{I}\right)$ & ${ }^{3} \mathrm{H}$ \\
\hline 138121.21 & 138080.45 & 40.76 & 10 & 10 & 0.09 & 0.10 & 0.15 & $30 \% 1 \mid\left({ }^{4} \mathrm{G}\right)$ & ${ }^{5} \mathrm{G}$ & $+21 \% 1 \mid\left({ }^{4} \mathrm{G}\right)$ & ${ }^{3} \mathrm{H}$ & $+20 \% 1 \mid\left({ }^{2} \mathrm{I}\right)$ & ${ }^{3} \mathrm{H}$ \\
\hline 133911.73 & 133903.23 & 8.50 & 8 & 8 & 0.08 & 0.07 & 0.11 & $53 \% 1 \mid\left({ }^{2} \mathrm{I}\right)$ & ${ }_{5}^{3} \mathrm{~K}$ & $+12 \% 1 \mid\left({ }^{4} G\right)$ & ${ }^{5} \mathrm{G}$ & $+12 \% 1 \mid\left({ }^{2} \mathrm{I}\right)$ & ${ }_{5}^{3} \mathrm{I}$ \\
\hline 122611.21 & 122660.05 & -48.84 & 6 & 3 & 0.12 & 0.10 & 0.14 & $27 \% 1 \mid\left({ }^{4} \mathrm{G}\right)$ & ${ }^{5} \mathrm{G}$ & $+27 \% 1 \mid\left({ }^{4} \mathrm{G}\right)$ & ${ }^{3} \mathrm{H}$ & $+20 \% 1 \mid\left({ }^{4} \mathrm{G}\right)$ & ${ }^{5} \mathrm{H}$ \\
\hline \multicolumn{14}{|l|}{$\mathrm{J}=7$} \\
\hline 167498.11 & 167461.81 & 36.30 & 3 & 3 & 0.26 & 0.12 & 0.30 & $69 \% 1 \mid\left({ }^{2} \mathrm{H}\right)$ & ${ }^{3} \mathrm{I}$ & $+13 \% 1 \mid\left({ }^{2} \mathrm{I}\right)$ & ${ }^{1} \mathrm{~K}$ & $+10 \% 1 \mid\left({ }^{2} \mathrm{I}\right)$ & ${ }^{5} \mathrm{~K}$ \\
\hline 153192.39 & 153160.92 & 31.47 & 6 & 5 & 0.20 & 0.06 & 0.21 & $70 \% 1 \mid\left({ }^{2} \mathrm{I}\right)$ & ${ }^{3} \mathrm{I}$ & $+15 \% 1 \mid\left({ }^{2} \mathrm{I}\right)$ & ${ }^{3} \mathrm{~K}$ & $+14 \% 1 \mid\left({ }^{2} \mathrm{I}\right)$ & $\mathrm{K}$ \\
\hline 149661.90 & 149643.60 & 18.30 & 5 & 5 & 0.20 & 0.22 & 0.30 & $43 \% 1 \mid\left({ }^{2} \mathrm{I}\right)$ & ${ }^{1} \mathrm{~K}$ & $+29 \% 1 \mid\left({ }^{2} \mathrm{I}\right)$ & ${ }^{3} \mathrm{~K}$ & $+14 \% 1 \mid\left({ }^{2} \mathrm{H}\right)$ & ${ }^{3} \mathrm{I}$ \\
\hline 138463.86 & 138420.16 & 43.70 & 5 & 5 & 0.12 & 0.14 & 0.17 & $35 \% 1 \mid\left({ }^{4} \mathrm{G}\right)$ & ${ }^{5} \mathrm{H}$ & $+25 \% 1 \mid\left({ }^{2} \mathrm{I}\right)$ & ${ }^{1} \mathrm{~K}$ & $+18 \% 1 \mid\left({ }^{2} \mathrm{I}\right)$ & ${ }^{3} \mathrm{~K}$ \\
\hline 137224.74 & 137272.36 & -47.62 & 5 & 4 & 0.13 & 0.19 & 0.16 & $45 \% 1 \mid\left({ }^{4} \mathrm{G}\right)$ & ${ }^{5} \mathrm{H}$ & $+27 \% 1 \mid\left({ }^{2} \mathrm{I}\right)$ & ${ }^{3} \mathrm{~K}$ & $+12 \% 1 \mid\left({ }^{2} \mathrm{I}\right)$ & ${ }^{3} \mathrm{I}$ \\
\hline \multicolumn{14}{|l|}{$\mathrm{J}=8$} \\
\hline 152556.11 & 152542.70 & 13.42 & 1 & 1 & 0.42 & 0.42 & 0.40 & $100 \% 1 \mid\left({ }^{2} \mathrm{I}\right)$ & ${ }^{3} \mathrm{~K}$ & & & & \\
\hline
\end{tabular}


Table 4

Fitted and calculated parameter values $\left(\mathrm{cm}^{-1}\right)$ in the $5 \mathrm{~d}^{6}, 5 \mathrm{~d}^{5} 6 \mathrm{~s}$ and $5 \mathrm{~d}^{4} 6 \mathrm{~s}^{2}$ configurations of Ir IV.

\begin{tabular}{|c|c|c|c|c|}
\hline Parameter & Fitted value & & HFR & Fitted/HFR \\
\hline \multicolumn{5}{|l|}{$5 d^{6}$} \\
\hline $\mathrm{E}_{\mathrm{av}}$ & 30705.4 & $(4.4)$ & 35693.6 & \\
\hline $\mathrm{O}_{2}$ & 6285.9 & $(5.0)$ & 7601.1 & 0.8270 \\
\hline $\mathrm{O}_{2}^{\prime}$ & 3949.4 & $(8.2)$ & 5181.0 & 0.7623 \\
\hline $\mathrm{E}_{\alpha}$ & 77.9 & $(4.1)$ & & \\
\hline $\mathrm{E}_{\beta}$ & 24.0 & $(5.0)$ & & \\
\hline$\zeta_{\mathrm{d}}$ & 4306.9 & $(3.8)$ & 4498.4 & 0.9574 \\
\hline $\mathrm{T}_{1}$ & 0.32 & $(0.16)$ & & \\
\hline $\mathrm{T}_{2}$ & 0.35 & $(0.17)$ & & \\
\hline $\mathrm{T}_{3}$ & 0.06 & $(0.16)$ & & \\
\hline $\mathrm{T}_{4}$ & 0.05 & $(0.15)$ & & \\
\hline$A_{c}$ & 30.2 & $(3.2)$ & & \\
\hline $\mathrm{A}_{3}$ & 7.0 & $(2.8)$ & & \\
\hline $\mathrm{A}_{4}$ & 5.6 & $(4.7)$ & & \\
\hline $\mathrm{A}_{5}$ & 10.2 & $(4.4)$ & & \\
\hline $\mathrm{A}_{6}$ & 19.4 & $(2.6)$ & & \\
\hline $\mathrm{A}_{1}$ & -4.0 & & & \\
\hline $\mathrm{A}_{2}$ & 4.8 & & & \\
\hline $\mathrm{A}_{0}$ & -4.0 & & & \\
\hline \multicolumn{5}{|l|}{$5 d^{5} 6 s$} \\
\hline $\mathrm{E}_{\mathrm{av}}$ & 82298.7 & $(6.0)$ & 89683.9 & $0.9556^{\mathrm{a}}$ \\
\hline $\mathrm{O}_{2}$ & 6469.4 & $(5.3)$ & 7806.2 & 0.8287 \\
\hline $\mathrm{O}_{2}^{\prime}$ & 4069.2 & $(7.6)$ & 5304.7 & 0.7671 \\
\hline $\mathrm{E}_{\alpha}$ & 90.5 & $(4.5)$ & & \\
\hline $\mathrm{E}_{\beta}$ & 40.5 & $(5.8)$ & & \\
\hline$\zeta_{\mathrm{d}}$ & 4561.7 & $(4.1)$ & 4734.3 & 0.9635 \\
\hline $\mathrm{T}_{1}$ & -0.15 & $(0.20)$ & & \\
\hline $\mathrm{T}_{2}$ & 0.0 & & & \\
\hline $\mathrm{T}_{3}$ & 0.0 & & & \\
\hline $\mathrm{T}_{4}$ & 0.0 & & & \\
\hline $\mathrm{A}_{\mathrm{c}}$ & 29.8 & $(3.4)$ & & \\
\hline $\mathrm{A}_{3}$ & 3.2 & & & \\
\hline $\mathrm{A}_{4}$ & 6.5 & & & \\
\hline $\mathrm{A}_{5}$ & 6.3 & $(3.4)$ & & \\
\hline $\mathrm{A}_{6}$ & 9.0 & $(2.1)$ & & \\
\hline $\mathrm{A}_{1}$ & -4.0 & & & \\
\hline $\mathrm{A}_{2}$ & 4.8 & & & \\
\hline $\mathrm{A}_{0}$ & -4.0 & & & \\
\hline $\mathrm{C}_{\mathrm{ds}}$ & 3027.1 & $(3.6)$ & 3756.1 & 0.8059 \\
\hline $\mathrm{T}_{\mathrm{dds}}$ & -9.2 & $(7.4)$ & & \\
\hline $\mathrm{A}_{\mathrm{mso}}$ & 55.8 & $(3.1)$ & & \\
\hline \multicolumn{5}{|l|}{$5 d^{4} 6 s^{2}$} \\
\hline $\mathrm{E}_{\mathrm{av}}$ & 149222.0 & & 160775.1 & $0.9475^{\mathrm{a}}$ \\
\hline $\mathrm{O}_{2}$ & 6650.0 & & 8001.9 & 0.8311 \\
\hline $\mathrm{O}_{2}^{\prime}$ & 4143.0 & & 5422.5 & 0.7640 \\
\hline $\mathrm{E}_{\alpha}$ & 80.0 & & & \\
\hline $\mathrm{E}_{\beta}$ & 54.0 & & & \\
\hline$\zeta_{\mathrm{d}}$ & 4793.0 & & 4972.7 & 0.9639 \\
\hline
\end{tabular}


$\begin{array}{ll}\mathrm{A}_{\mathrm{c}} & 37.2 \\ \mathrm{~A} & \end{array}$

$\begin{array}{ll}\mathrm{A}_{3} & 6.7\end{array}$

$\begin{array}{ll}\mathrm{A}_{4} & 9.4\end{array}$

$\begin{array}{ll}\mathrm{A}_{5} & 10.5\end{array}$

$\mathrm{A}_{6} \quad 15.5$

$\mathrm{A}_{1}-4.4$

$\mathrm{A}_{2} \quad 5.1$

$\mathrm{A}_{0} \quad-4.4$

$\mathrm{R}^{2}(\mathrm{dd}, \mathrm{ds}) 12^{\mathrm{b}} \quad-22308.0$

$\mathrm{R}^{2}$ (dd, ss) $12 \quad 20073.0$

$\mathrm{R}^{2}(\mathrm{dd}, \mathrm{ds}) 23 \quad-22302.0$ $\begin{array}{ll}-26636.3 & 0.8375\end{array}$

$23942.8 \quad 0.8384$

$-26629.3 \quad 0.8375$

Mean Deviation $^{\mathbf{c}}=20 \mathrm{~cm}^{-1}$

a The energy differences with respect to the ground configuration were used: $\left(\mathrm{E}_{\mathrm{av}}(\mathrm{conf})-\mathrm{E}_{\mathrm{av}}\left(5 \mathrm{~d}^{6}\right)\right)_{\mathrm{LSF}} /\left(\mathrm{E}_{\mathrm{av}}(\mathrm{conf})-\mathrm{E}_{\mathrm{av}}\left(5 \mathrm{~d}^{6}\right)\right)_{\mathrm{HFR}}$, where "conf" is either $5 \mathrm{~d}^{5} 6 \mathrm{~s}$ or $5 \mathrm{~d}^{4} 6 \mathrm{~s}^{2}$.

b "xy" in the end of the $R$-parameter names means interaction between configurations $\mathrm{x}$ and $\mathrm{y}$, where 1 stands for $5 \mathrm{~d}^{6}, 2$ stands for $5 \mathrm{~d}^{5} 6 \mathrm{~s}$ and 3 stands for $5 \mathrm{~d}^{4} 6 \mathrm{~s}^{2}$.

c Mean Deviation $=\left[\left(\Sigma\left(\mathrm{E}_{\text {obs }}-\mathrm{E}_{\text {calc }}\right)^{2}\right) /(\mathrm{n}-\mathrm{m})\right]^{1 / 2}$, where $\mathrm{n}$ is the number of known levels, $\mathrm{m}$ is the number of free parameters. 
Table 5

Fitted and calculated parameter values $\left(\mathrm{cm}^{-1}\right)$ in the $5 d^{5} 6 p, 5 d^{4} 6 s 6 p$ and $5 d^{3} 6 s^{2} 6 p$ configurations of Ir IV.

\begin{tabular}{|c|c|c|c|c|}
\hline Parameter & Fitted value & & HFR & Fitted/HFR \\
\hline \multicolumn{5}{|l|}{$5 d^{5} 6 p$} \\
\hline $\mathrm{E}_{\mathrm{av}}$ & 154947.3 & $(5.9)$ & 159905.7 & $1.0002^{\mathrm{a}}$ \\
\hline $\mathrm{O}_{2}$ & 6514.7 & $(6.8)$ & 7864.5 & 0.8284 \\
\hline $\mathrm{O}_{2}^{\prime}$ & 4072.1 & $(8.8)$ & 5339.5 & 0.7626 \\
\hline $\mathrm{E}_{\alpha}$ & 96.8 & $(5.9)$ & & \\
\hline $\mathrm{E}_{\beta}$ & 67.7 & $(7.7)$ & & \\
\hline$\zeta_{\mathrm{d}}$ & 4665.4 & $(4.7)$ & 4794.1 & 0.9732 \\
\hline $\mathrm{T}_{1}$ & 0.83 & $(0.28)$ & & \\
\hline $\mathrm{T}_{2}$ & 0.47 & $(0.55)$ & & \\
\hline $\mathrm{T}_{3}$ & -0.63 & $(0.48)$ & & \\
\hline $\mathrm{T}_{4}$ & 0.18 & $(0.31)$ & & \\
\hline $\mathrm{A}_{\mathrm{c}}$ & 40.1 & $(5.8)$ & & \\
\hline $\mathrm{A}_{3}$ & 3.5 & & & \\
\hline $\mathrm{A}_{4}$ & 2.4 & $(5.8)$ & & \\
\hline $\mathrm{A}_{5}$ & 3.8 & $(5.3)$ & & \\
\hline $\mathrm{A}_{6}$ & 15.3 & $(3.2)$ & & \\
\hline $\mathrm{A}_{1}$ & -3.0 & & & \\
\hline $\mathrm{A}_{2}$ & 3.0 & & & \\
\hline $\mathrm{A}_{0}$ & -3.0 & & & \\
\hline $\mathrm{C}_{1}(\mathrm{dp})$ & 2558.2 & $(9.3)$ & 3052.0 & 0.8382 \\
\hline $\mathrm{C}_{2}(\mathrm{dp})$ & 2184.2 & $(7.5)$ & 2898.4 & 0.7536 \\
\hline $\mathrm{C}_{3}(\mathrm{dp})$ & 1187.3 & (9.1) & 1531.1 & 0.7754 \\
\hline $\mathrm{S}_{1}(\mathrm{dp})$ & 153.1 & (10.0) & & \\
\hline $\mathrm{S}_{2}(\mathrm{dp})$ & -92.5 & $(6.8)$ & & \\
\hline$\zeta_{\mathrm{p}}$ & 10047.4 & (13.2) & 8907.4 & 1.1280 \\
\hline $\mathrm{S}_{\mathrm{d}} \cdot \mathrm{L}_{\mathrm{p}}$ & -90.5 & (7.4) & & \\
\hline $\mathrm{S}_{\mathrm{p}} \cdot \mathrm{L}_{\mathrm{d}}$ & -28.2 & (10.9) & & \\
\hline $\mathrm{Z}_{\mathrm{pp}}^{2}$ & -36.6 & (11.1) & & \\
\hline $\mathrm{Z}_{\mathrm{dd}^{\prime}}^{2^{\mathrm{pp}}}$ & 64.1 & (9.1) & & \\
\hline $\mathrm{Z}_{\mathrm{pp}}^{1}$ & 141.6 & $(7.4)$ & & \\
\hline $\mathrm{Z}_{\mathrm{dd}^{\prime}}^{\mathrm{pp}}$ & -23.6 & (7.3) & & \\
\hline $\mathrm{Z}_{\mathrm{pp}}^{3}$ & 66.9 & $(7.7)$ & & \\
\hline $\mathrm{Z}_{\mathrm{dd}^{\prime}}^{3^{\mathrm{pp}}}$ & -29.6 & $(7.8)$ & & \\
\hline $\mathrm{SS}_{02}$ & -15.6 & (5.7) & & \\
\hline $\mathrm{SS}_{20}$ & -24.3 & $(8.4)$ & & \\
\hline $\mathrm{T}_{16}$ & -50.4 & $(7.1)$ & & \\
\hline $\mathrm{T}_{17}$ & 7.4 & $(5.3)$ & & \\
\hline $\mathrm{T}_{18}$ & -10.7 & $(8.7)$ & & \\
\hline $\mathrm{T}_{19}$ & -7.7 & $(5.2)$ & & \\
\hline $\mathrm{T}_{20}$ & -33.9 & $(7.7)$ & & \\
\hline $\mathrm{T}_{21}$ & -11.2 & $(4.2)$ & & \\
\hline $\mathrm{T}_{22}$ & -10.2 & $(6.6)$ & & \\
\hline $\mathrm{T}_{23}$ & -15.1 & $(6.0)$ & & \\
\hline $\mathrm{T}_{24}$ & -8.1 & $(5.6)$ & & \\
\hline $\mathrm{T}_{25}$ & 10.1 & $(5.6)$ & & \\
\hline $\mathrm{T}_{26}$ & -19.7 & $(7.5)$ & & \\
\hline $\mathrm{T}_{27}$ & 1.4 & $(5.7)$ & & \\
\hline $\mathrm{T}_{28}$ & 39.1 & $(8.2)$ & & \\
\hline $\mathrm{T}_{29}$ & -24.9 & $(6.3)$ & & \\
\hline $\mathrm{T}_{30}$ & 14.5 & $(7.0)$ & & \\
\hline
\end{tabular}




$\begin{array}{lrr}\mathrm{T}_{31} & -10.2 & (7.8) \\ \mathrm{T}_{32} & 8.4 & (5.6) \\ \mathrm{T}_{33} & 12.2 & (6.9) \\ \mathrm{T}_{34} & -28.8 & (7.3) \\ \mathrm{T}_{35} & -79.2 & (6.0)\end{array}$

\begin{tabular}{|c|c|c|c|}
\hline $5 d^{4} 6 s 6 p$ & & & \\
\hline $\mathrm{E}_{\mathrm{av}}$ & 218933.0 & 225922.7 & $0.9895^{\mathrm{a}}$ \\
\hline $\mathrm{O}_{2}$ & 6676.0 & 8053.0 & 0.8290 \\
\hline $\mathrm{O}_{2}^{\prime}$ & 4177.0 & 5452.8 & 0.7660 \\
\hline $\mathrm{E}_{\alpha}$ & 75.0 & & \\
\hline$E_{\beta}$ & 63.0 & & \\
\hline$\zeta_{\mathrm{d}}$ & 4892.0 & 5028.5 & 0.9729 \\
\hline $\mathrm{A}_{\mathrm{c}}$ & 23.0 & & \\
\hline $\mathrm{A}_{3}$ & 3.5 & & \\
\hline $\mathrm{A}_{4}$ & 4.0 & & \\
\hline $\mathrm{A}_{5}$ & 4.5 & & \\
\hline $\mathrm{A}_{6}$ & 12.5 & & \\
\hline $\mathrm{A}_{1}$ & -3.0 & & \\
\hline $\mathrm{A}_{2}$ & 3.0 & & \\
\hline $\mathrm{A}_{0}$ & -3.0 & & \\
\hline $\mathrm{C}_{\mathrm{ds}}$ & 3062.0 & 3749.3 & 0.8167 \\
\hline $\mathrm{T}_{\mathrm{dds}}$ & 6.0 & & \\
\hline $\mathrm{A}_{\mathrm{mso}}$ & 53.0 & & \\
\hline $\mathrm{A}_{\mathrm{ss}}$ & 0.0 & & \\
\hline $\mathrm{C}_{1}(\mathrm{dp})$ & 2747.0 & 3229.3 & 0.8507 \\
\hline $\mathrm{C}_{2}(\mathrm{dp})$ & 2268.0 & 2976.9 & 0.7619 \\
\hline $\mathrm{C}_{3}(\mathrm{dp})$ & 1205.0 & 1552.1 & 0.7764 \\
\hline$S_{1}(d p)$ & 149.0 & & \\
\hline$S_{2}(d p)$ & -94.0 & & \\
\hline$\zeta_{\mathrm{p}}$ & 11301.0 & 10022.9 & 1.1275 \\
\hline$S_{d} \cdot L_{p}$ & -82.7 & & \\
\hline$S_{p} \cdot L_{d}$ & -8.1 & & \\
\hline $\mathrm{Z}^{2} \mathrm{pp}^{\prime}$ & -52.7 & & \\
\hline$Z_{\mathrm{dd}^{\prime}}^{2^{\mathrm{t}}}$ & 59.2 & & \\
\hline $\mathrm{Z}_{, \mathrm{pp}^{\prime}}^{1}$ & 142.9 & & \\
\hline $\mathrm{Z}_{\mathrm{dd}^{\prime}}^{1^{\mathrm{pp}}}$ & -1.5 & & \\
\hline $\mathrm{Z}_{\mathrm{pp} \mathrm{p}^{\prime}}^{3}$ & 45.2 & & \\
\hline $\mathrm{Z}_{\mathrm{dd}^{\prime}}^{\mathrm{pp}}$ & -12.3 & & \\
\hline $\mathrm{C}_{\mathrm{sp}}$ & 11652.0 & 14039.6 & 0.8299 \\
\hline $\mathrm{A}_{\mathrm{mso}}(\mathrm{sp})$ & -500.0 & & \\
\hline $5 d^{3} 6 s^{2} 6 p$ & & & \\
\hline$E_{a v}$ & 297503.0 & 309287.7 & $0.9752^{\mathrm{a}}$ \\
\hline $\mathrm{O}_{2}$ & 6824.0 & 8231.9 & 0.8290 \\
\hline $\mathrm{O}_{2}$ & 4259.0 & 5560.1 & 0.7660 \\
\hline $\mathrm{E}_{\alpha}$ & 75.0 & & \\
\hline$E_{\beta}$ & 63.0 & & \\
\hline$\zeta_{\mathrm{d}}$ & 5121.0 & 5263.7 & 0.9729 \\
\hline $\mathrm{C}_{1}(\mathrm{dp})$ & 2900.0 & 3408.2 & 0.8509 \\
\hline $\mathrm{C}_{2}(\mathrm{dp})$ & 2331.0 & 3060.0 & 0.7618 \\
\hline $\mathrm{C}_{3}(\mathrm{dp})$ & 1223.0 & 1575.5 & 0.7763 \\
\hline $\mathrm{S}_{1}(\mathrm{dp})$ & 149.0 & & \\
\hline$S_{2}(d p)$ & -94.0 & & \\
\hline$\zeta_{\mathrm{p}}$ & 12661.0 & 11229.6 & 1.1275 \\
\hline
\end{tabular}




$\begin{array}{lrrr}\mathrm{R}^{2}(\mathrm{dd}, \mathrm{ds}) 12^{\mathbf{b}} & -21037.0 & -26550.0 & 0.7924 \\ \mathrm{R}^{2}(\mathrm{dp}, \mathrm{sp}) 12 & -18440.0 & -23421.2 & 0.7873 \\ \mathrm{R}^{1}(\mathrm{dp}, \mathrm{ps}) 12 & -16563.0 & -21160.6 & 0.7827 \\ \mathrm{R}^{2}(\mathrm{dd}, \mathrm{ss}) 13 & 19801.0 & 23856.7 & 0.8300 \\ \mathrm{R}^{2}(\mathrm{dd}, \mathrm{ds}) 23 & -22050.0 & -26566.7 & 0.8300 \\ \mathrm{R}^{2}(\mathrm{dp}, \mathrm{sp}) 23 & -19847.0 & -23911.5 & 0.8300 \\ \mathrm{R}^{1}(\mathrm{dp}, \mathrm{ps}) 23 & -17831.0 & -21483.7 & 0.8300\end{array}$

Mean Deviation $^{\mathrm{c}}=36 \mathrm{~cm}^{-1}$

a The energy differences with respect to the ground configuration were used: $\left(E_{a v}(\text { conf })-E_{a v}\left(5 d^{6}\right)\right)_{L S F} /\left(E_{a v}(c o n f)-E_{a v}\left(5 d^{6}\right)\right)_{H F R}$, where "conf" is either $5 d^{5} 6 p, 5 d^{4} 6 s 6 p$ or $5 d^{3} 6 s^{2} 6 p$.

b "xy" in the end of the $R$-parameter names means interaction between configurations $\mathrm{x}$ and $\mathrm{y}$, where 1 stands for $5 d^{5} 6 p, 2$ stands for $5 d^{4} 6 s 6 p$ and 3 stands for $5 d^{3} 6 s^{2} 6 p$.

c Mean Deviation $=\left[\left(\Sigma\left(\mathrm{E}_{\mathrm{obs}}-\mathrm{E}_{\mathrm{calc}}\right)^{2}\right) /(\mathrm{n}-\mathrm{m})\right]^{1 / 2}$, where $\mathrm{n}$ is the number of known levels, $\mathrm{m}$ is the number of free parameters. 